ORNL/TM-12623

Solid State Division

\title{
Solidification Microstructures in Single-Crystal Stainless Steel Melt Pools ${ }^{\ddagger}$
}

\author{
J. B. Sipf, L. A. Boatner, and S. A. David*
}

\author{
DATE PUBLISHED- \\ March 1994
}

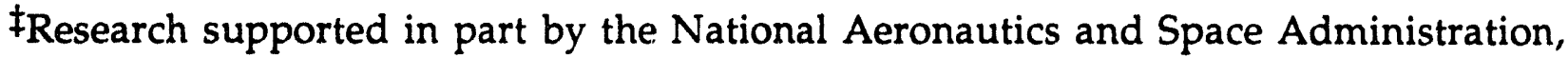
Microgravity Science and Applications Program under NASA Government Order Number: H-13059D, and by the Division of Materials Sciences, U.S. Department of Energy under Contract Number DE-AC05-84OR21400 with Martin Marietta Energy Systems, Inc.
\end{abstract}

\section{*Metals and Ceramics Division}

NOTICE: This document contains information of a preliminary nature. It is subject to revision or correction and therefore does not represent a final report.

\section{Managed by}

MARTIN MARIETTA ENERGY SYSTEMS, INC. operating the

Oak Ridge National Laboratory

for the

U.S. DEPARTMENT OF ENERGY

under contract no. DE-AC05-84OR21400 


\section{ABSTRACT}

The microstructural analysis of stationary-melt or "weld" pools provides important information concerning the relationships between the weld-pool shape, the crystallography of the sample, and the resulting microstructural properties of the weld. A detailed microstructural analysis of stationary melt pools made using oriented stainless steel single crystals with a composition of $708 \mathrm{Fe}-15 \% \mathrm{Ni}-15 \% \mathrm{Cr}$ (wt $\%$ ) was performed in order to investigate the resulting microstructure and the effects of crystallography in deterining microstructural development. Stationary melt pools were formed by employing electron-beam and gas-tungsten-arc heating methods on the (001), (011), and (111) oriented planes of the austenitic, FCC-alloy crystals. The characterization and analysis of the resulting microstructure was carried out for each crystallographic plane and welding method. The results showed that crystallography which favors "easy growth" along the <100> family of directions is a controlling factor in the microstructural formation along with the melt-pool shape. The microstructure was found to depend on the melting method, since each method forms a unique melt-pool shape. These results are used in making a three-dimensional reconstruction of the microstructure for each plane and melting method employed. This investigation also suggests avenues for future research into the 
microstructural properties of electron-beam welds as well as providing an experimental basis for mathematical models for the prediction of solidification microstructures. 
TABLE OF CONHERTIS

CENPIHR

PAGE

I. INTRODUCTION. ........................

Solidification Microstructures and

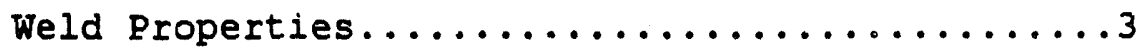

Researıh Background...................

Purpose of Investigation.................

II. IITERATURE REVIEW. ...................

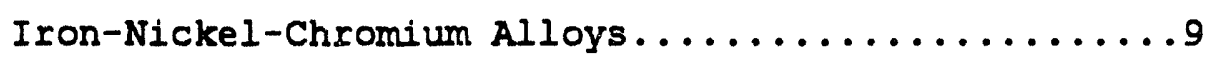

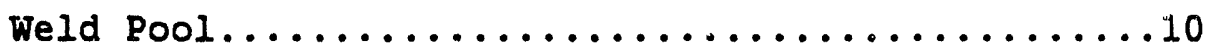

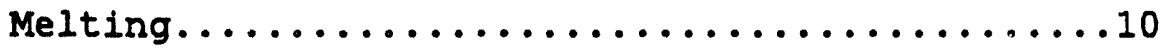

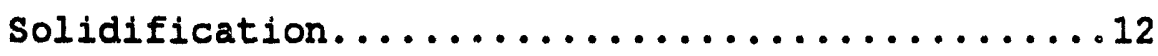

Stainless Steel single Crystal Welding..........4

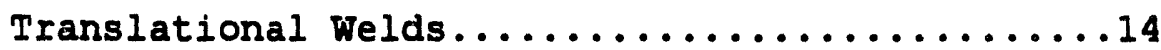

Multipass welds.....................20

Bicrystal Welds.....................22

Single Crystal welding...................24

Copper single Crystal Welds.............24

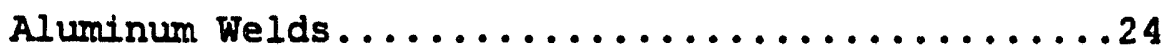

Aluminum and $\mathrm{zinc}$ Welds................ 27

Weld Ripples........................27

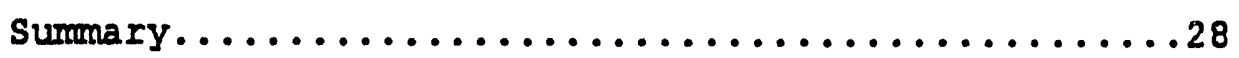




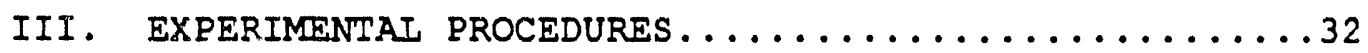

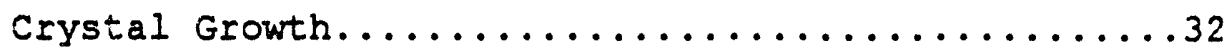

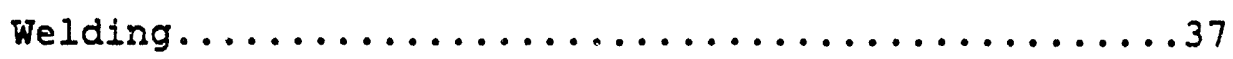

Electron Beam Welding.................... 37

Gas Tungsten Arc Welding....................

Metallographic Procedures..............42

Sectioning of the Welded Crystals..........42

Sample Mounting, Polishing, and Etching.......42

Photography $\ldots \ldots \ldots \ldots \ldots \ldots \ldots \ldots \ldots \ldots \ldots$

Microhardness......................44

Profilometry......................45

summary...........................46

IV. RESULTS AND DISCUSSION................48

Microstructural Analysis Gas Tungsten Arc Welding

$75 \%$ Helium and $25 \%$ Argon..................... 49

(001) Crystallographic orientation..........49

Sectioning Along the [100] Direction........49

Top surface.......................58

Sectioning Along the [110] Direction.......65

Summary of (001) Orientation.............73

(011) Crystallographic orientation..........73

Sectioning Along the [100] Direction........75

Top surface....................... 81

Sectioning Along the [110] Direction........90 
Sectioning Along the [111] Direction........95

Summary of (011) Orientation..............100

(111) Crystallographic orientation...........100

Sectioning Along the [110] Direction........102

Top surface........................102

summary of (111) orientation...................

Gas Tungsten Arc Rotational Welds............107

(001) Crystallographic Orientation............108

(011) Crystallographic Orientation...........123

(111) Crystallographic Orientation...........131

Comparison of Gas Tungsten Arc Rotational to

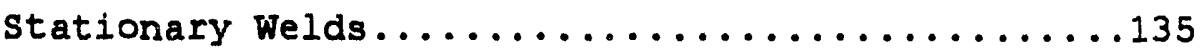

Comparison of (001) and (011) Profilometry.......139

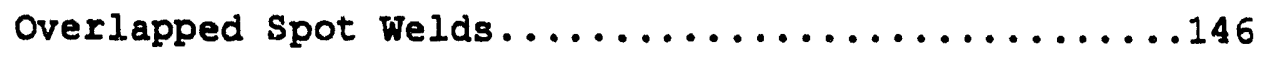

Comparison Between Gas Tungsten Arc 1008 Argon and

GTA 758 Helium and $25 \%$ Argon..................

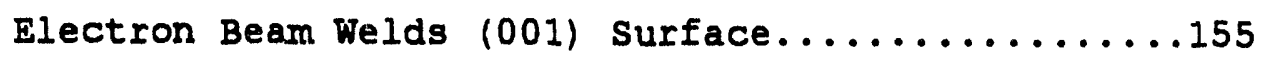

(001) Crystallographic Orientation...........155

Sectioned for Transverse Views..................

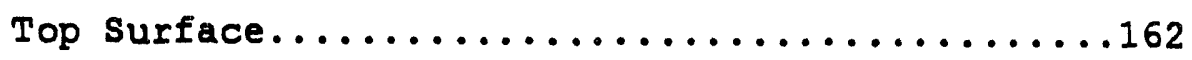

[110] Transverse Views...................171

[100] Transverse Views..................171

Summary EB (001) Crystallographic orientation....174 
Comparison Between Electron Beam and Gas Tungsten

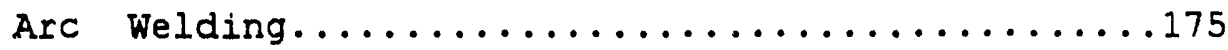

summary..............................

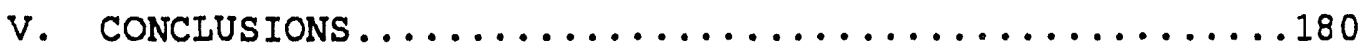

Future Work............................

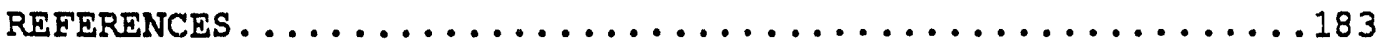

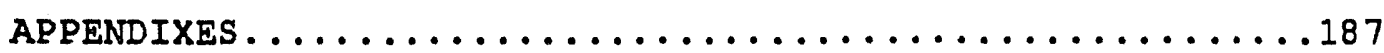

Appendix A Initial Melting Studies.................

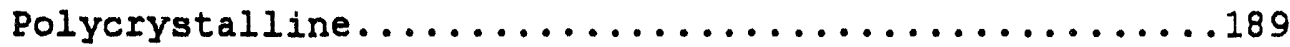

Welding Parameters..........................

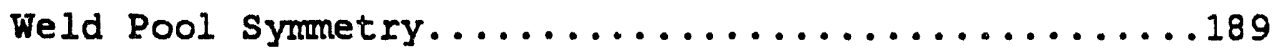

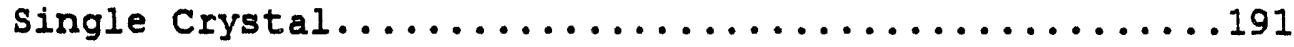

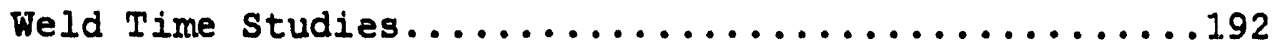

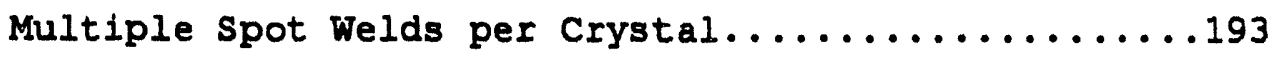

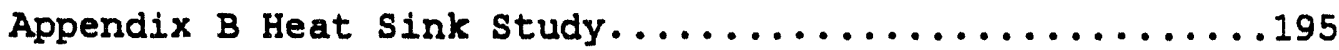

Appendix C Tri-ArC Melting Results..............199

Appendix D Microhardness study................204

Appendix E Chemical Analysis Report.............210

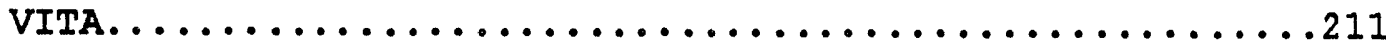


1. Impurity Elemental Analysis of Stainless steel

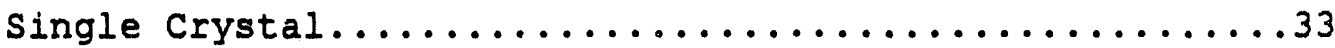

2. Electron Beam welding Parameters..........................

3. Gas Tungsten Arc Welding Parameters ...............41

4. Variations in penetration with Increased

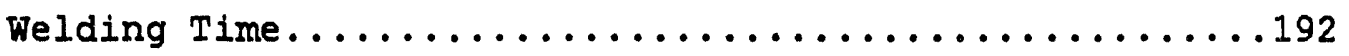

5. Tri-Arc Welding Parameters......................200 


\section{IIST OF FIGURES}

FIGURE

PAGS

1. Schematic for Spot Welding Configuration............4

2. Stainless Steel Phase Diagram....................

3. Schematic for Translational Electron Beam welds........15

4. 3-Dimensional Model for the Translational Electron

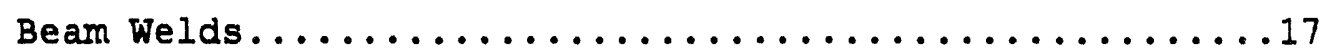

5. Welding (001) Surface Along [110] Direction with Translational speed of (a) $42 \mathrm{~mm} / \mathrm{s}$ (b) $4.2 \mathrm{~mm} / \mathrm{s} \ldots \ldots \ldots 18$

6. Multipass Translational Electron Beam Welds $4.2 \mathrm{~mm} / \mathrm{s}$ Along the $[110]$ direction (001) surface............21

7. Convection in a Laser Melt for a Scan Velocity (a) Zero

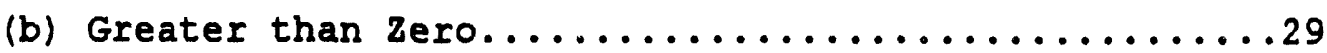

8. Crystal Boule................................

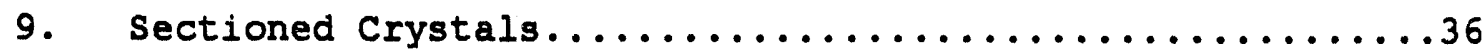

10. Electron Beam Welding system......................

11. Gas Tungsten Arc Welding system................40

12. Sectioning Configuration for Angled Cuts............43

13. Stereographic Projection for (001) Plane...............50

14. Schematic for (001) Surface [100] Direction

Sectioning and welding Configuration................ 
15. Three-Dimensional Reconstruction of (001) Plane [100]

Direction Stationary GTA Weld with Accompanying

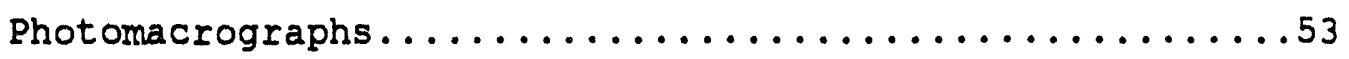

16. Three-Dimensional Reconstruction Identifying High

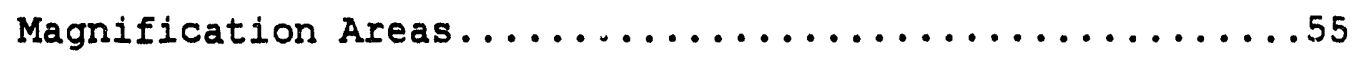

17. [100] Transverse View of Stationary GTA Weld Dendritic Growth in the Weld (Area "A" of Figure 16) .........56

18. [100] Transverse View of Stationary GTA Weld Solidification Phenomena (Area "B" of Figure 16) ......57

19. Center section of [100] Transverse View (Area "C" of

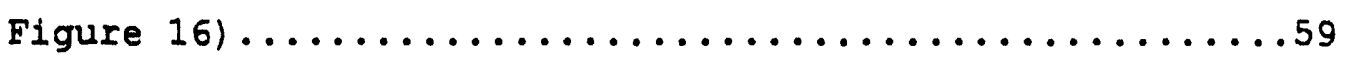

20. Schematic for (001) surface welding Configuration......60

21. Three-Dimensional Reconstruction of (001) Plane

Surface Stationary GTA Weld with Accompanying

Photomacrographs.......................61

22. Three-Dimensional Reconstruction Identifying High

Magnification Areas........................63

23. Lower Quadrant of (001) Surface of GTA Stationary

Weld High Magnification Showing Competitive

Growth (Area "A" of Figure 22) .................64

24. (001) Surface Stationary GTA Weld Center section

Symmetry (Area "B" of Figure 22) .............65

25. Schematic for (001) Surface [110] Direction

Sectioning and Welding Configuration............67 
26. Three-Dimensional Reconstruction of (001) Plane [110]

Direction Stationary GTA Weld with Accompanying

Photomacrographs.....................68

27. Three-Dimensional Reconstruction Identifying High

Magnification Areas...........................

28. Center Section of [110] Transverse View (Area "A" of

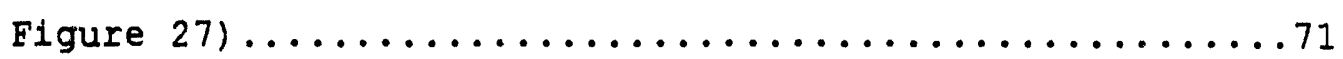

29. Center Section of [110] Transverse View (Area "B" of

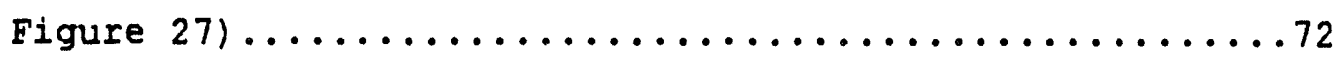

30. Stereographic Projection of the (011) Plane..........74

31. Schematic for (011) Plane [100] Direction Sectioning

and Welding Configuration...................

32. Three-Dimensional Reconstruction of (011) Plane [100]

Direction Stationary GTA Weld with Accompanying

photomacrographs........................

33. Three-Dimensional Reconstruction Identifying High

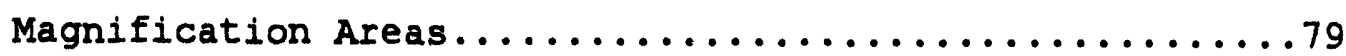

34. (011) Stationary GTA Weld [100] Transverse View for

Solidification Features (Area "A" of Figure 33) ......80

35. (011) Stationary GTA Weld [100] Transverse View for

Solidification Features (Area "B" of Figure 33) ......81

36. Schematic for the (011) Plane Weldisg Configuration.....83

37. Three-Dimensional Reconstruction of (011) Plane Surface

Stationary GTA Weld with Accompanying Photomacrographs..84 
38. Three-Dimensional Reconstruction Identifying High

Magnification Areas...................... 66

39. Center section of (011) surface Weld (Area "A" of

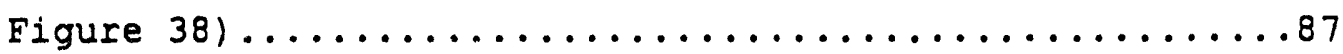

40. Left Side of Center Section (011) Surface Weld (Area

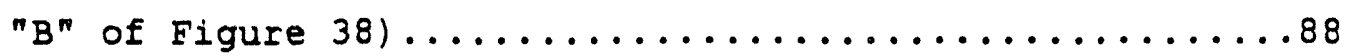

41. Right Side of Center Section (011) Surface Weld (Area

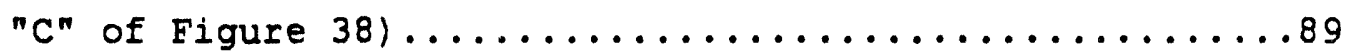

42. Schematic for (011) Surface [110] Direction sectioning

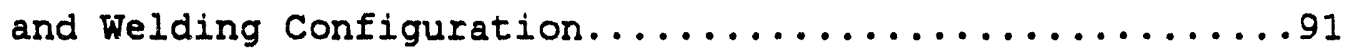

43. Three-Dimensional Reconstruction of (011) Plane [110]

Direction Stationary GTA Weld with Accompanying

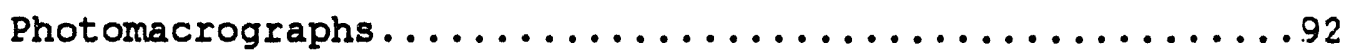

44. Three-Dimensional Reconstruction Identifying High

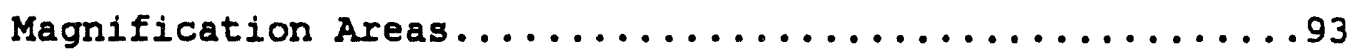

45. (011) Stationary GTA Weld [110] Transverse View

High Magnification (Area "A" of Figure 44).........94

46. Schematic for (011) Surface [111] Direction sectioning

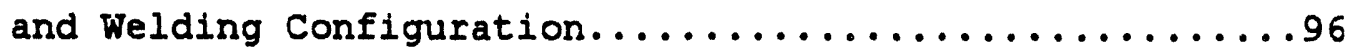

47. Three-Dimensional Reconstruction of (011) Plane [111]

Direction Stationary GTA Weld with Accompanying

Photomacrographs.

48. Three-Dimensional Reconstruction Identifying High

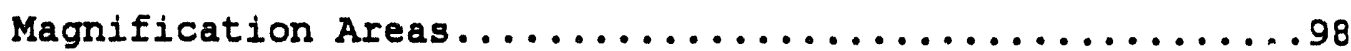


49. (011) Stationary GTA Weld [111] Transverse View High Magnification (Area "A" of Figure 48 )..............99

50. Stereographic Projection for the (111) Plane..........101

51. Schematic for (111) Surface [110] Direction Sectioning

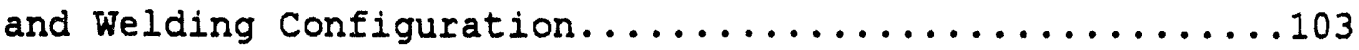

52. Three-Dimensional Reconstruction of (111) Plane [110] Direction Stationary GTA Weld with Accompanying

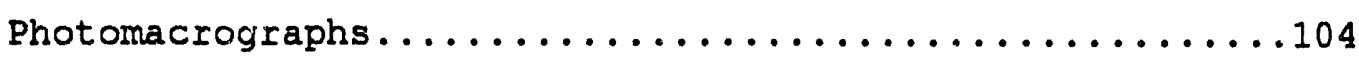

53. Schematic for (111) surface Welding Configuration.......105

54. Three-Dimensional Reconstruction of (111) Plane Surface Stationary GTA. Weld with Accompanying Photomacrographs..106

55. Three-Dimensional Reconstruction (001) Plane [100]

Direction Rotational GTA Weld with Accompanying

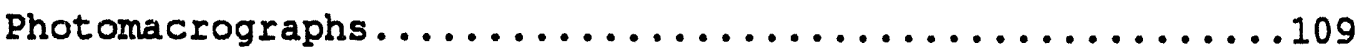

56. Three-Dimensional Reconstruction (001) Plane surface Rotational GTA Weld with Accompanying Photomacrographs..110

57. Three-Dimensional Reconstruction (001) Plane [110]

Iirection Rotational GTA Weld with Accompanying Photomacrographs...........................11

58. Three-Dimensional Reconstruction Identifying High

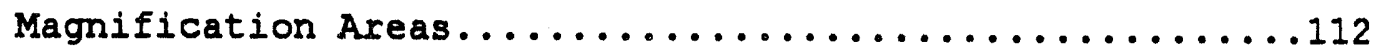

59. Transverse View GTA Rotational Weld [100] Direction

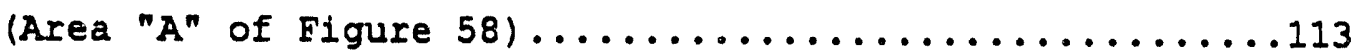


60. (OU1) Surface View GTA Rotational Weld Dendrites Along

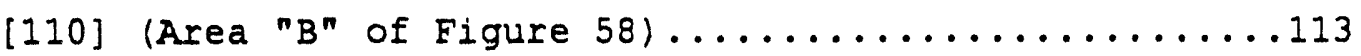

61. Transverse View [100] Direction GTA Rotational Weld High

Magnification of the Side Section (Area "C" of

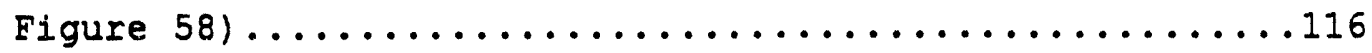

62. Three-Dimensional Reconstruction Identifying

Micrographed Areas.............................117

63. (001) GTA Rotational Weld in the As-Welded Condition

Dendrites in the [100] Direction, slip Lines, and Weld

Ripples (Area "An of Figure 62 ).................... 118

64. (001) GTA Rotational Weld in the As-Welded Condition

Dendrites in the [100] Direction, Slip Lines, and Weld

Ripples (Area "B" of Figure 62)........................

65. (001) GTA Rotational Weld Dendrites Along the <100>

Direction in the As-Welded Condition (Area "C" of

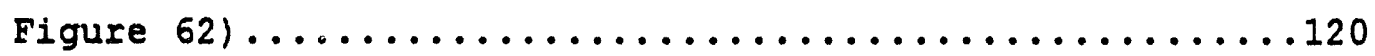

66. (001) GTA Rotational Weld Dendrites Along the <100> Directions in the As-Welded Condition (Area " $D$ " of

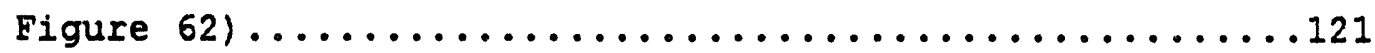

67. (001) GTA Rotational Weld in the As-Welded Condition Dendrites Along the [100] Direction and Slip Iines

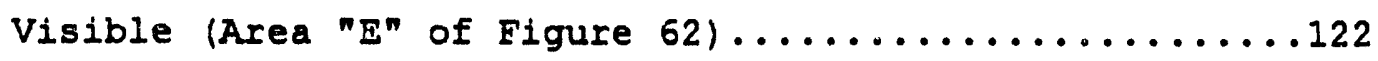


68. (001) GTA Rotational Weld in the As-Welded Condition High Magnification of Top Surface Dendrites (Area "F"

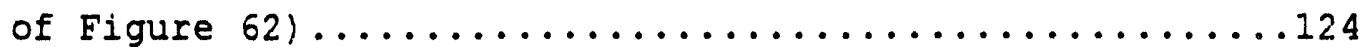

69. Three-Dimensional Reconstruction (011) Plane [100]

Direction Rotational GTA Weld with Accompanying

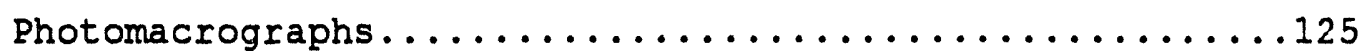

70. Three-Dimensional Reconstruction (011) Plane surface

Rotational GTA Weld with Accompanying Photomacrographs..126

71. Three-Dimensional Reconstruction (011) Plane [110]

Direction Rotational GTA Weld with Accompanying

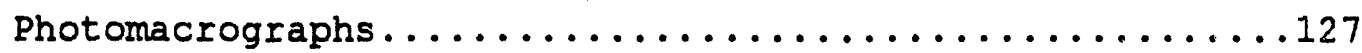

72. Three-Dimensional Reconstruction (011) Plane [111]

Direction Rotational GTA Weld with Accompanying

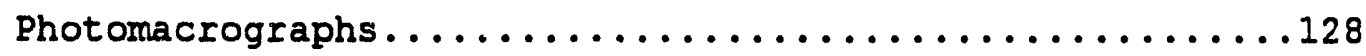

73. Three-Dimensional Reconstruction Identifying High

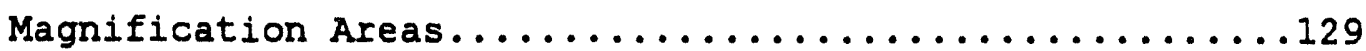

74. (011) Rotational GTA Weld symmetry in the Center of the Weld (Area "A" of Figure 73) .........................

75. (011) Rotational GTA Weld Dendrite Spacings (Area "B"

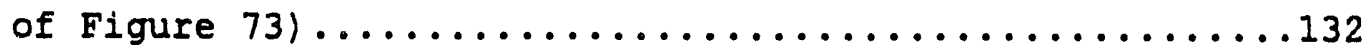

76. (011) surface symmetry of the Center of the Weld (Area

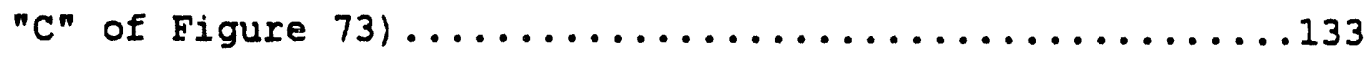


77. Three-Dimensional Reconstruction (111) Plane [110]

Direction Rotational GTA. Weld with Accompanying

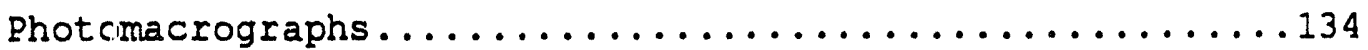

78. Polycrystal GTA Weld (a) Stationary (b) Rotational......136

79. (011) Plane [100] Transverse View GTA Weld

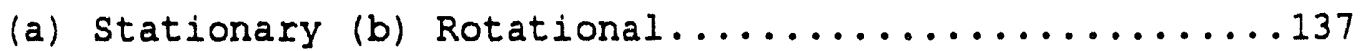

80. (001) Surface View GTA Rotational Weld of Two Melt

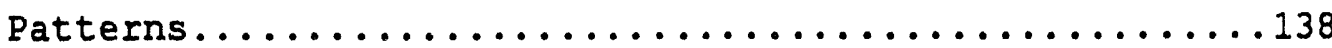

81. Profilometry Scan for the (001) surface..................

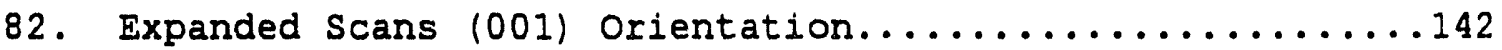

83. Data Plot of Amplitude Verses Position (001) Plane.......143

84. Profilometry Scan (011) surface........................

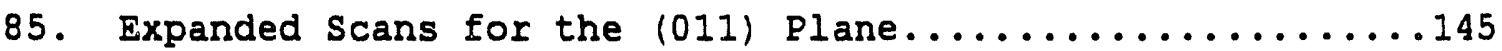

86. Data Plot of Amplitude Verses Position (011) Plane......146

87. (011) Plane Stationary GTA Overlapped Weld [100]

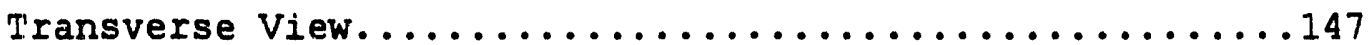

88. (011) Plane Stationary GTA Overlapped Weld High

Magnification of Bottom Section......................

89. (111) Plane Overlapped GTA Rotational Weld [110]

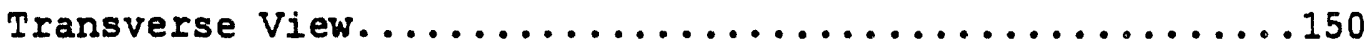

90. (111) Plane Overlapped GTA Rotational Weld High

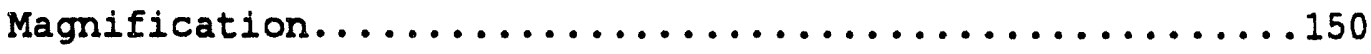


91. (011) Surface GTA $100 \%$ Argon Stationary Weld

(a) Surface View (b) [100] Transverse View............152

92. (011) Surface Stationary GTA $75 \%$ Helium and 25\%

Argon (a) Surface View (b) [100] Transverse View.......153

93. Stereographic Projection for the (001) Plane...........156

94. Schematic for (001) Plane EB Welding and sectioning

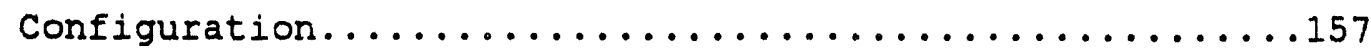

95. Three-Dimensional Recorstruction for the (001) Plane

Transverse Electron Beam Welded with Accompanying

Photomacrographs ............................ 158

96. Three-Dimensional Reconstruction for the (001) Plane

Surface Electron Beam Welded with Accompanying

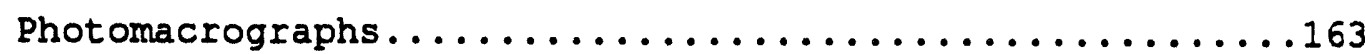

97. Transverse View of EB Weld $2^{\circ}$ Deviation from [100]

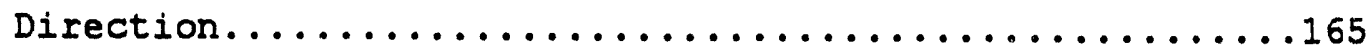

98. Three-Dimensional Reconstruction Identifying High

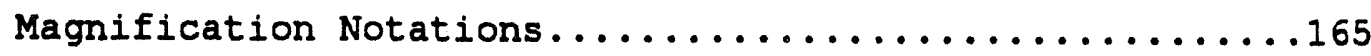

99. High Magnification of EB Weld [100] Transverse View

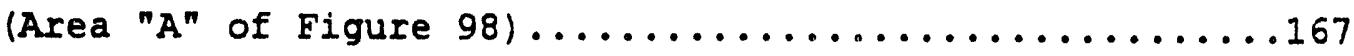

100. EB Weld (001) Surface View Along the [100] Direction.....169

101. EB Weld (001) Surface View Along the [110] Direction.....169

102. EB Weld (001) Surface Stray Dendritic Growth...........170 
103. Three-Dimensional Reconstruction of EB Welding (001)

Plane with Accompanying [110] Transverse Views........172

104. Three-Dimensional Reconstruction of EB Welding of (001)

Plane with Accompanying [100] Transverse Views........173

105. Weld Partially Melted Zone (a) Electron Beam Weld

(b) GTA Stationary Weld.......................

106. GTA Stationary (001) Surface Weld (a) (001) surface View

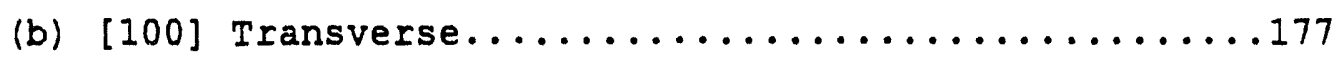

107. EB Weld (001) Surface (a) (001) surface View

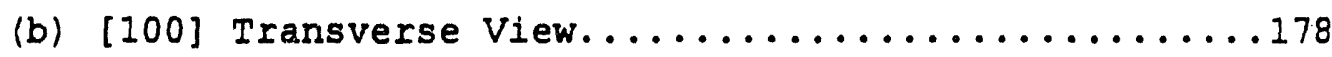

108. Polycrystal weld Placed in Various Positions...........190

109. Comparison of Weld Penetration for Multiple Welds

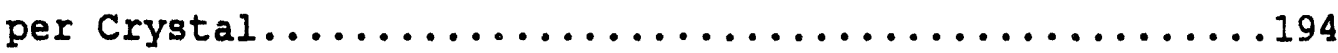

110. (001) Top Surface View of Clamped and Unclamped

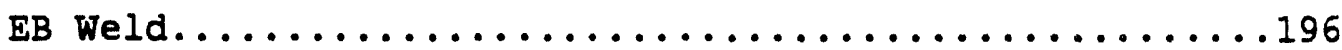

111. Unclamped EB Welds surface Views.......................

112. (001) Clamped surface Views $\mathrm{EB}$ Welds....................

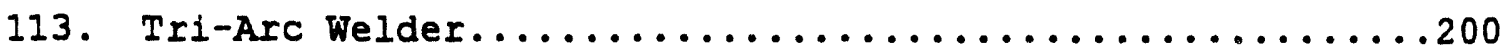

114. (011) Tri-Arc Weld Various Views...................... 201

115. Slip Iines on the Welded Crystal Surface (a) (001)

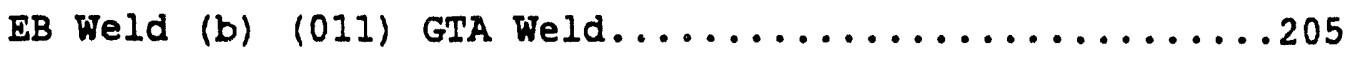

116. Data Plot for Hardness in the [100] Direction.........206

117. Data Plot for Hardness in the [010] Direction.........207

118. Data Plot for Hardness in the [110] Direction.........208 
IIST OT ABBREVIATIONS

\author{
Fe Elemental Iron \\ Ni Elemental Nickel \\ Cr Elemental Chromium \\ wt Weight Percent \\ RF Radio Frequency \\ kW Kilowatt \\ cfh Cubic Feet per Hour \\ GTA Gas Tungsten Arc \\ EBW Electron Beam \\ ppm Parts per million \\ FCC Face Centered Cubic \\ HCP Hexangonal Close Packed
}




\section{CHAPIER 1}

\section{INIRODUCTION}

The joining of materials through the process known as fusion. welding is accomplished by heating a common boundary of the two pieces to be joined above the melting points of the materials followed by cooling of the common molten region to form a union between the materials through the process of solidification. The molten pool (or "weld pool") is usually created between the two materials by heating with an electric or plasma arc, electron beam, laser, or by induction heating- although a number of other methods for forming a cormon molten boundary, such as friction or electric-spark discharges, are also utilized [1]. The fusion welding of materials is frequently accomplished by moving the heat source along the cormon boundary of the materials being joined so that the region between the materials is continuously being melted in front of the moving heat source and is continuously solidifying behind it to form a solid union along the entire boundary between the two parts. In one form of fusion welding, a stationary heat source can be employed so that the two materials are only joined at selected fixed points where the fusion and resolidification process takes place. Joining processes of this type are commonly referred to as "spot welding". 
The present investigation treats a simplified case of "spot welding" in which a molten pool is formed by a stationary heat source, but in this case, the molten pool does not form a common boundary with a second piece of material, and accordingly, the actual coalescence of two independent parts does not occur. This simplified form of "spot welding" was employed in order to realize a "model" configuration for the study of microstructural patterns formed by the solidification of high-purity metal alloys. In this work, the stationary melt pools were formed in oriented single-crystal specimens of an alloy of $708 \mathrm{Fe}-158 \mathrm{Ni}-$ 158Cr. The technique of using single-crystal-alloy specimens for investigating the properties of solidification microstructures has recently been pioneered by Rappaz, David, Vitek, and Boatner $[2,3,4,5]$ and has proven to be a powerful approach to obtaining quantifiable microstructural information. The investigations of Rappaz et al. $[2,3,5]$, however, have concentrated primarily on the case of fusion welds made in the conventional manner with a moving heat source.

In the investigations carried out in the present work, stationary melt pools were studied which were formed by a gastungsten-arc (GTA) heat source or by electron-beam (EB) melting. The GTA process involves heating by striking of an electric arc between the portion of the single crystal to be melted and a tungsten electrode [6]. In the case of the EB heating, a high- 
velocity beam of electrons is focused on the area of the sample to be melted [7]. The experimental configuration used to form stationary molten pools in the alloy single-crystal samples studied here is illustrated in Figure 1 . The experimental approach then proceeded by means of sectioning of the melt pool along predetermined crystallographic directions and microstructural analysis of the resolidified regions using standard metallographic techniques, the detalls of which are given in subsequent sections of this thesis.

\section{Bolidifioation Miarostruatures and Feld Properties}

The mechanical properties of welds are determined by their microstructural characteristics which, in turn, are governed by a number of parameters including the welding conditions such as the traverse speed of the heat source, the power input, welding atmosphere, the type of heating utilized, and by intrinsic characteristics of the base material such as its phase properties at various temperatures plus factors such as purity and crystallography. In particular, the crystallographic properties of the base metal or alloy, including the size and relative orientations of its component crystalline grains, play a crucial role in determining the nature of the grain-selection and growth processes that occur during the resolidification of the weld melt 


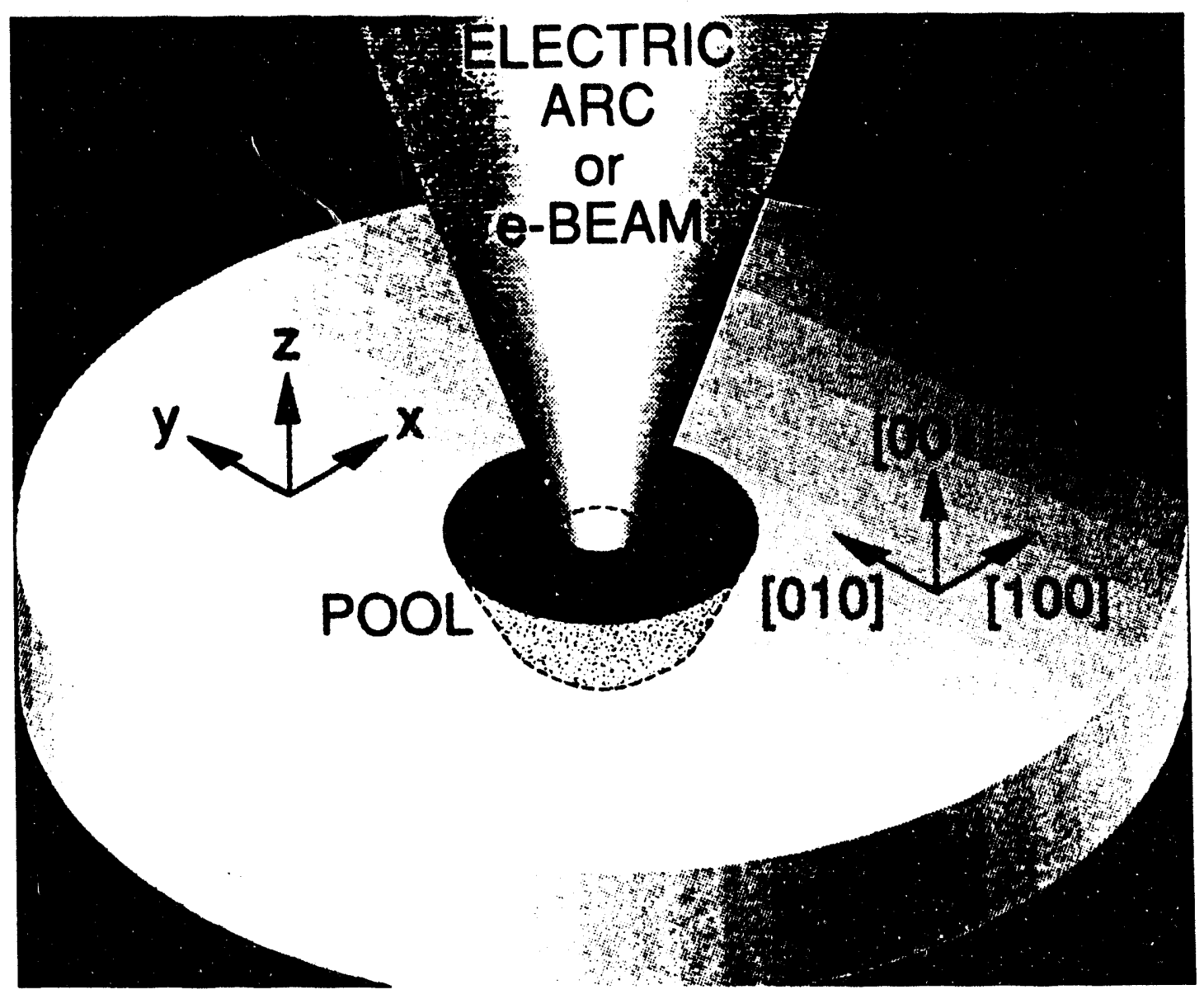

Figure 1

Schematic for spot Welding Configuration 
pool. In fact, the development of the solidification microstructure in a weld pool is primarily determined by the relative orientations of the melt-pool interface and heat-flow direction and the relative crystallographic orientation of the solid grains (or grain) on which epitaxial growth of the solidification cells is initiated at the solid-liquid interface that defines the shape of the weld pool $[3,8]$. In general, the growth of the solidification cells proceeds along so-called "easy growth" directions which are determined by the crystallography of the system. In the present case of studies of the fully austentic face-centered-cubic phase of a $70 \% \mathrm{Fe}-15 \% \mathrm{Ni}-15 \% \mathrm{Cr}$ alloy, the "easy growth" directions correspond to the <100> family of crystallographic axes.

\section{Research Background}

Prior to the relatively recent investigations of the solidification behavior and microstructural characteristics of EB and laser welds made using single-crystal base material $[2,3,4,5]$, almost all of the studies of solidification behavior in welding configurations have ut1lized polycrystalline base specimens that were either similar or identical to commercial materials. Such materials are, of course, characterized by a multiplicity of grains and orientations and by the presence of 
significant amounts of various impurity elements. During the solidification of the melt pools formed in complex alloy systems of this type, the initial epitaxial nucleation and growth of the solidification cells naturally takes place on randomly oriented grains, and this produces complex microstructures that are not amenable to quantification or to the development of analytical methods for describing the microstructural development. By replacing the usual polycrystalline weld specimens with highpurity oriented single-crystal samples, Rappaz et al. [2] have been able to develop an experimental welding methodology that results in reproducible and quantifiable solidification microstructures in which the roles of the heat flow and crystallographic effects were clearly delineated. These initial single-crystal welding investigations employed traditional welding procedures where the heat source (an electron beam or laser) was linearly translated across the weld specimen to produce a long, narrow melt path. For the case of a moving heat source, a melt pool is produced that is characterized by a relatively complex three-dimensional shape which is dependent on the welding speed. The well-defined microstructural information that could be extracted from the single-crystal weld zone subsequently led to the development of analytical approaches and the modeling of fusion-zone characteristics. By employing these analytical approaches, it was possible to predict the dendritic 
microstructure of a single-crystal weld from either experimentally determined or mathematically simulated weld-pool shapes.

\section{Purpose of Investigation}

In contrast to the previous investigations of Rappaz et al. $[2,3,4,5]$, the present work concentrates on the case of the melting and resolidification of a melt pool formed by a stationary heat source. The use of a stationary as opposed to a moving heat source results in the formation of a melt pool that exhibits axial symmetry rather than a complex asymetrical threedimensional shape. The object of this study is, therefore, to determine the microstructural characteristics of the more symmetrical fusion "spot welds" that can be formed in oriented Fe-N1-Cr ternary alloy single-crystals through the use of a fixed heat source. In pursuing this objective, solidification experiments have been performed in which the microstructural properties depended on various parameters associated with the weld-pool shape (1.e., the weld-pool cross section contour and depth). Two primary types of heat sources were utilized since it is known that EB melting produces a deep narrow weld pool with a non-circular depth profile cross-section while GTA melting results in the formation of stationary melt pools whose cross- 
sectional profile is more circular. Both EB and GTA melting were applied in the formation of melt pools on the pure $708 \mathrm{Fe}-158 \mathrm{Ni}-$ $158 \mathrm{Cr}$ alloy single-crystals. These crystals were grown by the Czochralski method and oriented so that the melt pools were formed on (001), (011), and (111) planes. In some cases, the single-crystal samples were rotated about an axis that corresponded to the center of the heat source in order to carry out an effective averaging of potential thermal inhomogeneities. By using the two types of heat sources in the formation of the stationary melt pools, it was possible to prrice molten regions in the single-crystal specimens that exhibited distinctly different solidification microstructural patterns. By subsequently performing microstructural analyses based on standard metallographic methods including optical microscopy. The metallographic observations were used to produce a threedimensional reconstruction of the resulting microstructure that was characteristic of each type of heating and of each crystallographic orientation of the single-crystal specimens. The plesent results provide the basis for the future development of analytical methods for the calculation of the threedimensional misrostructural properties for a varlety of crosssectional contours characteristic of stationary melt pools that are formed in oriented single-crystals. 


\section{CEAPTER 2}

\section{IITERATORE REVIEW}

In order to develop the conceptual basis for the research effort described here, a number of topics related to stainless steel single-crystal welding and to microstructural analysis were surveyed. In particular, literature concerning the following areas of study was reviewed: 1ron-nickel-chromium alloy systems, general weld-pool melting and solidification, stainless steel single-crystal $\mathrm{EB}$ welds, and single-crystal welds for alloys other than stainless steels.

\section{Iron-Niake1-Chromium N10ys}

The phases present in type 308 stainless-steel has been studied zapffe and Vitek, Dasgupta, and David $[9,10]$. According to research performed by Vitek, Dasgupta, and David [10], for the ratio of $\mathrm{Cr}_{\mathrm{eq}} / \mathrm{Ni} \mathrm{eq}^{<1.35}$, where $\mathrm{Cr}_{\mathrm{eq}}=\mathrm{Cr}+\mathrm{Mo}+1.5 \mathrm{S1}$

$$
\mathrm{Ni}_{\text {eq }}=\mathrm{Ni}+30 \mathrm{C}+0.5 \mathrm{Mn}
$$

the stainless steel remained as primary austenite after solidification. If the ratio was greater than 1.35 , then the resulting resolidified zone contained primary ferrite. In this 
investigation the ratio is 1.0 which means that this stainless steel will be fully austenite. They also found that a fully austenitic stainless steel remained fully austenitic independent of the cooling rate $[10]$. A vertical section of the $\mathrm{Fe}-\mathrm{Ni}-\mathrm{Cr}$ phase diagram is illustrated in Figure 2 [10]. This illustrates, for the composition used in this investigation, that the material is fully austentitic.

\section{rield Pool}

The melting processes influence the weld pool geometry. Because of the relationship of the weld-pool shape and the resultant microstructure, these melting processes also greatly influence the solidification microstructure in the fusion zone. They also affect the defect structures in the weld including porosity, inclusions, and hot cracking phenomena [8].

\section{Melting}

Among the factors that influence the development of the weld pool are the heat input, fluid flow (i.e., mass transport) in the molten pool, and heat transfer in both the weld pool and the base metal. Fluid-flow phenomena affect both the temperature and cooling rates in the entire weld pool thereby influencing the 


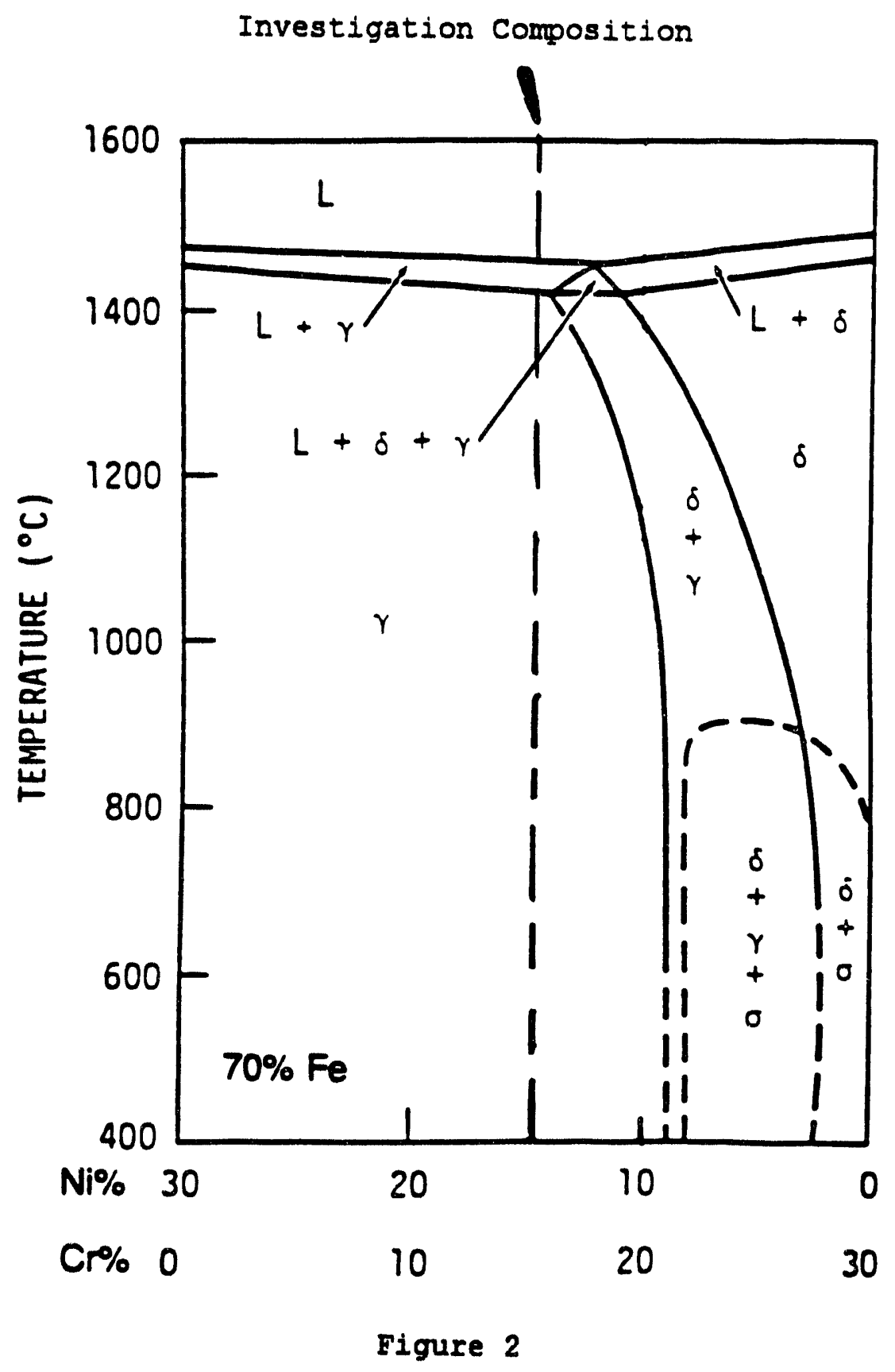

Stalnless steel Phase Diagram 
final microstructure of the weld pool [11]. These are critical processes that control the penetration of the molten pool into the weld specimen. Several factors have been determined to enhance or counteract convection effects in the weld pool. These are surface tension, buoyancy, and electromagnetic forces [12]. Depending upon the interaction of these forces, penetration of the weld could increase or decrease [11]. Previous studies have shown that the GTA welding method produced electromagnetic-field effects that reduced the fluid flow by diminishing the effects of surface tension. This effect became evident when a laser spot weld of the same material produced a smooth fluid flow. The smooth flow resulted only from the bouyance and surface tension forces reaching a quasi-steady state [13].

\section{Solidification}

Growth rate, undercooling, and alloy composition in the solidification process are important parameters in the development of the microstructure [14]. The fusion-zone microstructure is primerily controlled by the base-metal microstructure, by the influence of crystallography which favors preferred growth directions, and by weld-pool shape [14]. Solidification occurs initially by spontaneous growth at the weld boundary, 1.e., at the solid-liquid interface. This spontaneous 
growth involves a special case of heterogeneous nucleation in which there is no nucleation barrier $[14,15]$.

After the initial solidification occurs, epitaxial dendritic growth continues from the edge of the weld to its center $[14,15]$. Dendritic growth can be characterized as "competitive growth" because there is only one active growth direction in a given location. This active dendritic growth occurs along the preferred orientation, 1.e., along the direction most closely aligned to the heat-flow direction. However, this directionality does not mean that only one set of favored growth directions exists over the entire weld microstructure. If the heat source changes the weld-pool shape, then the maximum thermal gradient is altered to produce a new preferred dendritic orientation; however, there is only one active growth direction occurring in a given location [16].

The interface shape and stability depends on the exact thermal and compositional gradients in the vicinity of the solid1iquid interface. These gradients are affected by the alloy composition which affects the solid/liquid interface stability. The alloy affects the development of the microstructure by influencing the solute redistribution. Based on the actual thermal and compositional gradients, the possibility of an insufficlent undercooling would cause the solid/liquid interface to remelt. Other possible interactions between the exact thermal 
and compositional gradients allow the solid/liquid interface to propagate through the weld [16]. Although the heat source is turned off in these stationary welds during solidification, consideration still needs to be given to the fact that the solidliquid interface temperature gradient is lower for the stationary welds compared to the translational welds [17].

\section{8tainless steel single Crystal Welding}

\section{Translational Welds}

The research performed by Rappaz, David, Vitek, Boatner $[2,3,5,18]$ was carried out by using high-purity austenitic stainless-steel single crystals of the ternary alloy composed of 708Fe-15\%Ni-15\&Cr (weight percent). The first study represented a microstructural investigation that utilized full-penetration translational EB welds along the [100] and [110] directions on the (001) surface of the oriented single-crystal samples (Figure

3) [3]. This investigation reached these conclusions:

1. After welding, the weld was still effectively monocrystalline in nature as verified by the Laue backreflection techniques.

2. Dendritic growth occurred parallel to the three <100> directions.

3. Crystallography influenced the dendritic growth. 


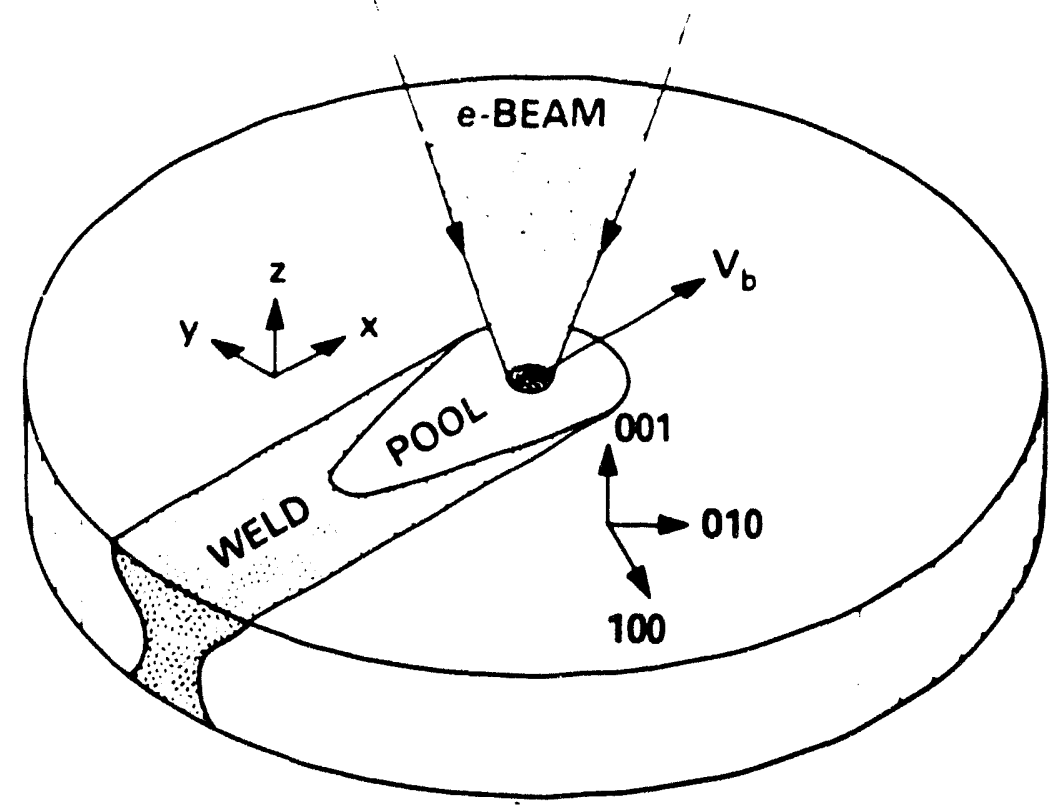

Figure 3

Schematic for Translational Electron Beam Welds

4. The specific $\langle 100\rangle$ orientation that would survive and propagate was influenced by the weld-pool shape and the heat-flow direction.

The nature of the dendrite-growth-selection process was determined from a dendrite-velocity analysis. The dendrites that were most closely aligned with the minimum velocity survived because the required amount of undercooling for these dendrites was less compared to faster velocity dendrites. Using this 
information and micrographs of top and transverse views, the researchers were able to depict the solidification microstructure in a three-dimensional fashion (F1gure 4) [3]. In this figure $V_{b}$ is the velocity of the heat source, a and $e$ are regions of [100] dendrites, $b$ and $d$ are regions of [010] dendrites, and $c$ is [001] dendrites.

A second investigation by David, Vitek, Rappaz, and Boatner [2] presented an analysis of the weld-pool shape and microstructure as a function of welding speed. Figure 5a [2] represents the case of a full-penetration low welding speed, 4.2 $\mathrm{mm} / \mathrm{s}$, in which the dendrites grew along the [100] and [010] directions. Figure 5b [2] illustrates a full-penetration high welding speed, $42 \mathrm{~mm} / \mathrm{s}$, in which the dendrites were similar to those observed for low-speed growth - except for an increase in the amount of [001] dendrites near the surface of the weld. A comparison of the two welds revealed that the weld crown was less distinct and the weld-pool shape was more elongated for the higher-speed welding case. Also, at the higher welding speed, dendrite branching diminished. These experiments also showed that the weld-pool shape formed when using the same welding speed were similar from weld-to-weld independent of the crystallographic directions. This formation demonstrated that the weld-pool shape is not determined by crystallography, but rather by the thermal properties of the material. 


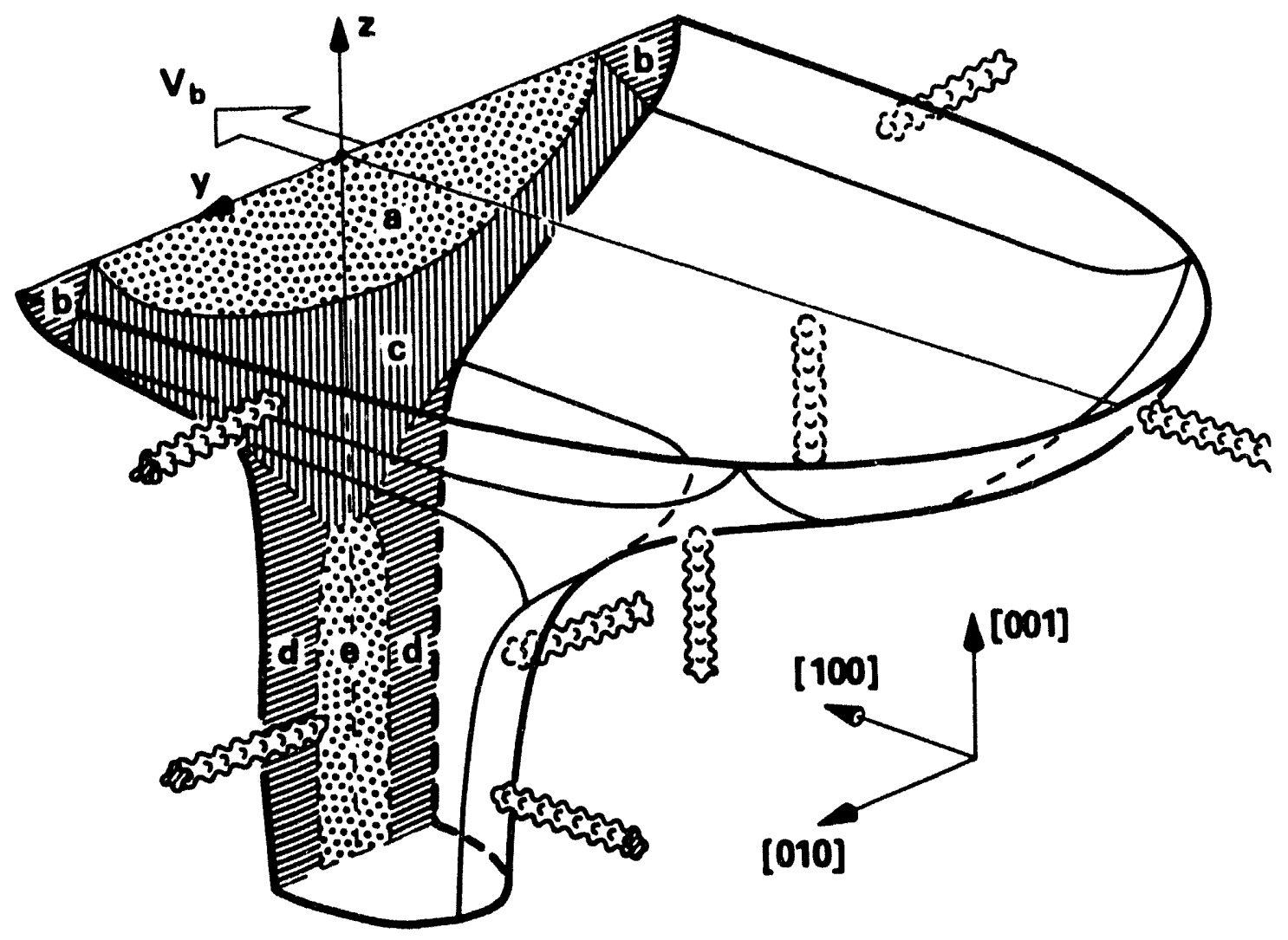

[100] WELD $3 \mathrm{~mm} / \mathrm{s}$

Figure 4

3-Dimensional Model for Translational Electron Beam Weld 


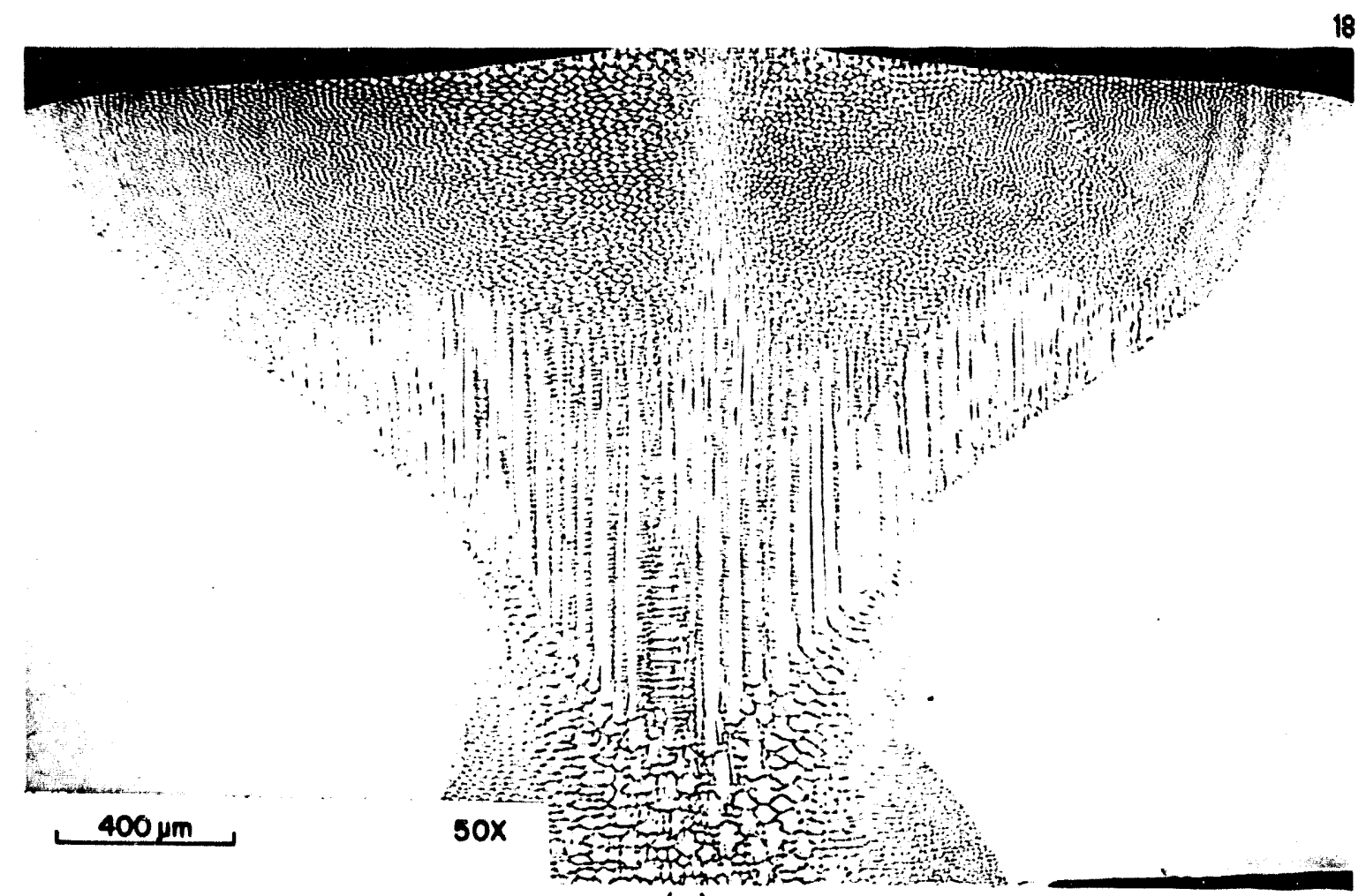

(a)

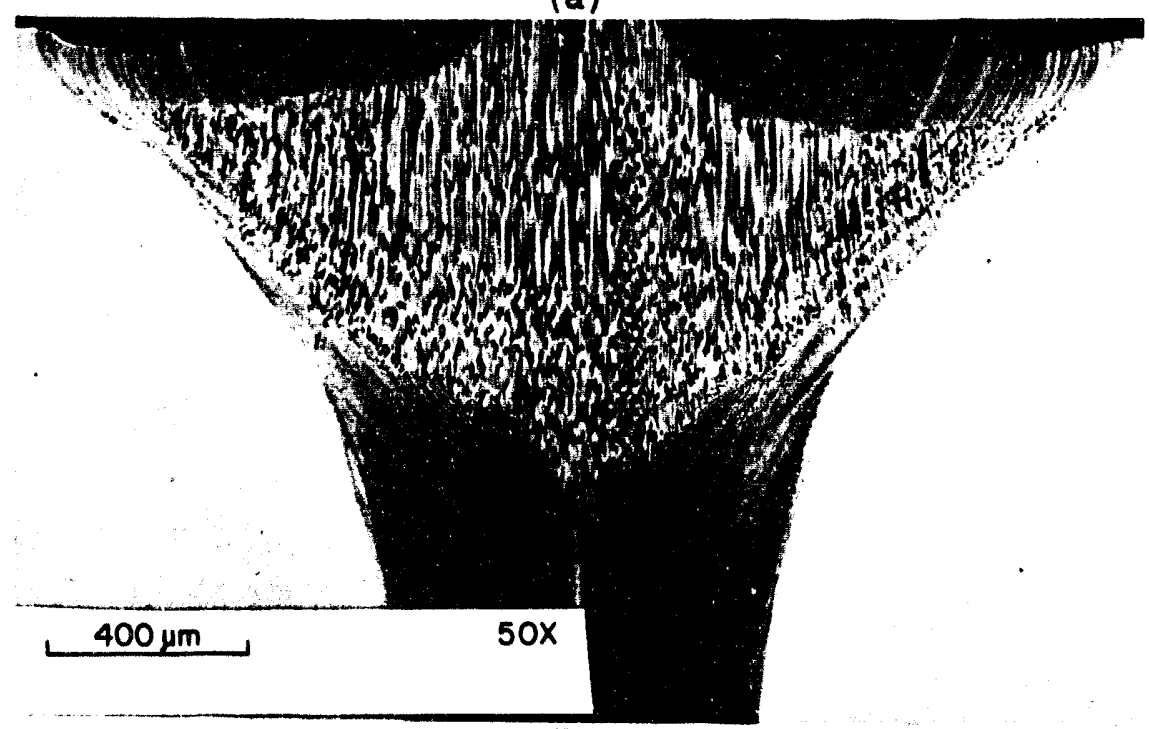

(b)

Figure 5

Welding (001) surface Along [110] Direction with

Translational speed of (a) $4.2 \mathrm{~mm} / \mathrm{s}$ (b) $42 \mathrm{~mm} / \mathrm{s}$ 
An extension of the research carried out by Rappaz et al. [18] employed mathematical models to predict the resulting microstructures which were then compared with the experimental results obtained in their previous research. This model utilized the dendrite-growth velocity which involved the relationships between the welding direction and the melt-pool surface normal unit vectors, as well as the direction and velocity of the heat source. Also, the "best alignment" criteria, where the dendrite growth more closely aligned to the solidification-front normal will have the smallest undercooling, was employed in this model. The growth velocity of the dendrites is given by the equation:

$$
\left|V_{h k I}\right|=\left|V_{b}\right| \cos \theta / \cos \Psi
$$

where: $V_{h k l}$ is velocity of the dendrite

$v_{b}$ is the velocity of the heat source

$\theta \quad$ is the angle between the heat source and the solidification front

$\Psi \quad 18$ the angle between the dendrite direction and the solidification front direction.

This equation 1llustrates that each favored growth direction will not have the same growth velocity as an other preferred growth direction in the welding system because of the variations in the angles [2]. The results showed that this growth will result in 
the elimination of all other dendritic growth. The model also used the dendritic-growth theory of Bobadilla, Lacaza, and Lesoult [19] to calculate the dendritic-growth patterns in the welds. A comparison of the predictions of the mathematical model to the experimental results (as obtained from their earlier experiments) revealed very good agreement. This established the capability of both the experimental and the mathematical methods for carrying out an analysis of single-crystal microstructures. A final extension of this research involved using an arbitrary crystallographic weld surface and weld direction [5]. This work verified that reasonable agreement between the theoretically predicted and the experimentally determined microstructures was possible. From these experiments, a capability resulted that allowed for the prediction of the resulting microstructure of a translational EB weld on stainless-steel single crystals.

\section{Multipass Welds}

Other research on stainless-steel single crystals reported by David et al. [2] represented studies of overlapping multipass partial-penetration welds created by EB welding at a speed of $\mathbf{4 . 2}$ $\mathrm{mm} / \mathrm{s}$ (Figure 6) [2]. The samples were of the same composition as that used in the previously mentioned stainless steel singlecrystal welding experiments. It was found that the dendritic growth and the weld-pool shape were repetitive for each pass and 


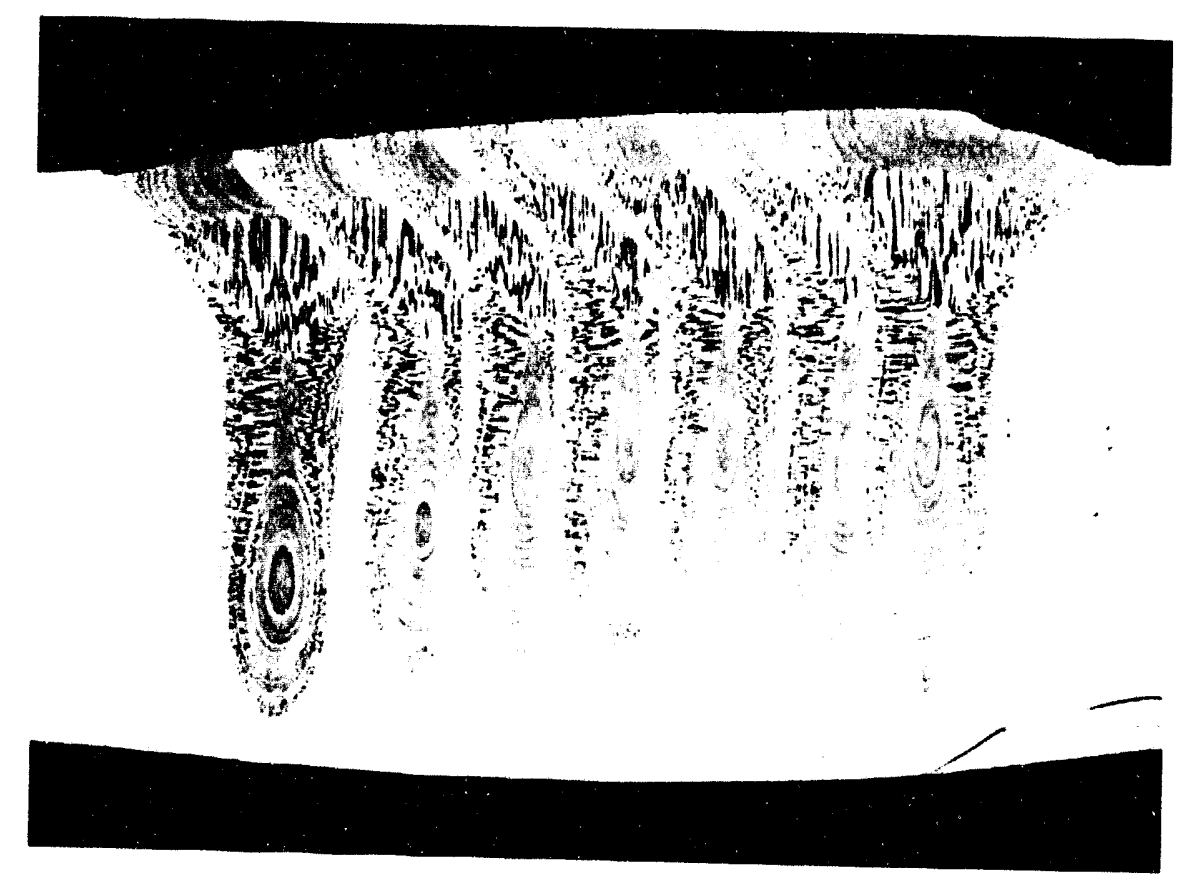

Figure 6

Multipass Translational Electron Beam Welds $4.2 \mathrm{~mm} / \mathrm{s}$ Along the [110] Direction (001) Surface

from sample to sample. However, the overall microstructure more closely resembled that produced by a $3 \mathrm{~mm} / \mathrm{s}$ welding speed. The main reason for this was the heat flow in the crystal.

Two factors affected the heat-flow patterns: the partial penetration of the welds, which was a parameter of the experiments, and the elevated sample temperature, which was caused by the consecutive mitiple welds. An important discovery was that the dendritic growth depended on the crystallographic 
orientation of the system and was independent of the pre-existing dendritic nature of the previous weld pass.

The partial-penetration effect of each weld pass produced "striations" in the microstructure which were evident at the bottom of the weld pool. These striations indicated that there were weld-pool variations.

Bicrystal Welds

David et al. [4] and Vitek et al. [20] have carried out adifitional welding studies in which the surface of two crystals of stainless steel of the same composition, but with different crystallographic, orientations, were joined. The goal of these studies was to determine the interaction between the two crystals. These investigations involved three different welding configurations. The first consisted of a (001) surface cut along the [100] direction that was then "butt" welded to a (001) surface cut along the [110] direction. The second involved a butt weld of a (011) surface cut along the [100] and [011] directions. The third configuration used a weld made along the [211] direction on the (011) surface butt welded to the (001) surface along the [100] direction.

For the initial experiment (1.e., the (001) surface butt weld of the [100] and [110] directions), it was determined that each haif of the weld microstructure resembled that of 1 ts 
respective single-crystal microstructure [2]. The second experiment utilized two crystals with the (011) surface butt welded along the [100] and [011] directions. These experiments employing bi-crystals revealed that the grain growth was competitive and that growth velocity was still an important factor. The growth of one crystal into the other in some areas of the weld pool established this phenomenon. By using the criteria stated previously, the dendritic growth with the lowest undercooling eliminated the other possible preferred dendrite growths.

The third bi-crystal experiment involved translational EB welds on different surfaces and different directions. These welds joined the (011) surface along the [211] direction to a (001) surface along the [100] direction. The dendritic growth in each single-crystal half resembled the normal growth patterns as in a single-crystal weld. One crystal again grew into the other in small sections of the microstructure at the weld centerline confirming again the influence of growth velocity and undercooling on crystal-growth direction.

A mathematical model using the dendritic-growth velocity and the minimu-dendrite-tip undercooling criteria was developed to predict the resulting microstructure for the bi-crystal experimental work noted above. A comparison of the model to the 
experimental data showed a good agreement between the theory and the experimental data.

\section{single Crystal welding}

\section{Copper single Crystal Welds}

Copper single crystals were used previously by savage, Lundin, and Chase [21] in early studies which investigated solidification phenomena in welds. Three distinct factors were found to influence the solidification patterns: alloy composition, temperature gradient, and growth rate. One factor which influenced the temperature gradient was the weld-pool shape. If the weld-pool shape remained constant throughout the weld, competitive growth in the cells was negligible. However, if the pool shape changed, then differences in the cellular structure enhanced the possibility that the growth direction would also change. Realignment with the maximum-temperaturegradient direction caused this dendritic alteration.

\section{Aluminum Welds}

Some related work by Nakagawa, Katoh, Matsuda, and Senda [17] on the modeling of spot welds used pure coarse-grained or singlecrystal aluminum sheets of varied chemical purity. Their procedure employed a GTA process coupled with standard 
metallographic characterization of the microstructure. The study found that the cellular dendritic arm spacing was approximately a constant value; however, this spacing increased with increased arc time. Growth of the columnar grains decreased as arc time increased. This study also revealed that the microstructure of the weld pool changed from a cellular one at the periphery to a cellular-dendritic and then to an equiaxed-dendritic configuration at the center of the weld pool [17].

other studies by Nakagawa Kato, Matsuda, and Senda $[22,23]$ on solidification structures of aluminum determined:

1. After welding a single crystal, the weld metal was still effectively a single crystal with the same orientation as the parent material.

2. The preferred-growth directions for aluminum (facecentered cubic) was along the <100> directions.

3. Even with high cooling rates, as under welding conditions, the preferred growth directions were maintained.

4. If there was no preferred-growth direction, the dendritic growth was parallel to the direction of the maximum thermal gradient.

An extension of this welding research involved the joining of two crystals with different orlentations [24]. These aluminum crystals demonstrated the same growth patterns as those observed 
for the stainiess steel single crystals $[2,20]$, and growth from one crystal impeded the growth of the second crystal. These researchers attributed this effect to the alignment of thermal gradients favoring one growth direction over another, thus presenting the competitive-growth aspect of the crystals.

A study by Nakagawa et a1. $[25,26]$ that was concerned with the growth of stray crystals during the solidification process used GTA welding of 99.938 pure and commercially pure aluminum single crystal and coarse-grained materials. Stray crystals formed if the maximum temperature gradient deviated from the nearest $\langle 100\rangle$ direction by between $40^{\circ}$ and $50^{\circ}$. If the sample purity declined, the higher the purity of the material, the lower the probability for stray dendritic growth. A correlation between the welding speed and stray growth was observed. Lower welding speeds produced more stray-growth dendrites [26]. New dendritic growth occurred along the $\langle 100\rangle$ preferred directions. Researchers have recently investigated the nucleation mechanisms and convection effects in the weld-pools in order to aid in the explanation of these phenomena $[16,25,27]$. Dendrite fragmentation caused by convection in the weld-pool was found to produce new grains. The tip of the dendrite can break off, flow into the melt, and nucleate as a new dendrite $[16,25]$. The second mechanism, similar to the first, involved the detachment of an entire grain instead of a dendrite tip. Heterogeneous 
nucleation, the final mechanism considered, was ruled out as a source of stray crystals because difficulties in overcoming critical energy barriers made the conditions not conducive to new growth [27].

\section{Aluminum and zinc Welds}

A study by Passaja and Bakish [28] employed EB welding on aluminum and zinc single crystals. These workers concluded that preferred dendritic growth occurred along the <100> directions for FCC aluminum and along the <0001> directions for HCP $z$ inc. Noted in this study were variations in the dendritic-growth rates. The resulting microstructure formed "step-like" features that were caused by uneven dendritic growth in all areas of the weld. Impurities, even in the 99.938 high-purity crystals, impeded dendrite growth during solidification.

\section{mold RApples}

Surface ripples which appeared on welds required further research in order to determine the process by which ripple formation occurs. Several theorles presently exist concerning the development of these surface ripples $[29,30]$. Copley, Beck, Esquivel, and Bass [29] have concluded that surface ripples are caused by convective fluid flow in the weld-pool. This is 
explained by the fact that when the temperature is highest, the surface tension is lowest, and that the liquid level was lowest under the beam. As the distance from the beam increased, the temperature decreased so that the surface tension increased along with the liquid level. From the stated temperature differentials and the liquid height differentials from the center of the weld outward, a pressure head develops and induces a counterflow toward the lower liquid level, resulting in an elevated region of molten metal. A steady-state condition (reached by a balance of the counterflow with gravity) was followed by solidification to form the ripples [30]. Figure 7 [29] 1llustrates a comparison of the convective patterns for stationary and translational welds. This figure shows that for a stationary melt, the convection pattern is symetric about the center of the weld resulting in a more symmetric ripple pattern $[29,31]$.

\section{Bummaxy}

The preceding discussion summarizes the background information in the pertinent areas of weld-pool melting and solidification, welding of stainle-s-steel single-crystals, welding of other single-crystal materials, and finally for the formation of weld "ripples". The research on stainless-steel single crystals determined that dendrite growth depends on the 

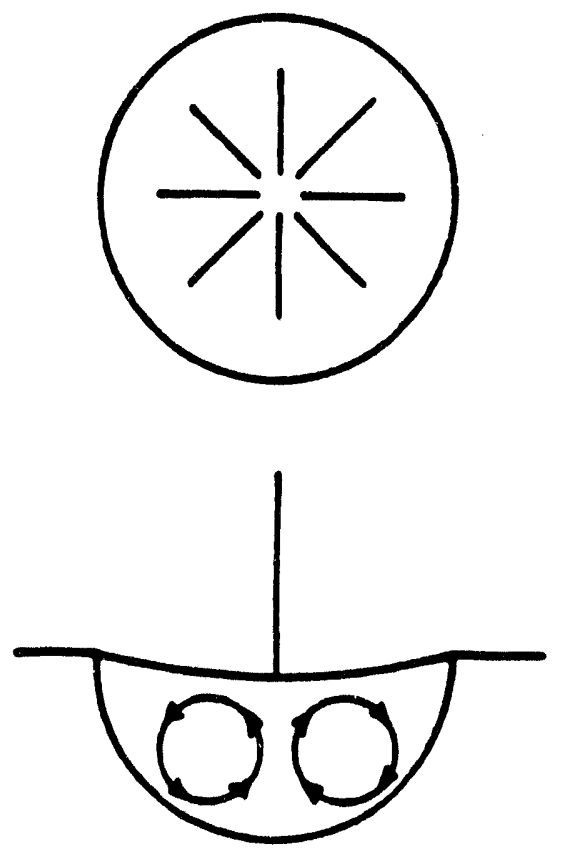

(a)
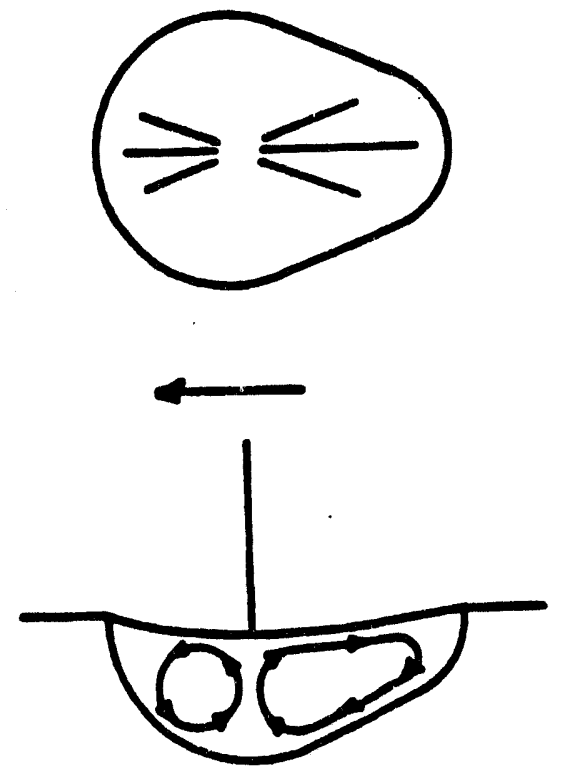

(b)

Figure 7

Convection in a Laser Melt for a Scan Velority

(a) Zero (b) Greater than Zero 
weld-pool shape and crystallography. This was also concluded from the other welding experiments on the other materials. In the case of bicrystal welds it was found that the dendritic growth impeded the growth from one crystal into the other depending on the dendrite velocities. The formation of ripples on the solidified weld surface was associated with convective flow in the molten weld-pool. The present literature survey serves to indicate how the current research on the microstructural properties of stationary melt pools in stainless steel single-crystals can add to our present understanding of solidification phenomena and microstructural development. 


\section{CEAPTHR 3}

\section{EXPLRTMATIAT PROCEDORES}

The objective of the present investigation is to determine the microstruitural characteristics of fusion welds in which the welding heat source is held stationary, thus creating a type of "spot weld" on oriented surfaces of Fe-Ni-Cr ternary-alloy single crystals. Accordingly, the experimental approach of this investigation initially required the growth of oriented stainless-steel single crystals with the composition 708 Fe-158Ni$158 \mathrm{Cr}$ (wty) for the spot welds. These crystals were grown by the Czochralski method, in which an orlented "seed" was used to "pull" the cryatal from a melt contained in a crucible. Specimens with the $(100),(110)$, and (111) planes were prepared, and the orientation was verified by means of the Iaue backreflection $X-r a y$ technique. This technique was also used to determine the primary directions in the plane of the crystal. GTA and $E B$ melting were used to form the atationary melt pools. In some cases the crystal was rotated about the heat source in an attempt to produce a more uniform weld-pool shape by eliminating thermal inhomogeneities in the electric arc. A more uniform weld-pool shape would, of course, result in a higher degree of symetry in the resultant microstructure. 
After careful sectioning of the spot weld along

predetermined crystallographic directions, standard

metallographic procedures were used along with optical microscopy to obtain quantifiable information that was then used to carry out a three-dimensional reconstruction of the solidification microstructure. The details of the experimental procedures used for the preparation of the crystals, welding, and weld characterizations are presented below.

\section{Cryotal Growth}

The high-purity stainless-steel single crystals used in this research were grown by the Czochralski method [32], in which an oriented seed crystal was used to nucleate growth along a desired crystallographic orientation. Subsequently, the crystal was slowly "pulled" from the molten metal pool using a variable speed motor. As the crystal was "pulled" from the melt, solidification took place in the plane of the orientation of the rectangular seed crystal. The ternary-alloy melt was prepared in a multipurpose growth chamber using a $30 \mathrm{~kW}$ generator, and the . growth was carried out in a purified-argon atmosphere at a pressure of 1 AMM. The crucible for the molten metal was highdensity aluminum oxide, which was held inside a graphite receptor. The alloy composition (708Fe-158N1-158Cr (wt 8$)$ ), was 
prepared using high-purity (99.998) elemental iron, nickel, and chromium. The composition of the alloy was verified by mass spectroscopy (Table 1). The homogeneity of the crystal was maintained throughout by mixing of the melt. Mixing of the melt was caused by the radio frequency (RF) current and by counter-

Table 1

Impurity Elemental Analysis of Stainless steel Single Crystal

\begin{tabular}{|c|c|}
\hline Element & Percent of Element \\
\hline $\begin{array}{l}\text { Carbon } \\
\text { Phosphorus } \\
\text { Suliur } \\
\text { Sticon } \\
\text { Nickel } \\
\text { Chromium } \\
\text { Cobed } \\
\text { Aluminum } \\
\text { Owygen } \\
\text { lion }\end{array}$ & $\begin{array}{c}0.004 \\
0.003 \\
0.002 \\
0.02 \\
16.71 \\
14.27 \\
0.02 \\
0.003 \\
0.003 \\
\text { Bdence }\end{array}$ \\
\hline \multicolumn{2}{|c|}{ 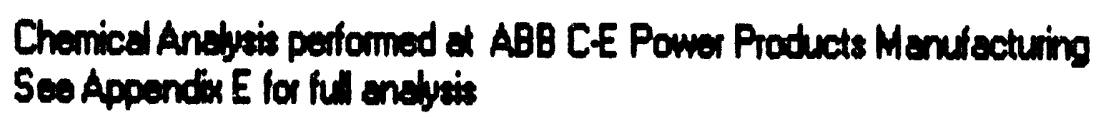 } \\
\hline
\end{tabular}


rotating the seed and crucible during the pulling motion of the crystal, from the melt. To confirm the absence of any stray crystals the end of the large crystal or boule (Figure 8) was etched for approximately thirty seconds with a mixture of 758 hydrochloric acid and $25 \%$ hydrogen peroxide. The resulting single crystal boule was then sectioned using an electric-arcerosion cutter to form slices approximately $0.3 \mathrm{~cm}$ thick (Figure 9). The crystal orientations were determined to within $1^{\circ}$ by using a North American Philips Norelco X-ray generator equipped with a Laue back-reflection $X$-ray diffraction camera. The X-ray system was operated at $30 \mathrm{KV}$ and $20 \mathrm{~mA}$, with a specimen distance of $5 \mathrm{~cm}$ from the camera. Laue back-reflection $\mathrm{X}$-ray diffraction was used to confirm the orientation of the plane in several areas of the specimen to further verify that only one single grain was present in the crystal. If any deviation from the desired plane of the single crystal was observed, then the sample was lapped and polished while held in a sample orientation goniometer to correct the divergence. The samples were then given a final polish with a colloidal silica mixture on a polishing lap to produce a smooth surface. 


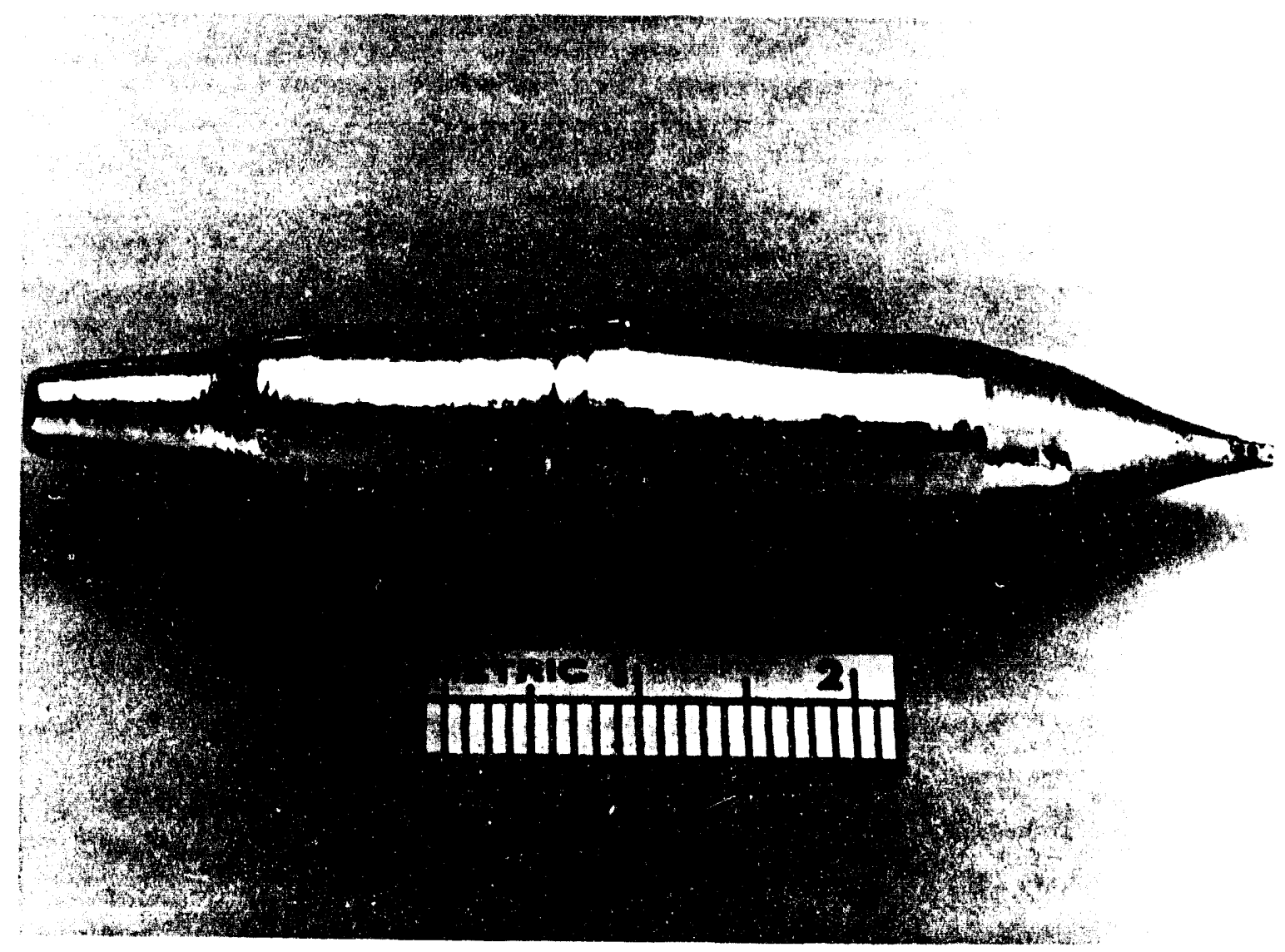

Figure 8

Crystal Boule 


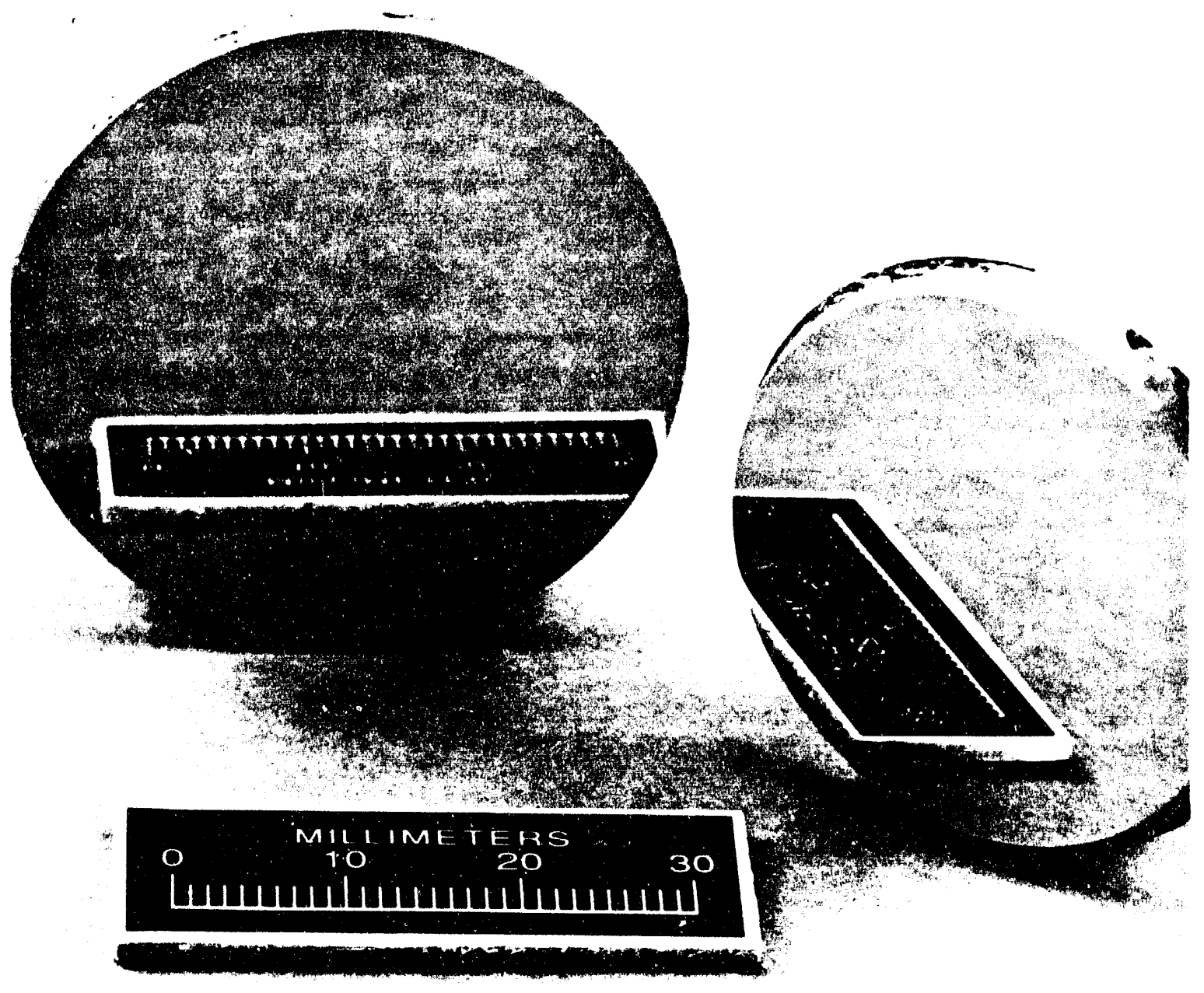

Figure 9

Sectioned Crystals 


\section{melding}

In welding the single crystals, the goal was to produce a spot weld on the stainless-steel specimens in which the molten pool penetrated 80 to $90 \%$ of the sample thickness. Two types of heat sources were employed, as described in the following section.

\section{Electron Beam Welding}

Using a Leybold-Heraeus $15 \mathrm{~kW}$ EB welder (Figure 10), spot selds were created on the single crystals. These welds were performed with the sample in the horizontal position using the parameters given in Table 2. The beam current was adjusted to

\section{Table 2}

Electron Beam Welding Parameters

Electron Bean Wolding Peameters

Working diatance $\quad 30.5 \mathrm{~cm}$ Acceler ding Volkege $100 \mathrm{Klow}$ Beam current $\quad 5.0$ miliemps Focel Cunenk 0.600 Vocum $10-6 \mathrm{~Pa}$

Wedd Time 2.6 seconds 


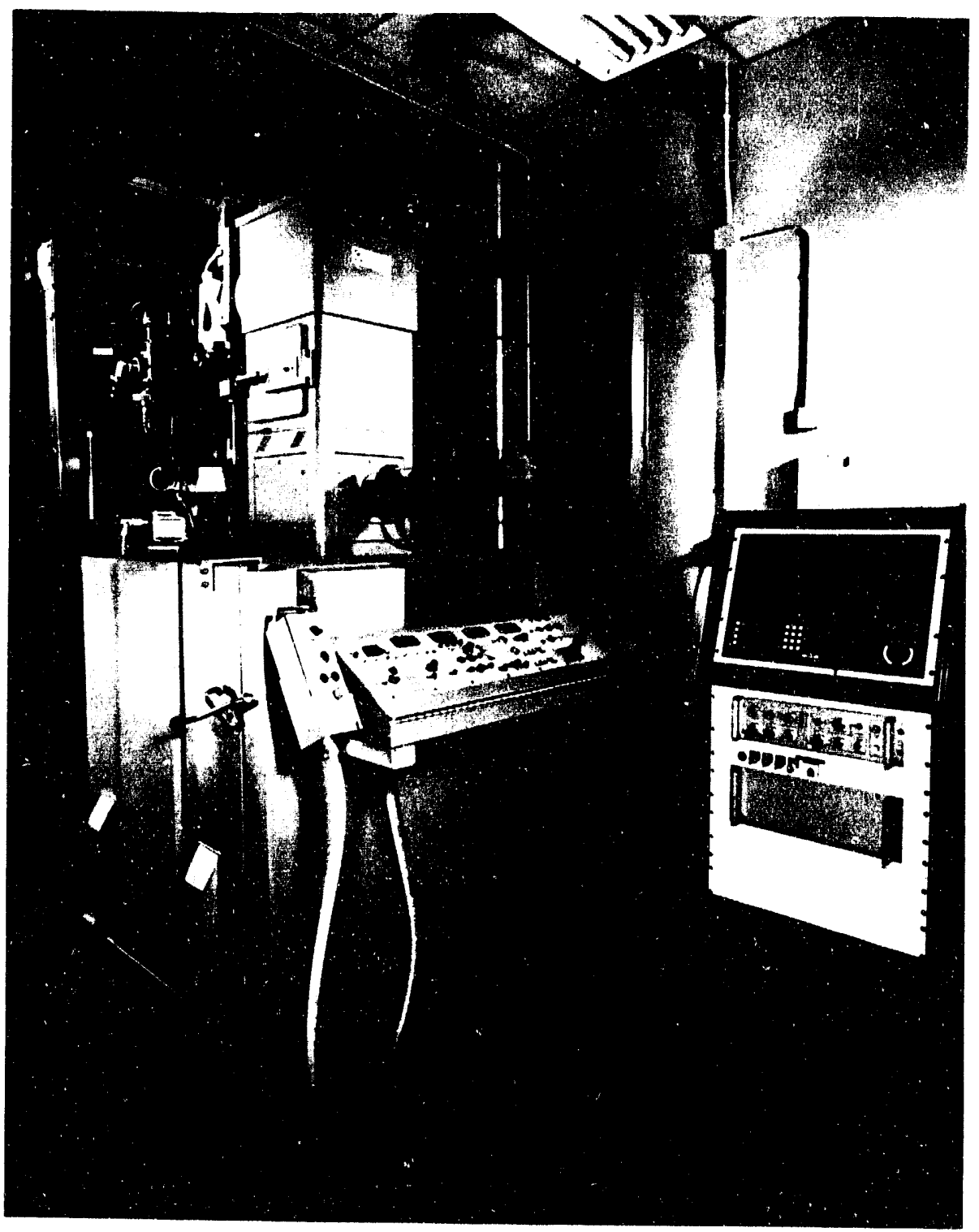

Figure 10

Electron Beam Welding system 
allow for variations in the sample thickness and to avoid the production of a full-penetration weld. Depending on the diameter of the crystal, miltiple spot welds were made on a single-crystal disc. In these cases, there was a time delay of approximately five minutes between welds in order to allow the sample to cool and thereby avoid any preheating effects.

Attempts were made to produce a more-uniform circular spot weld by adjusting of the beam focus and beam current, and by the use of electronic "circle generation" of the beam at frequencies from $1 \mathrm{~Hz}$ to $1000 \mathrm{~Hz}$. EB welds usually formed an oblong weld pool shape instead of a circular shape. Attempts to achieve a uniformly circular weld-pool shape were generally unsuccessful.

\section{Gas Tungsten Arc Welding}

The GTA welding process (Figure 11) was used in addition to the EB welding method described above. Autogenous welds were created by a Hobart Cybertig 500-DCs power source using the parameters given in Table 3 . Shielding gases with compositions of 1008 Argon or 758 Helium plus 258 Argon were used. The mixed shielding gas with the ratio noted was expected to produce a more uniform weld with a higher depth-to-width ratio. Rotational GTA welds in which the sample was rotated during heating were also made. A variable-speed motor rotated the sample at approximately 


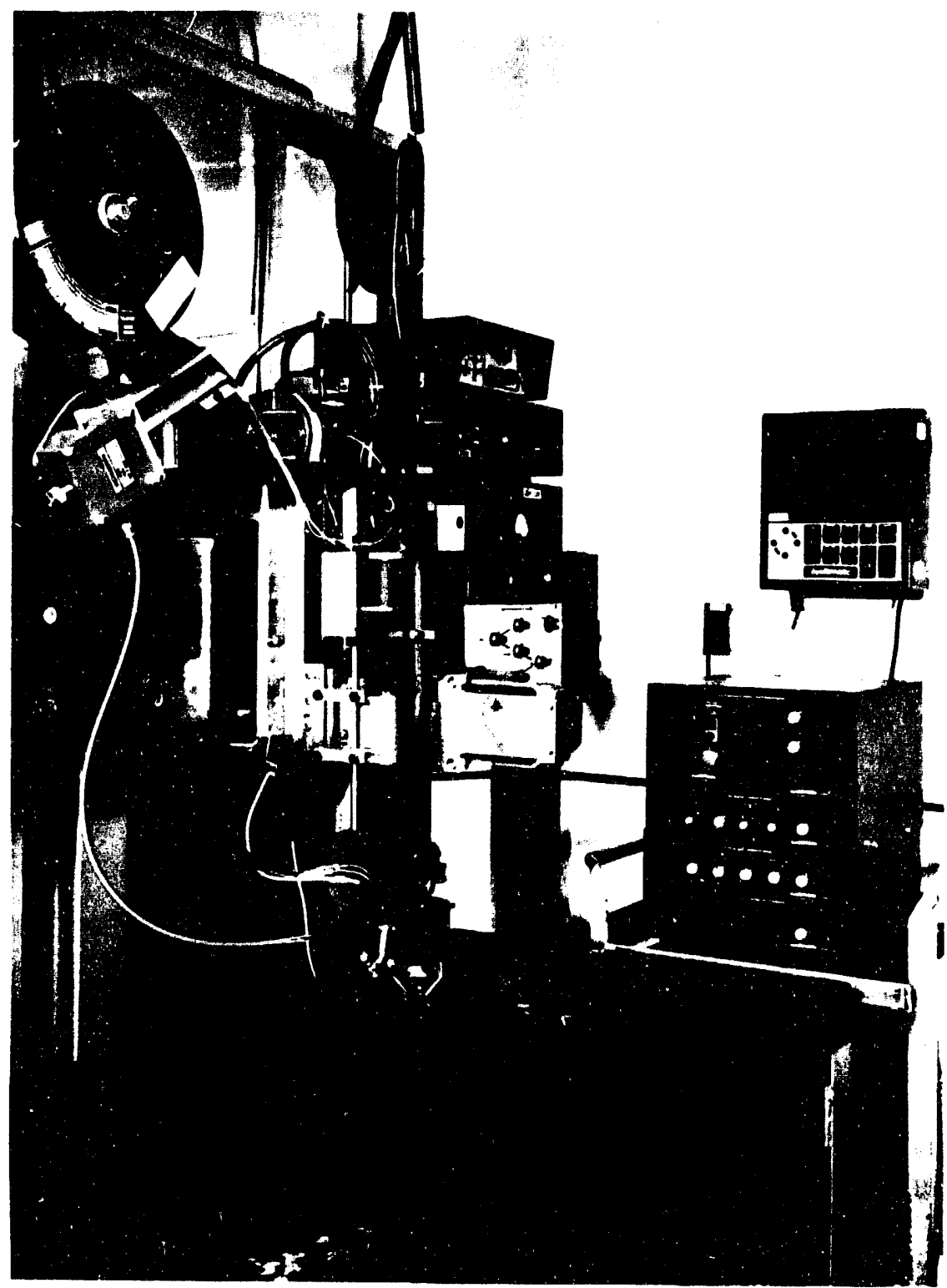

Figure 11

Gas Tungsten Arc Welding System 
Table 3

Gas Tungsten Arc Welding Parameters

Gas Tungsten Arc Welding Paremelers

Electrode

Electrode Angle

Eloctrode Extension

Electrode Stick-ouk

Polerity

Arc Ingrition

Gas Preflow Time

Automstic Upslope Time

Weld Taper Time

Automatic Dounstope Time

Gos Postlfow

Ges Flow Rate

$100 \%$ Argon 850 abic meters per hour

75\% Heium 25\% Argon 1400 cubic meters per hour

1 revolution-per-second during the formation of the melt pool.

The rotation of the sample during welding was carried out with the goal of producing a more uniformly circular melt pool by an effective thermal averaging of the heat supplied to the sample surface. The rotational welds did appear more uniform in regard to the shape and contour of the weld-pool as compared to the 
stationary welds. However, for each of the processes (i.e., rotational and stationary), the resultant microstructure was predominantly the same.

\section{Netallographic Procedurea}

\section{Sectioning of the Welded Crystal}

The microstructural features of the welds were characterized by standard metallographic procedures. The singlecrystal welds were sectioned at various angles predetermined by using the Laue back-reflection $x$-ray-diffraction technique (Figure 12). A Laser Technology, Lastec Wire Saw with a cutting mixture consisting of Silicon Carbide Powder and Glycol and with a tungsten wire whose diameter was $0.02 \mathrm{~cm}$ was used to section the weld at the desired orientation. Each cut was made so that the edge of the wire was approximately $0.01 \mathrm{~cm}$ from the center or the edge of the weld to correct for surface loss during the initial polishing that was required in order to obtain the desired metallographic information from the weld.

Sample Mounting, Polishing, and Etching

The samples were mounted in an epoxy mold using Epodyne resin and hardener and Buehler silicone mold release. Grinding 


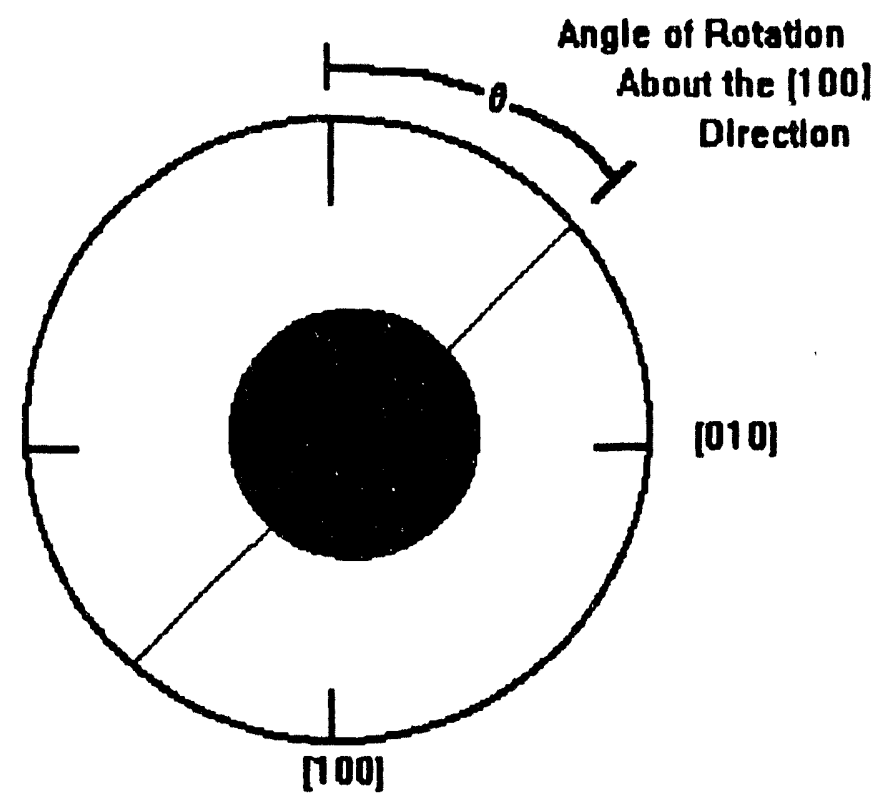

Figure 12

Sectioning Configuration for Angled Cuts

of the sample was performed using a Buehler Polimet I Polisher and Buehler sandpaper. The polishing of the crystals was divided into three phases. The first phase involved a syntron Laboratory Feeder using Buehler Micropolish (Alpha Alumina 3 Micron) and distilled water on a Buehler Texmet Polishing Cloth. The second polishing phase used an Elgin Dymo 0.5 micron diamond paste and Ethylene Glycol on a Buehler Nylon Polishing Cloth. A final polish using silica and distilled water on a Buehler Mastertex Polishing Cloth was performed to obtain a high-quality finish on the sample surface. For examinations of the microstructure by 
optical microscopy, the etchant employed was a 108 Oxalic Acid solution. The electropolishing was accomplished with a TCR10 power source set on a voltage control of 3.5 volts with a current ranging from 0.3 to 0.6 amps for approximately 45 seconds.

\section{Photography}

Optical-metallographic photography was carried out by using a Bausch L Lomb Regearch II Metallograph, a Reichert-Jung MeF3 camera, or in the case of polarized-light photographs, a Cannon 35 mam camera attached to another optical metallograph.

\section{Milorohardinese}

Microhardness measurements were made to determine if a hardening effect occurred along the slip lines created by the welding process. These measurements were obtained using a diamond pyramid indenter on a Shimadzu Microhardness tester with a load of 200 grams and a test time of 15 seconds. For each hardness measurement, the diameter of the hardness indent was measured in two directions and then averaged. This average was then used in conjunction with the Shimadzu Handbook for Microvicker Hardness Numbers to determine the hardness of the crystal at that particular point. These hardness values were calculated using the formula: 


$$
\text { Hardness }-1854.4\left(P / d^{2}\right)
$$

where:

P was load in grams

d was average diagonal diameter of the indented diamond. The hardness measurements were performed at intervals along the slip lines in the [100], [110], [010] directions, in so far as it was possible without impinging on the other welds or on the edge of the crystal. Measurements on an unwelded crystal were also made to determine the extent of hardening of the welded crystal.

\section{Profilomatry}

In order to determine the nature of the variations (1.e., frequency and amplitude) of the ripples observed on the surface of the weld, a topographic analysis of the weld was performed. A Taylor-Hobson Talysurf 10 microhardness instrument with a diamond tip and a Televideo PC with TS10 version 4.5 software and an Epson EX-850 printer were used to perform the topographic mapping of the samples. The diamond point measured eight cutoff lengths (0.25 millimeters each) to obtain a surface profile of the weld from the edge of the weld to approximately its center. Each cutoff length determined the individual data sampling area. From this data collection, graphical-analysis software was used to obtain a profile, distance of scan, and overall amplitude from 
the surface being tested. This analysis also provided information that determined the amplitude, frequency, and spacing of the observed surface ripples on the weld.

\section{8umary}

The procedures described above for the crystal growth, welding, metallographic analysis, microhardness, and profilometry experiments indicate how the present data were obtained, characterized, and analyzed. Using these procedures it was possible to determine the resultant microstructure for a singlecrystal spot weld and to determine the dendritic-growth pattern for the three planes, (100), (110), and (111). Each of the welding methods used in the microstructural investigation produced a unigue weld-pool shape, resulting in distinct microstructural and solidification patterns for each method. The experimental methodology described here also led to the determination of other valuable physical parameters such as the microhardness and surface profiles of the single-crystal welds. The quantitative metallographic data were subsequently used to carry out a reconstruction of the resulting microstructure. The results to be described in the following section represent the basis for the future development of a mathematical analysis that can provide the capability for predicting the microstructure of a 
single-crystal stainless-steel spot weld for weld pools with a given shape. 


\section{CEAPIIHR 4}

\section{RESULTS AND DISCUSSION}

In this section, the solidification microstructures resulting from the formation of stationary melt pools on oriented surfaces of stainless steel single crystals are examined. Solidification microstructures were formed in stationary melt pools (1.e., pools formed with the heat source fixed) on the three oriented single-crystal surfaces: (001), (011), and (111). The microstructural results allowed for a reconstruction of the solidification characteristics of the melt pools in three dimensions. The mechanical properties of the heat-affected zone near the solidified melt pool were examined using microhardnessIndentation techniques, and surface profilometry was applied to characterize the oscillatory surface structures observed on the solidified pool surfaces. The results of these investigations are treated on a case-by-case basis in the following sections. 


\section{Marostructural Analyais cas Iungsten sro Felding 750 Eelium and 258 Argon}

\section{1) Crystallographic Orientation}

A (001) stereographic projection for a cubic-crystal system is shown in Figure 13 [33]. As can be seen from this profection, in the plane perpendicular to the (001) axis there are twoadditional <100>-type axes and two <110>-type axes located $45^{\circ}$ away from the $\langle 100\rangle$ axes [34]. The $\langle 100\rangle-t y p e$ axes have been determined as the preferred dendritic growth directions and are noted in rectangular areas.

GTA heating was used to form partial penetration stationary melt pools. The microstructural investigation on the (001) surface utilized both cross-section micrographs along principal directions, as well as information obtained by progressively grinding away the top surface of a (001) surface weld to reveal the microstructure at various depths into the weld. A combination of the cross-sectional and surface "in-depth" views allowed for a reconstruction of the dendrite-solidification pattern in three dimensions.

Sectioning Along the $[100]$ Direction: A schematic for the melting configuration is represented in Figure 14. As illustrated in the schematic, the heat source was ideally located 


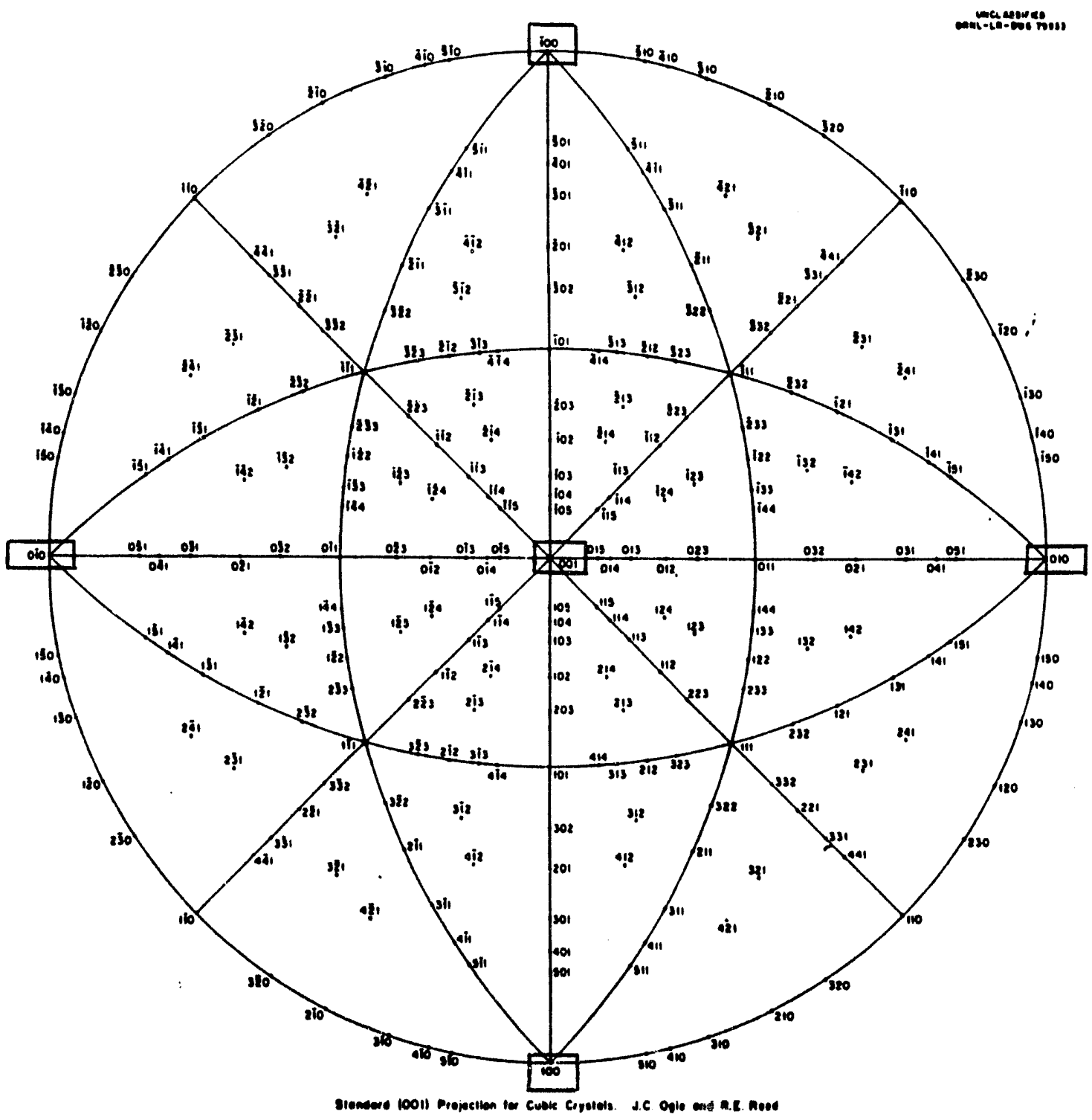

Figure 13

Stereographic Projection for the (001) Plane 


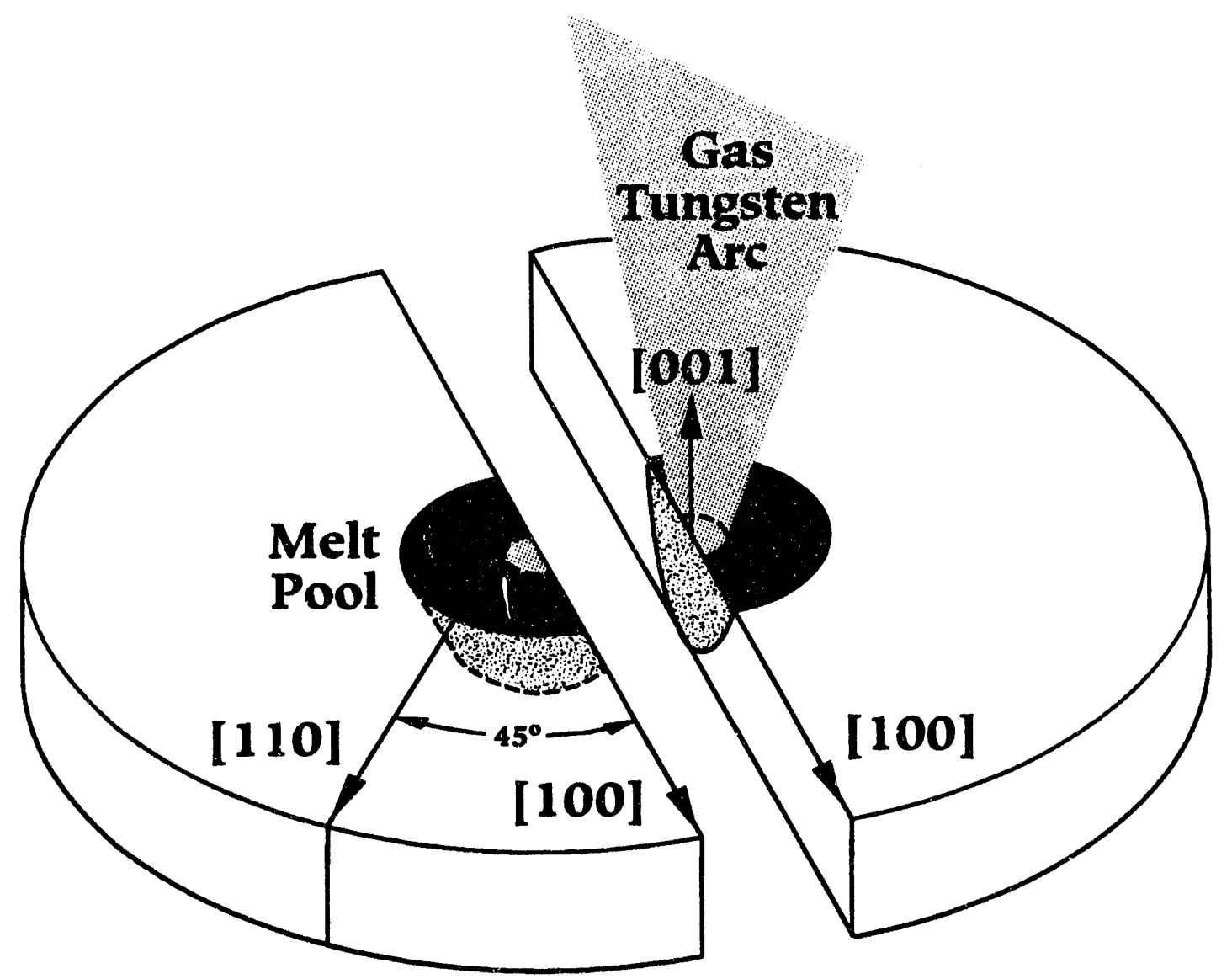

Stainless Steel Single Crystal (001) Surface

\author{
Figure 14 \\ Schematic for (001) Surface [100] Direction \\ Sectioning and Welding Configuration
}


at the center of the single-crystal or, for the case of multiple melt pools, formed on one crystal as far from the edge as possible. This figure also illustrates the sectioning of the crystal to obtain cross-section micrographs which were parallel to the [100] direction of the melt pools.

Figure 15 represents the (001) top surface macrograph along with the cross-sectional view along the [100] direction. The schematic in this figure represents a three-dimensional reconstruction of the resultant microstructure produced from the complete set of metallographic data obtained from these views, i.e., the in-depth top view, and the [110] cross-sectional view.

Figure 15b shows the top-surface dendritic growth proceeding from the perimeter of the weld toward the center in the [100] directions. The central portion of the weld shows the crosssections of the [001] dendritic growth. This can be better visualized in the cross-sectional view, Figure $15 \mathrm{c}$, in which the [001] dendrites are clearly seen. It can also be seen in this view of the [100] dendrite-growth pattern. These views are illustrated in the three-dimensional reconstruction shown in Figure 15a. The reconstruction, Figure 15a, illustrates the microstructurally determined solidification process for the melt pool. Noted on this figure are the angles in which the dendritic growth occurred. The dendrite directions are illustrated by the sprocketed cylinders. The melted surface solidified from the 


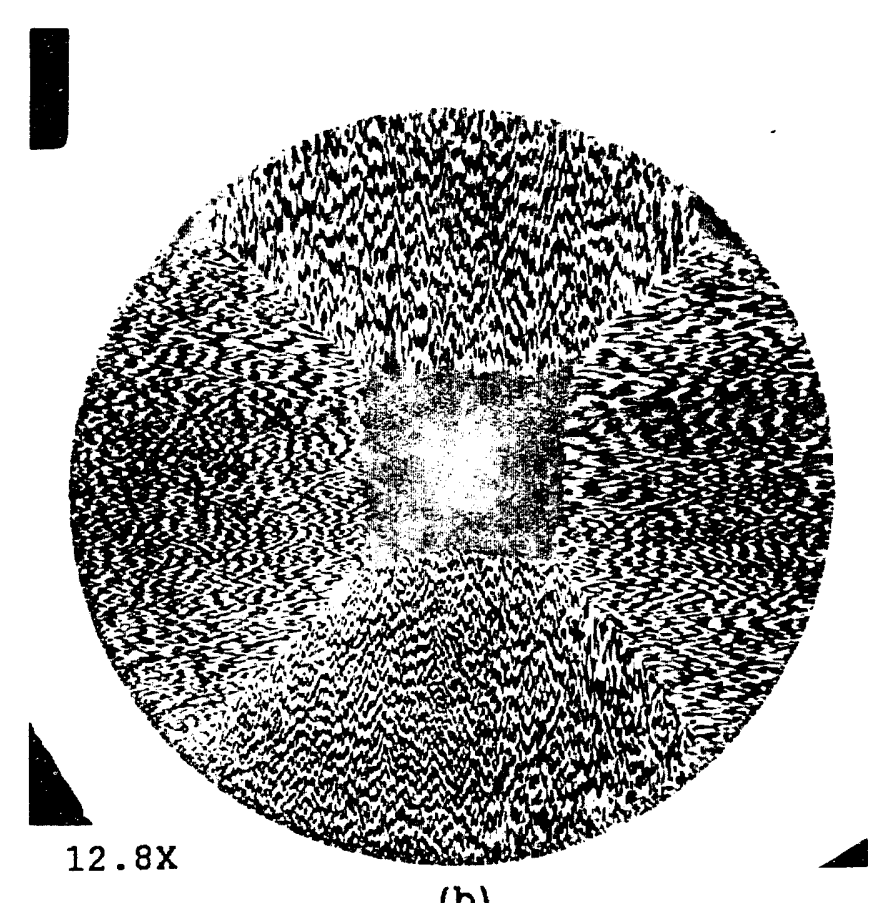

(b)

(001) Surface View GTA Stationary Top of Weld

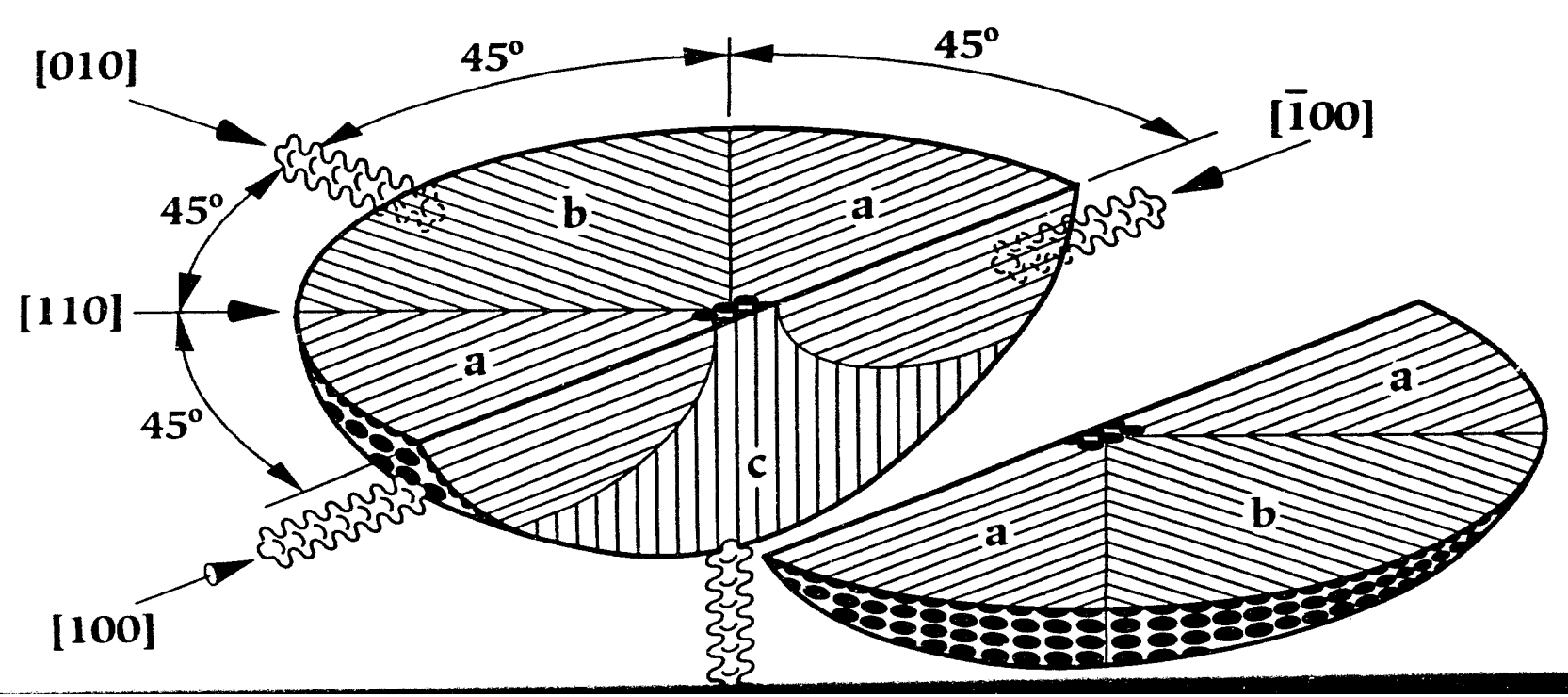


(001) Surface

Gas Tungsten Arc Weld

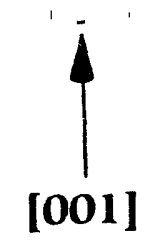

(a)

Three-dimensional Reconstruction of the Resultant Microstructure

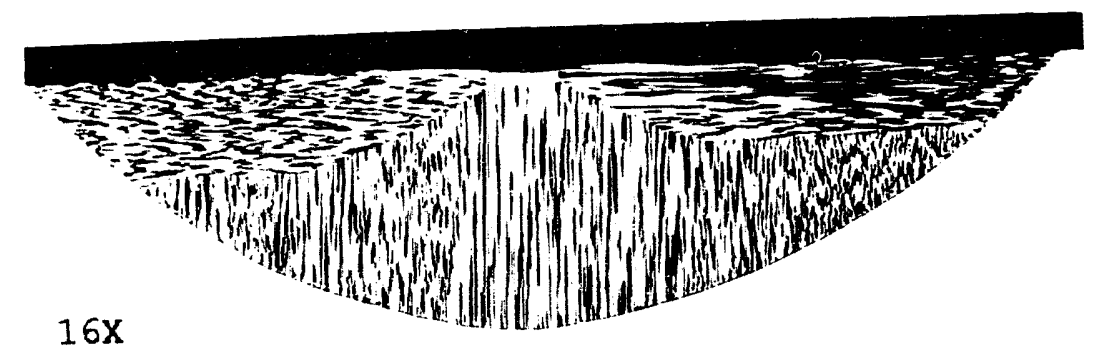

(c)

Transverse View GTA stationary Weld [100] Direction

Figure 15

Three-dimensional Reconstruction of (001) Plane [100] Direction

Stationary GTA Weld With Accompanying Photomacrographs 
edge of the weld inward, along the [100] directions in "a" areas and in the [010] directions in "b" areas. In area " $c$ " solidification proceeded from the bottom and the sides of the weld along the [001] direction. The four-fold symetry of the microstructural pattern clearly reflects the four-fold symetry of the (001) plane.

Rectangular areas "A", "B", and "C" in Figure 16 denote areas for further microstructural analysis. These areas also aid in determinations of the details of the solidification process of the melt pool. Area "A" of Figure 16 is represented in Figure 17. This microstructure demonstrates a solidification pattern closer to the center of the weld. Note that as the center is approached, the [001] dendrites increase in length before meeting the [100] dendrites causing an upward curve to the boundary representing the union of the [001] and [100] dendrites. This is because the dendrite velocities are different for the [001] and [100] directions. The velocity varies because there is a variation in the angle between the dendrite growth and the solidification front between the two directions.

Figure 18 represents the microstructure of area "B" in Figure 16. This area shows the solidification intersection of the [100] and [001] dendrites near the edge of the melt pool. It can be seen that the [001] dendrites terminate along the long axis of the [100] dendritic cells indicating that they had 


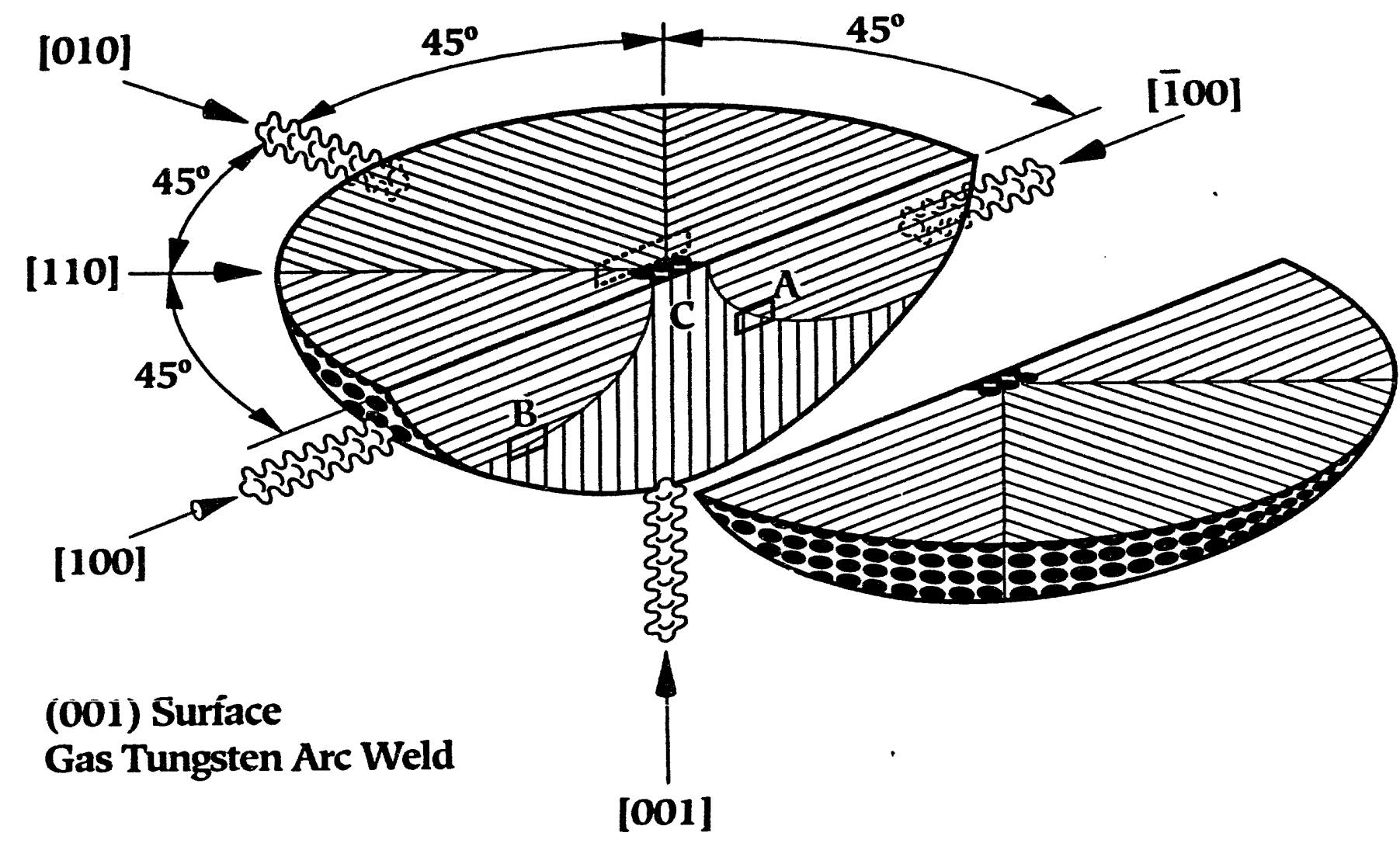

Figure 16

Three-Dimensional Reconstruction Identifying 


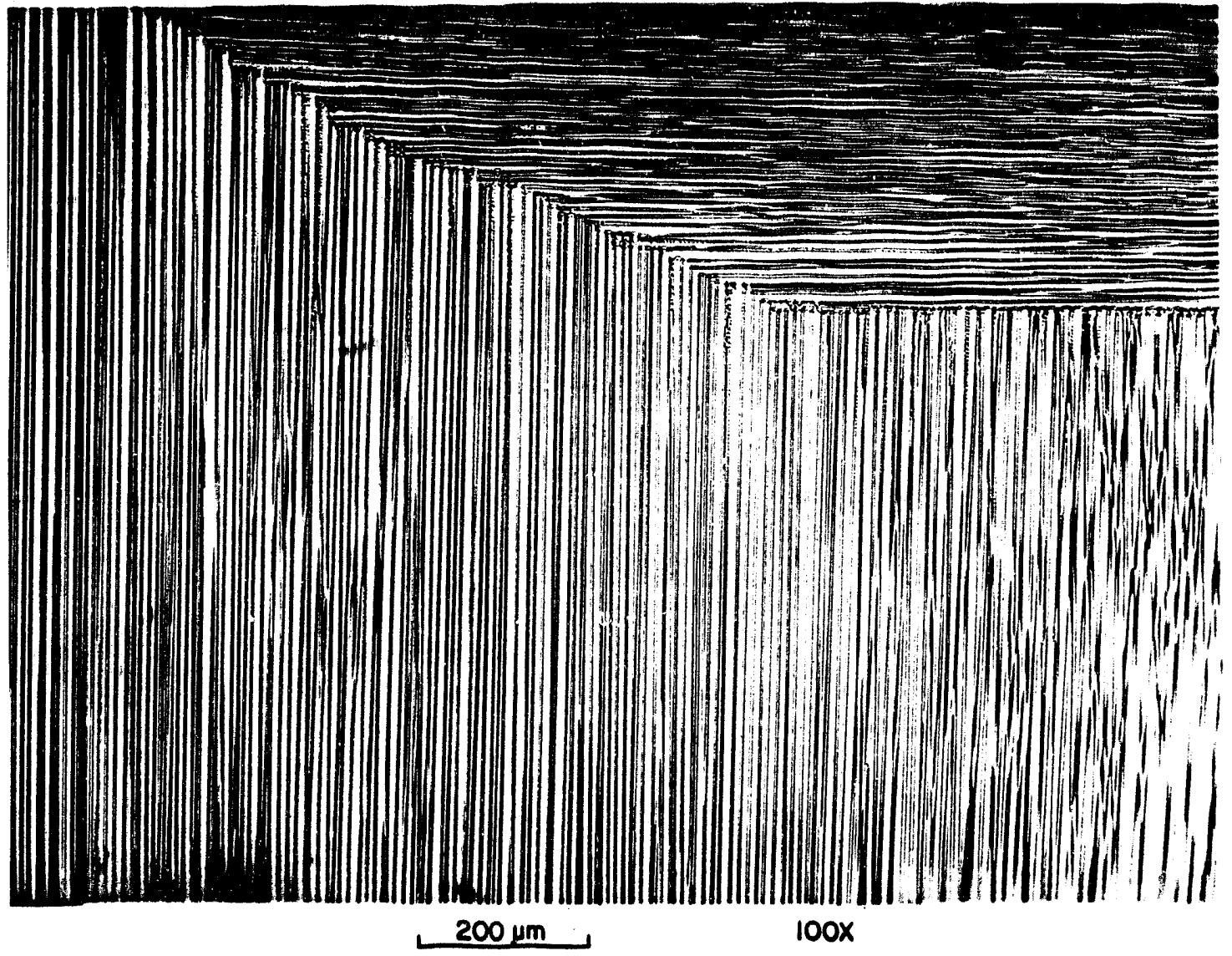

Figure 17

[100] Transverse View of stationary GIA Weld

Dendritic Growth in the Weld

(Area "A" of Figure 16) 


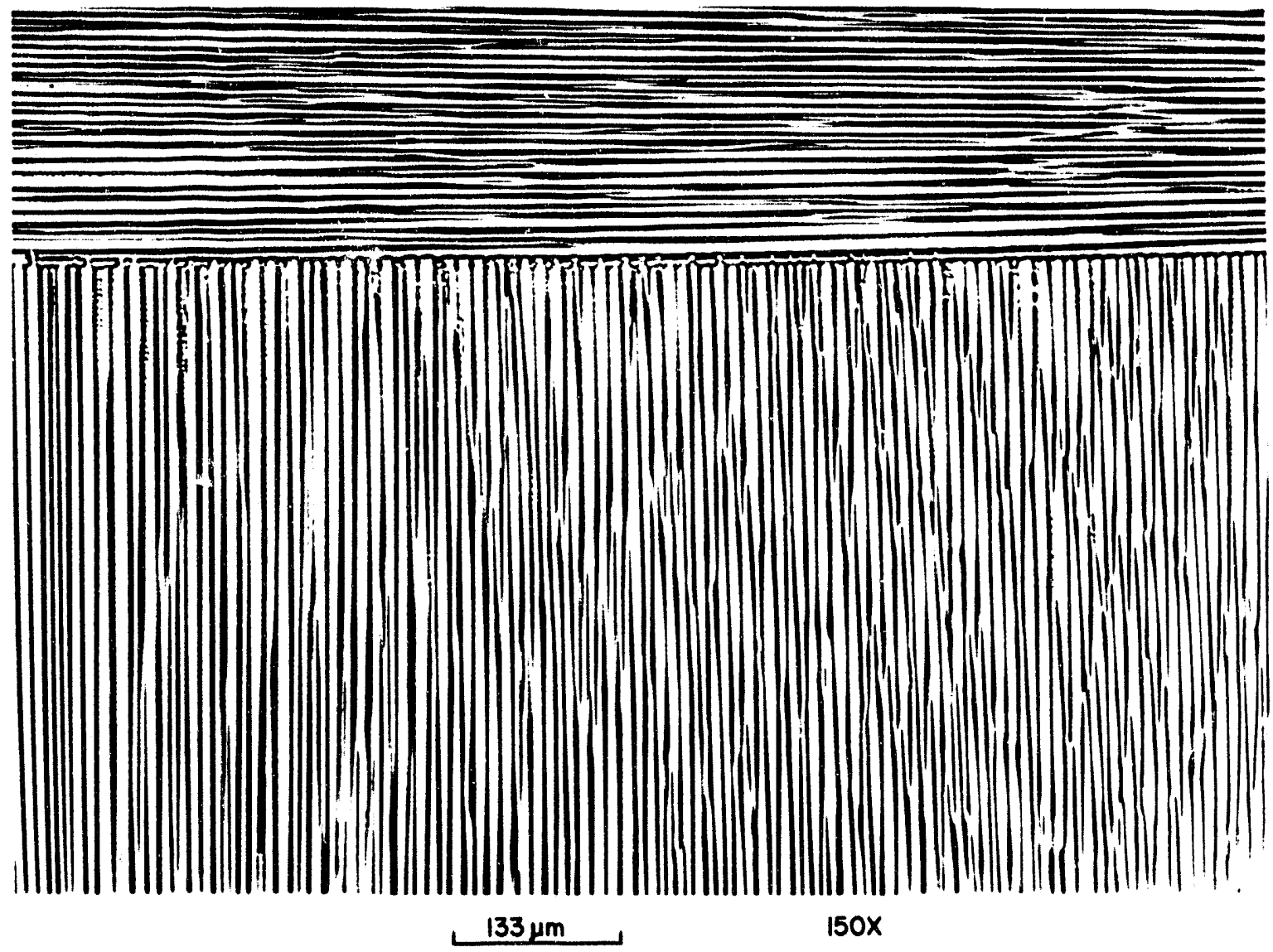

[100] Direction

Figure 18

[100] Transverse View of stationary GTA Weld

Solidification phenomena

(Area "B" of Figure 16) 
solidified prior to the solidification front of the [001] dendritic growth.

The dendritic growth and weld symmetry of area "C" in Figure 16 can be seen microstructurally in Figure 19. This figure represents the microstructure of the [100] transverse view past the center of the weld. The [010] dendrites form a "square section" at the top of the micrograph and upward arc of the [100] dendrites and the $[001]$ dendrites as illustrated in Figure 17. The weld symetry of Figure 19 shows that one dendritic growth direction does not dominate any of the other preferred growth directions and that the growth rates were approximately the same in each respective half.

Top Surface: Top surface views were achieved by using one weld and progressively grinding through the weld thereby obtaining the solidification microstructure at various depths in the weld. The welding configuration for the surface welds appears schematically in Figure 20. This schematic 1llustrates the method of forming the melt pool as well as the primary directions. Figure 21 depicts the three-dimensional reconstruction and the accompanying set of surface views which were used to create the reconstruction. The top-surface photomacrograph in Figure 21b clearly shows a four-fold symmetry in the weld microstructure from the [100] and [010] dendrite 


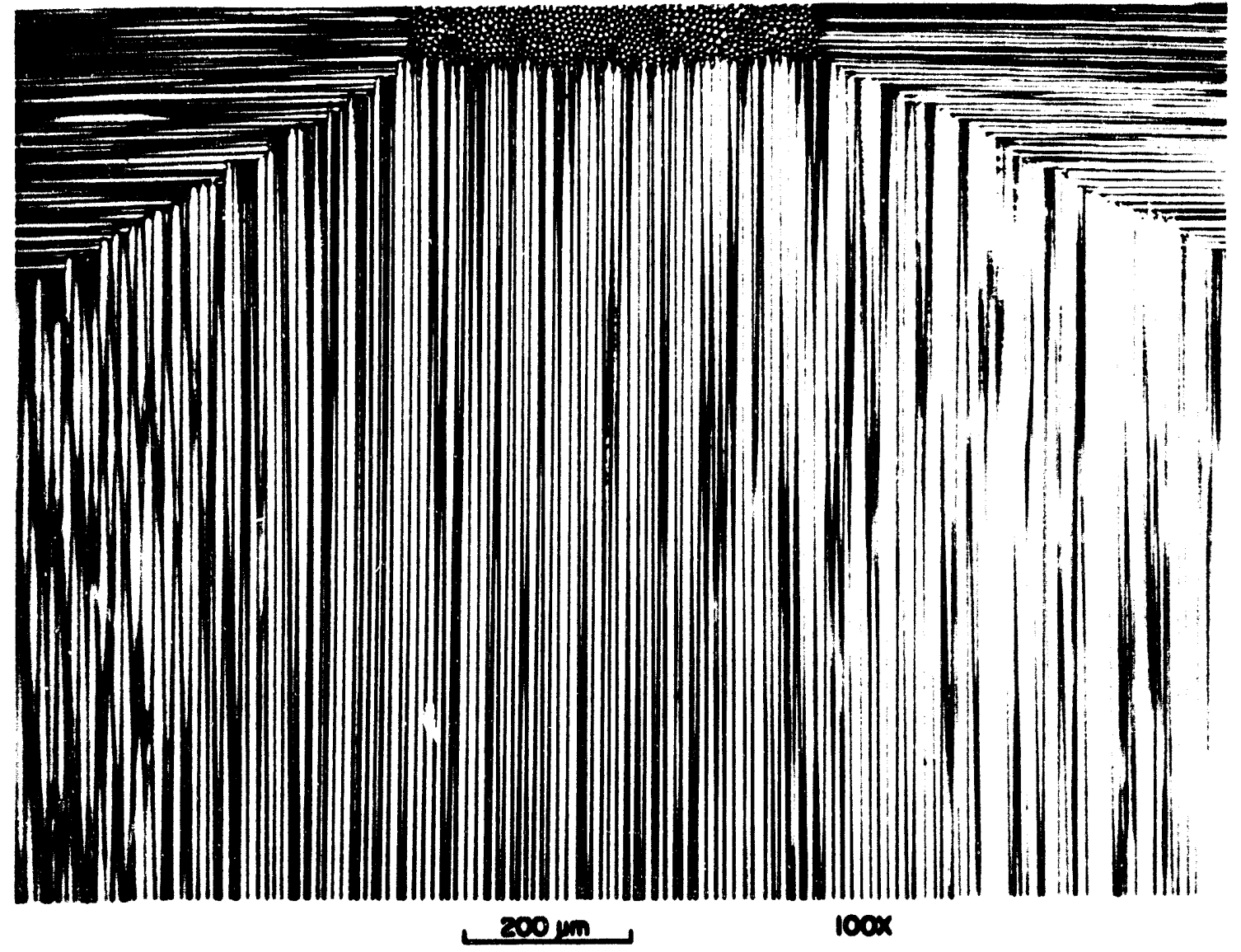

Figure 19

Center Section of [100] Transverse View

(Area "C" of Figure 16) 


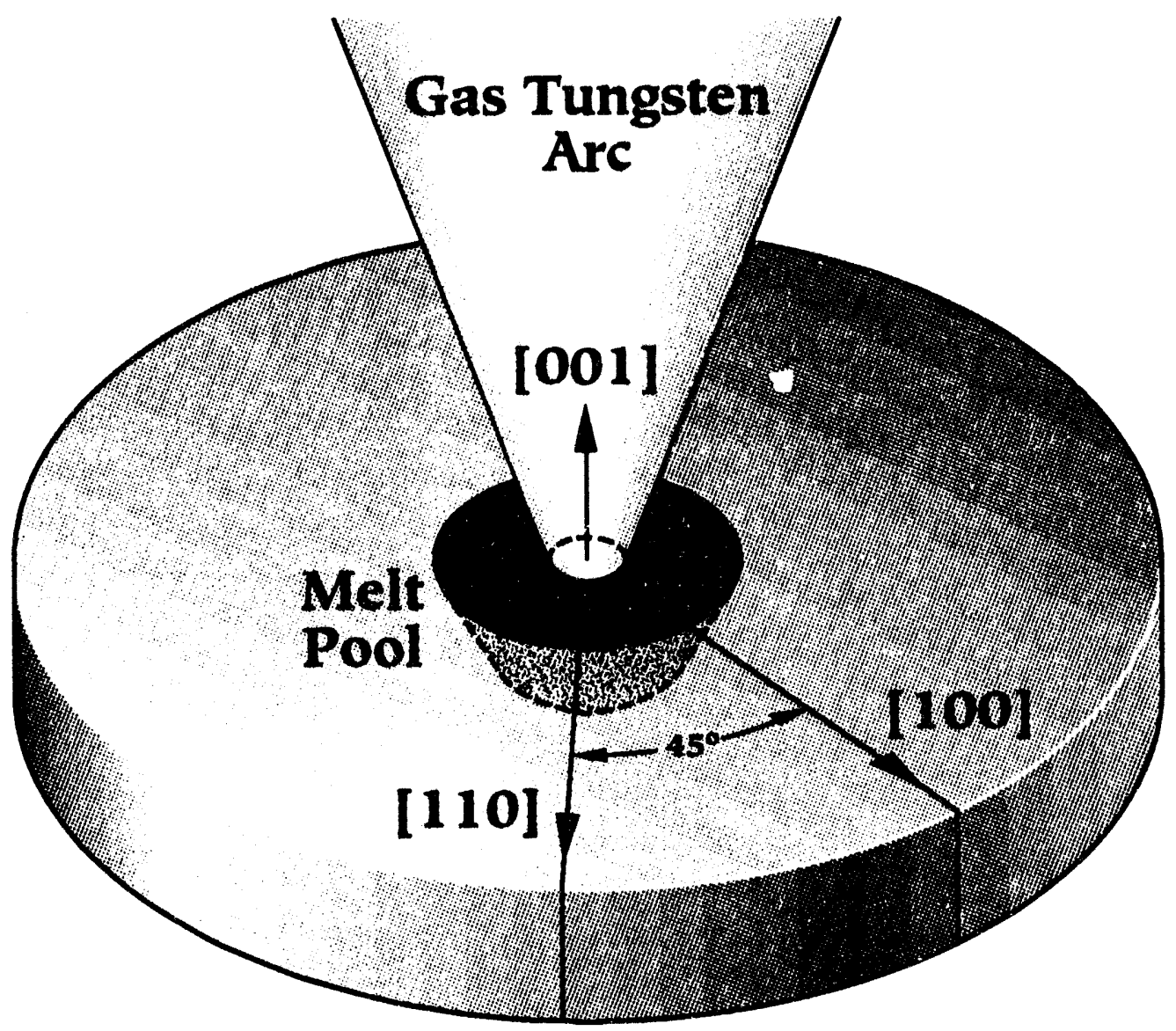

Stainless Steel Single Crystal (001) Surface

Figure 20

Schematic for (001) Surface welding Configuration 


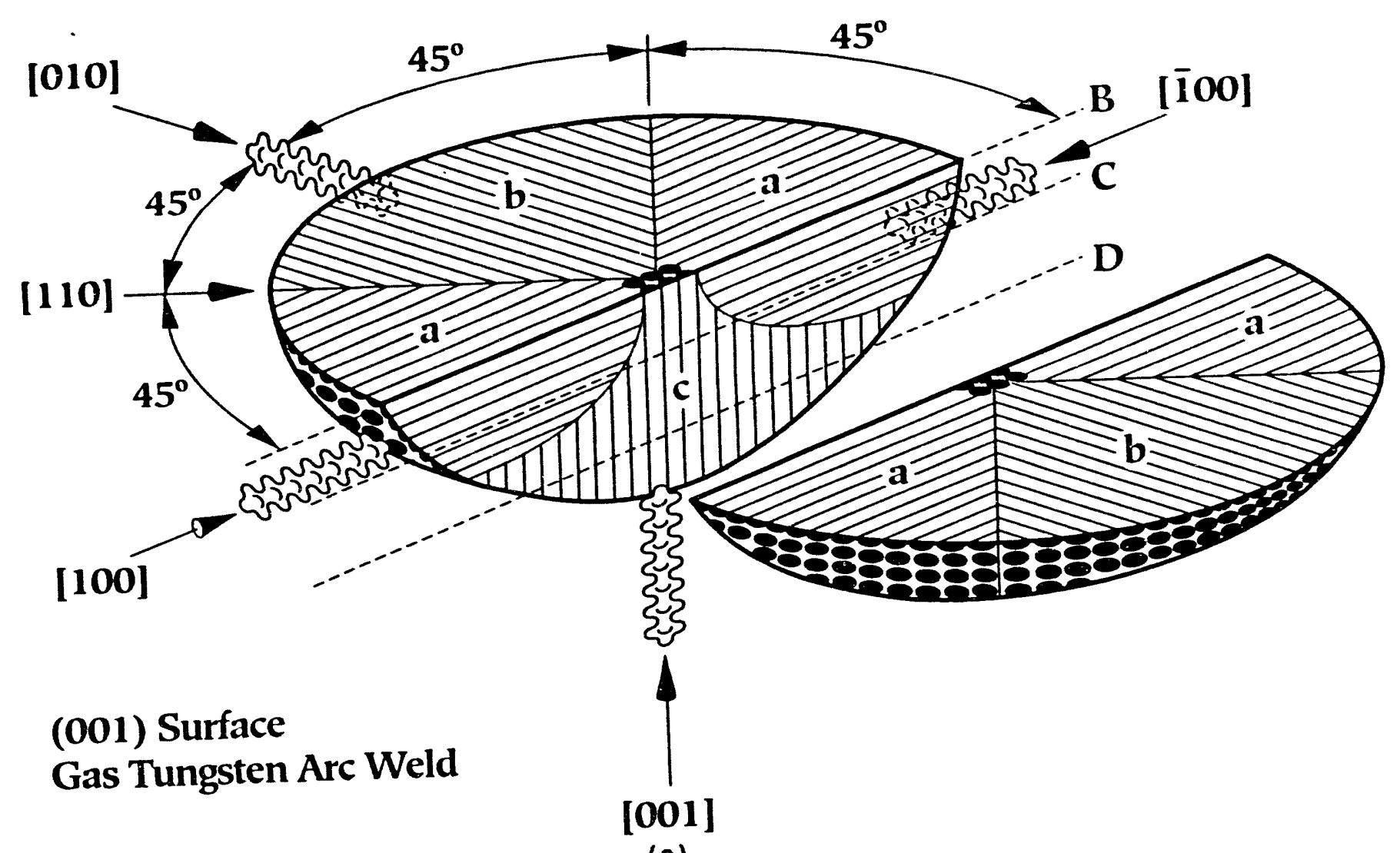

(a)

Three-dimensional Reconstruction of (001) Surface

stationary GTA Weld 


\section{(b)}

(001) Surface View GTA Stationary Top of weld

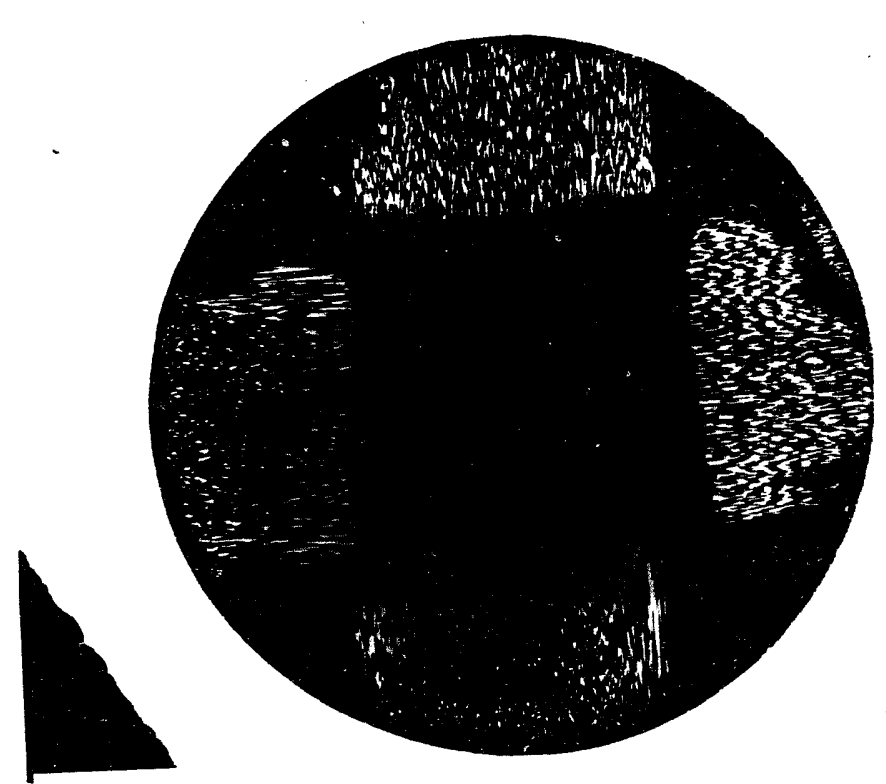

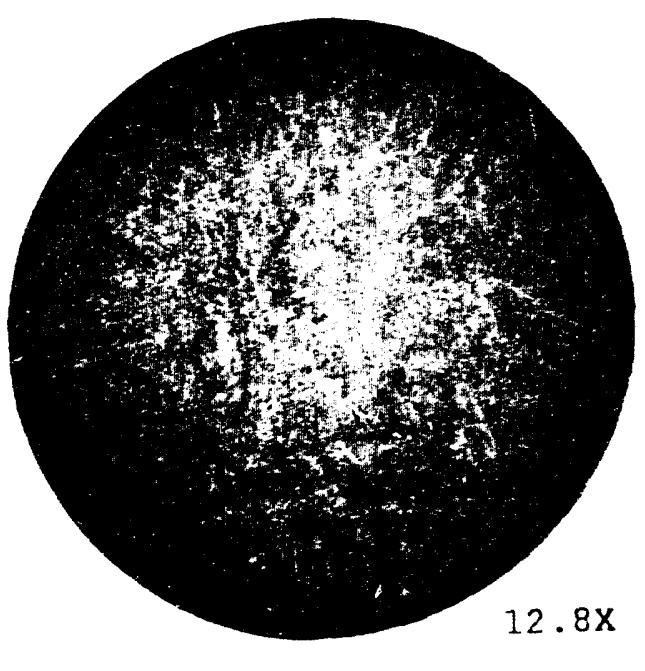

(d) (c)

$12.8 \mathrm{x}$

(001) Surface View GTA Stationary Weld

Deeper into weld

\section{Figure 21}

(001) Surface View GTA Stationary weld Complete Coverage of [001] Dendrites Three-Dimensional Reconstruction of (001) Plane Surface Stationary GTA Weld with Accompanying Photomacrographs 
growth directions and the center section with the [001] dendrites. The photomacrograph in Figure 21c that shows the concentration of [001] dendrites has increased at the center and illustrates the appearance of these dendrites on the edges of the melt pool. The incredible symetry of the system can also be seen in this particular top-surface view or manifested in the repetitiveness in each of the four quadrants. This effect is attributed to the uniform weld shape, the fact that each of the preferred growth directions are $90^{\circ}$ apart, and the comparatively symetrical growth rates in each of the four quadrants. Figure 21d shows a further increase in the [001] dendrites to completely cover the weld surface. These microstructures were used in creating the three-dimensi construction presented in Figure 21a. Noted on this figure. ne angles at which the dendritic growth occurred.

Figure 22 presents a schematic showing the sections of higher-magnification photomicrographs that were used to evaluate additional microstructural phenomena denoted by the rectangular areas " $A$ " and "B". Figure 23 microstructurally represents area "A" of Figure 22. This section provides evidence for the competitive aspect of dendritic growth in a $(001)$ surface weld. The pre-existence of dendrites, and the manner in which they grew, verifies that only one active growth direction is involved in any one area of the weld. This photomicrograph for the $(001)$ 


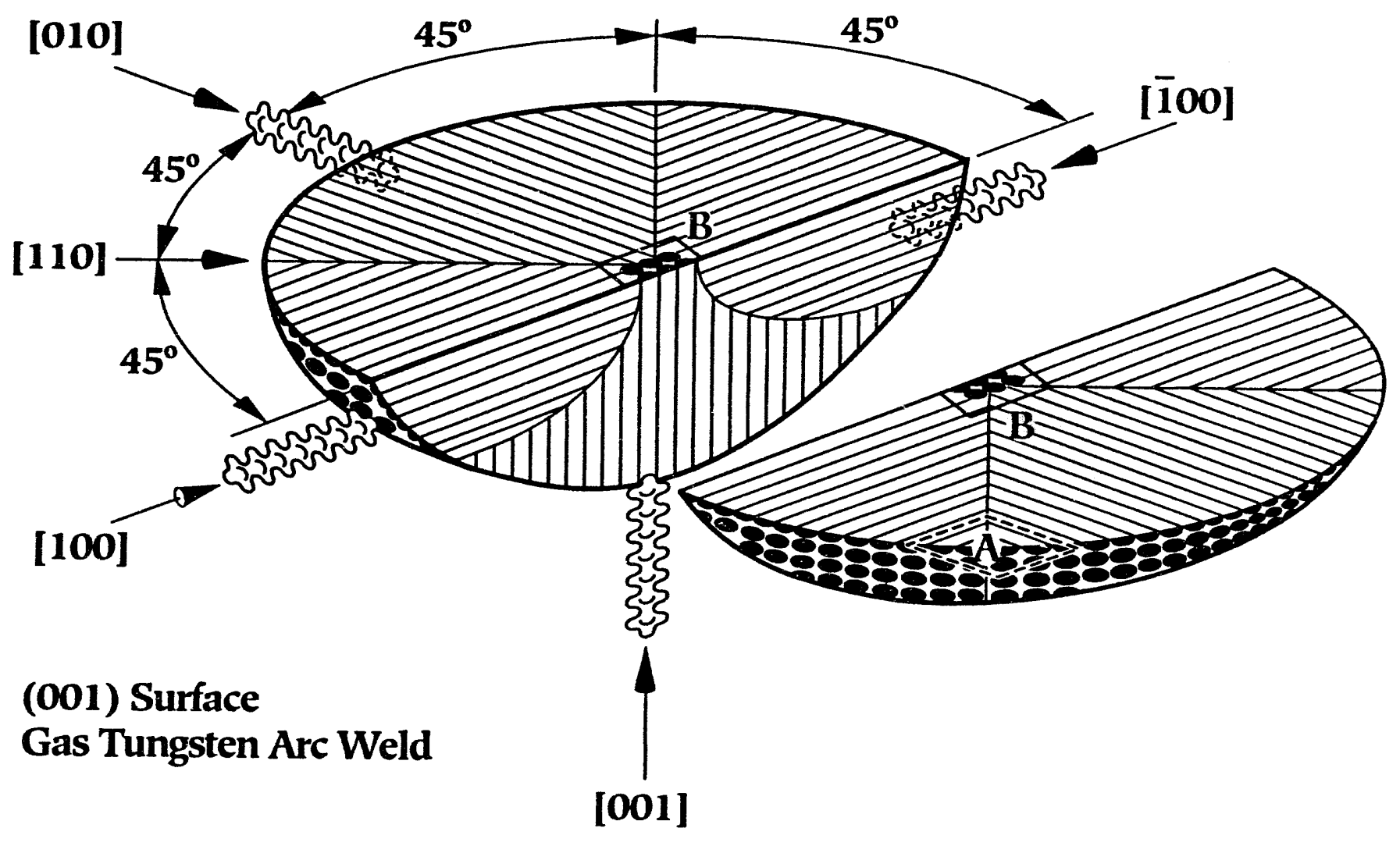

Figure 22

Three-Dimensional Reconstruction 


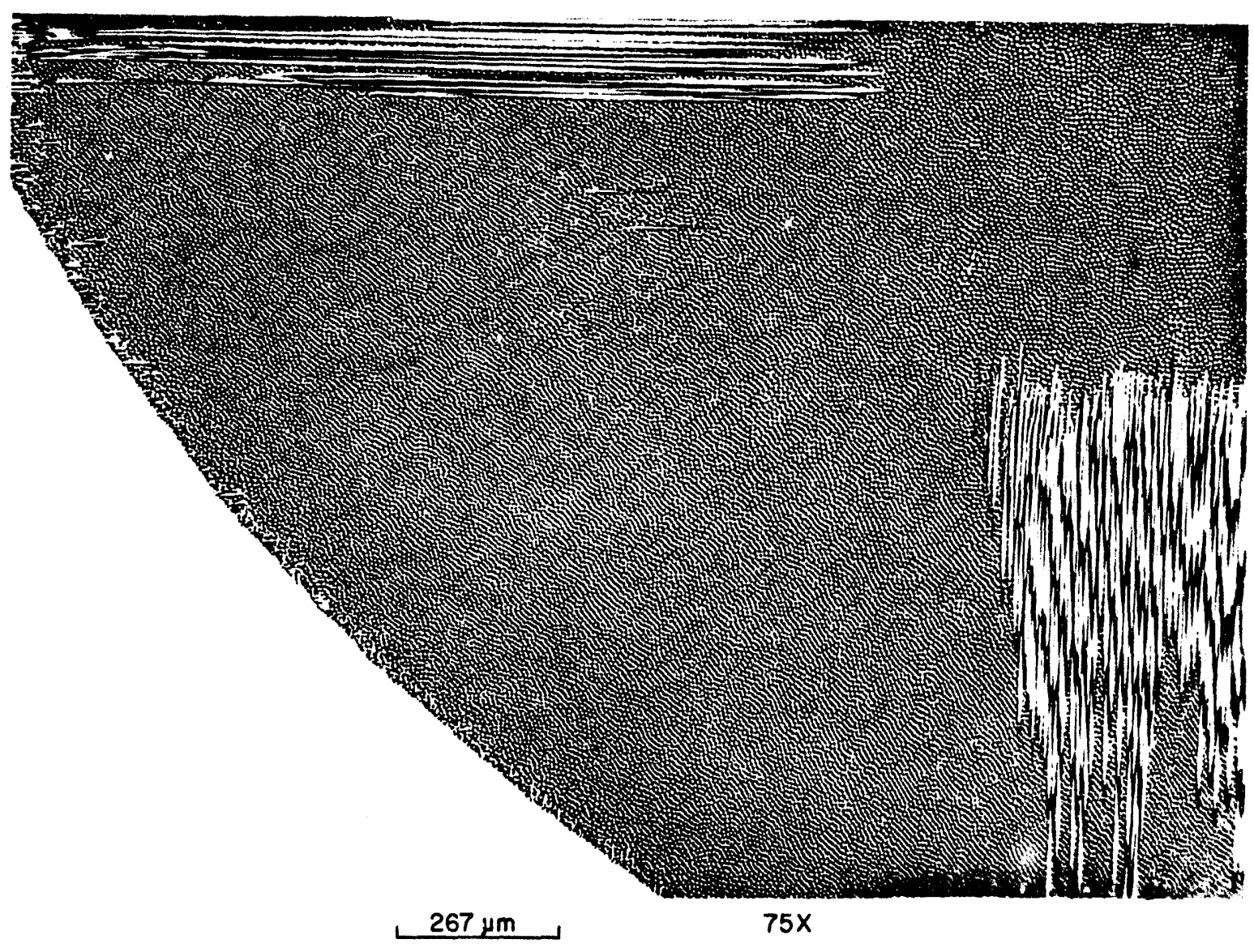

Figure 23

Lower Quadrant of (001) Surface of GTA Stationary Weld High Magnification Showing Competitive Growth

(Area "A" of Figure 22) 
plane illustrates that dendrites grew from the edges of the weld in the [100] direction and competed in direct competition with the [010] dendrites. Competition can also be seen in areas where the [010] dendrites grew around a [100] dendrite. The highermagnification photomicrograph of area " $B$ " shown in Figure 16 is presented in Figure 24. This photomicrograph clearly reveals the center-section weid symmetry and shows that dendritic growth along the [100] and [010] directions proceeded in each direction at comparable growth velocities.

Sectioning Along the [110] Direction: The welding and sectioning configuration for the [110] primary direction is represented schematically in Figure 25. This schematic illustrates the method of sectioning used to obtain the [110] cross-sectional view. Figure 26 presents the three-dimensional reconstruction with the accompanying photomacrographs. The [110] direction (Figure 26c) showed the same solidification features as in the [100] direction. Dendrites grew in the [001] direction from the bottom of the weld pool. But, the dendritic growth at the top of the melt pool appears elliptical cross-section in this view because the dendrites were growing at an angle of $45^{\circ}$ from the cross-sectional cut. Figure $26 \mathrm{~b}$ represents the top-surface view for the (001) plane, showing the same view as presented previously in which dendritic growth was from the edge to the center along the $[100]$ and $[010]$ directions and from the bottom 


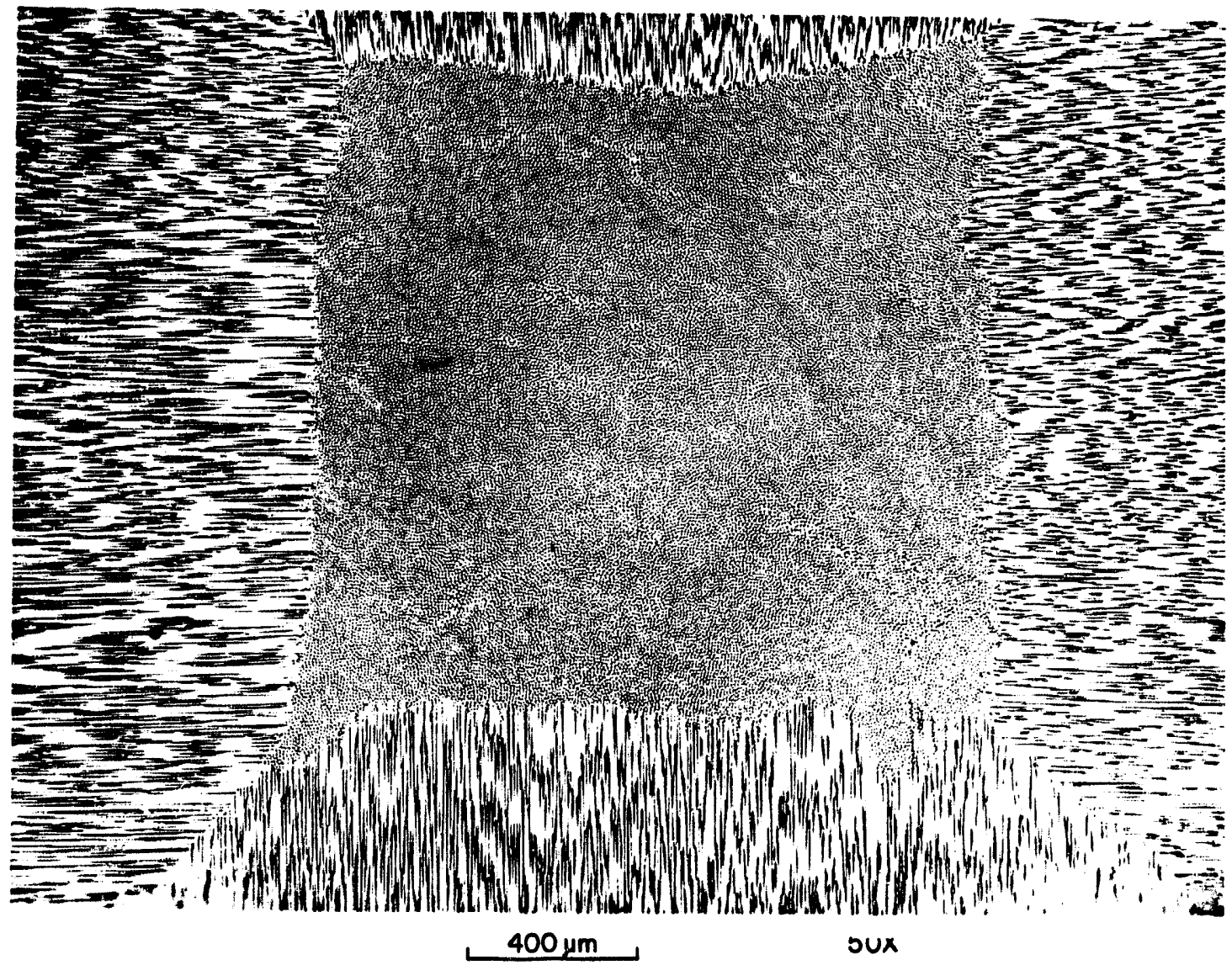

Figure 24

(001) Surface stationary GTA Weld

Center Section Symmetry

(Area "B" of Figure 22) 


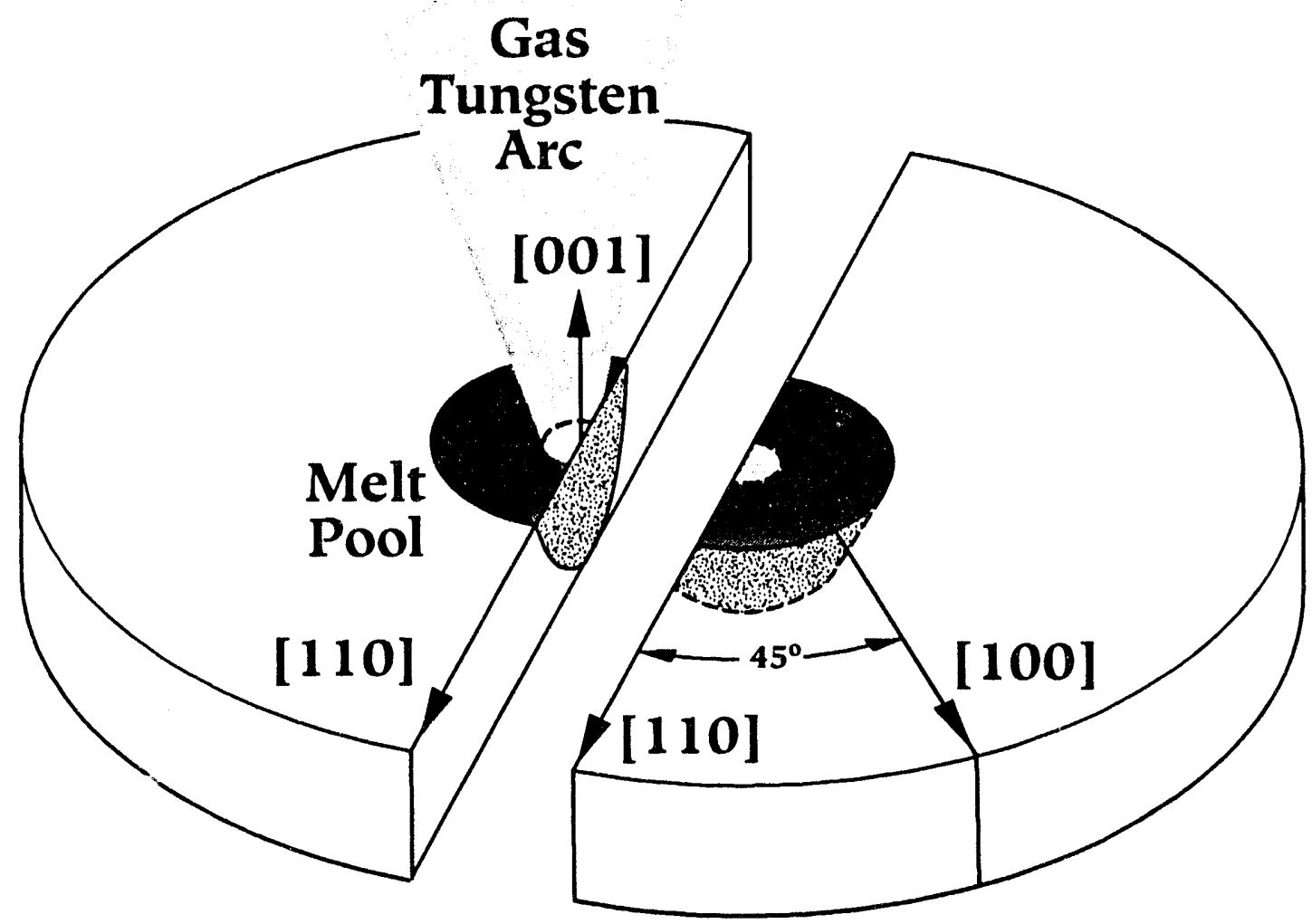

Stainless Steel Single Crystal (001) Surface

Figure 25

Schematic for (001) Surface [110] Direction

Sectioning and Welding Configuration 


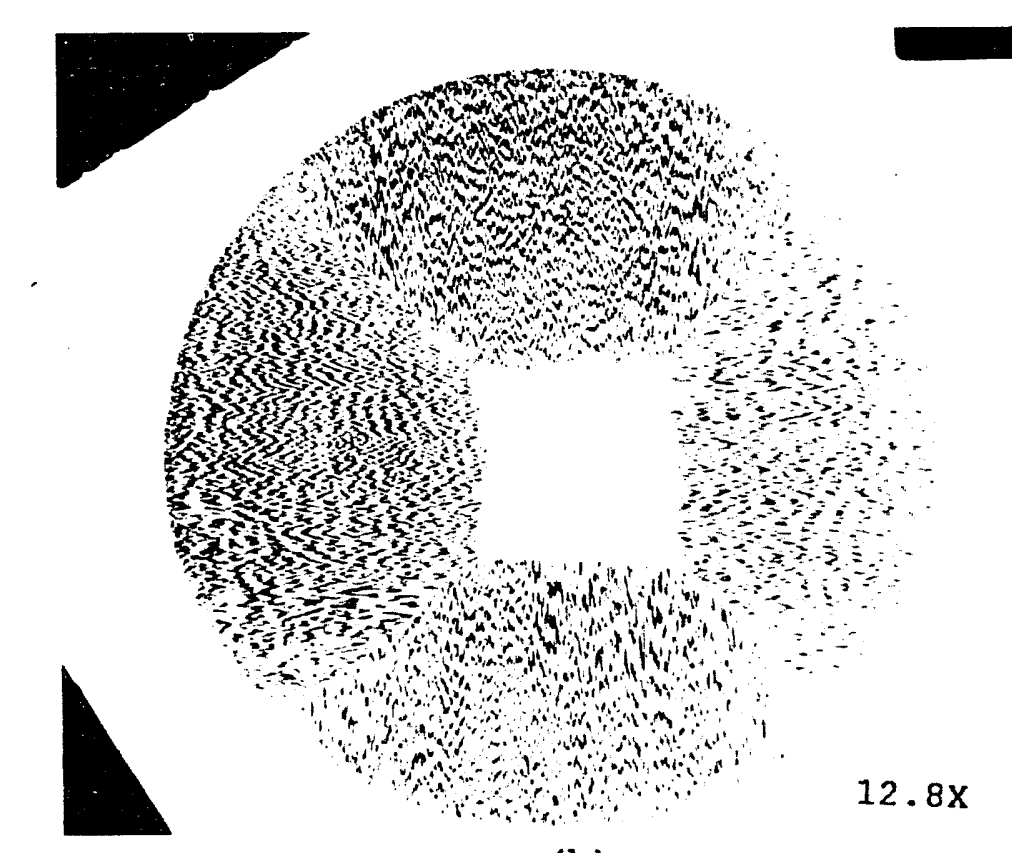

(b)

(001) Surface View GTA Stationary Top of Weld

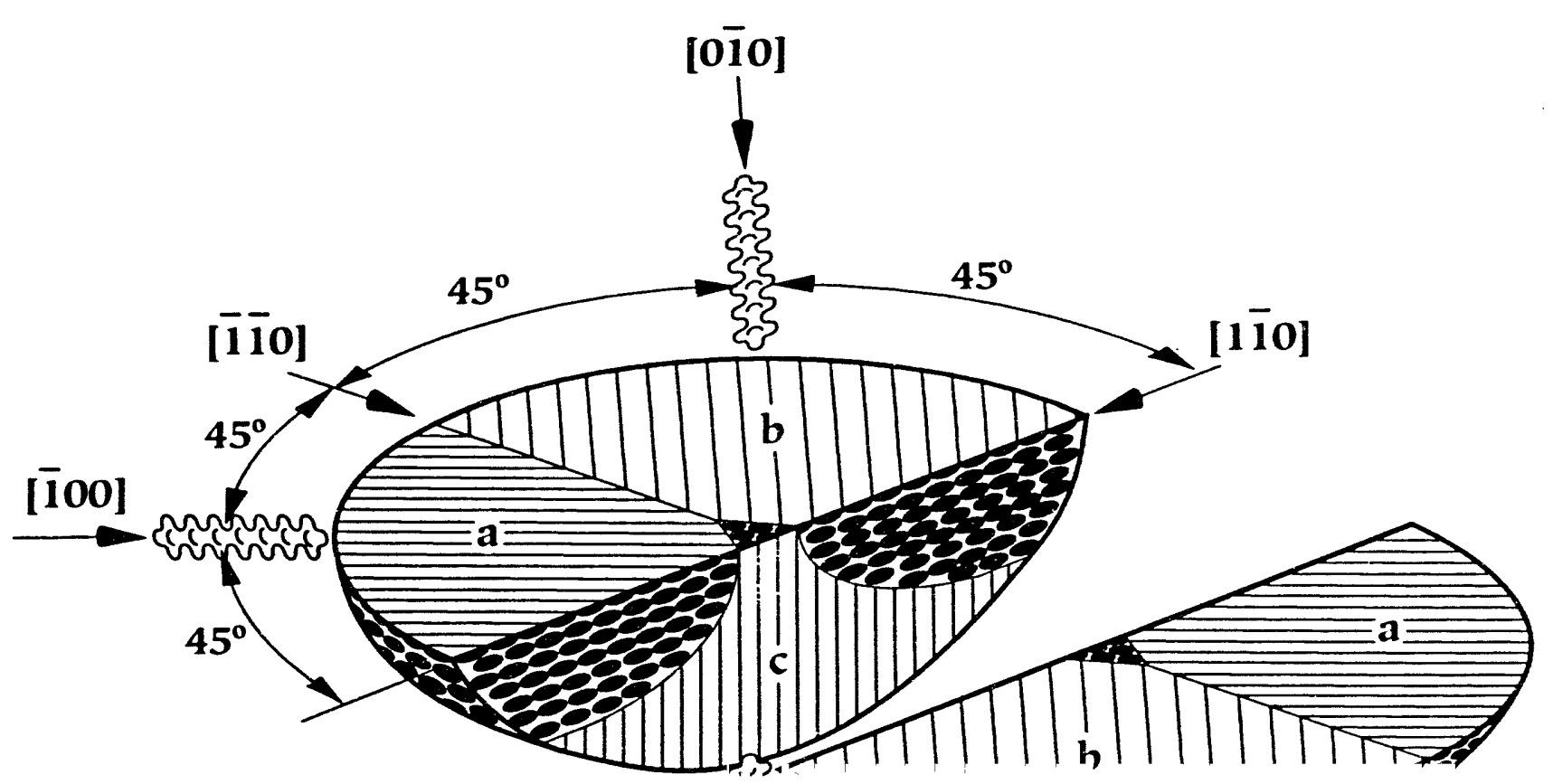


(001) Surface

Gas Tungsten Arc Weld

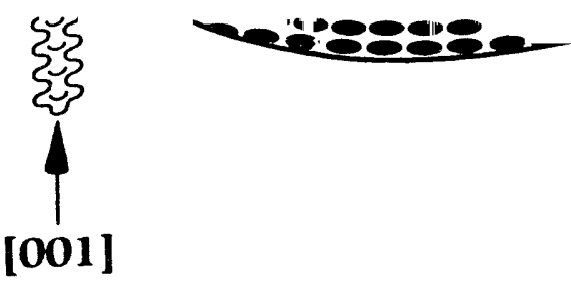

(a)

Three-dimensional Reconstruction of the Resultant Microstructure

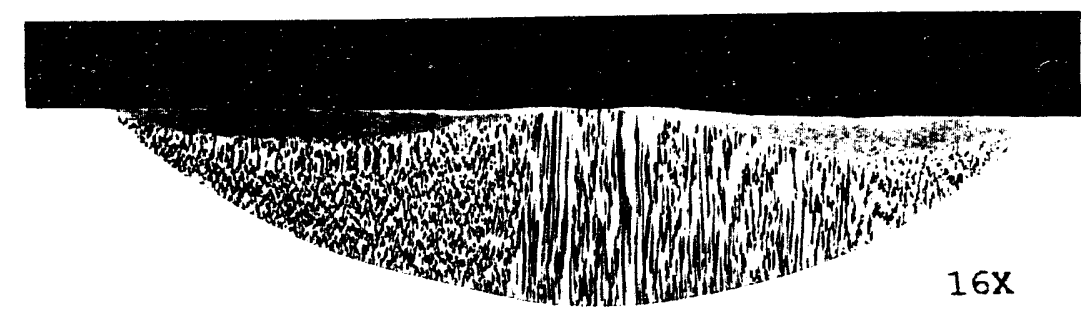

(c)

Transverse View GTA Stationary Weld [110] Direction

Figure 26

Three-dimensional Reconstruction of (001) Plane [110] Direction

Stationary GTA Weld With Accompanying Macrographs 
of the weld pool in the [001] direction. Figure 26a shows the three-dimensional reconstruction from the macrographs. It is also noted that the angles for the dendritic growth are illustrated on this figure.

Figure 27 represents the three-dimensional reconstruction with higher magnification areas " $A$ " and "B" indicated as shown. Area "A" and "B" in Figure 27 is represented microstructurally in Figures 28 and 29 respectively. Figure 28 illustrates the symmetry of the weld pool shape and shows the [100] dendrites and the [001] dendrite growths. The same velocity comparison of angles and varied growth rates can be made here as in the previous direction in order to explain this microstructural formation. This can be compared to the photomascograph of the [100] transverse view shown in Figure 19. It can be seen that each weld pool and microstructure are symmetrical. This means that the weld microstructure and the weld pool are repeatable from weld-to-weld. Figure 29 denotes the [001] dendritic growth in the center section of the weld illustrating the uniformity of the dendrite spacing and also, shows that only one dendritic growth was present in this area, which means that the thermal gradients were aligned to favor only one of the preferred growth directions for this region. 


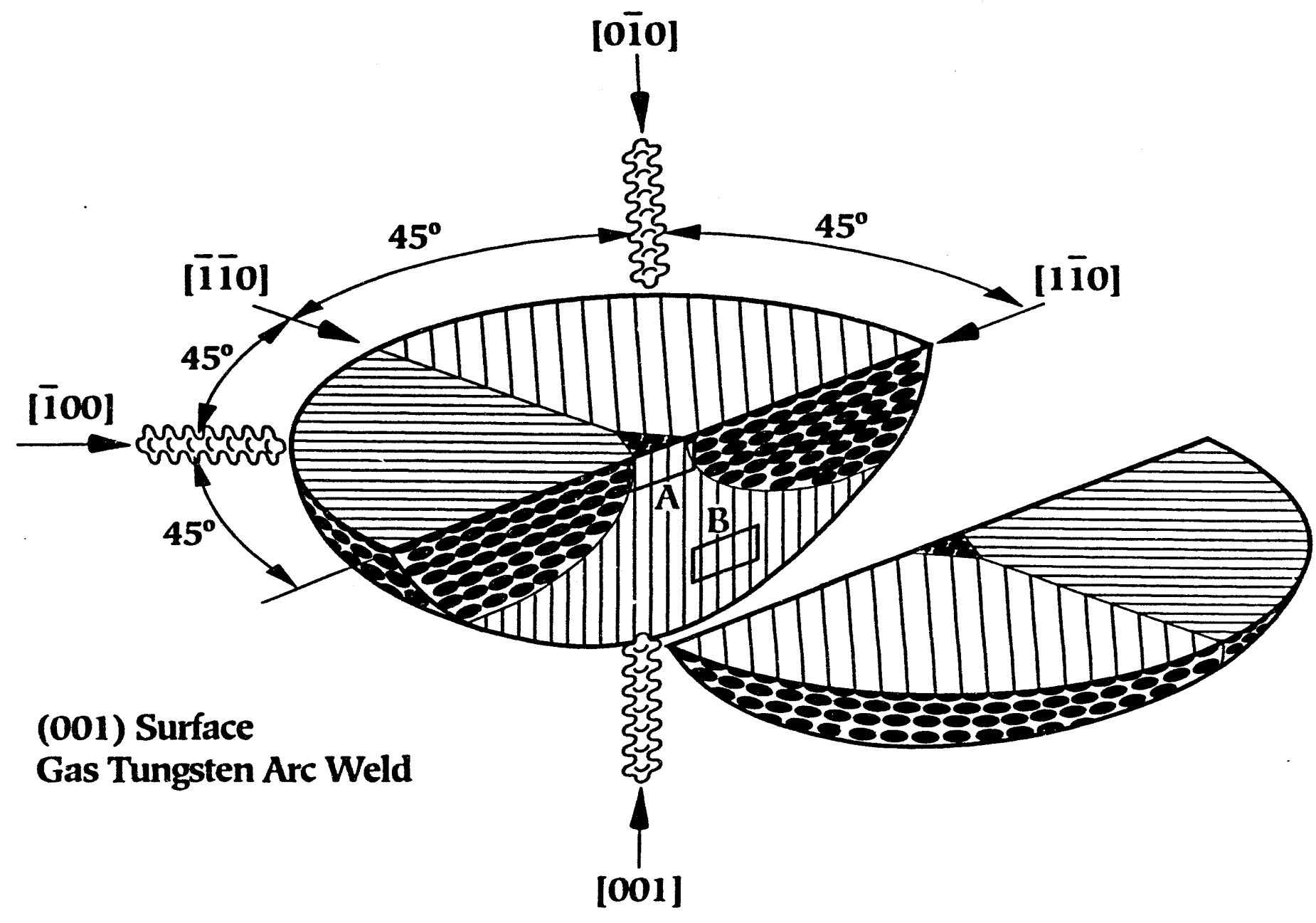

Figure 27

Three-dimensional Reconstruction Identifying 


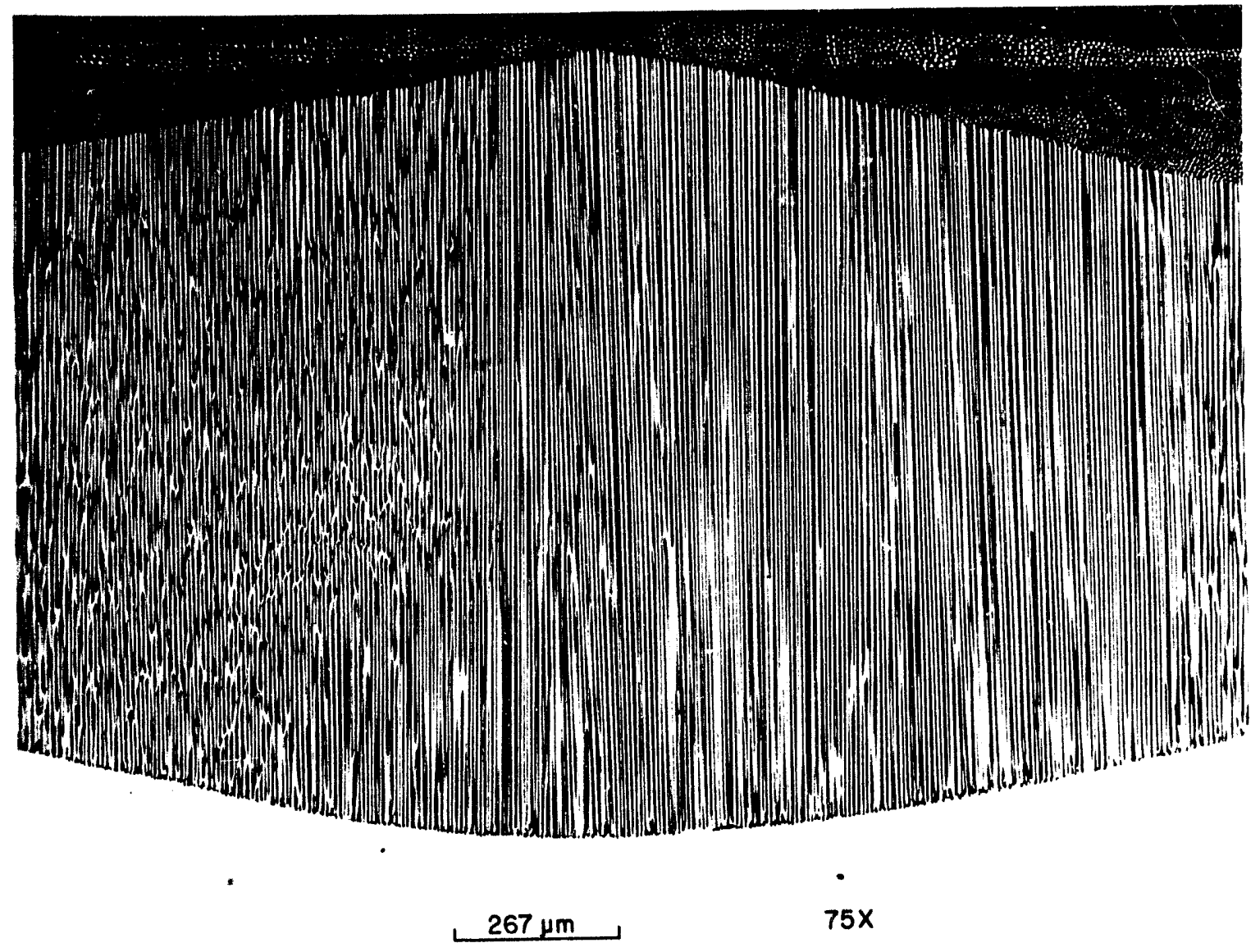

Figure 28

Center section of [110] Transverse View

(Area "A" of Figure 27) 
7

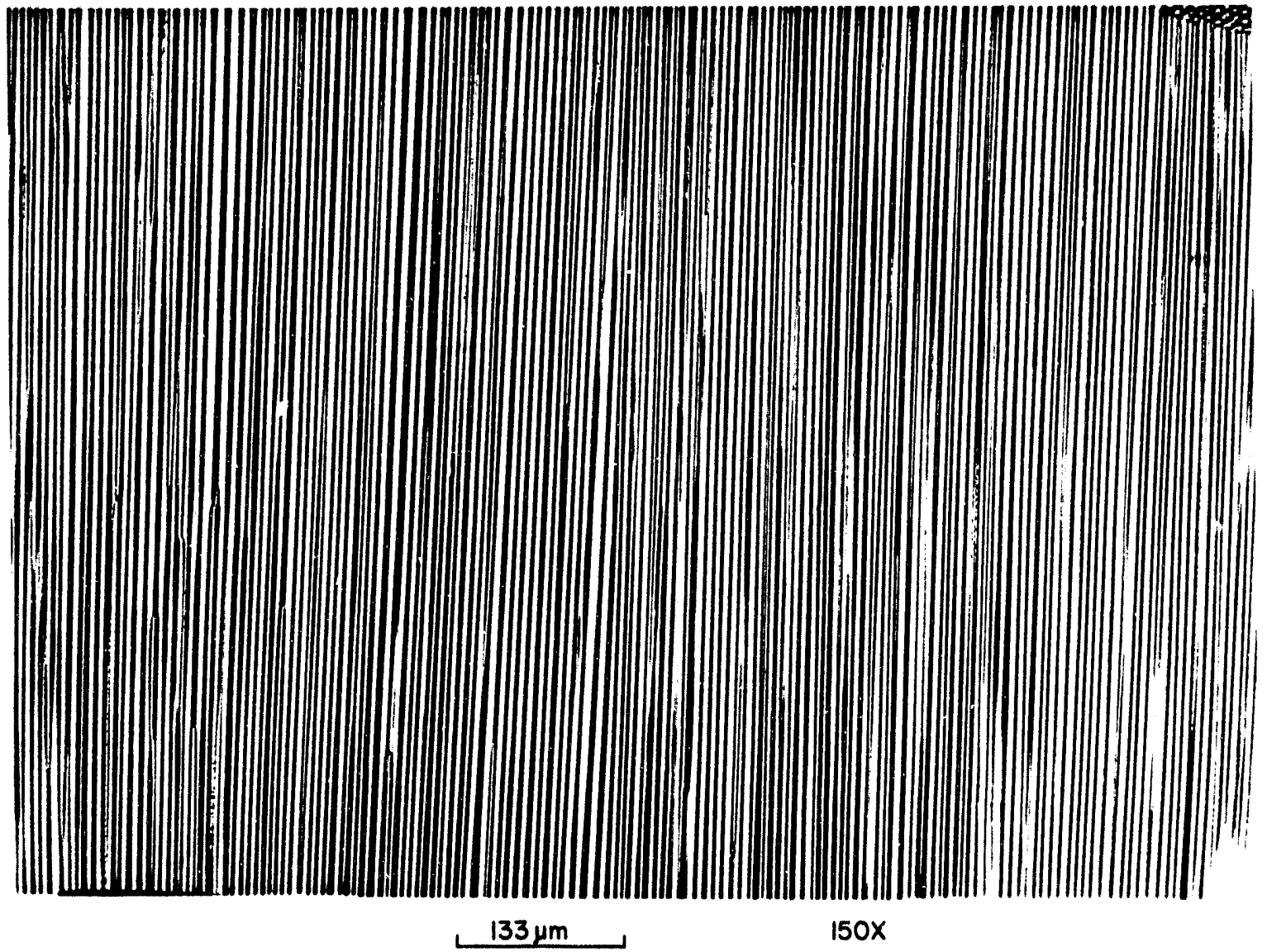

Figure 29

Center section of [110] Transverse View

(Area "B" of Figure 27) 
Summary of (001) Orientation

A combination of these three views, i.e., the [100] direction, surface depth, and [110] direction, confirms that solidification takes place on the top surface from the edge of the weld inward along the [100] and [010] directions in area "a" and "b" (F1gure 15a). This solidification pattern proceeded downward into the weld. Simultaneously, solidification occurred along the sides and bottom of the melt pool in the [001] direction in area "c". A section of this growth proceeded to the surface of the weld. The rest of the dendrites were terminated when encountering either the [100] or the [010] dendrites.

\section{(011) Crystallographic Orientation}

The stereographic projection for the (011) plane appears in Figure 30 [33]. Rectangular areas denote the preferred dendritic growth directions for this crystallographic orientation. Each of the $\langle 100\rangle$ directions are $180^{\circ}$ apart. The [100] directions are 900 from the interior of the crystal are the [001] and [010] directions. The dendritic growth along these directions are at an angle of $45^{\circ}$ upward from the crystal surface [34]. These directions, [100], [001], and [010] are the preferred growth directions for the (011)-surface of the FCC single crystal and yielded a two-fold symetric structure. 


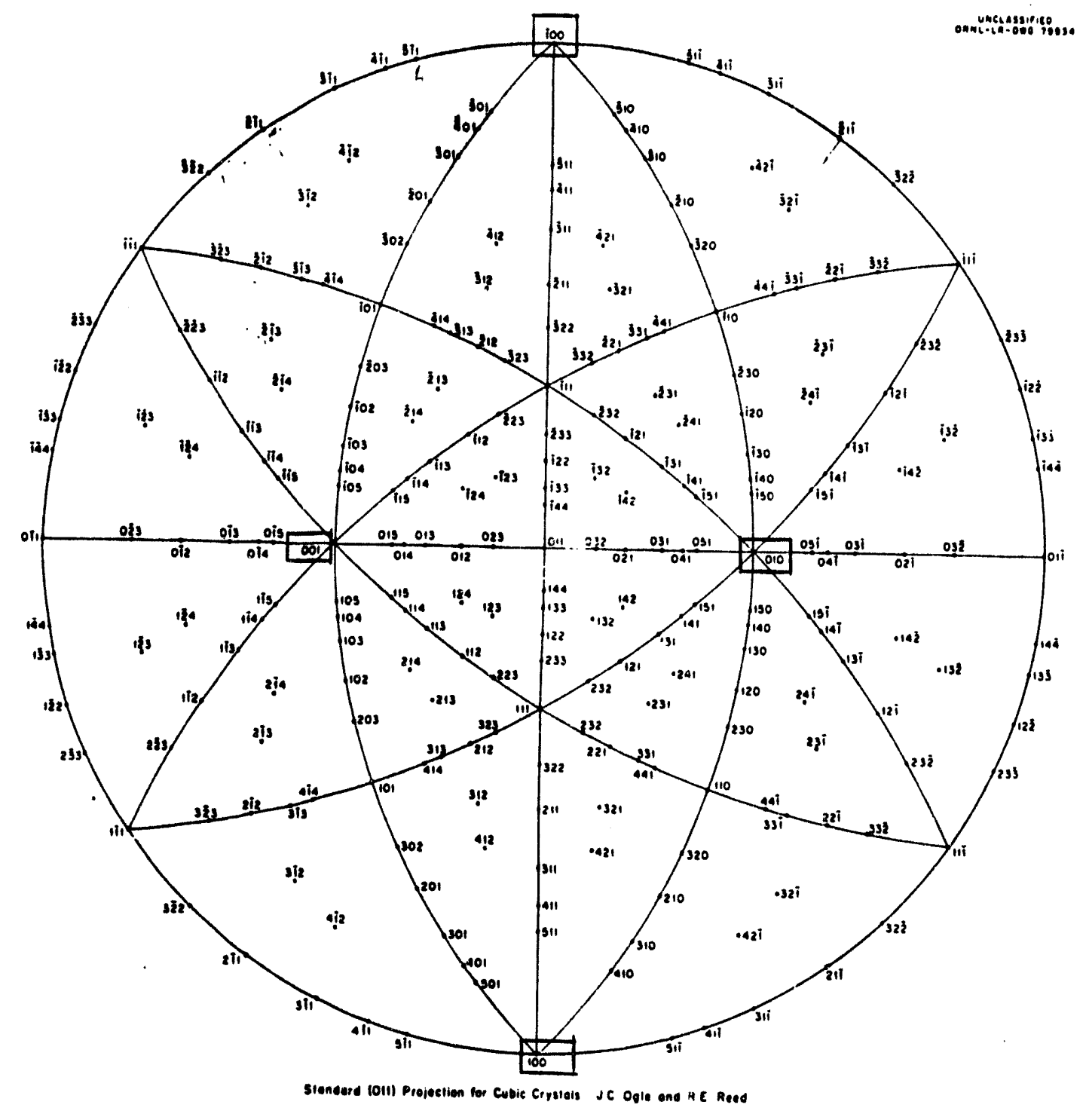

Figure 30

Stereographic Projection of the (011) Plane 
GTA partial-penetration stationary welds on this surface provided three cross-sectional views along the [100], [110], and [111] directions combined with an "in-depth" surface view as described in the previous section. These four views were combined to form a three-dimensional reconstruction of the resultant microstructure for a GTA stationary spot weld on the (011) surface.

Sectioning Along the $[100]$ Direction: Figure 31 shows the welding configuration and sectioning for the [100] primarydirection transverse view. As illustrated, the heat source was used to obtain a partial penetration melt pool on the (011) surface. The three-dimensional reconstruction with the accompanying photomacrographs which aided in creating the reconstruction is illustrated in Figure 32. A top-surface view revealed the two-fold symmetry involved in this crystallographic orientation (Figure 32b). This photomacrograph shows the [100] dendrites growing across the weld surface as well as the [001] and [010] dendrites growing upward forming elliptical crosssections in the surface view. The [100] transverse view (Figure 32c) revealed the [100] dendrites growing across the pool and the [001] dendrites forming circular sections on the cross-sectional view. From these two views, it was determined that the interior dendrites were not growing perpendicular to the surface as in the (001) plane, becauge there was only the cross-section of the 


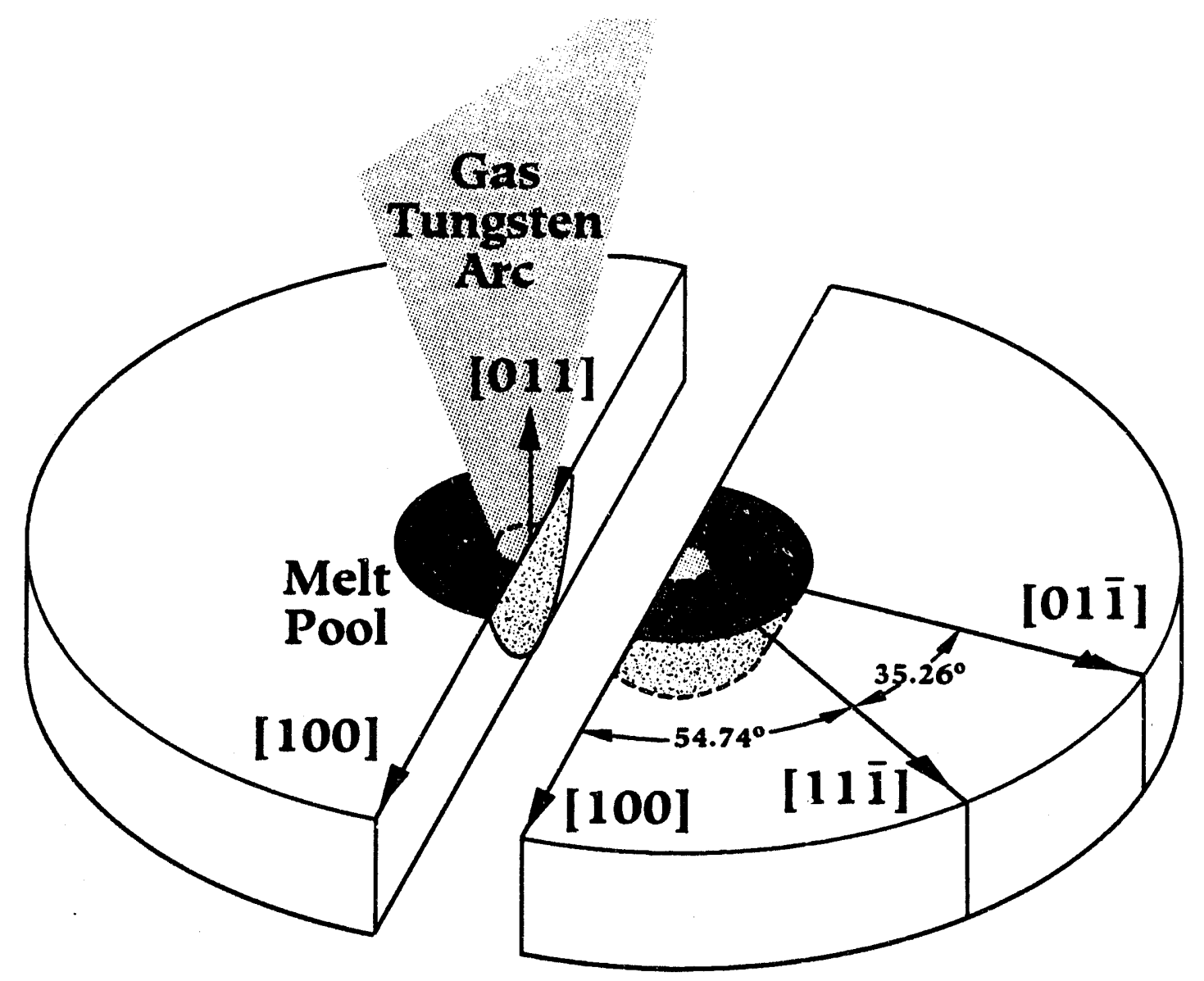

Stainless Steel Single Crystal (011) Surface

Figure 31

Schematic for (011) Plane [100] Direction

Sectloning and Welding Configuration 


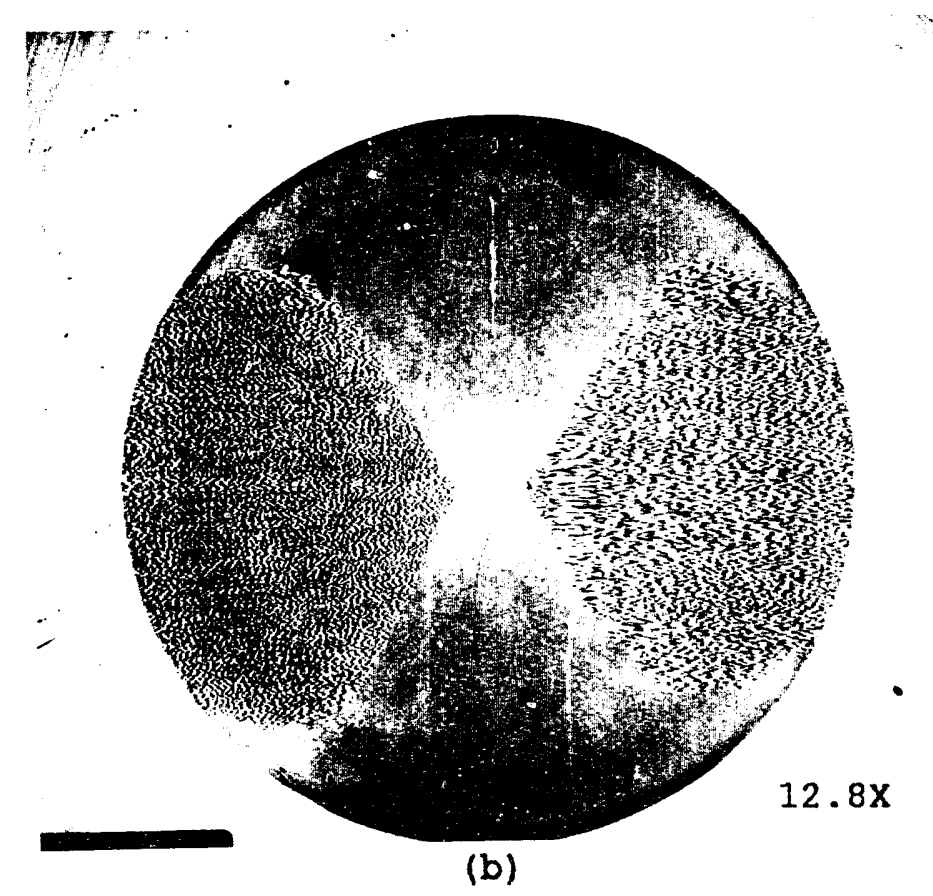

(011) Surface View GTA Stationary Weld Top Surface

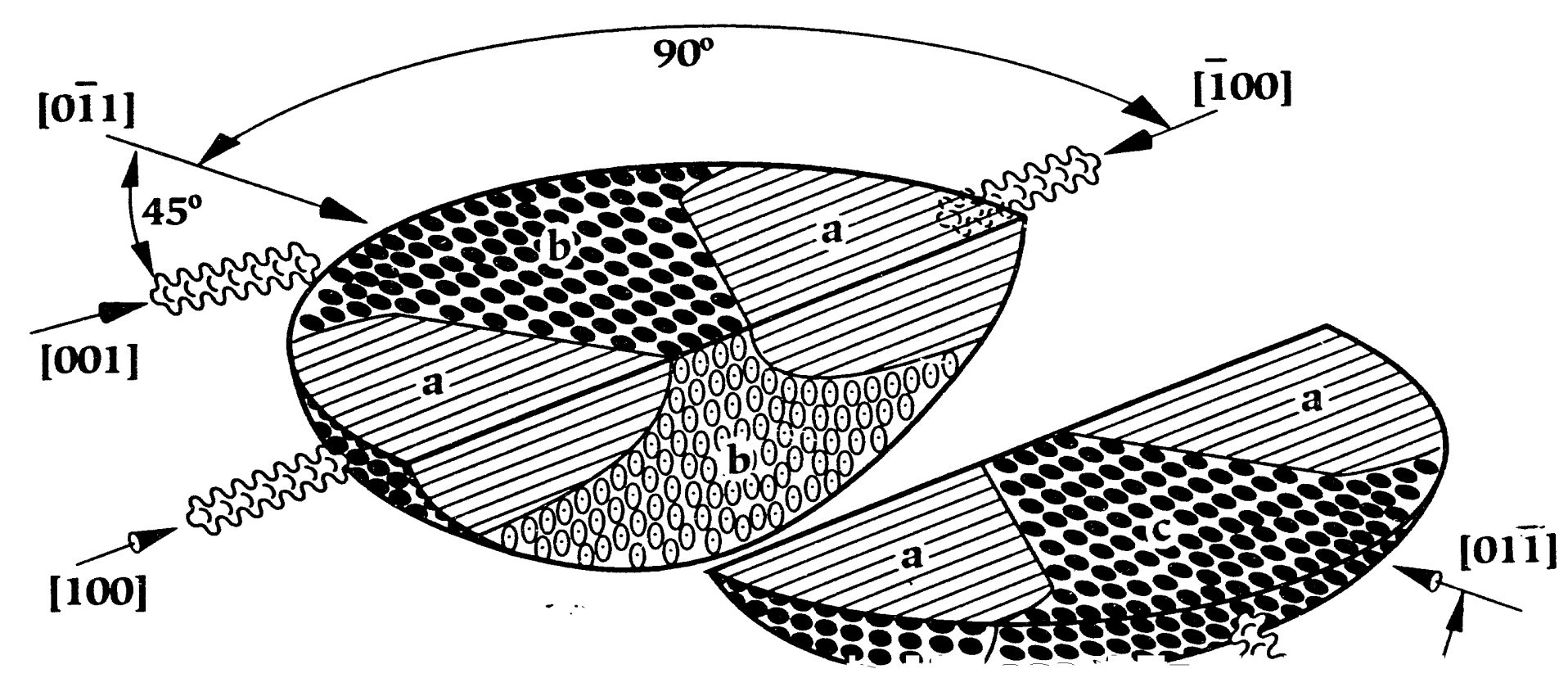




\section{(01 1) Surface}

Gas Tungsten Arc Weld

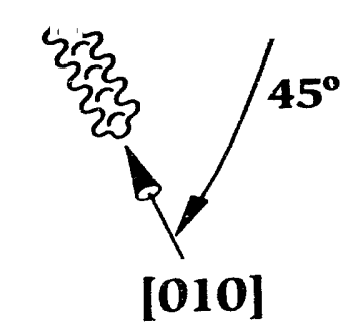

(a)

Three-dimensional Reconstruction of the Resultant Microstructure

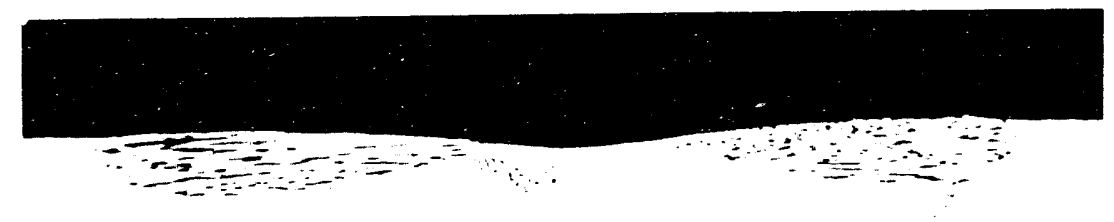

$16 \mathrm{X}$

(c)

[100] Direction Transverse View (110) Plane

GTA Stationary Weld

Figure 32

Three-dimensional Reconstruction of (011) Plane [100] Direction

stationary GTA Weld with Accompanying Photomacrographs 
dendrites present in each view. Instead, they were growing at an angle to the surface. Using the stereographic projection, it was determined that dendrite growth was along the two interior $\langle 100\rangle$ directions at a $45^{\circ}$ angle. These two views are combined to construct the three-dimensional representation of the resulting microstructure (Figure 32a) for this transverse direction view showing the angles for the dendritic growth. Dendrites grew in areas " $a$ " dendrites grew along the [100] directions. In areas " $b$ " dendrites grew along the [001] direction and in area " $c$ " dendrites grew along the [010] direction.

Areas " $A$ " and "B" denote higher-magnification photomicrographs for the solidification features or dendrite appearance which are presented in Figure 33. Area "A" of Figure 33 is microstructurally represented in Figure 34 . Figure 34 illustrates the intersection of the [100] and [001] dendrites in the weld. Competition and dendrite velocities are evident in the pattern of dendrite solidification. The [100] dendrites grew across the weld until encountering the interior $<100\rangle$ dendrites. This illustrates the role of dendrite velocity variations in the formation of the microstructure as discussed previously. The photomicrograph of area "B" on Figure 33 is microstructurally represented in Figure 35 and shows a solidification feature of the weld. Where a section of the [010] dendrites grew into the sample. Consequently, a section of [100] dendrites grew parallel 


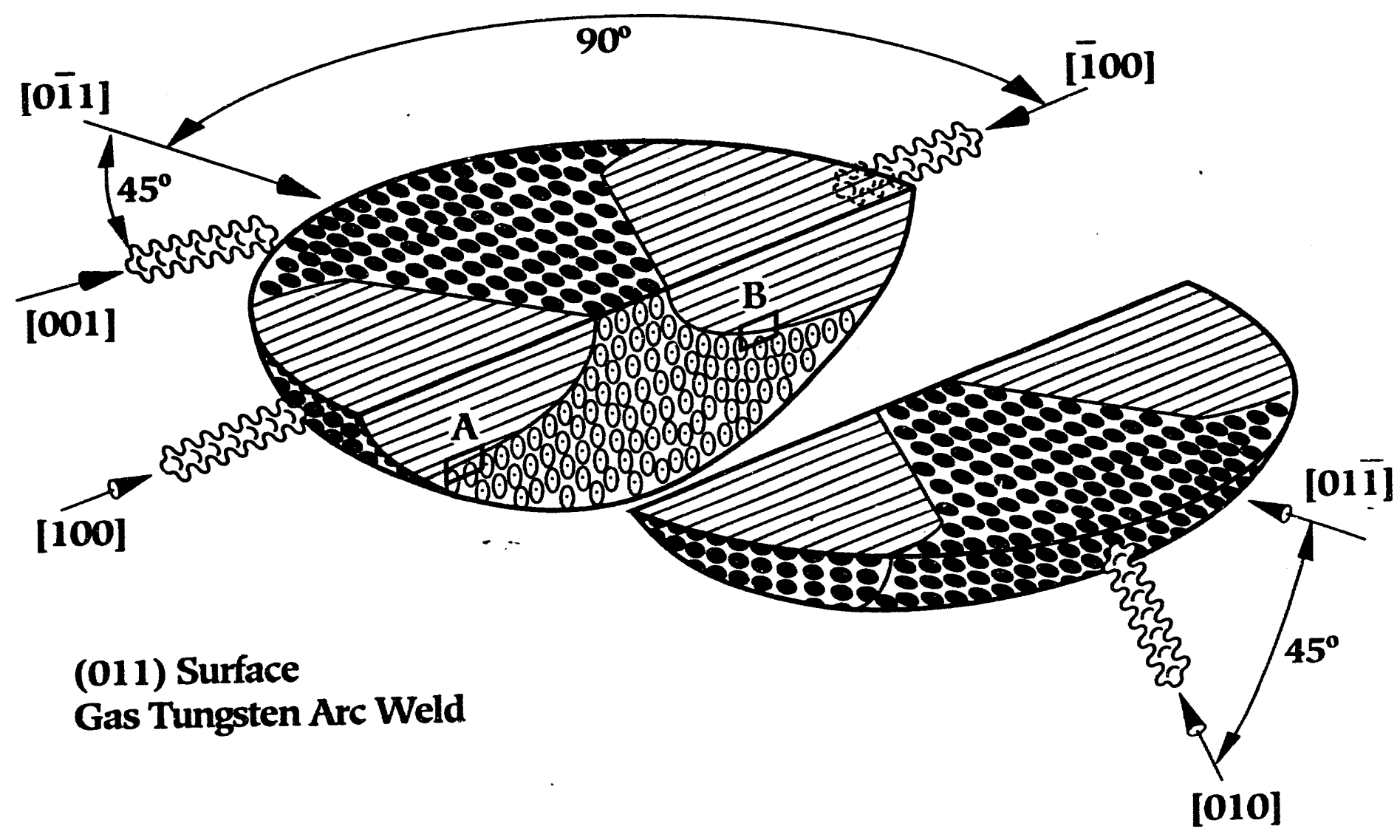

Figure 33

Three-dimensional Reconstruction Identifying

High Magnification Areas 


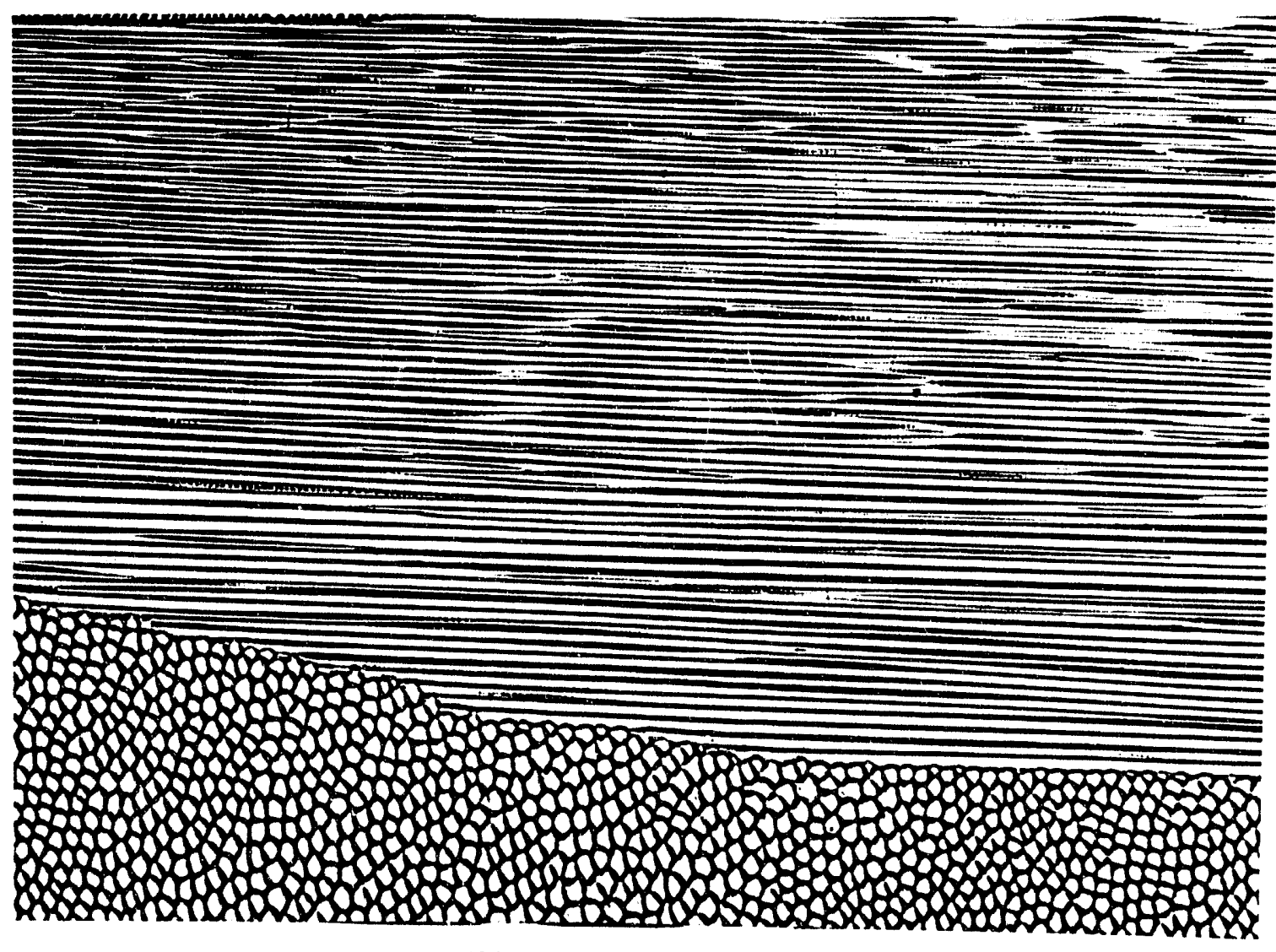

$100 \mu \mathrm{m}$

$200 x$

Figure 34

(011) Stationary GTA Weld

[100] Transverse View for Solidification Features

(Area "A" of Figure 33) 


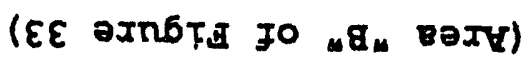

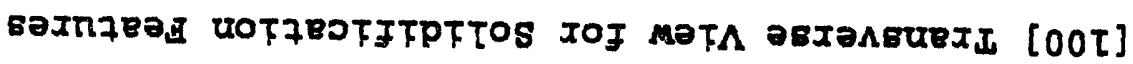

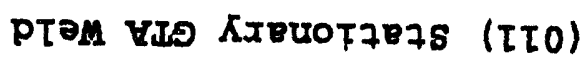

SE จxก6下]

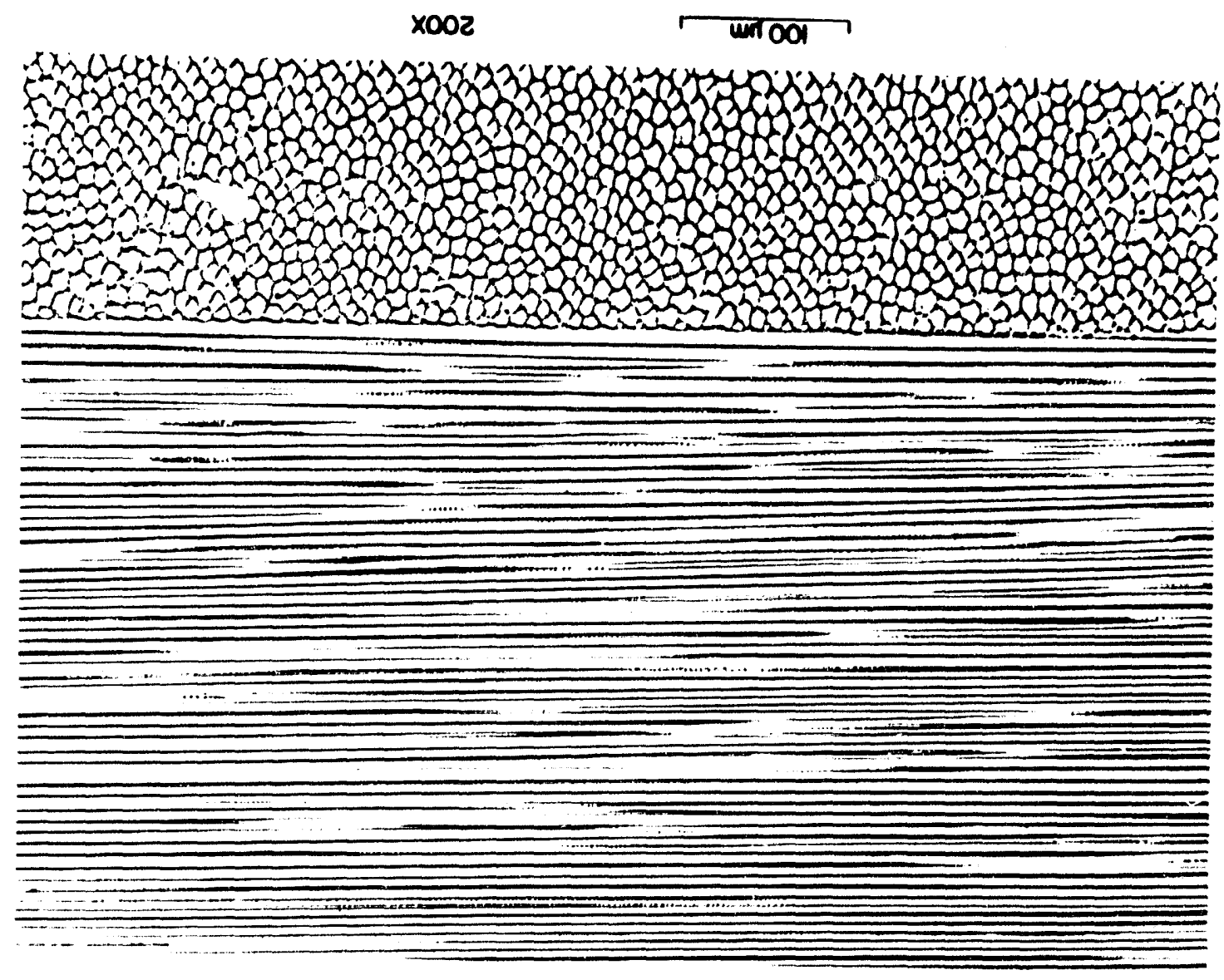


to the surface. From this solidification pattern, it was concluded that the [010] dendrites were present before the [100] dendrites solidified because the new dendrites grew until encountering dendrites which terminated their growth.

Top surface: Top surface views of the (011) plane for the GTA stationary welding configuration are schematically illustrated in Figure 36. Noted in this figure are the primary directions which aided in the microstructural analysis. Depicted in Figure 37 is the three-dimensional reconstruction of the resultant microstructure for a GTA stationary weld on a (011) surface, accompanied by a series of surface views at various depths into the weld. The top-surface view revealed the two-fold symmetry involved in this crystallographic orientation (Figure 37b). The dendrites parallel to the surface grew along the [100] directions and the dendrites at an angle to the surface grew along the [001] and [010] directions. Further into the weld it is seen that the concentration of the [001] and [010] dendrites increases by decreasing the $[100]$ dendrite concentration (Figure 37c). This process of increasing concentration of the [001] and [010] dendrites continued until the surface was completely covered (Figure 37d). Even though the entire surface was covered with dendrites growing along the interior set of <100> directions, the two-fold symmetry was still evident (Figure 37d). 


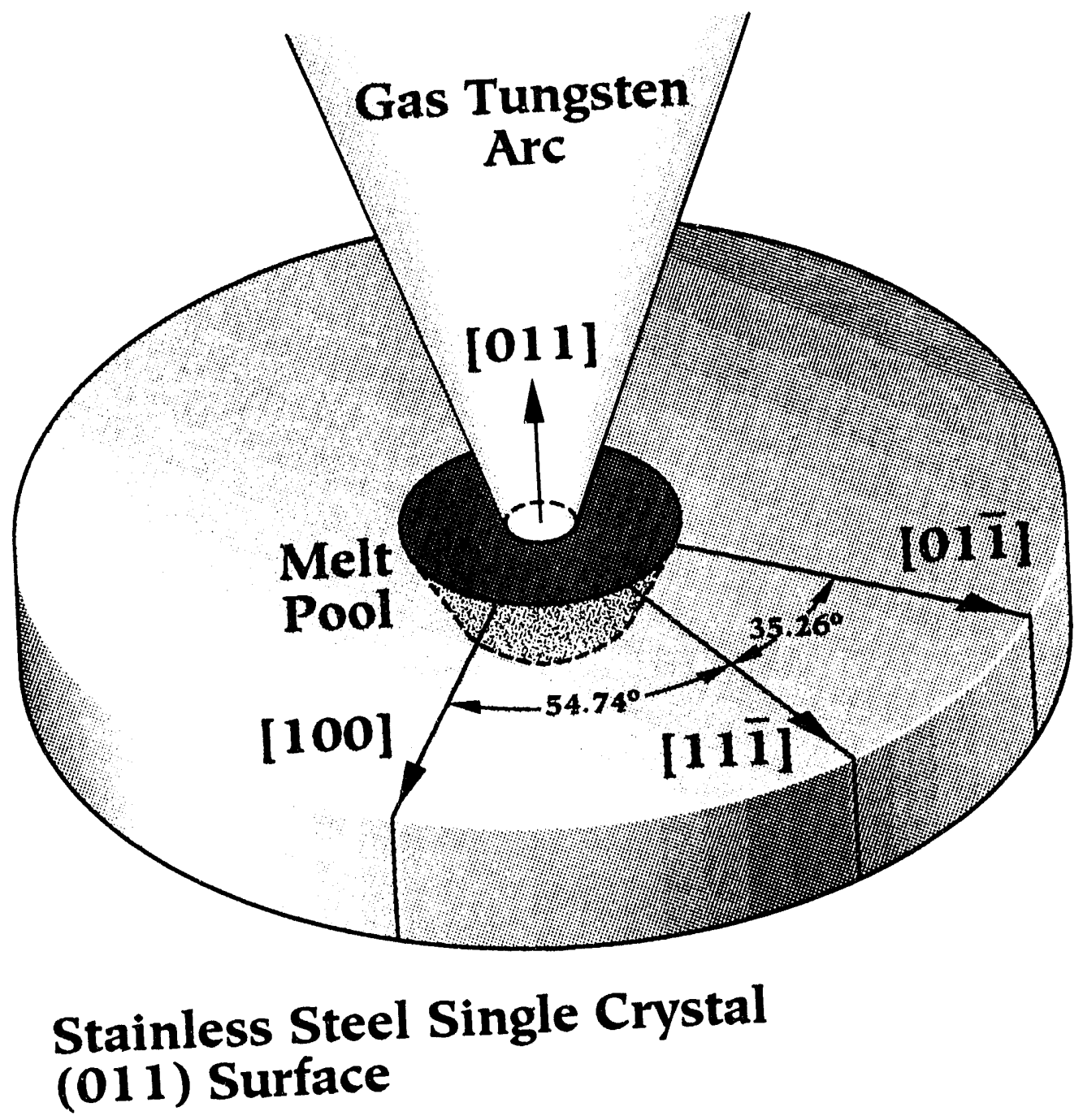

Figure 36

Schematic for the (011) Plane Welding Configuration 


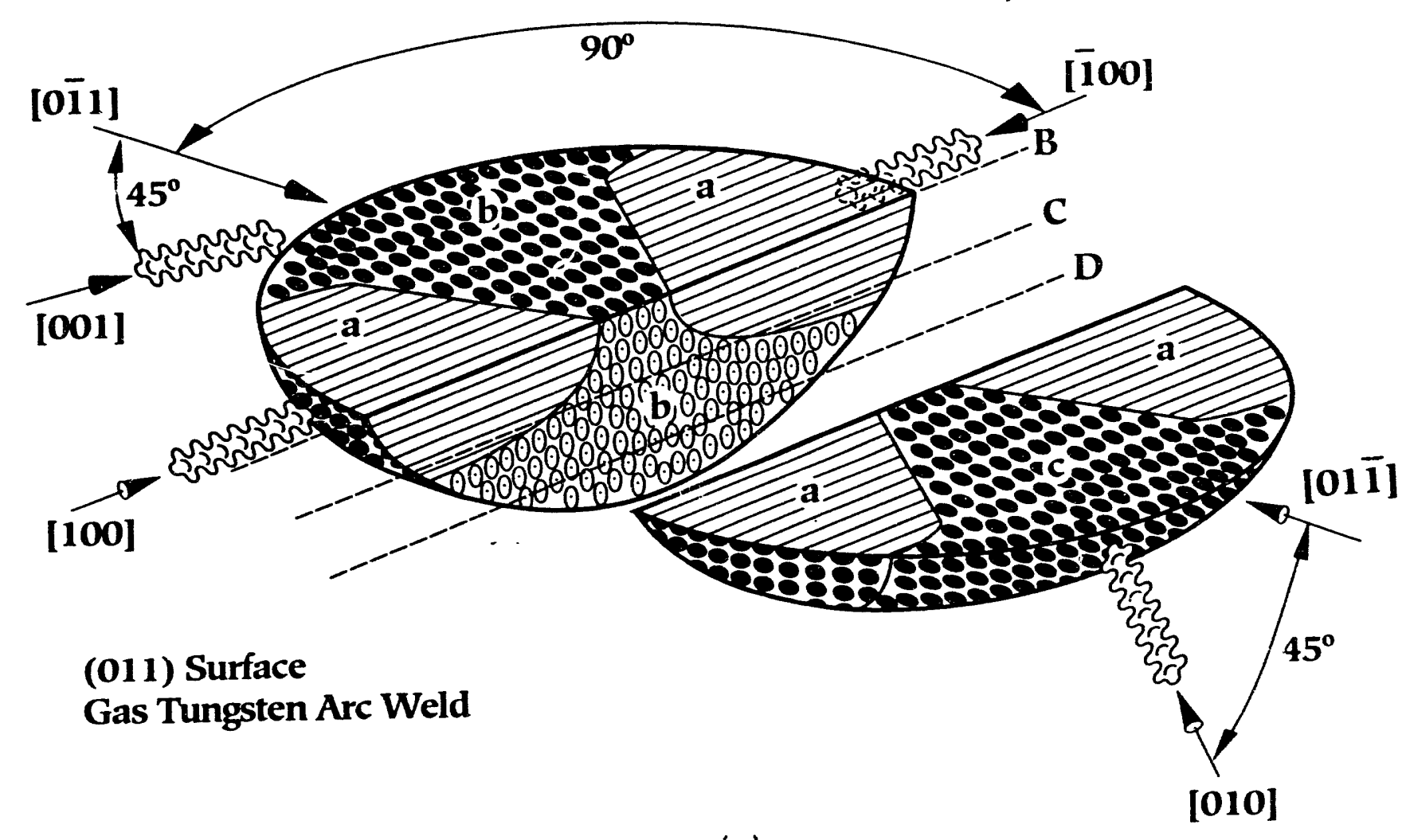

(a)

Three-dimensional Reconstruction of the Resultant Microstructure

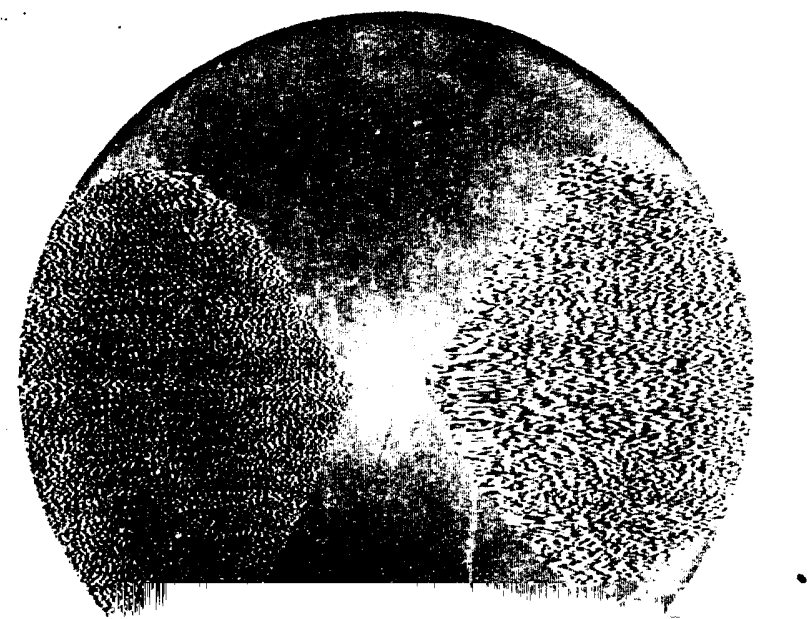


(011) Surface V1ew GTA Stationary Weld Top Surface

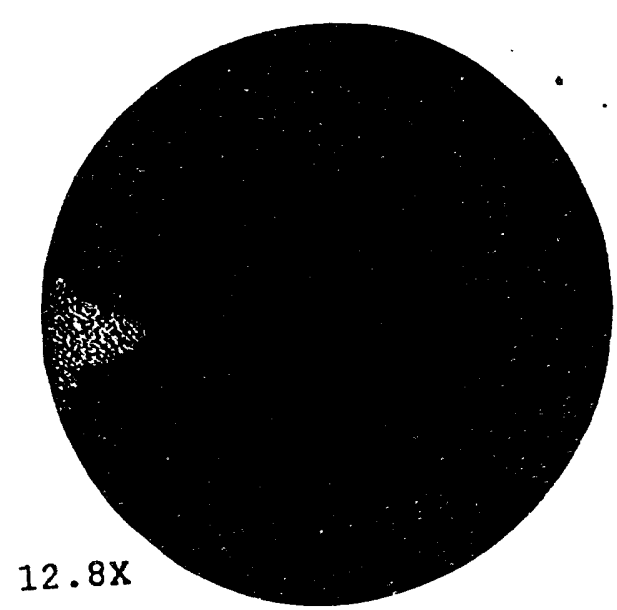

(c)

(011) GTA Stationary Weld

Increased Depth into Top Surface

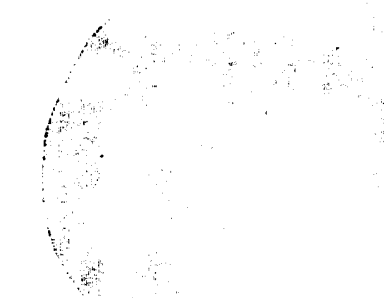

$12.8 x$

(d)

(011) GTA Stationary Weld Complete Coverage of Dendrites

\section{Figure 37}

Three-dimensional Reconstruction of (011) Plane Surface stationary GTA Weld with Accompanying Photomacrographs 
The three-dimensional reconstruction (F1gure 37a) was determined from these surface views, the cross-sectional views, and the stereographic projection. This figure notes the directions and angle for the dendritic growth. It should be noted on this figure that the lines through the weld mark the approximate area in which the suxface views were taken. It shows that in areas "a" the dendrites grew along the [100] direction. In area "b", there was [001] dendritic growth and in area " $c$ ", dendrites grew in the $[010]$ direction. An important note was that growth in areas " $b$ " and " $c$ " did not cross the weld surface; 1.e., they terminated on each other at the centerline of the melt pool. Figure 38 illustrates the areas $" A n, " B n$, and " $C "$ in which higher-magnification micrographs were used to illustrate the symmetry of this weld. Area "A" of Figure 38 is illustrated in Figure 39 and shows the center section of the weld with the [100] dendrite and the interior preferred dendrites, [001] and the [010] are visible. This microstructure illustrates the symmetry of the weld microstructure as well as the comparatively symmetric growth velocity of each half. Areas "B" and "C" in Figure 38 are microstructurally represented in Figure 40 and 41 respectively. They represent the left and right halves, respectively, indicating the growth across the surface in the [100] direction meeting either the [001] or the [010] dendrites. These figures 


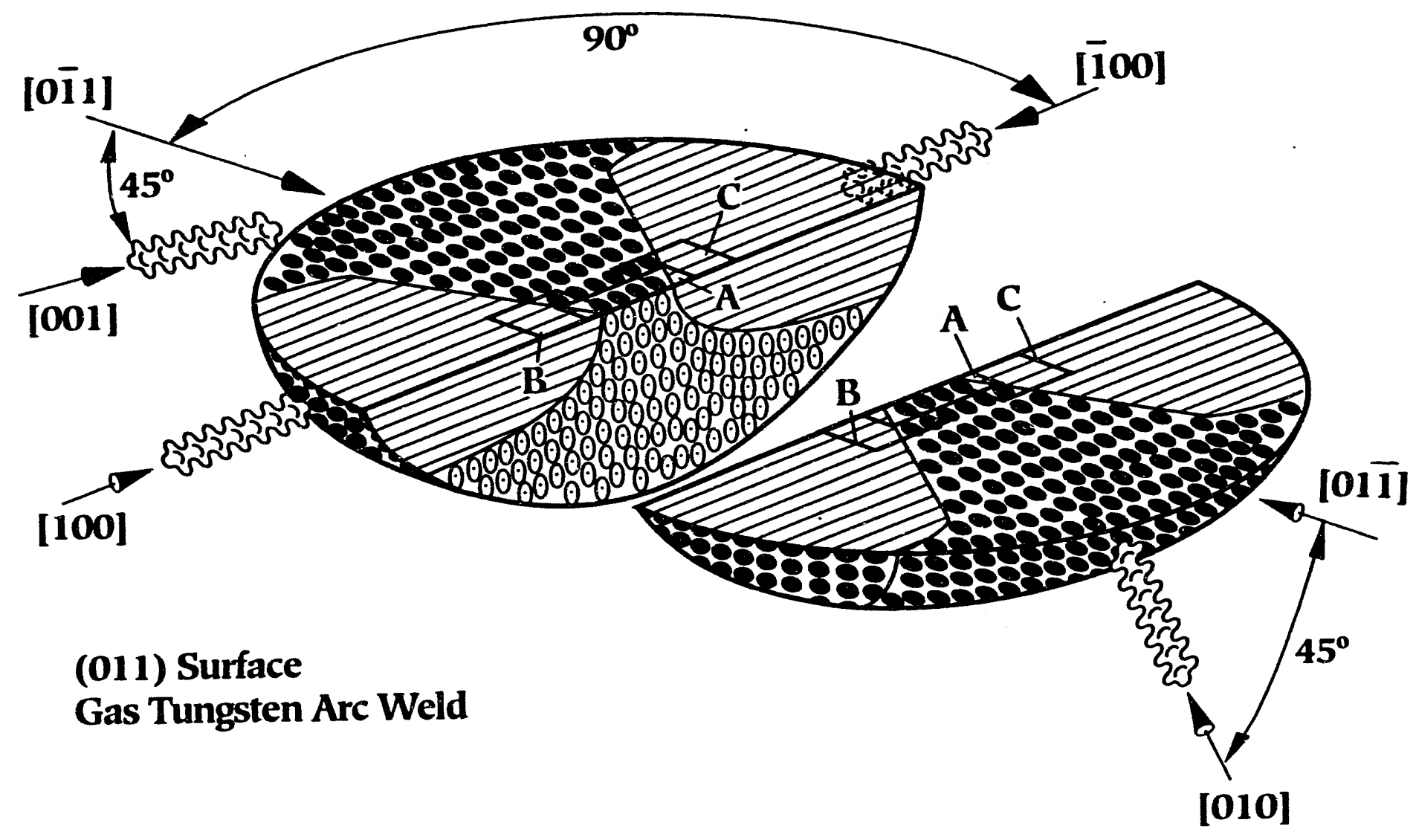

Figure 38

Three-dimensional Reconstruction Identifying

High Magnification Areas 


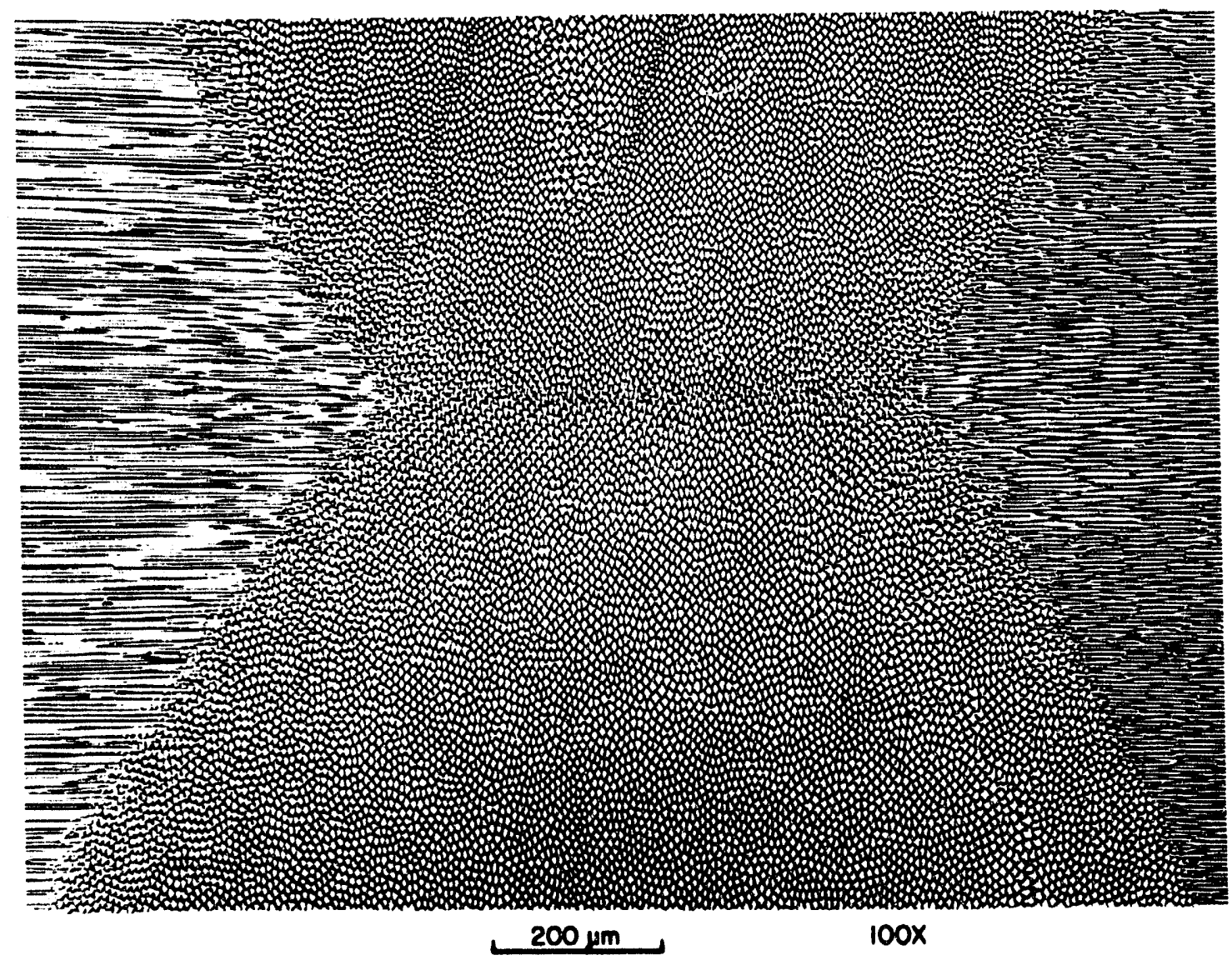

Figure 39

Center section of (011) surface Weld

(Area "A" of F1gure 38) 


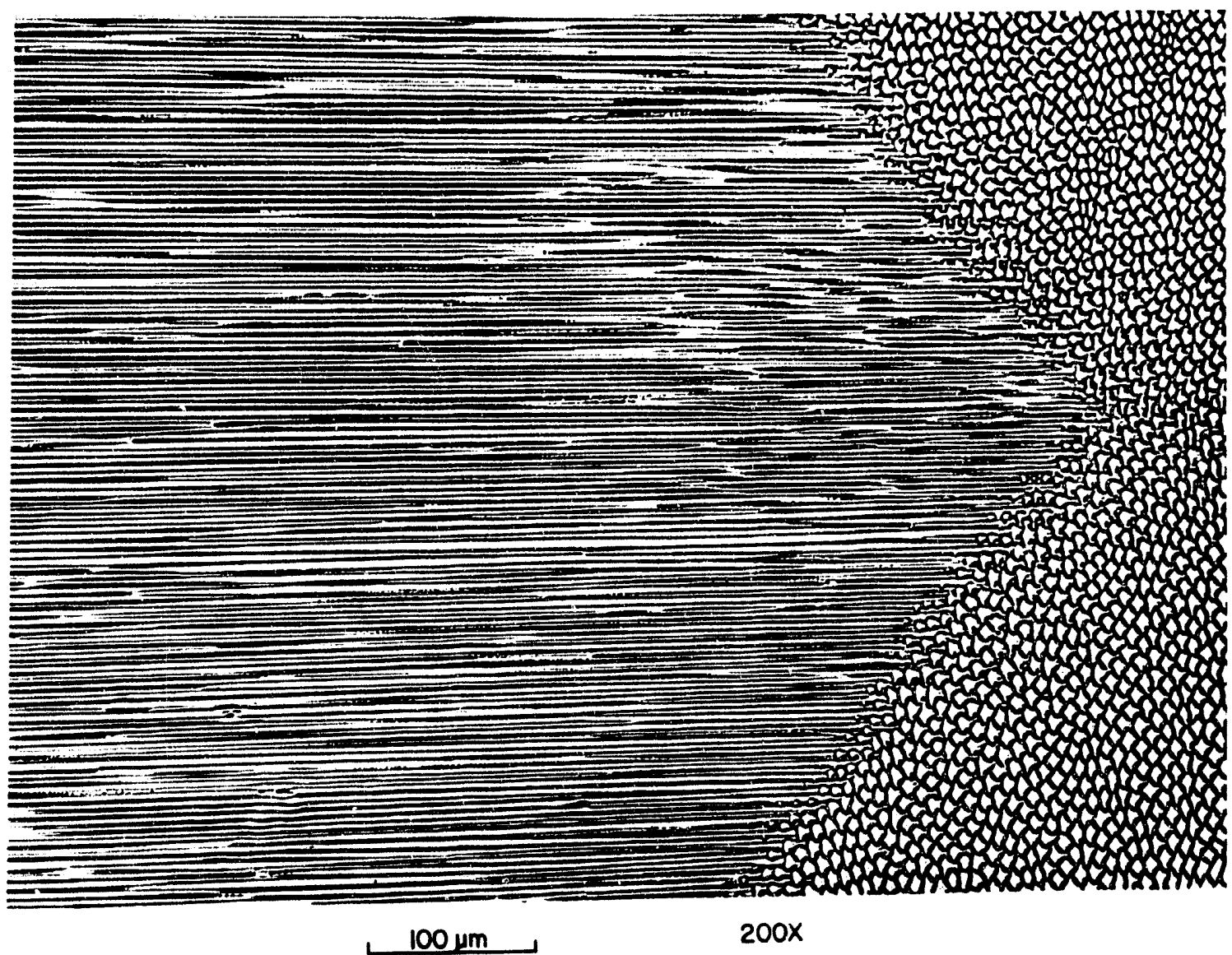

Eigure 40

Left side of Center section (011) Surface Weld

(Area "B" of Figure 38) 


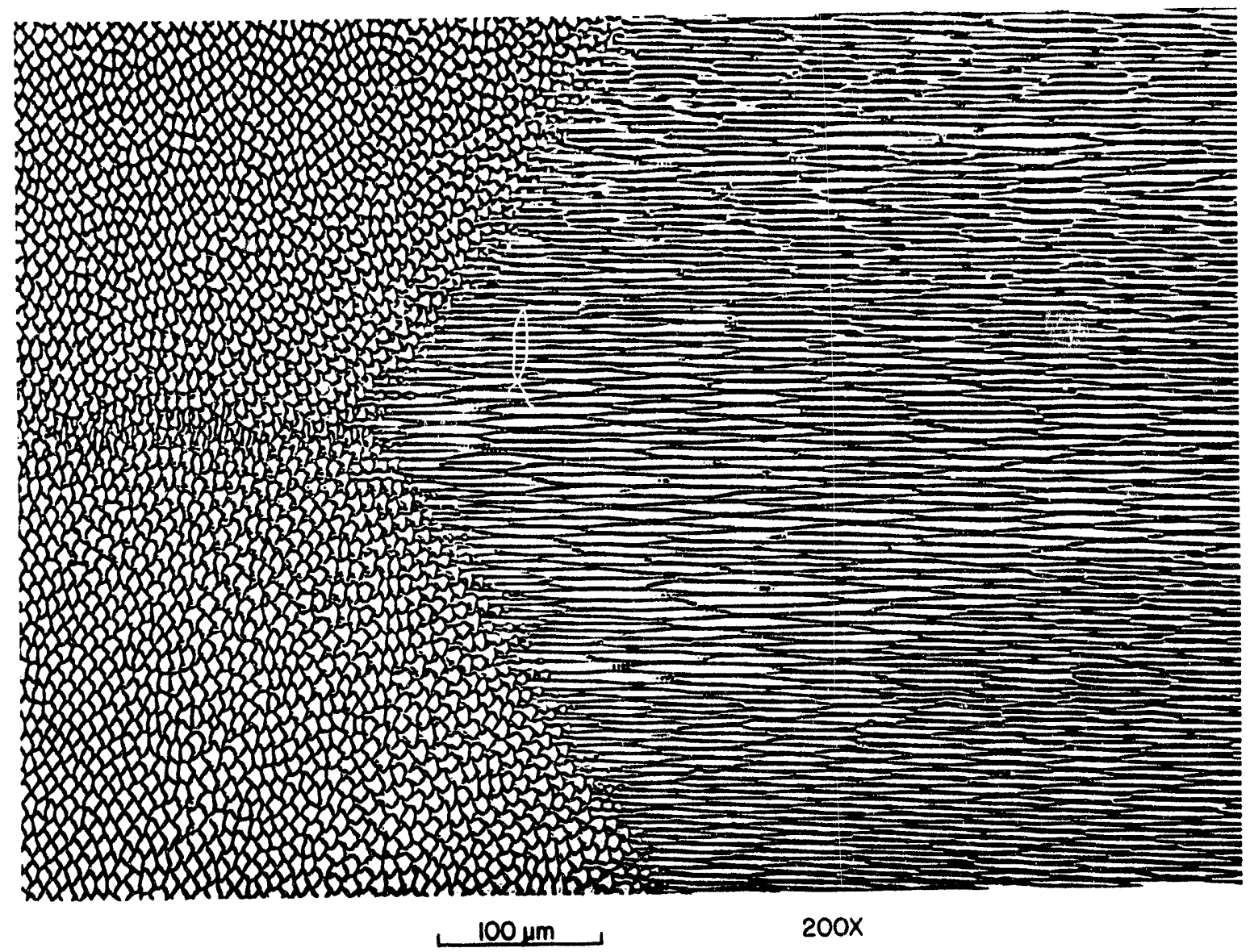

Figure 41

Right Side of Center section (011) Surface Weld

(Area "C" of Figure 38) 
show the symmetry involved in these welds and the dendrite growth pattern.

Sectioning Along the [110] Direction: The GTA stationary welding configuration and sectioning of the weld for the crosssection of the [110] direction is represented schematically in Figure 42. A three-dimensional reconstruction of the resultant microstructure for this direction is depicted, with the accompanying photomacrographs, in Figure 43. The surface view shows the [100] dendrites growing across the weld and the [001] and [010] dendrites in cross-section. It was observed in the [110] transverse view that dendritic growth occurred only along the [001] and [010] directions and met at the weld center (Figure 43c). The [110] transverse view illustrates the previous statements that the dendrites grew in the [001] and [010] directions and terminated at the center of the weld pool. This figure clearly shows the solidification patterns of the [001] and [010] dendrites as growing from the edges of the weld toward the weld surface. The three-dimensional reconstruction, from the microstructures, is shown in Figure 43a. Also, noted in this figure are the angles at which dendritic growth occurred in each direction.

Figure 44 indicates area "A" in which a higher-magnification micrograph illustrated the dendrite-growth phenomena. Area "A" of Figure 44 is microstructurally represented in Figure 45 . This 


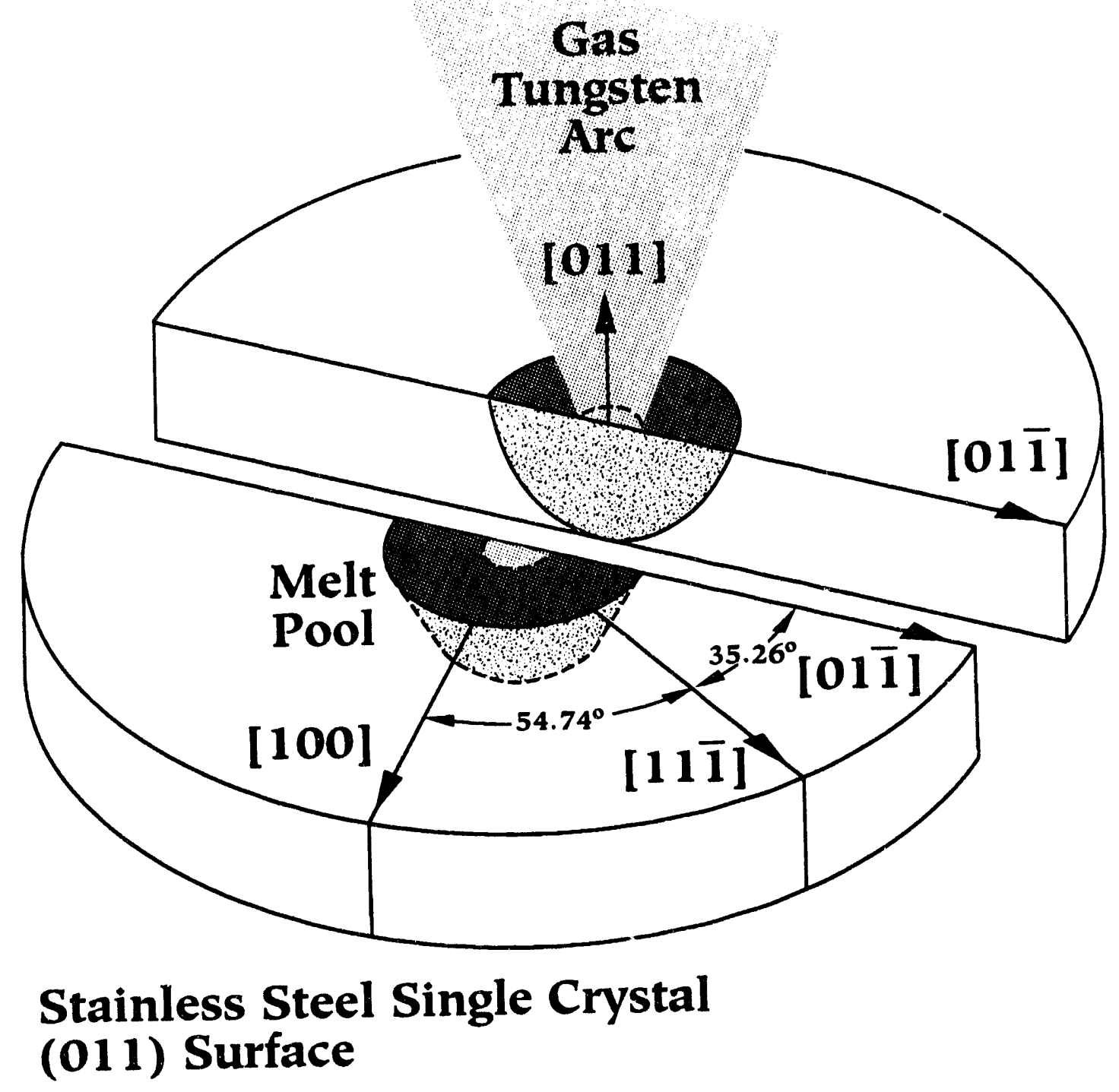

Figure 42

Schematic for (011) Surface [110] Direction

Sectioning and Welding Configuration 


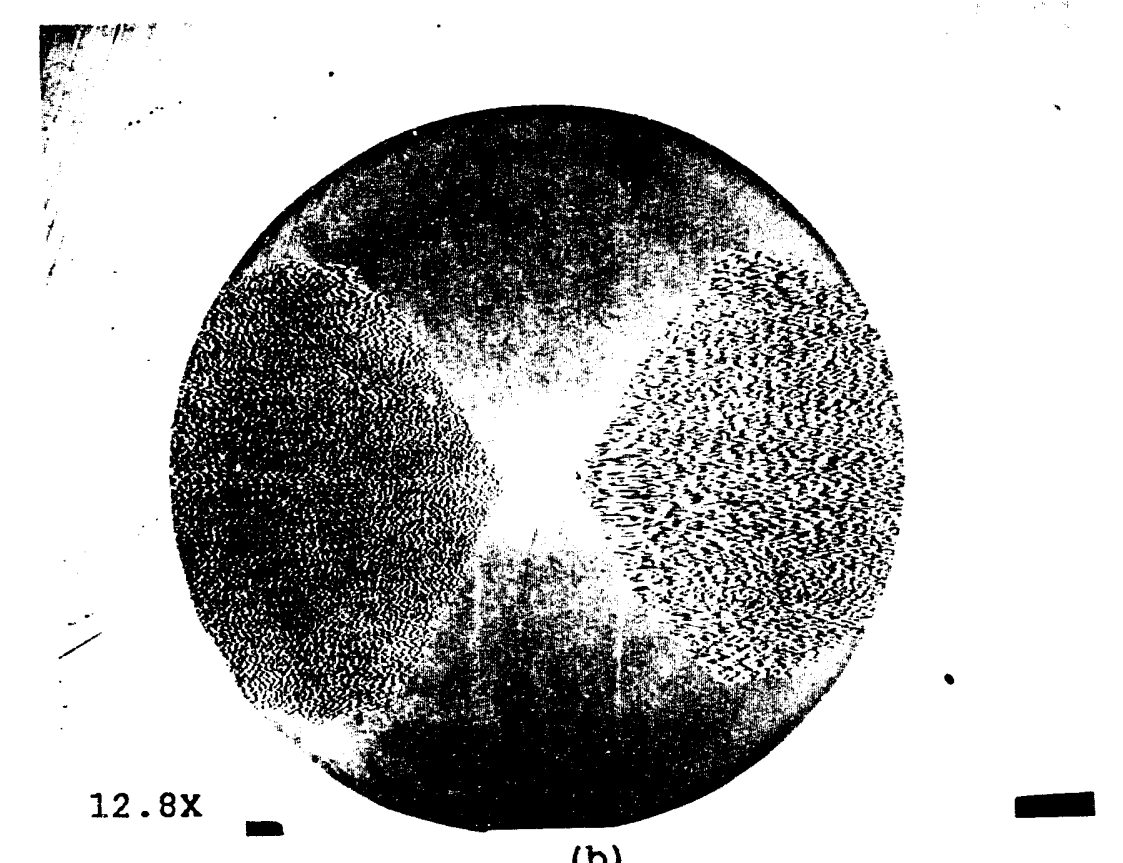

(011) Surface View stationary GTA Weld Top Surface

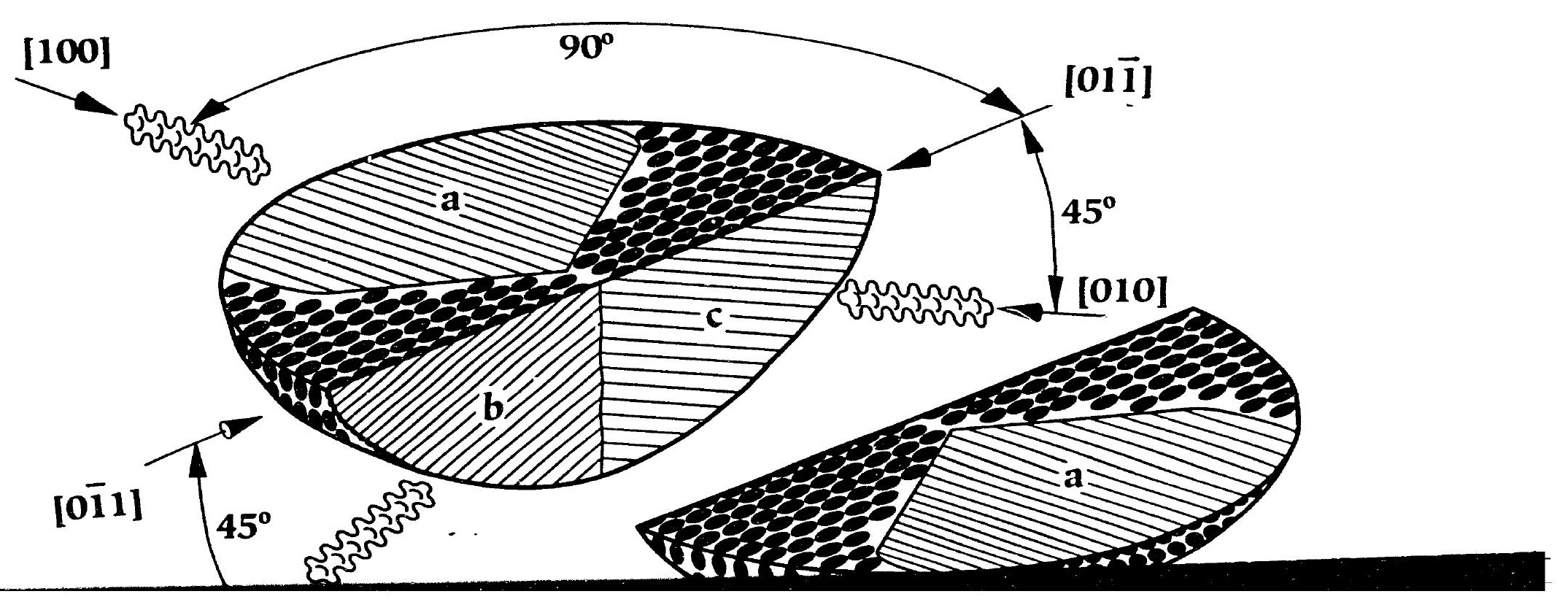




\section{[001]}

(01 1) Surface

Gas Tungsten Arc Weld

Figure (a)

Three-dimensional Reconstruction of the Resultant Microstructure

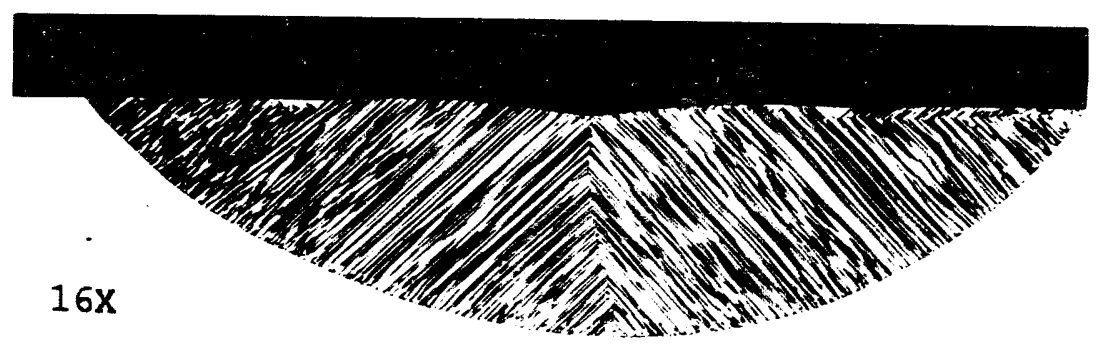

(c)

[110] Direction Transverse View (110) Plane

GTA stationary Weld

\section{Figure 43}

Three-dimensional Reconstruction of (011) Plane [110] Direction Stationary GTA Weld with Accompanying Photomacrographs 


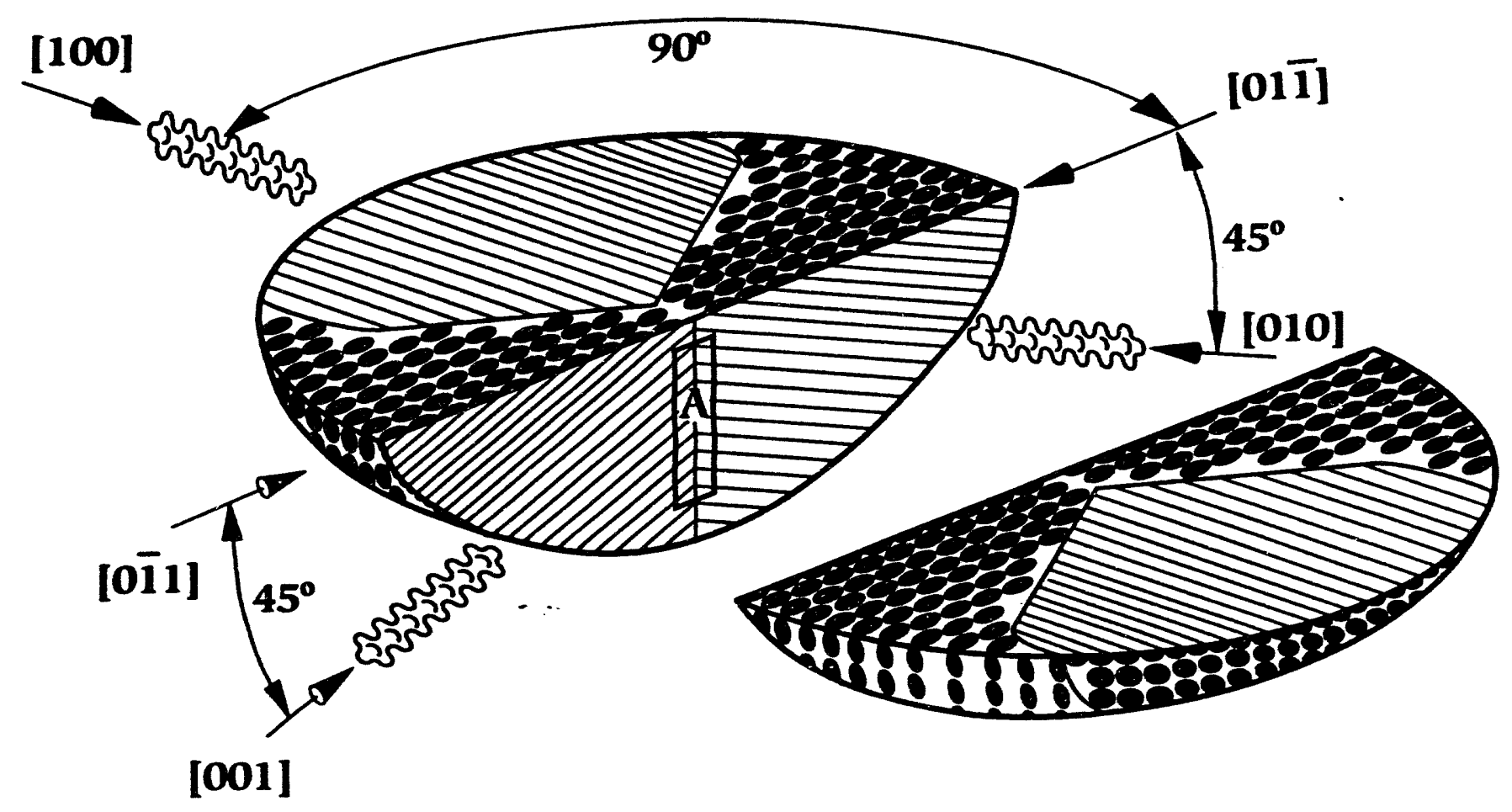

(011) Surface

Gas Tun rsten Arc Weld

Figure 44

Three-dimensional Reconstruction Identifying

High Magnificalton Areas 


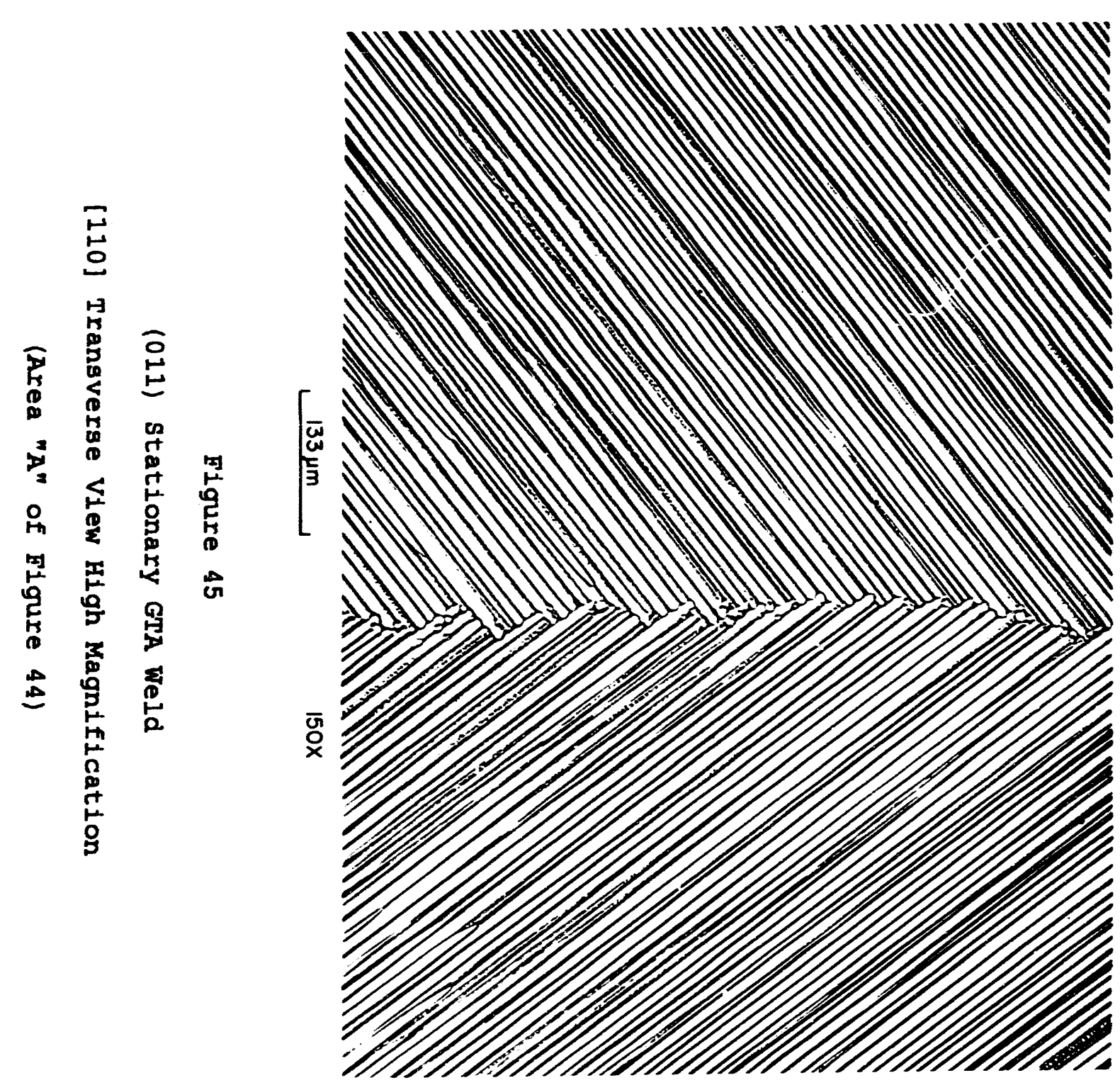


represents the consistency of the [001] and [010] dendrite growths meeting along the centerline of the weld pool. Dendrites match in both directions and demonstrates the symmetry of the microstructure as well as the consistency in comparative growth rates of the two halves.

Sectioning Along the [111] Direction: The GTA stationary welding configuration for the (011) surface and the sectioning for the [111] direction cross-sectional view are schematically represented in Figure 46. The three-dimensional reconstruction and accompanying photomacrographs appear in Figure 47 . The surface view is as described previously for the [100], [010], and [001] dendritic growths. The [111] transverse view represents dendrites that grew at an angle to the cut and met at the weld center (Figure 47c). Figure $47 \mathrm{~b}$ shows the surface view as discussed previously. Figure 47 a shows the three-dimensional reconstruction with the angles at which dendritic growth occurred illustrated.

The higher-magnification photomicrograph area "A" is indicated on Figure 48 . This area is microstructurally represented in Figure 49. It shows that the dendritic cells were elongated instead of circular and that the dendrites met at the approximate center of the weld pool. This microstructure suggests that dendritic growth did not occur perpendicular to the view, but at an angle to the view. 


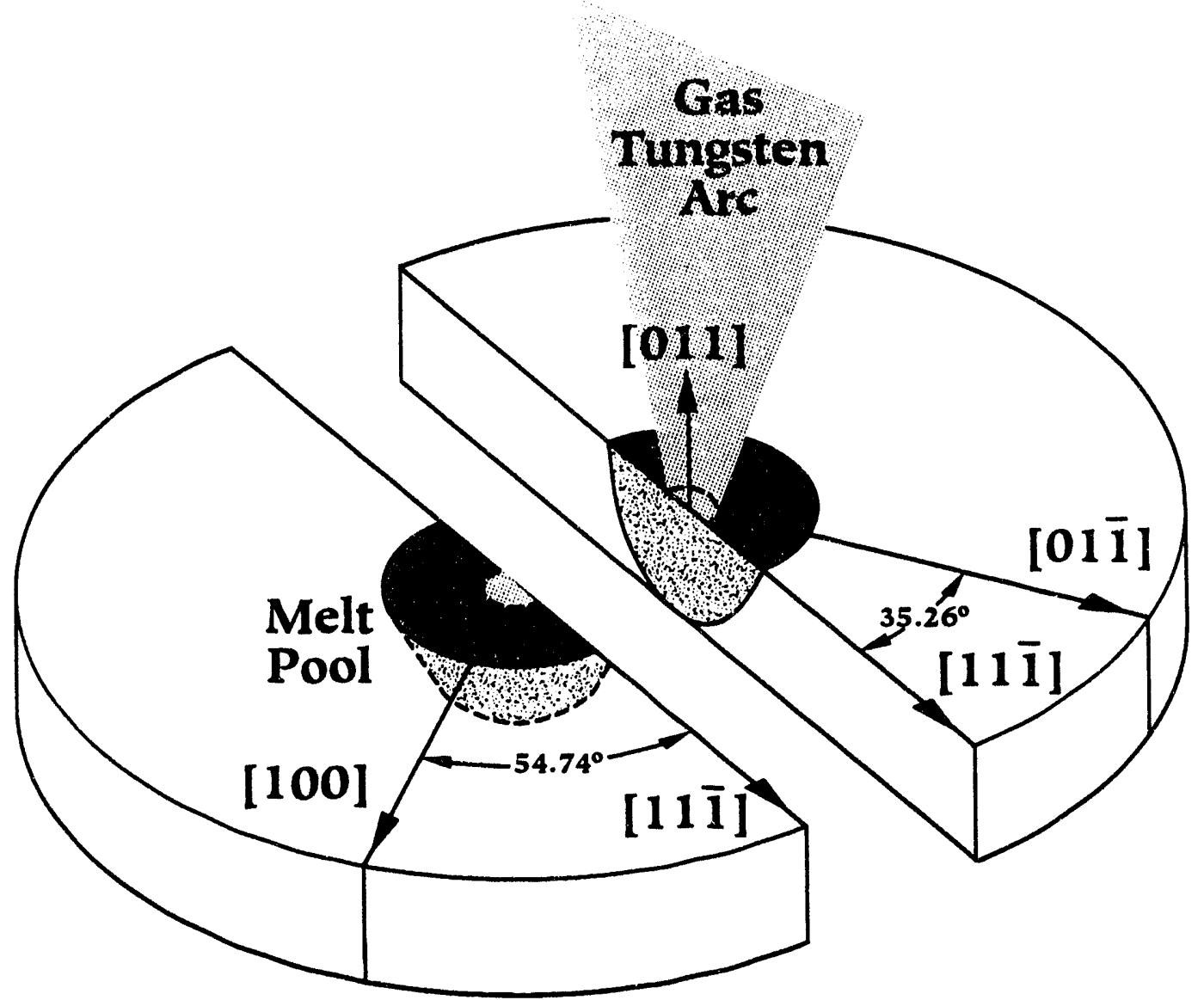

Stainless Steel Single Crystal (011) Surface

Figure 46

Schematic for (011) Surface [111] Direction

Sectioning and Welding Configuration 


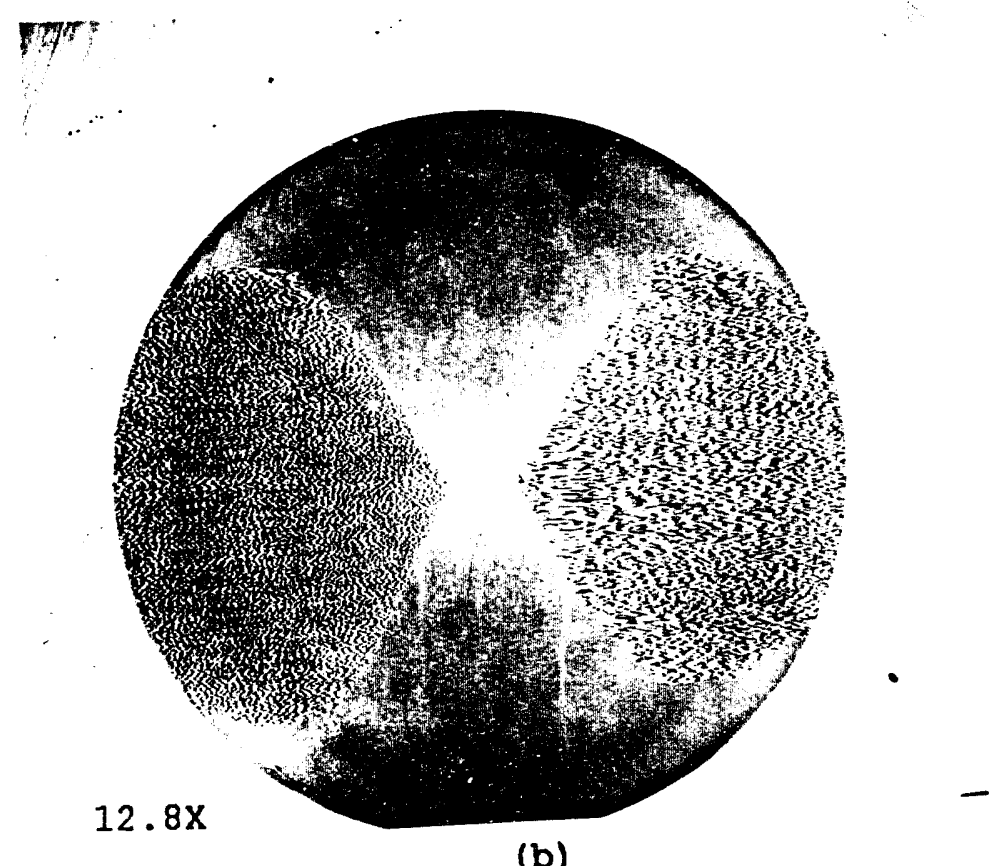

(b)

face View GTA Stationary Weld Top Surface

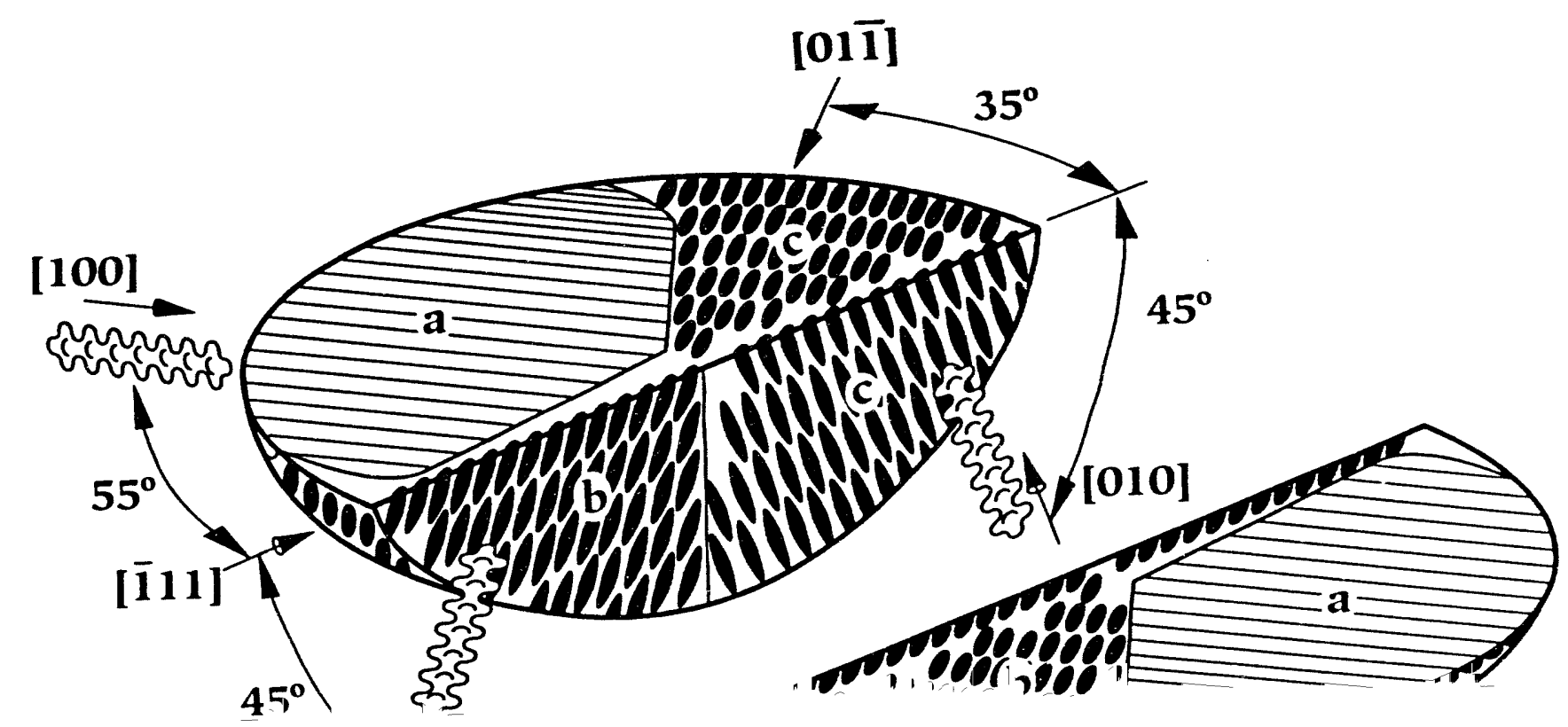




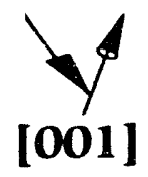

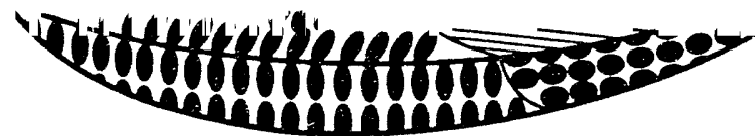

(011) Surface

Gas Tungsten Arc Weld

(a)

Thrae-dimensional Reconstruction of the Resultant Microstructure

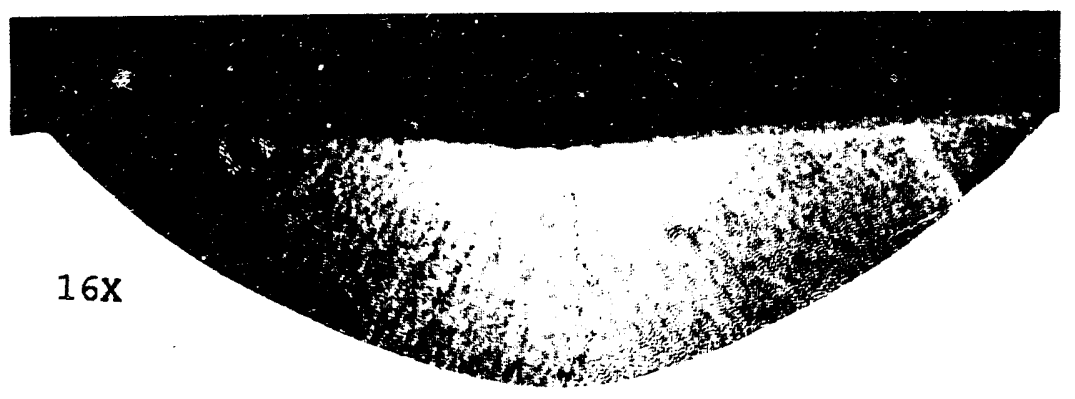

(c)

[111] Direction Transverse View (110) Plane

GTA stationary Weld

Figure 47

Three-dimensional Reconstruction of 11) Plane [111] Direction

Stationary GTA Weld with Accompanying Photomacrographs 


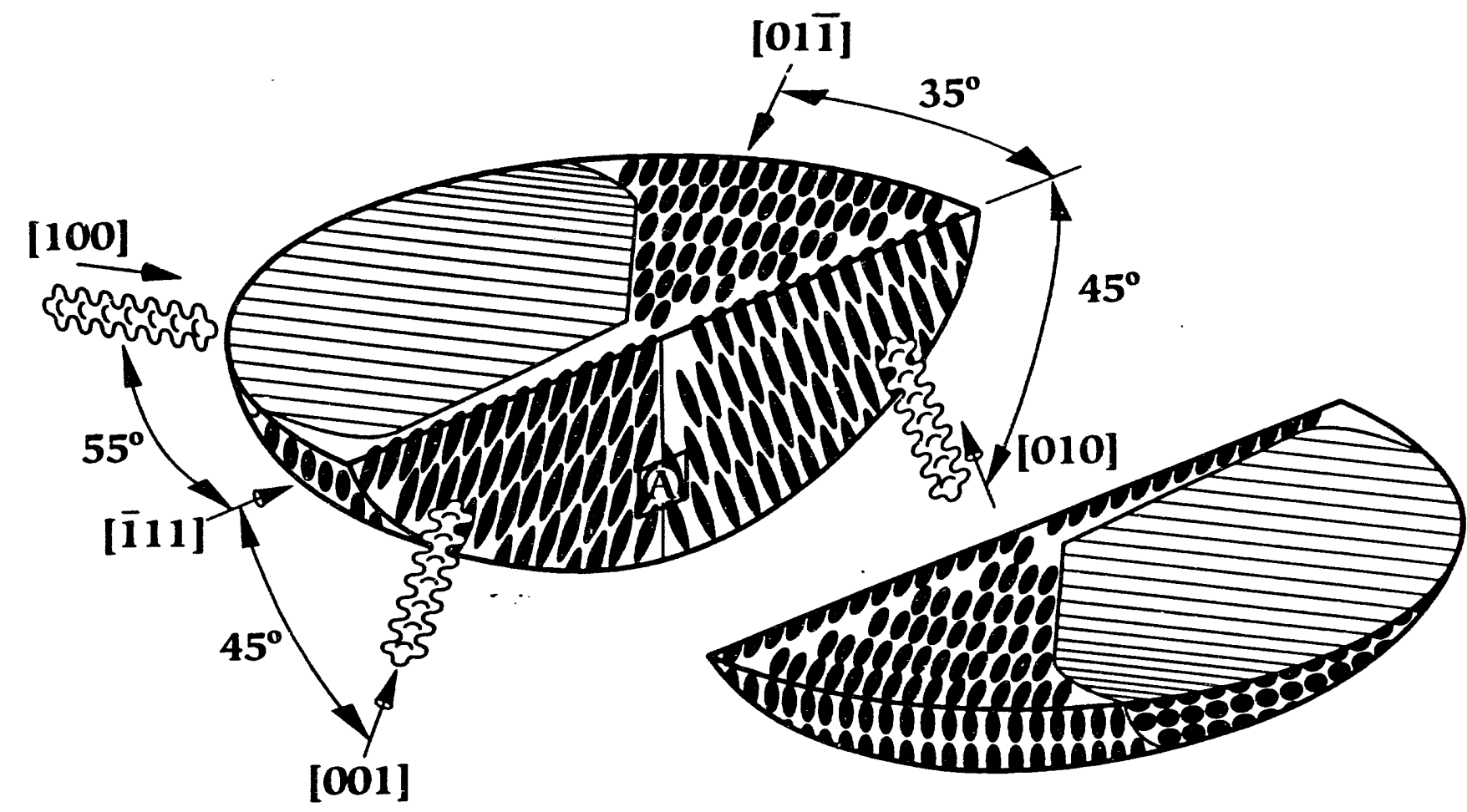

(011) Surface

Gas Tungsten Arc Weld

Figure 48

Three-dimensional Reconstruction

Identifying High Magnification Areas 


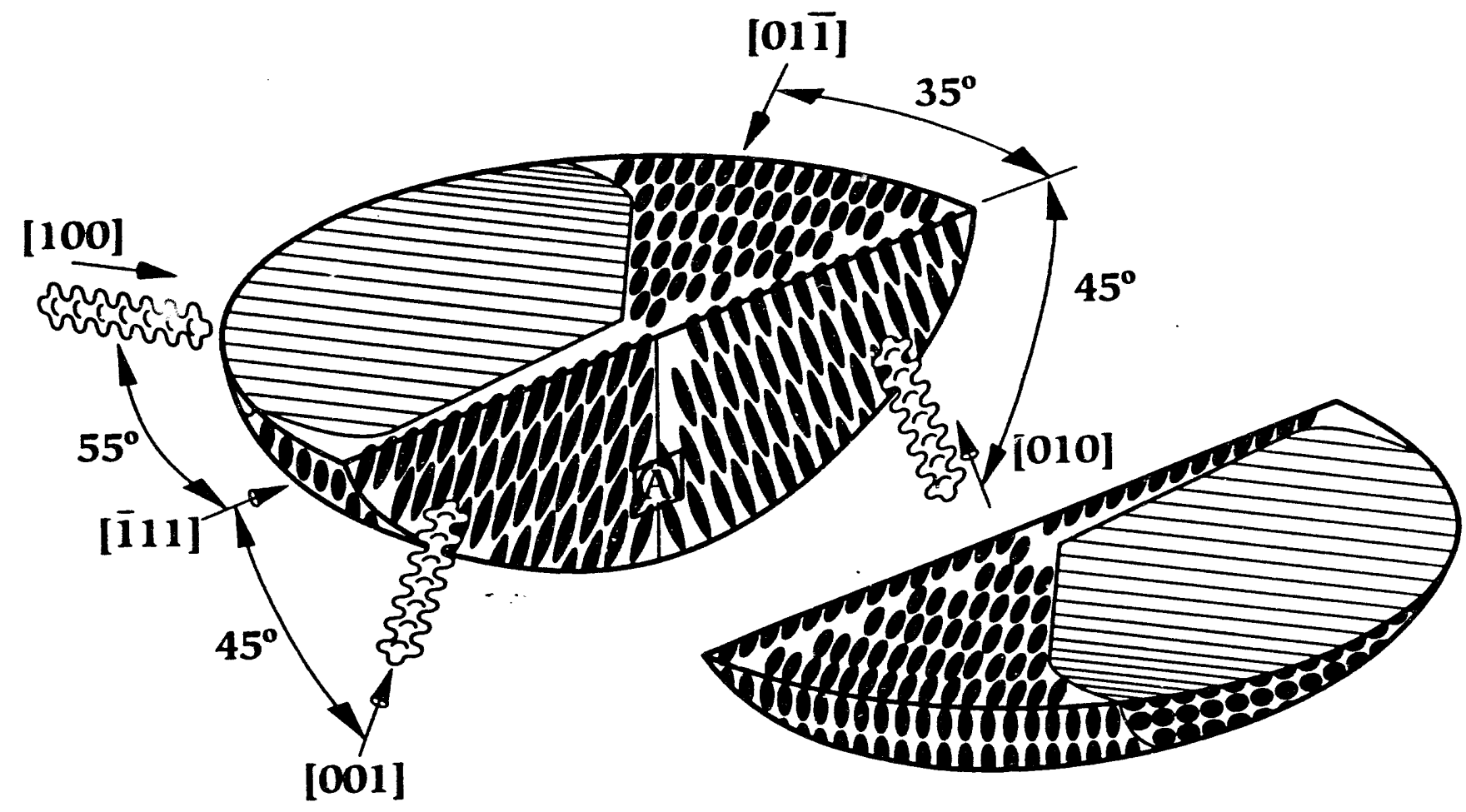

(011) Surface

Gas Tungsten Arc Weld

F1gure 48

Three-dimensional Reconstruction

Identifying High Magnification Areas 


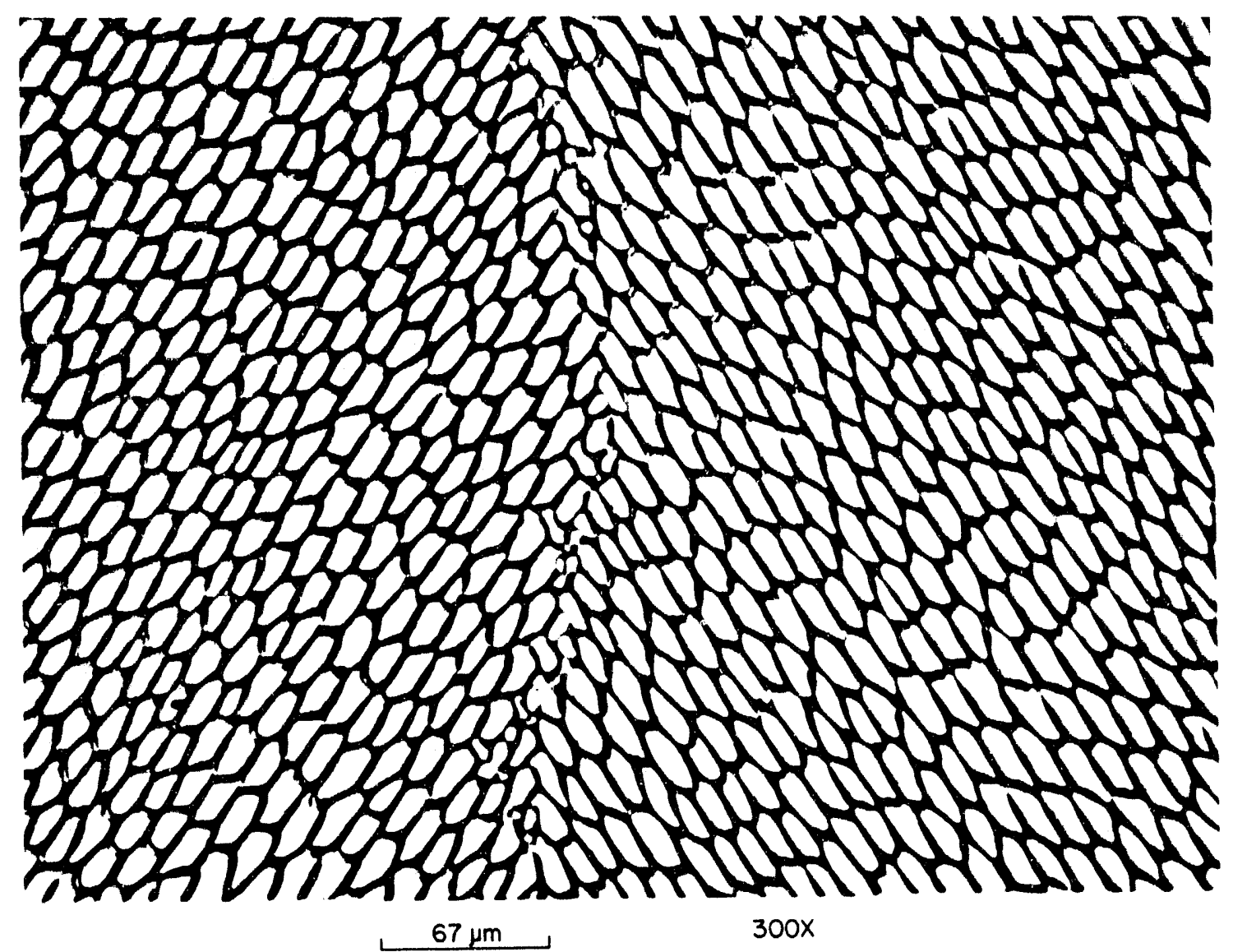

Figure 49

(011) stationary GTA Weld

[111] Transverse View High Magnification

(Area "A" of Figure 48) 
Summary of (011) Orientation

The previous discussion on the solidification of the (011) plane used the surface "in-depth' views as well as the three primary-direction cross-sectional views to determine the solidification pattern three-dimensionally. It was determined and best illustrated in Figure 43a that solidification proceeded in areas "a" along the [100] directions. Simultaneously, solidification occurred in areas " $b$ " along the [001] direction from the bottom and the sides of the weld at an angle of $45^{\circ}$. In areas " $c$ " dendritic growth was in the [001] direction at an angle of $45^{\circ}$ from the (011) surface. Dendrites growing along each of these two directions met along the centerline of the weld.

\section{(111) Crystallographic orientation}

Crystallographic projections for the (111) surface appear in Figure 50 [33]. The preferred dendritic growth directions are denoted by the rectangular areas. These directions are $120^{\circ}$ apart, and the dendrites grow upward at an angle of $55^{\circ}$ from a perpendicular to the surface [34]. This shows that there is a three fold symmetry involved in this crystallographic orientation. From the surface "in-depth" views and the [111] transverse view combined with the stereographic projection, it was possible to form the three-dimensional reconstruction of the 


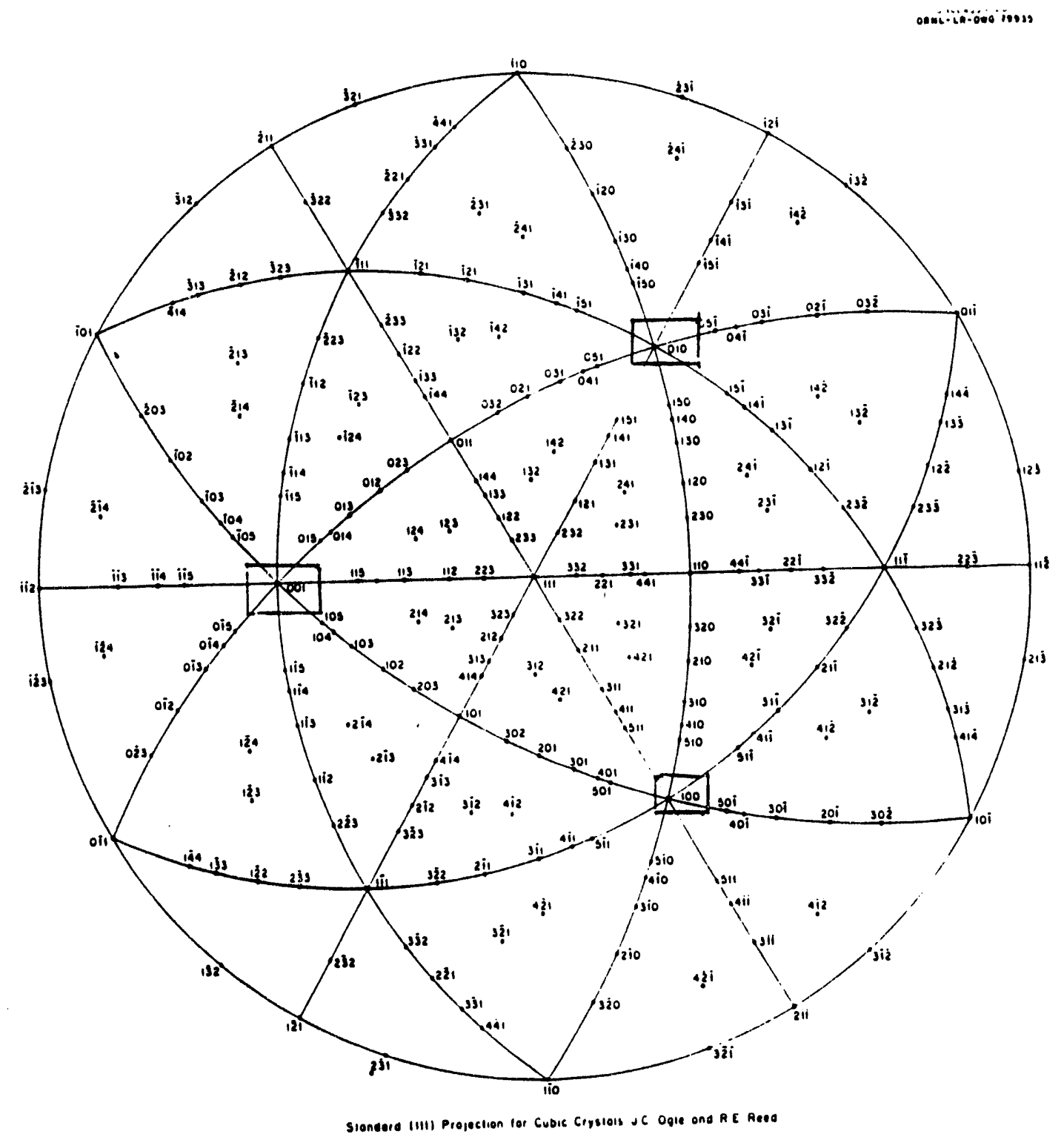

Figure 50

Stereographic Projection for the (111) Plane 
resulting microstructure for a GTA stationary weld made on the (111) crystallographic orientation.

Sectioning Along the [111] Direction: The welding configuration for the (111) plane is represented along with the sectioning configuration for the [111] primary direction of this crystallography in Figure 51. Figure 52 depicts the threedimensional reconstruction for this direction and the relevant photomacrographs. The surface view shows the three fold symmetry of the microstructure. The dendrites all grew at a fixed angle relative to the surface. The [111] transverse view microstructure revealed dendrite growth which was at an angle to the cut. The formation of the cross sectional dendrites were elliptical. To determine the active growth directions, stereographic projections were used. Three $\langle 100\rangle$ primary growth directions in the interior of the crystal form the three quadrants visible noted on the surface view.

Top surface: Figure 53 depicts the schematic of the welding configuration for the (111)-plane-surface views and denotes the primary direction along the periphery. Figure 54 illustrates a three-dimensional reconstruction of the resultant microstructure with applicable photomacrographs. A series of top-surface views appears in Figure 54b,c. The top-surface view shows that all the dendrites grew in an upward direction toward the surface. It is 


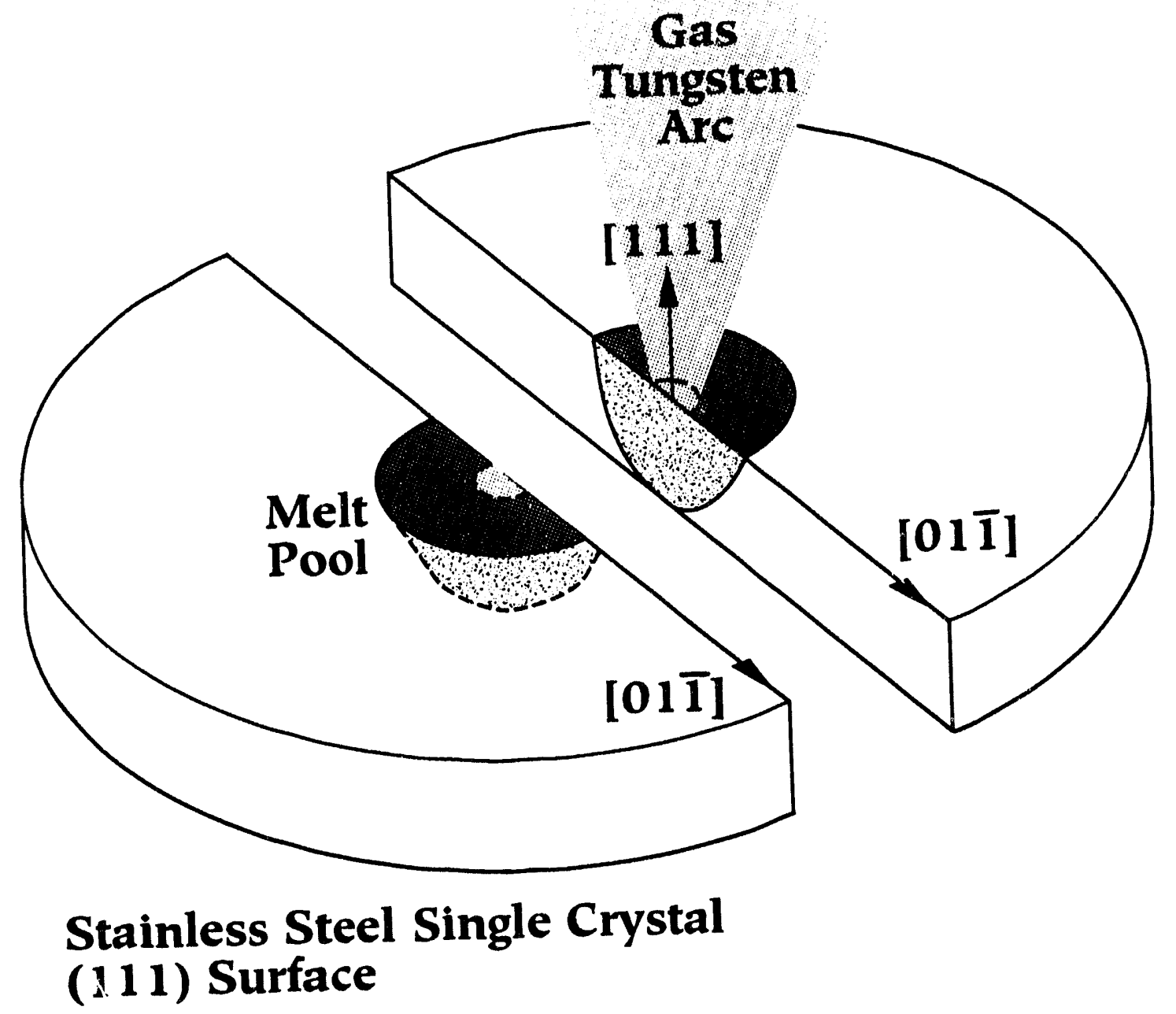

Figure 51

Schematic for (111) Surface [110] Direction

Sectioning and welding Configuration 



\section{$/ 55^{\circ}$ y (111) Surface \\ [001] \\ Gas Tungsten Arc Weld}

(a)

Three-dimensional Reconstruction of the Resultant Microstructure

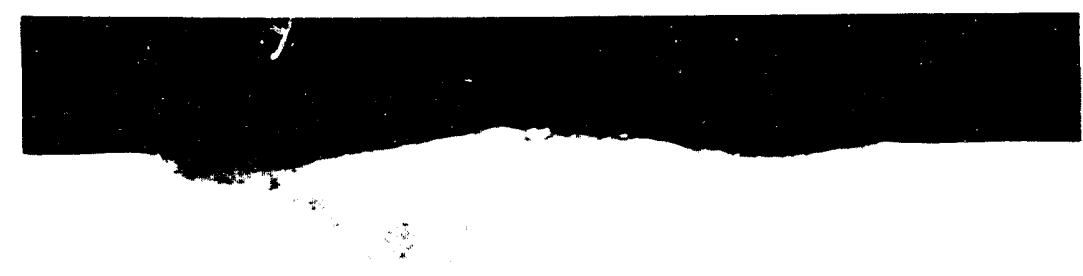

$20 x$

(c)

(111) Surface [110] Direction Stationary GTA Weld

\section{Figure 52}

Three-dimensional Reconstruction of (111) Plane [110] Direction

Stationary GTA Weld with Accompanying Photomacrographs 


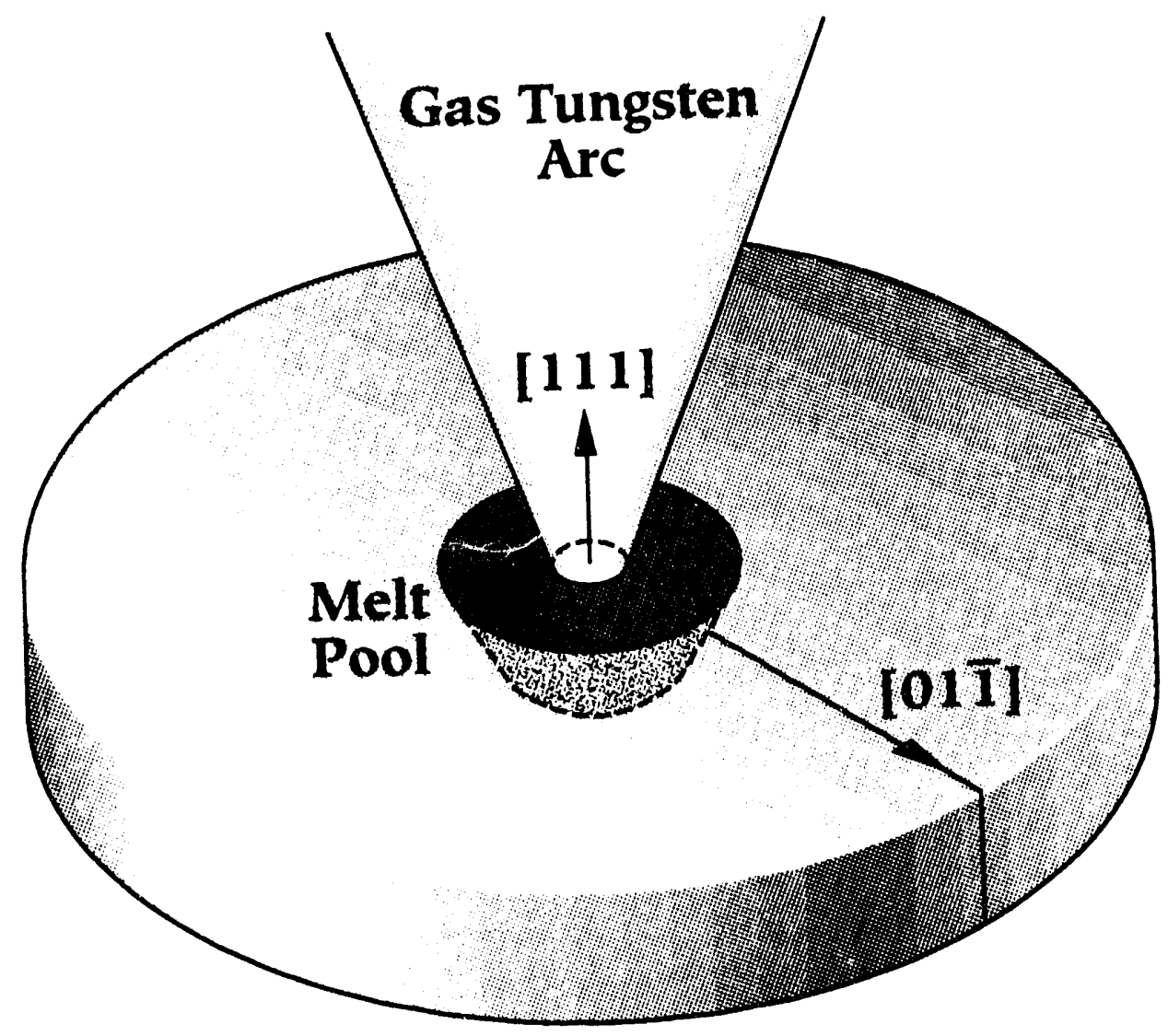

Stainless Steel Single Crystal (111) Surface

Figure 53

Schematic for (111) surface Welding Configuration 


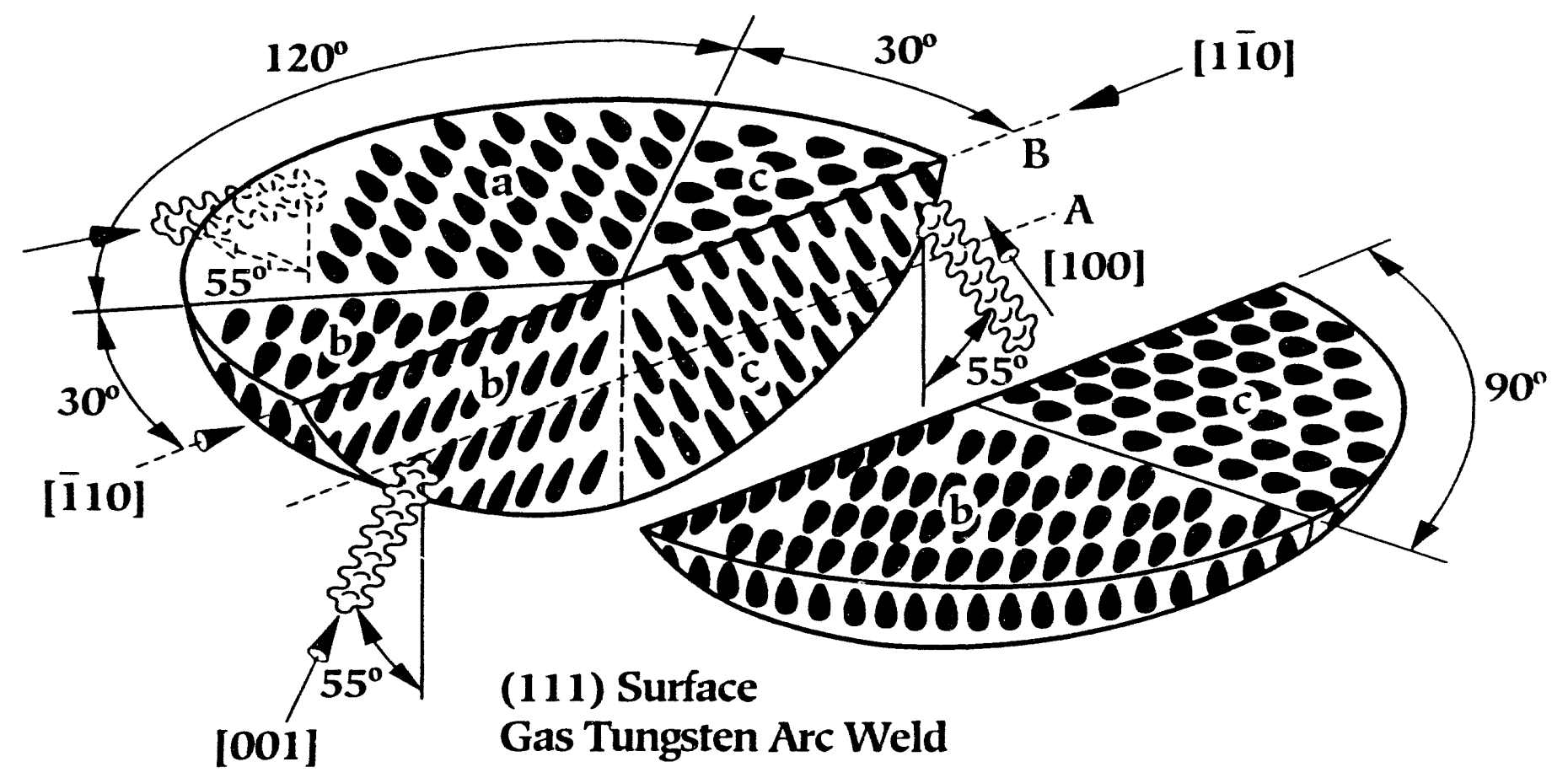

(a)

Three-dimensional Reconstruction of Resultant Microstructure

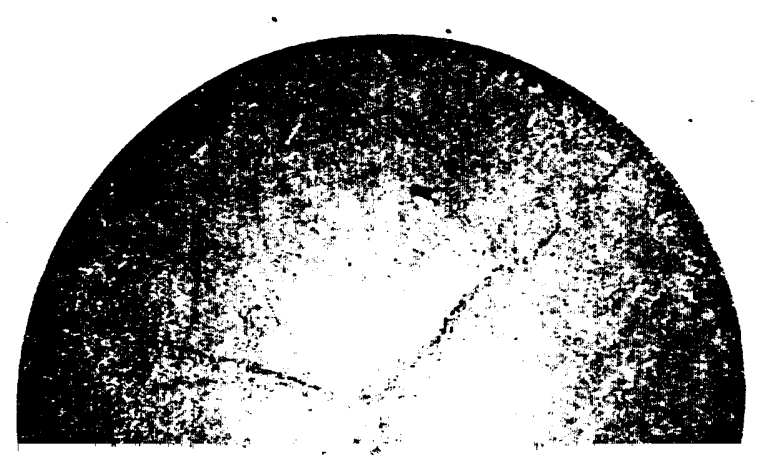


seen that the growth formed a three fold symmetric pattern. The elongated appearance of the dendritic cells, however, indicates they were not growing perpendicular to the surface. Therefore, it can be deduced that growth occurred along the three interior preferred growth directions at an angle to the surface of $55^{\circ}$ as determined by the stereographic information and yielded the three-fold symetry observed in the surface view.

\section{Summary of (111) Orientation}

From the transverse view, the surface "in-depth" views, and the stereographic projections the solidification microstructure for the (111) plane GTA stationary weld was determined and is illustrated in Figure $47 \mathrm{a}$. Solidification occurred simultaneously from the surface, side, and bottom of the weld in areas " $a$ ", "b", and " $c$ " along the three interior preferred growth directions, $[010],[001]$, and $[100]$ respectively. These dendrites met at either the center of the weld or along the $\langle 211\rangle$ family of directions.

\section{Gas Tungsten Arc Rotational Felds}

Rotation of the crystal during GTA welding was used to ascertain if a more-circular weld pool shape and a more-symetric microstructure could be obtained. The solidification patterns 
and resultant microstructures were similar to the stationary GTA welds for the respective planes. This resulted in similar microstructures, thus the three-dimensional reconstruction of the resulting microstructures were also similar. For each plane, (001), (011), and (111), the macrographs for the surface views at various depths, and transverse views of the primary directions, as well as additional microstructural information, are presented.

\section{(001) Crystallographic Orientation}

The photomacrographs for the two primary directions and the surface views are represented along with the accompanying threedimensional reconstruction in Figures 55, 56, and 57. The solidification patterns are the same as described previously in each respective view in the discussion of the (001)-plane stationary welding.

Figure 58 shows the schematic for areas " $A$ ", "B", and "C" for which further microstructural analysis was conducted. Area "A" of Figure 58 is microstructurally represented in Figure 59. symmetry in the microstructure can be seen in the photomacrograph from the mirror image which is represented. The dendritic velocity of each of the favored directions being different allowed for the microstructural development. Area "B" of Figure 58 is represented in Figure 60 . The top surface view demonstrates the symmetry of the weld which exhibits a well- 


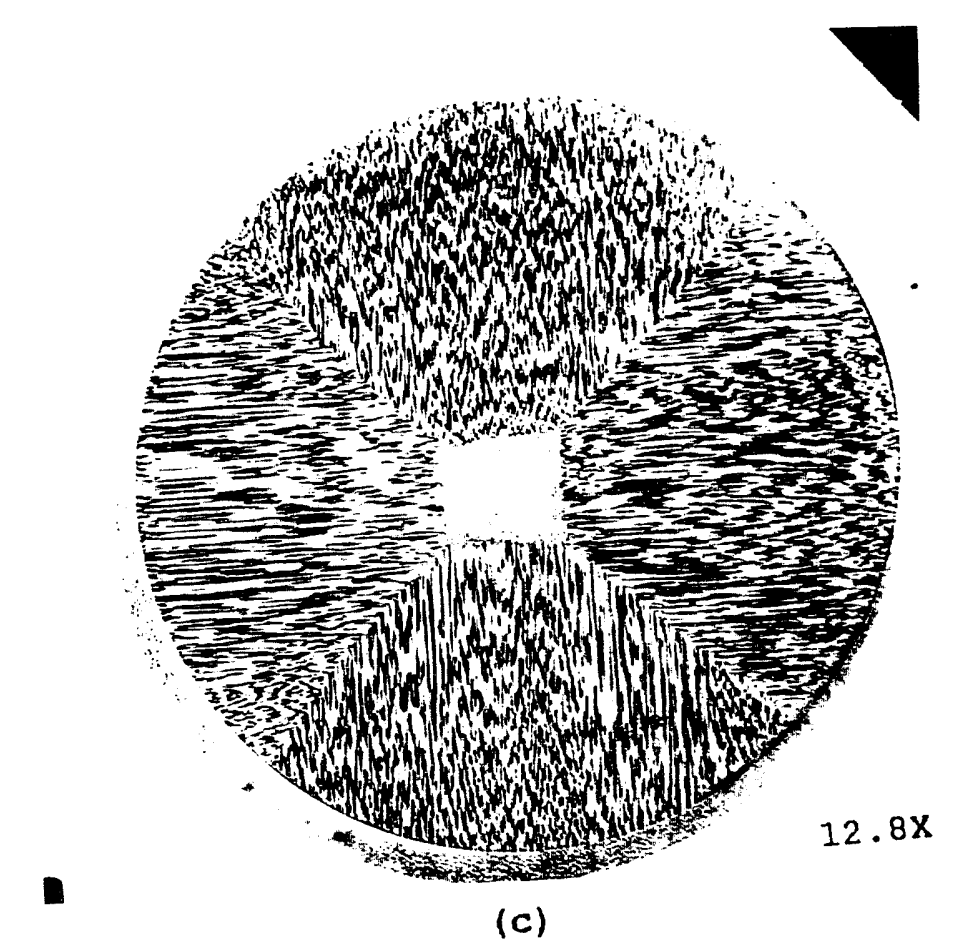

(001) Surface View GTA Rotational Top of weld

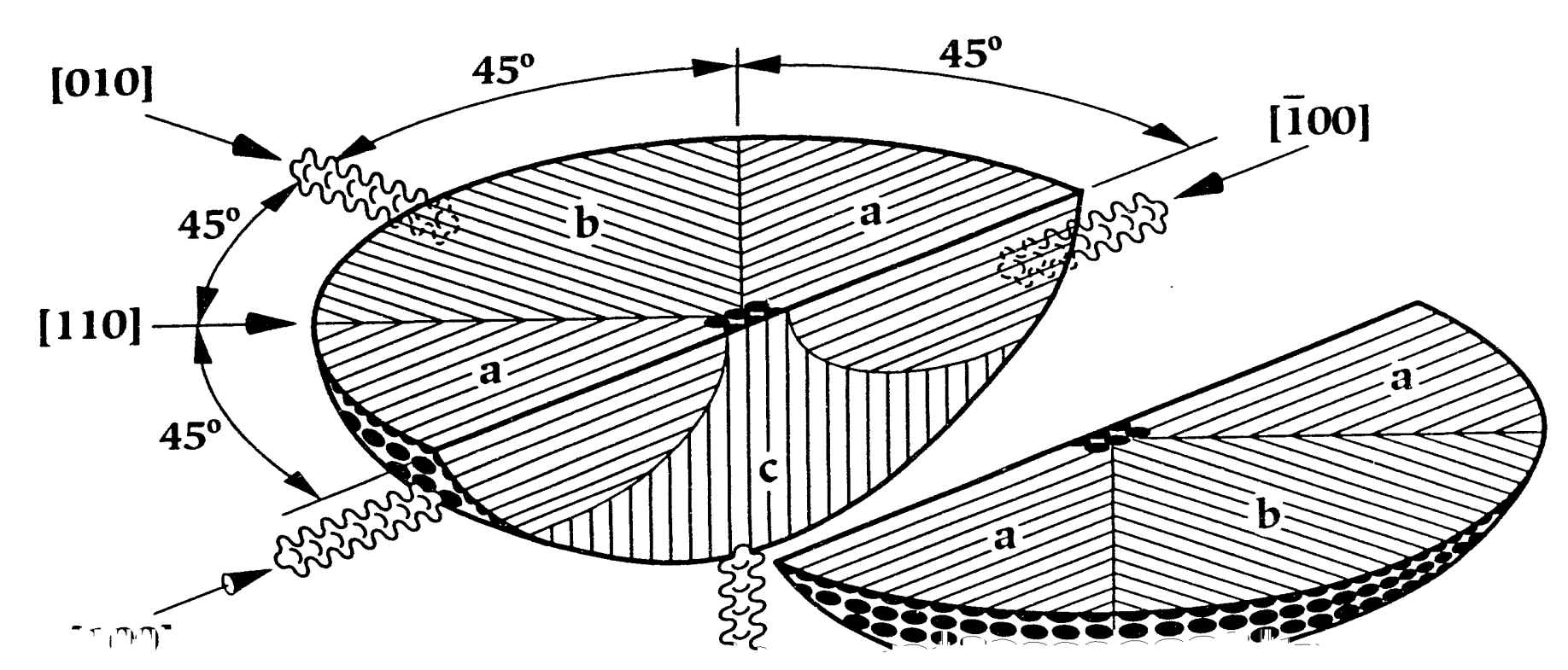




\section{(001) Surface}

Gas Tungsten Arc Weld

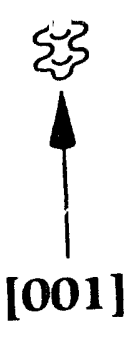

(a)

Three-dimensional Reconstruction of the Resultant Microstructure

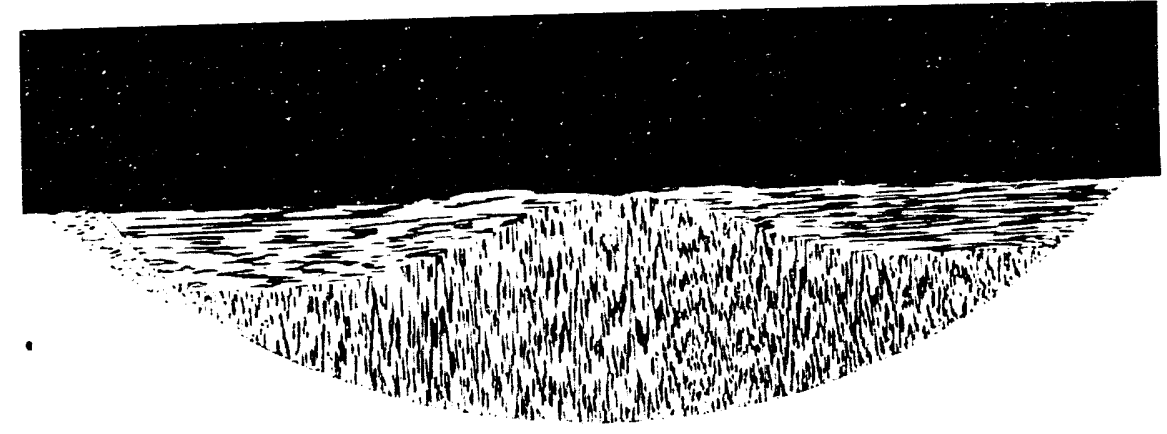

$16 \mathrm{X}$

(b)

Transverse View GTA Rotational Weld [100] Direction

Figure 55

Three-dimensional Reconstruction (001) Plane [100] Direction

Rotational GTA Weld with Accompanying Photomacrographs 


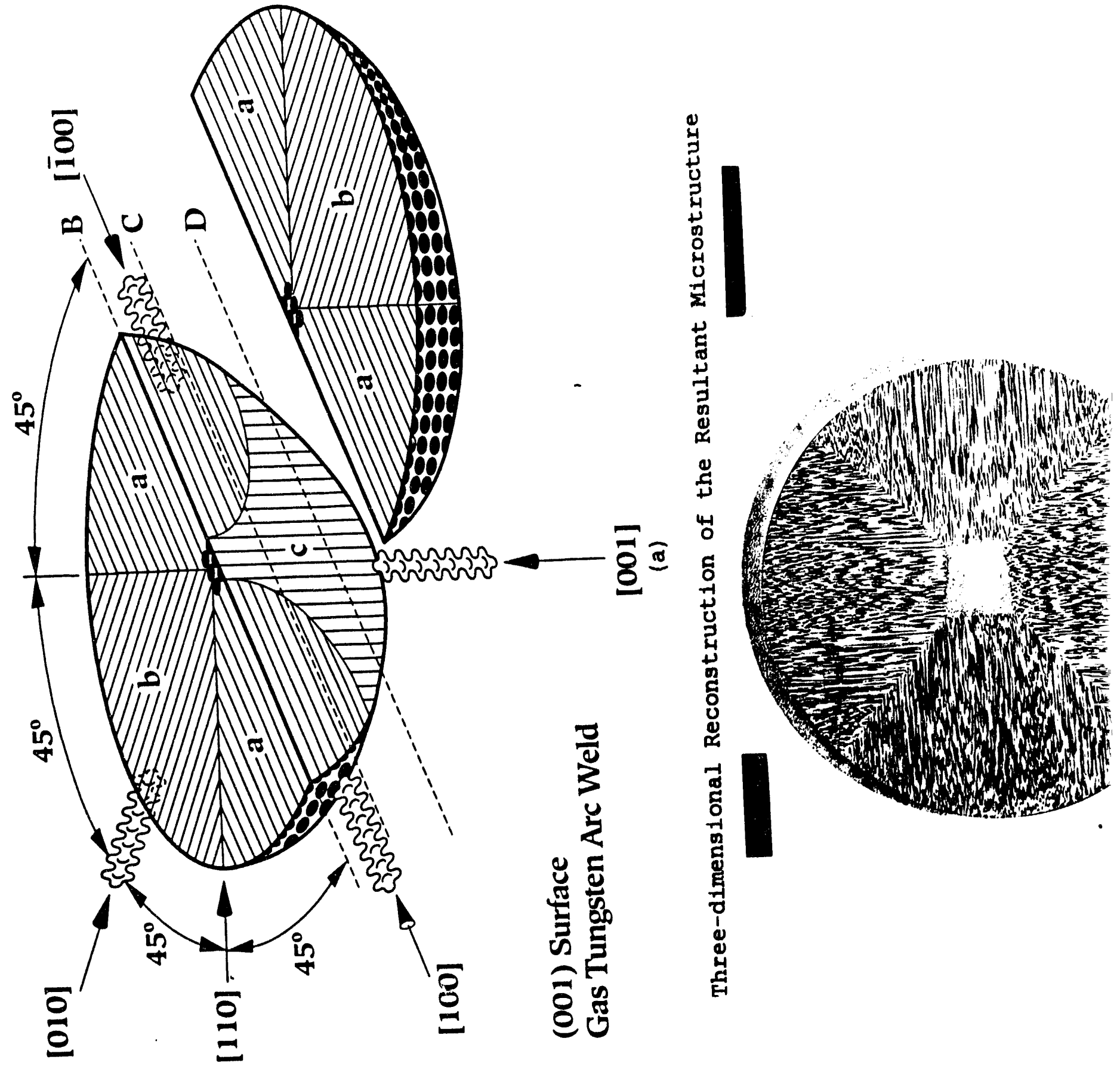




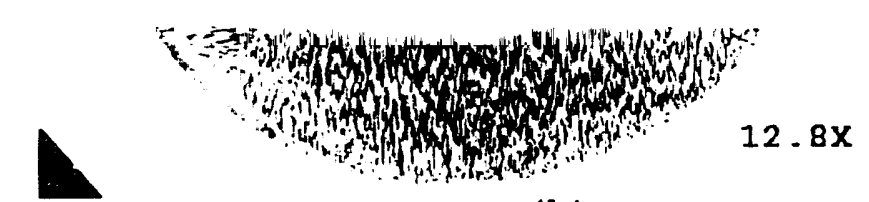

(b)

(001) Surface View GTA Rotational Top of Weld

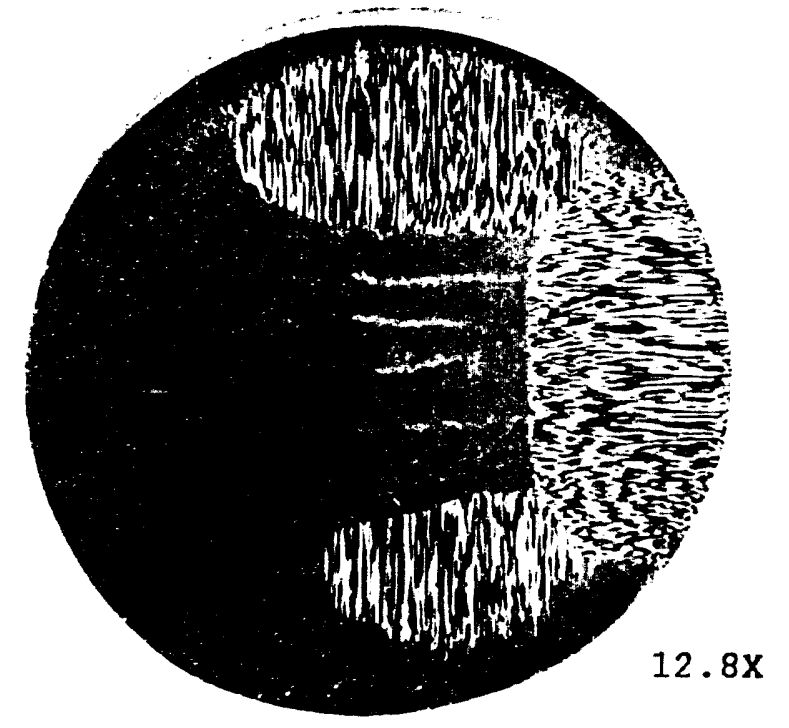

(c)

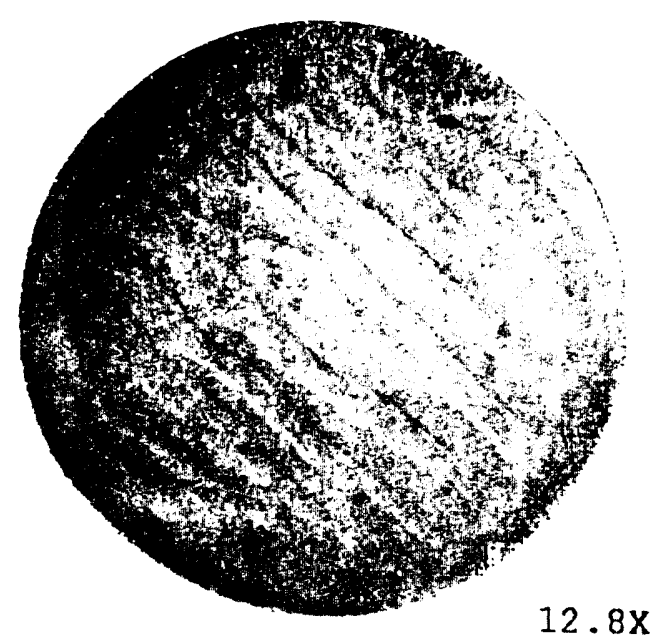

(d)

(001) Surface View GTA Rotational Weld Deeper into weld

(001) Surface View GTA Rotational Weld Complete Coverage of [001] Dendrites Figure 56

Three-dimensional Reconstruction (001) Plane Surface Rotational GTA Weld with Accompanying Photomacrographs 

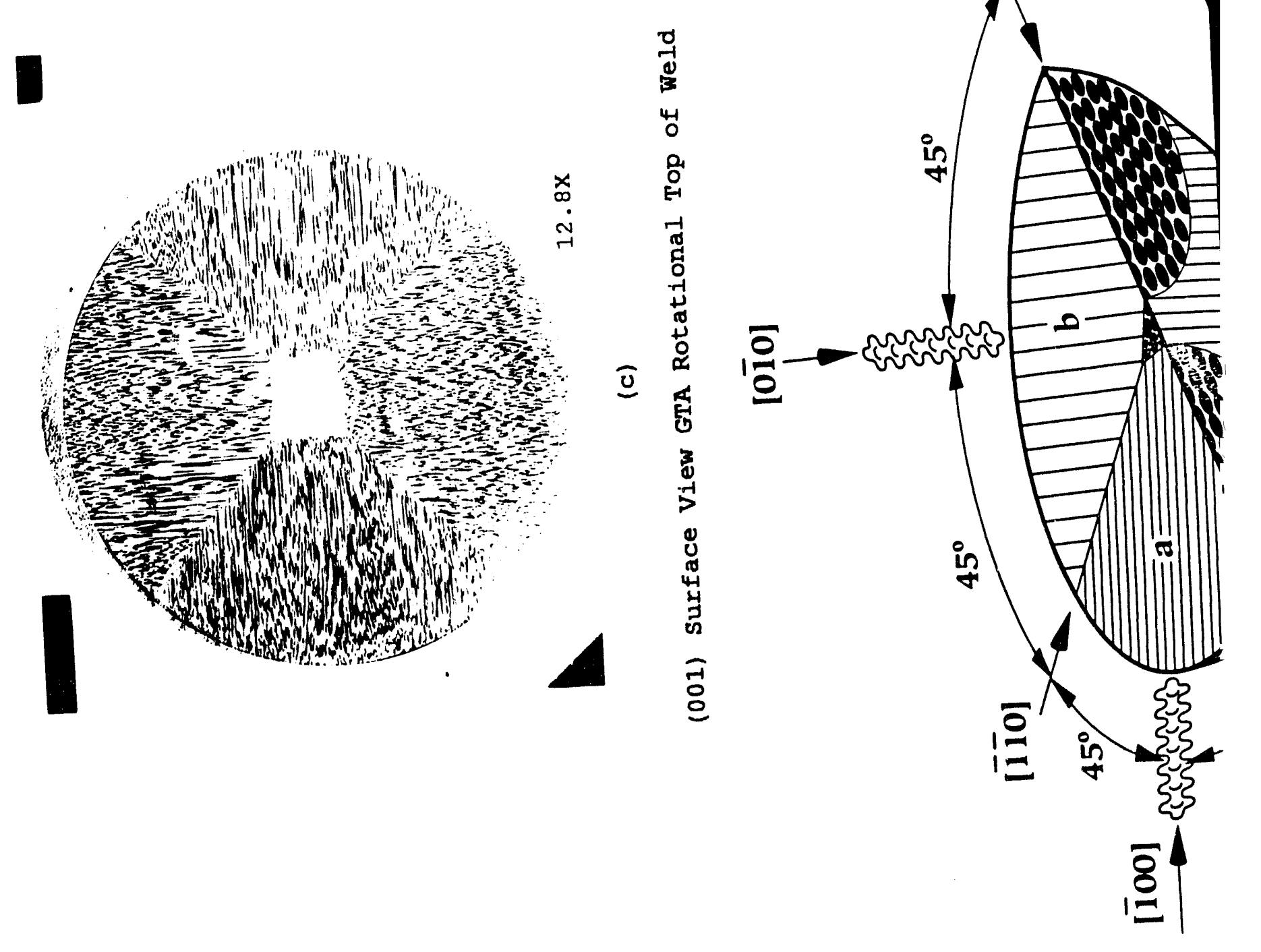
(001) Surface

(001) Surface
Gas Tungsten Arc Weld

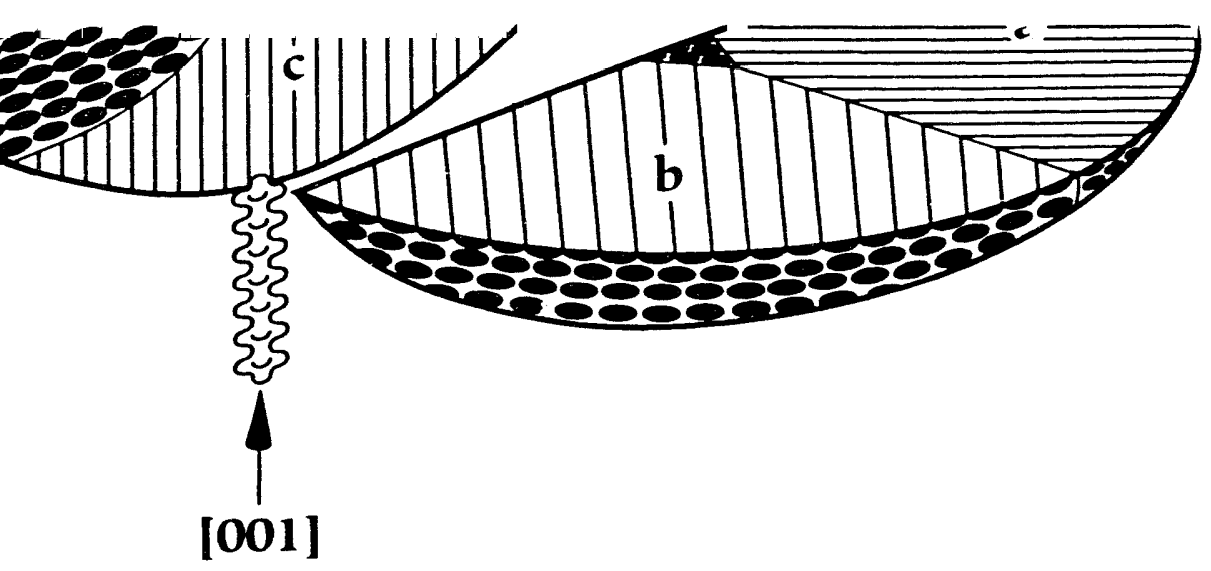

(a)

Three-dimensional Reconstruction of the Resultant Microstructure

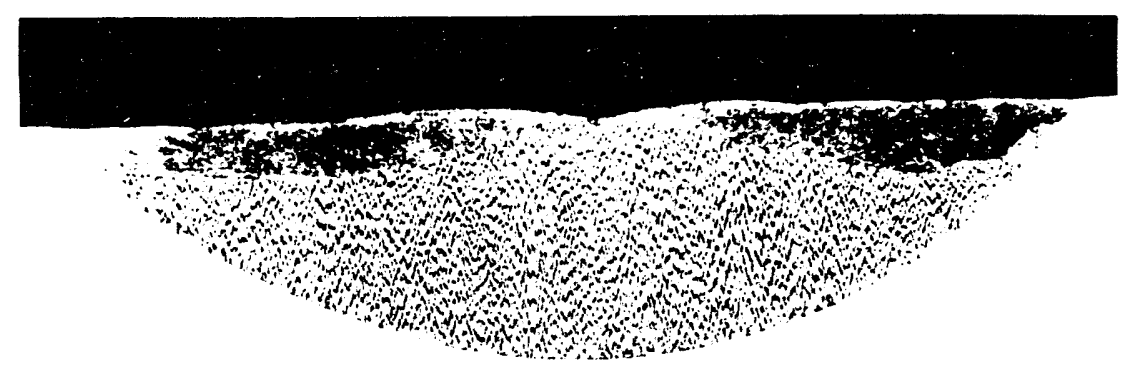

$16 \mathrm{x}$

(b)

Transverse View GTA Rotational Weld [110] Direction

Figure 57

Three-dimensional Reconstruction (001) Plane [110] Direction

Rotational GTA Weld with Accompanying Photomacrographs 


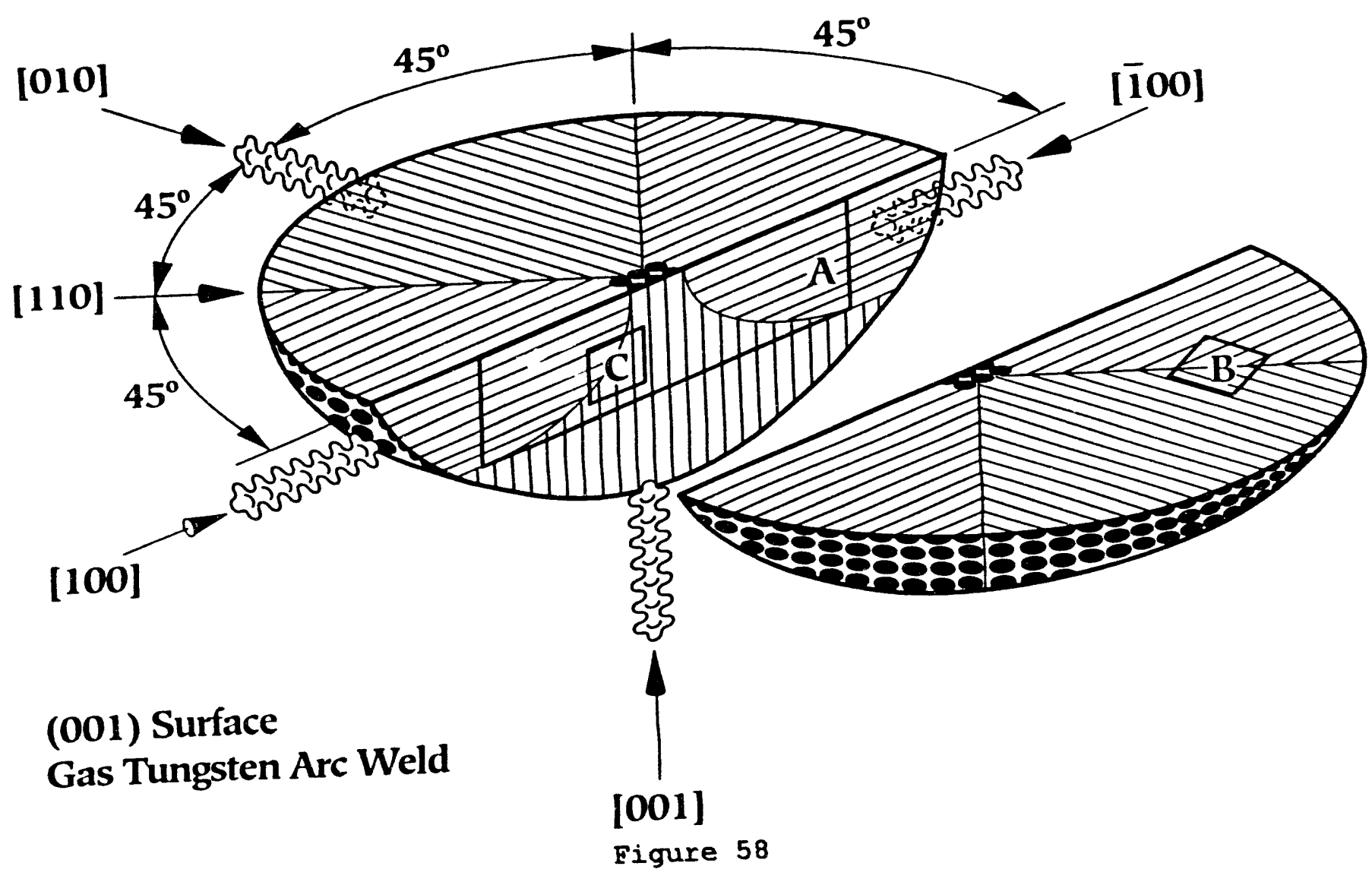

Three-dimensional Reconstruction

Identifying High Magnification Areas 


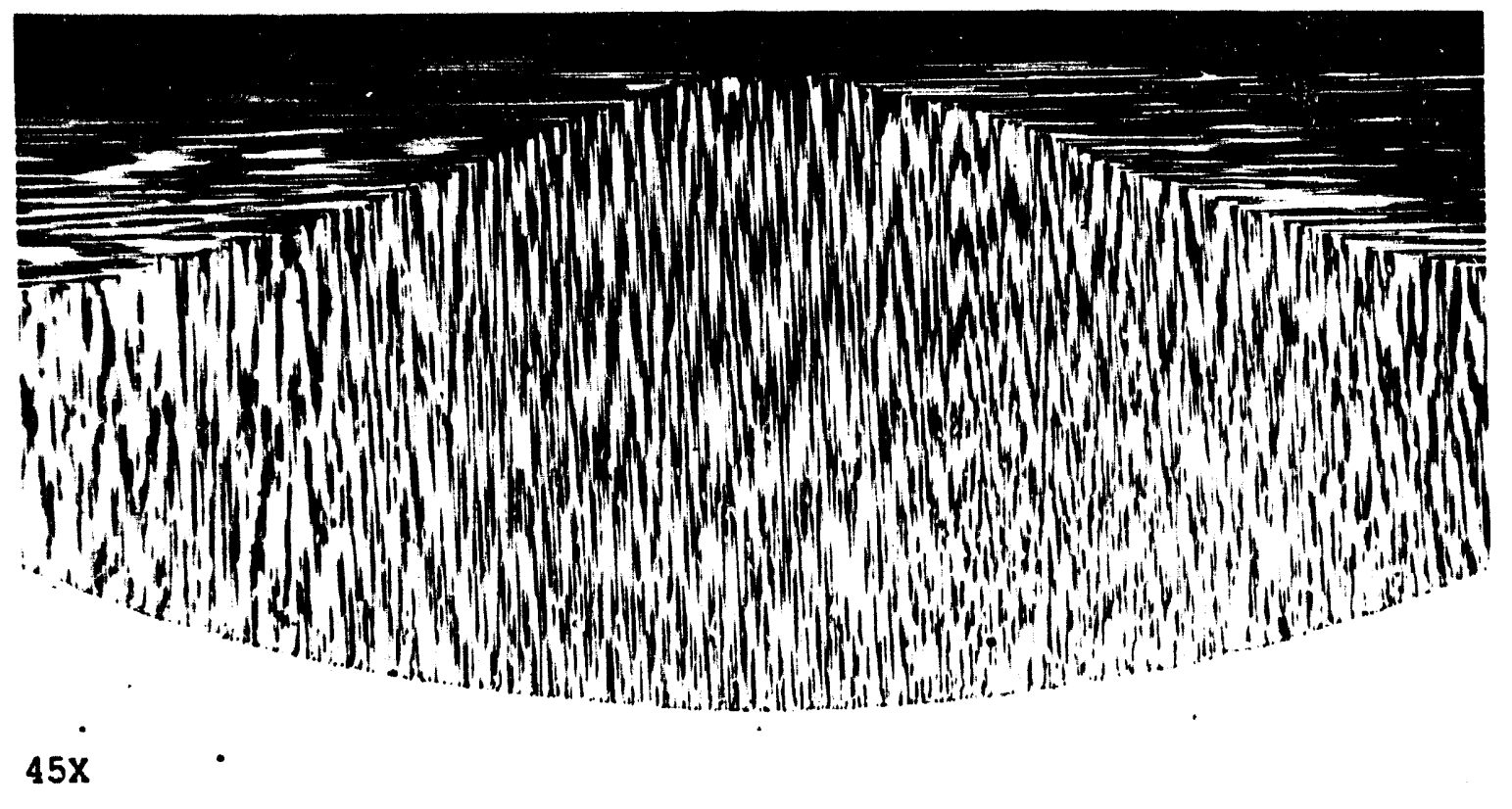

Figure 59

Transverge View GTA Rotationel Weld [100] Direction

(Area "A" of Figure 58) 


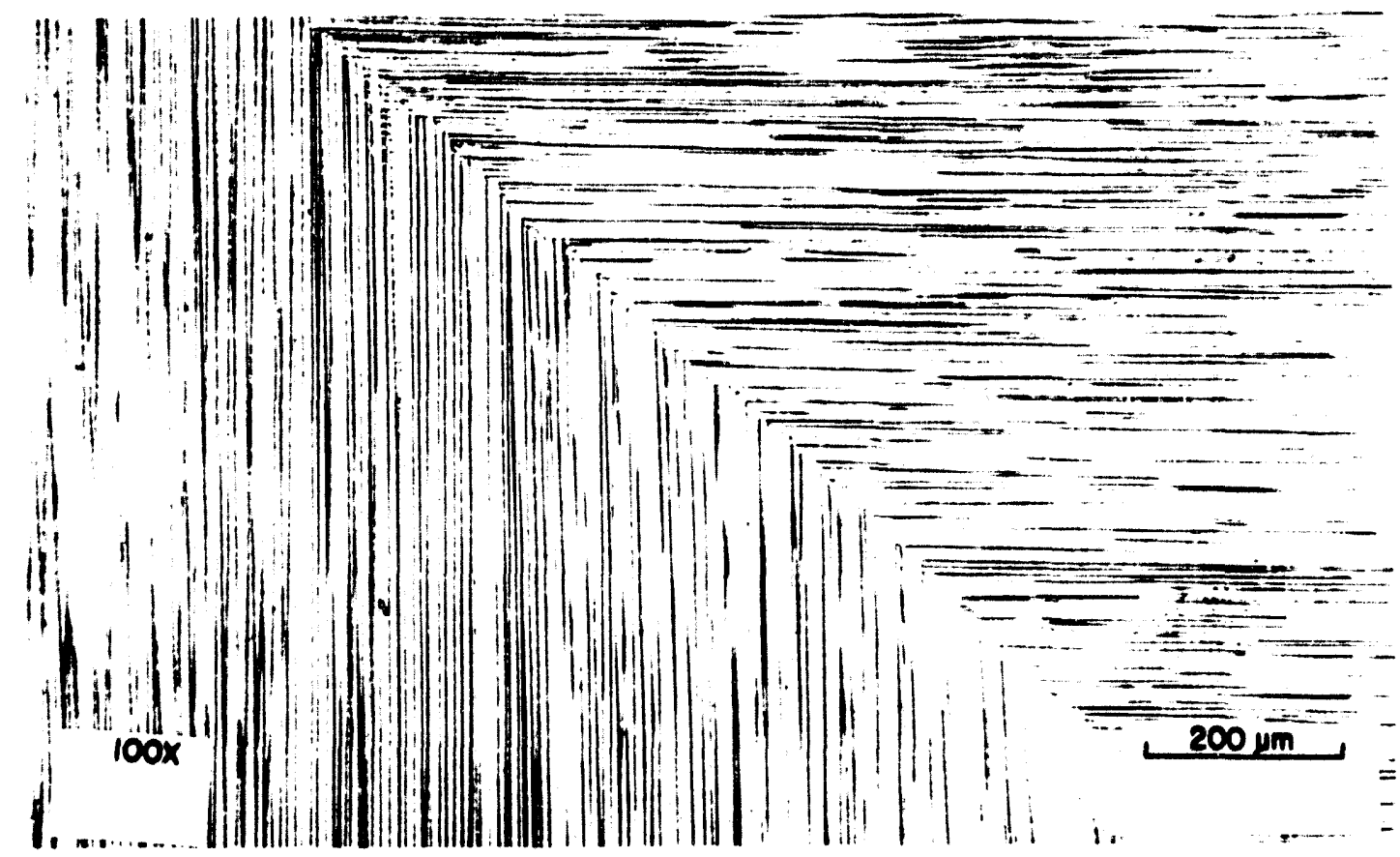

Figure 60

(001) Surface View GTA Rotational Weld Dendrites Along [110] (Area "B" of Figure 58) 
defined meeting of the [100] and [010] dendrites. Area "C" of Figure 58 is represented in Figure 61. Dendritic growth indicating the solidification patterns of the sides of the weld was also evident in the photomicrograph. All of these presented a comparatively uniform growth rate forming the symmetry of each section.

An interesting solidification phenomena appeared in a rotational weld. It was possible to observe the dendrites, surface ripples, and slip lines on the weld surface in the aswelded condition. Areas "A", "B", "C", "D", ${ }^{E n}$, and $" F^{n}$ in whlch photomicrographs were taken to represent the microstructure are presented in Figure 62. The ripples, dendrites, and slip lines of areas " $A$ " and " $B$ " from Figure 62 can be seen in Figures 63 and 64 . The dark sections represent the peaks of the surface ripples. The lines which proceed across the micrograph are the [100] dendrites. Finally, faint lines at a angle $45^{\circ}$ from the dendrites represent the $\{111\} /[110]$ slip lines. Figures 65 and 66 show the dendritic growth in the [100] and [010] directions are shown microstructurally from areas " $C$ " and " $D$ " of Figure 62 . The [100] and [010] dendrites are visible with the slip lines $45^{\circ}$ from these dendrites. The shadowed arcs on the photomicrographs represent the ripples on the surface of the weld. Area "E" of Figure 62 is shown in Figure 67, 1llustrating the [100] dendrites and the $\{111\} /\langle 110\rangle$ slip lines at a $45^{\circ}$ angle from the dendrites 


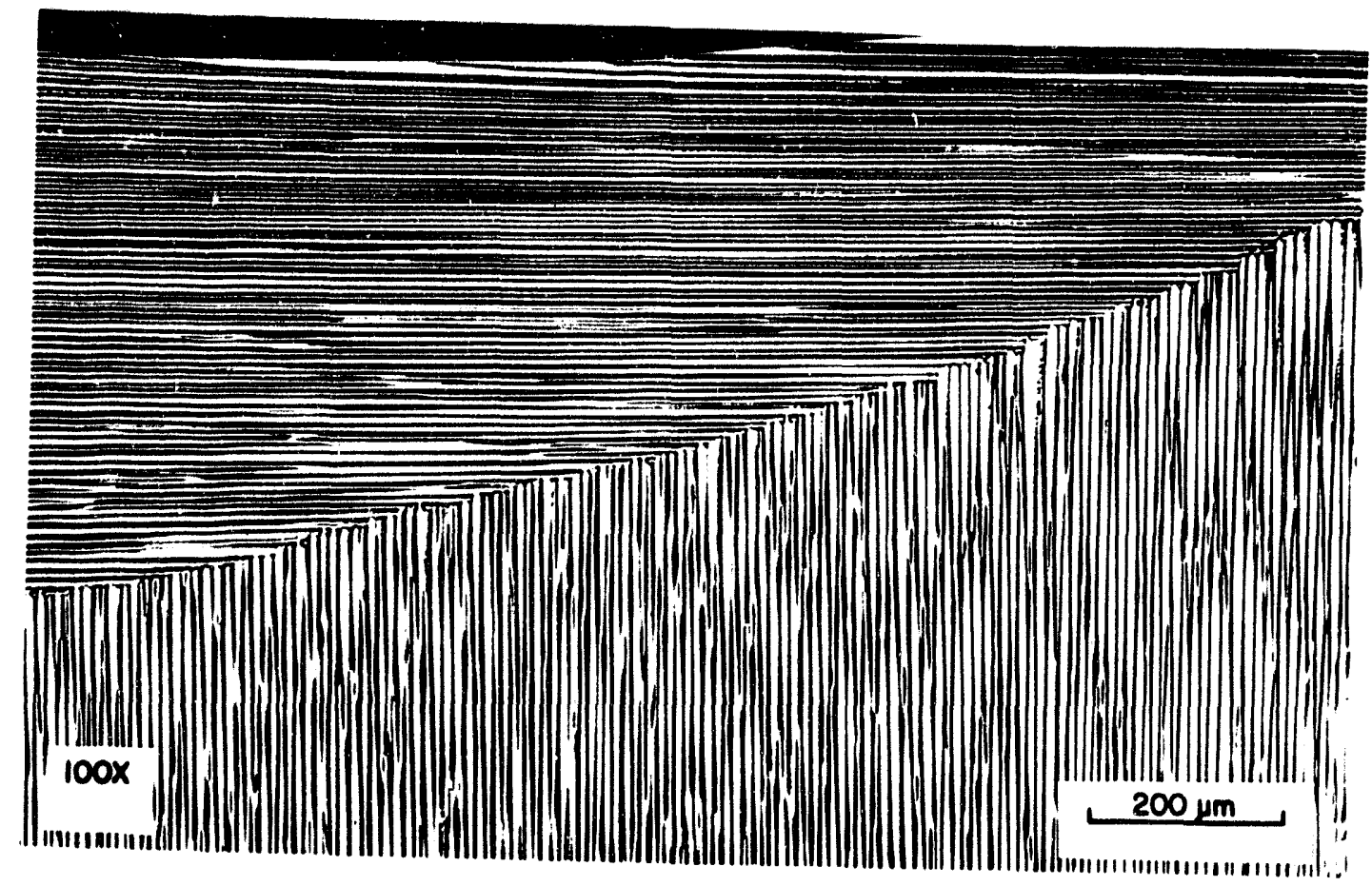

Figure 61

Transverse View [100] Direction GTA Rotational Weld

High Magnification of the side section

(Area "C" of Figure 58) 


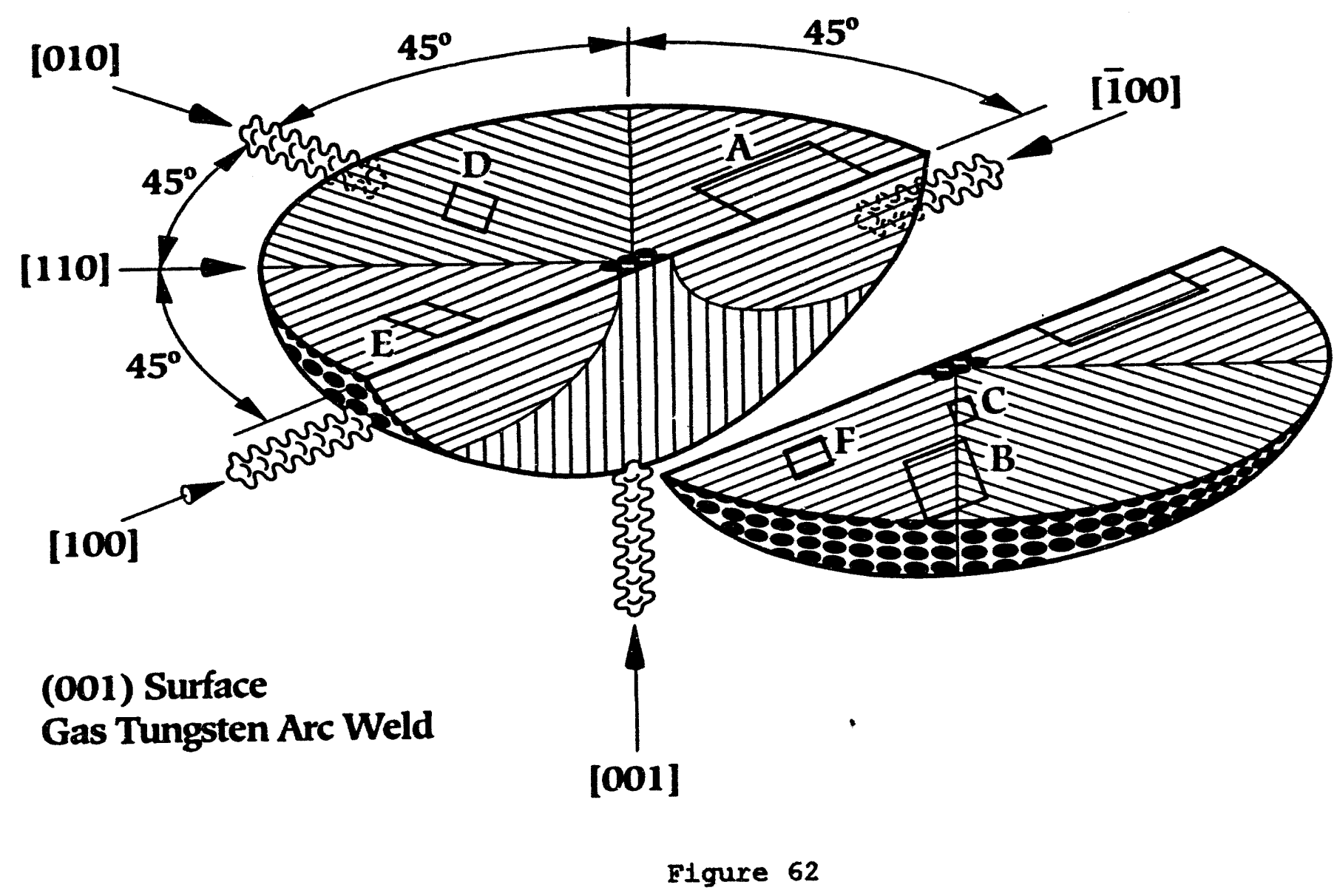

Three-dimensional Reconstruction Identifying

Micrographed Areas 


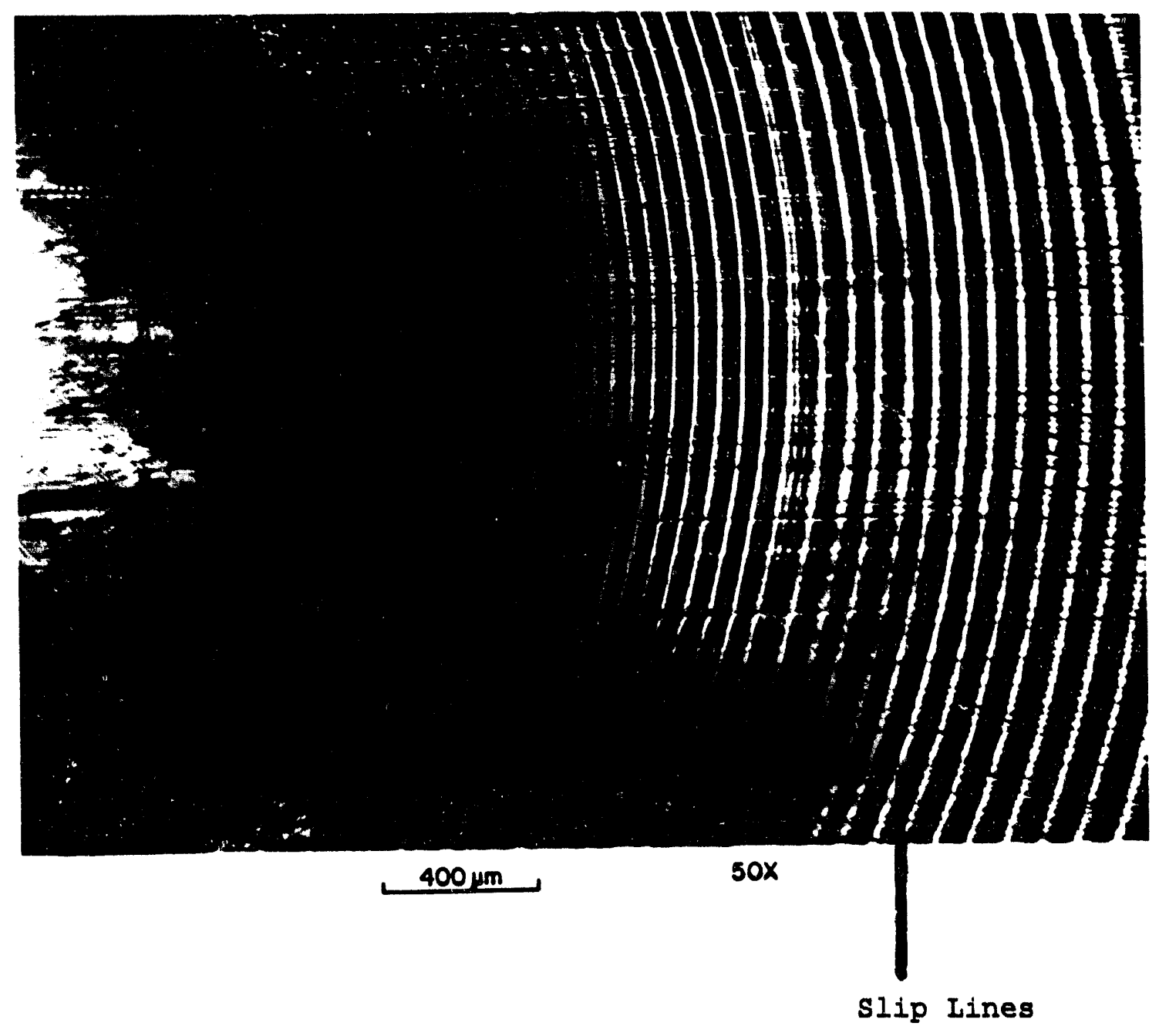

Figure 63

(001) GTA Rotational Weld in the As-Welded Condition

Dendrites in the [100] Directions, Slip Lines, and Weld Ripples (Area "A" of Figure 62) 


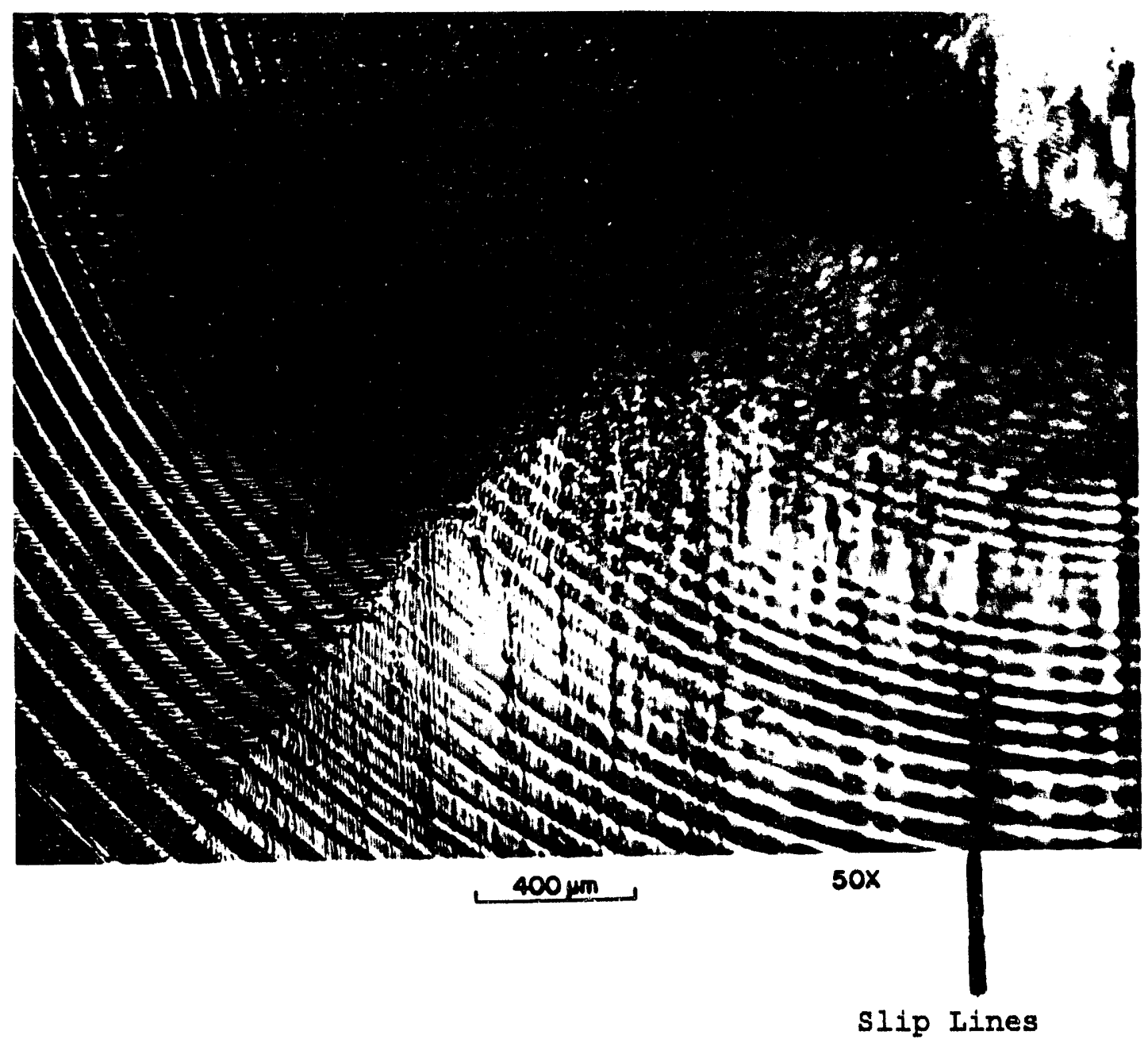

Figure 64

(001) GTA Rotational Weld in the As-Welded Condition Dendrites in the [100] Directions, Slip Lines, and Weld Ripples (Area "B" of Figure 62) 


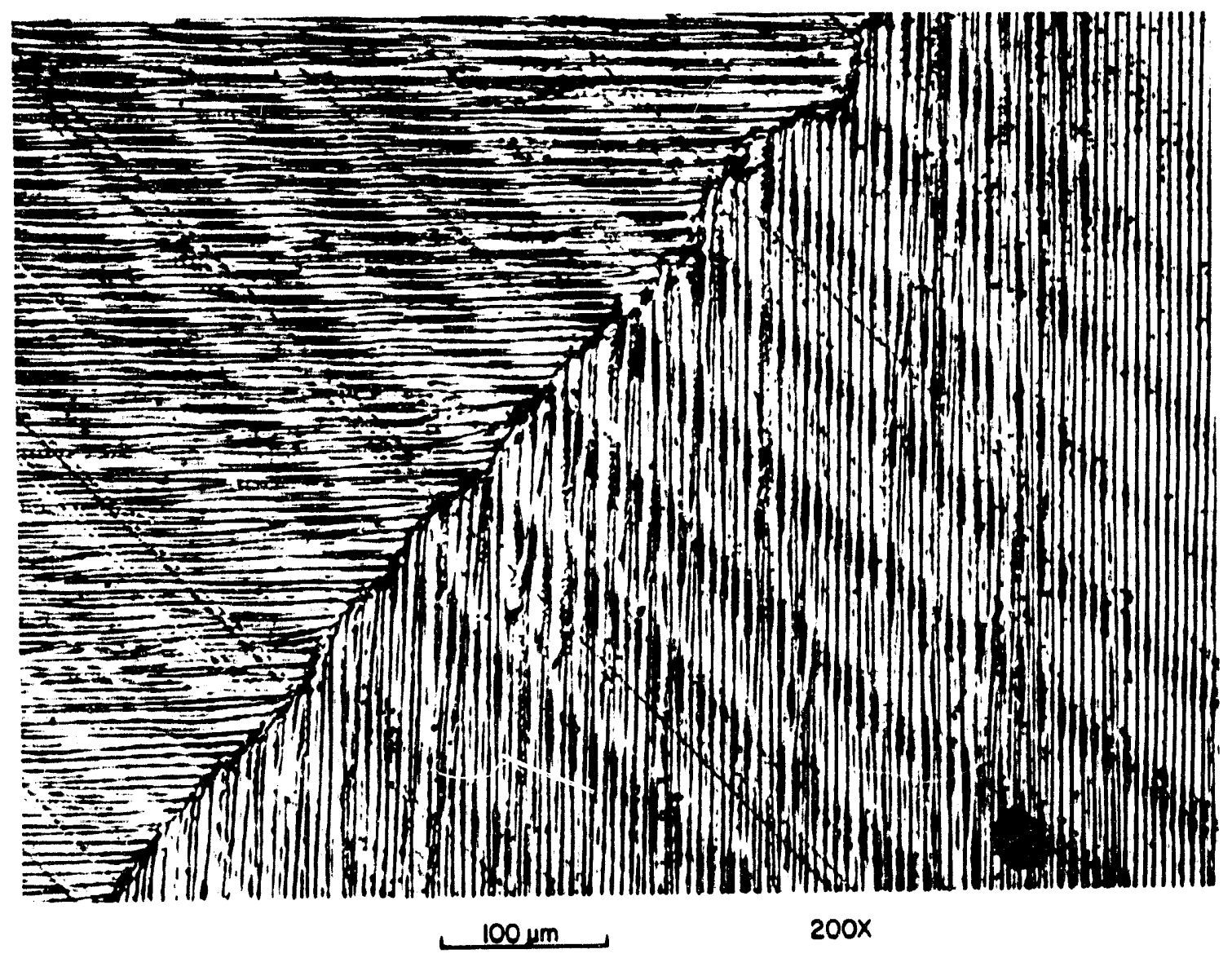

Figure 65

(001) GTA Rotational Weld

Dendrites Along the <100> Directions in the As-Welded Condition

(Area "C" of Figure 62) 


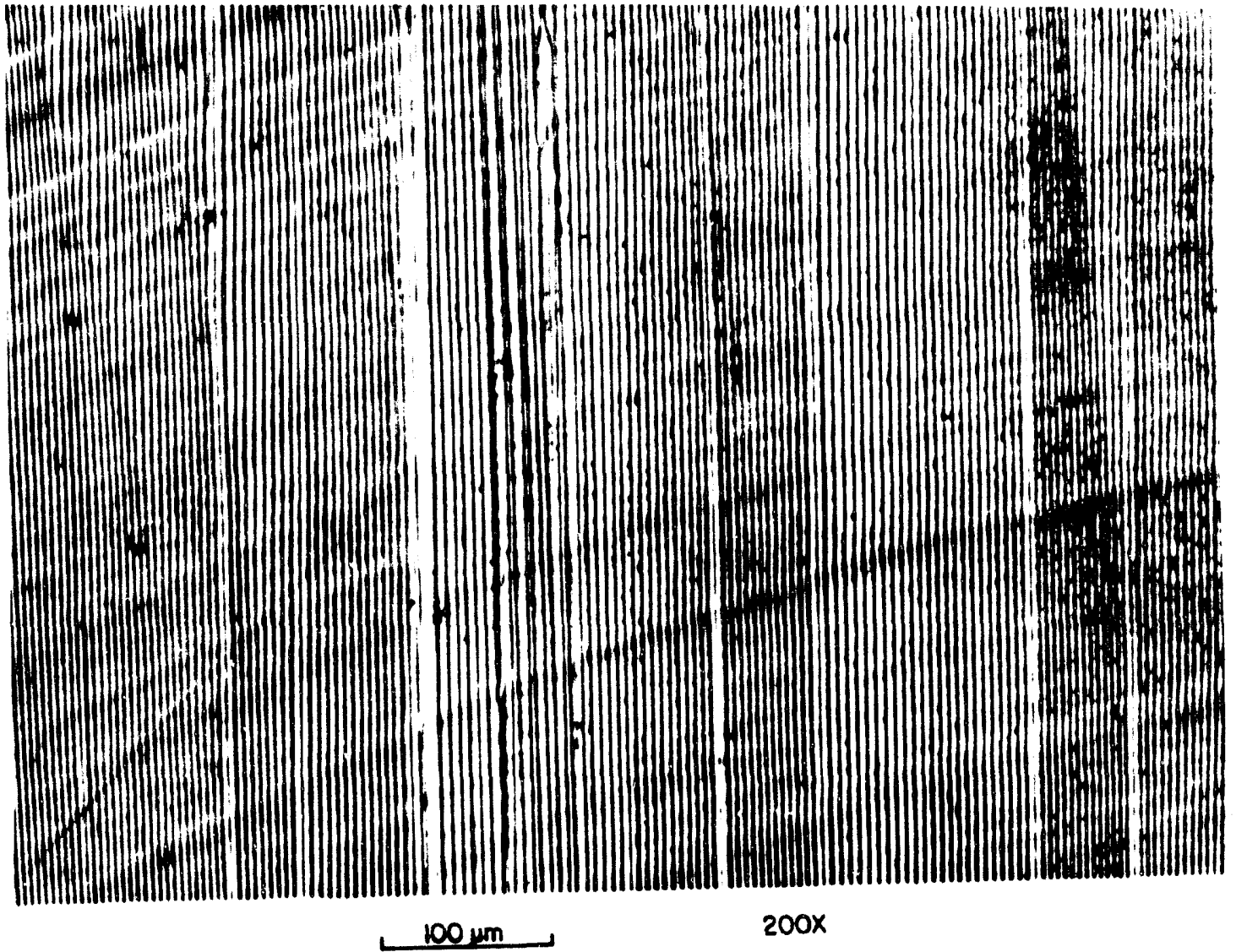

Figure 66

(001) GTA Rotational Weld

Dendrites Along the [010] Directions in the As-Welded Condition (Area "D" of Figure 62) 


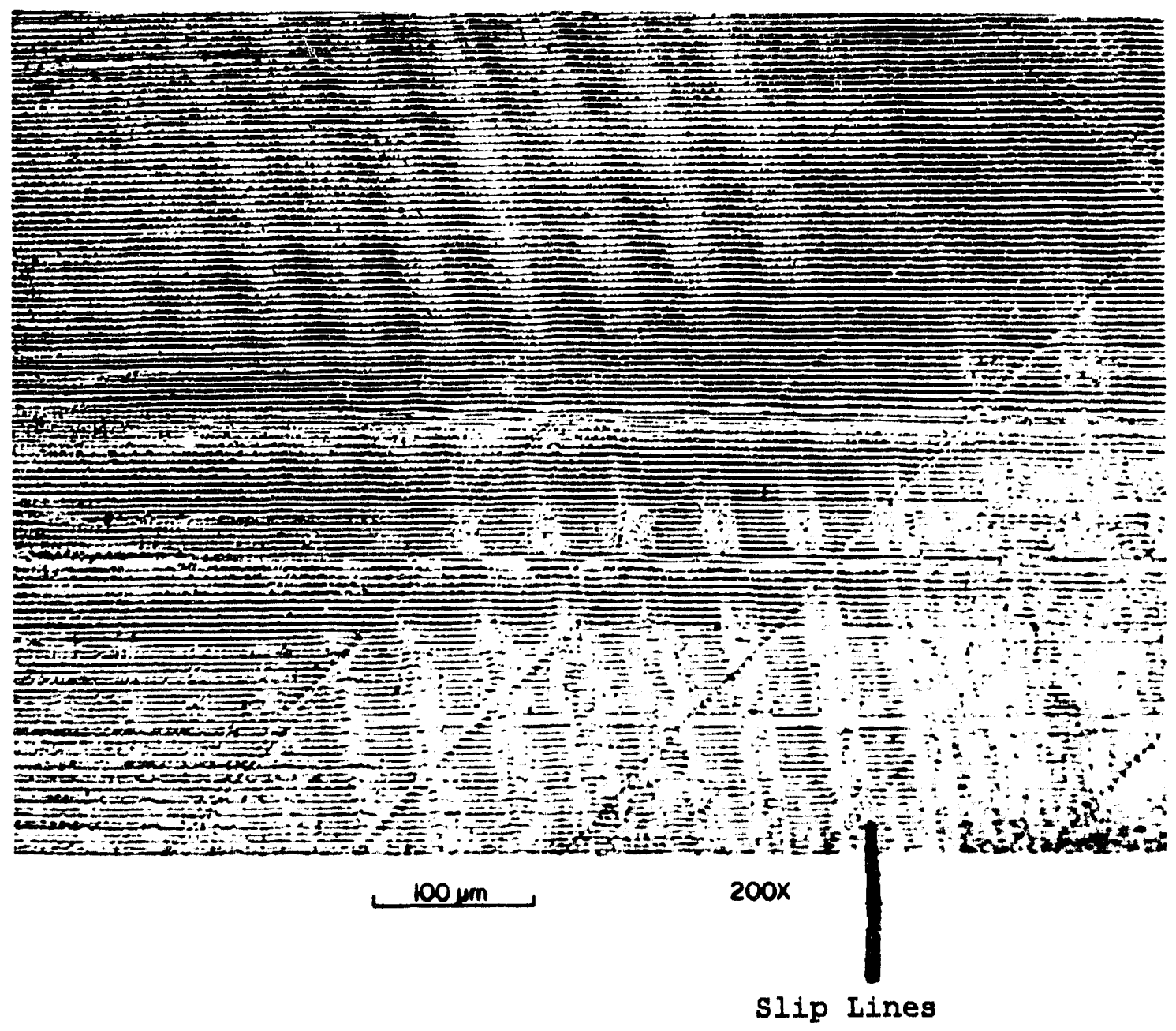

Figure 67

(001) GTA Rotational Weld in the As-Welded Condition Dendrites Along the [100] Direction and Slip Iines Visible (Area "E" of Figure 62) 
on the surface. Area "F" of Figure 62 is represented in Figure 68, illustrating a higher magnification of the dendrites and the slip lines visible in the as-welded condition. This was unique because the dendritic growth was observed in the as-welded condition and showed the clarity which can be obtained from single-crystal analysis.

\section{(011) Crystallographic orientation}

The three-dimensional reconstructions from the three transverse views, [100], [110], and [111] directions, and the top-surface "in-depth" view for GTA rotational welds are presented with the appropriate photomacrographs in Figures 69, 70, 71, and 72. As stated previously, the solidification patterns were similar to the GTA stationary welds, and thus, the resulting microstructures are similar. For details, refer to the discussion of the (011) plane for the stationary GTA welds. Figure 73 represents a three-dimensional reconstruction with areas " $A$ ", "B", and " $C$ " noted in which further investigation was performed. Area " $A$ " of Figure 73 is microstructurally represented in Figure 74 and it illustrates the transverse view along the [110] direction demonstrating the uniformity with which the interior set of $\langle 100\rangle$ dendrites met along the centerline. The comparative growth rates of the two sides were approximately equal, as in the stationary case. The uniformity evident in area 


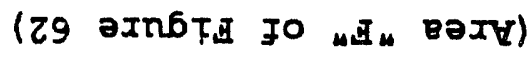

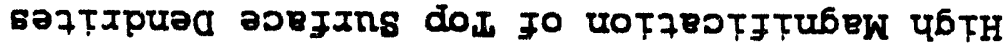

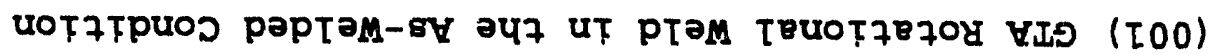

89 อxก6Ta

xoos

Wh OS

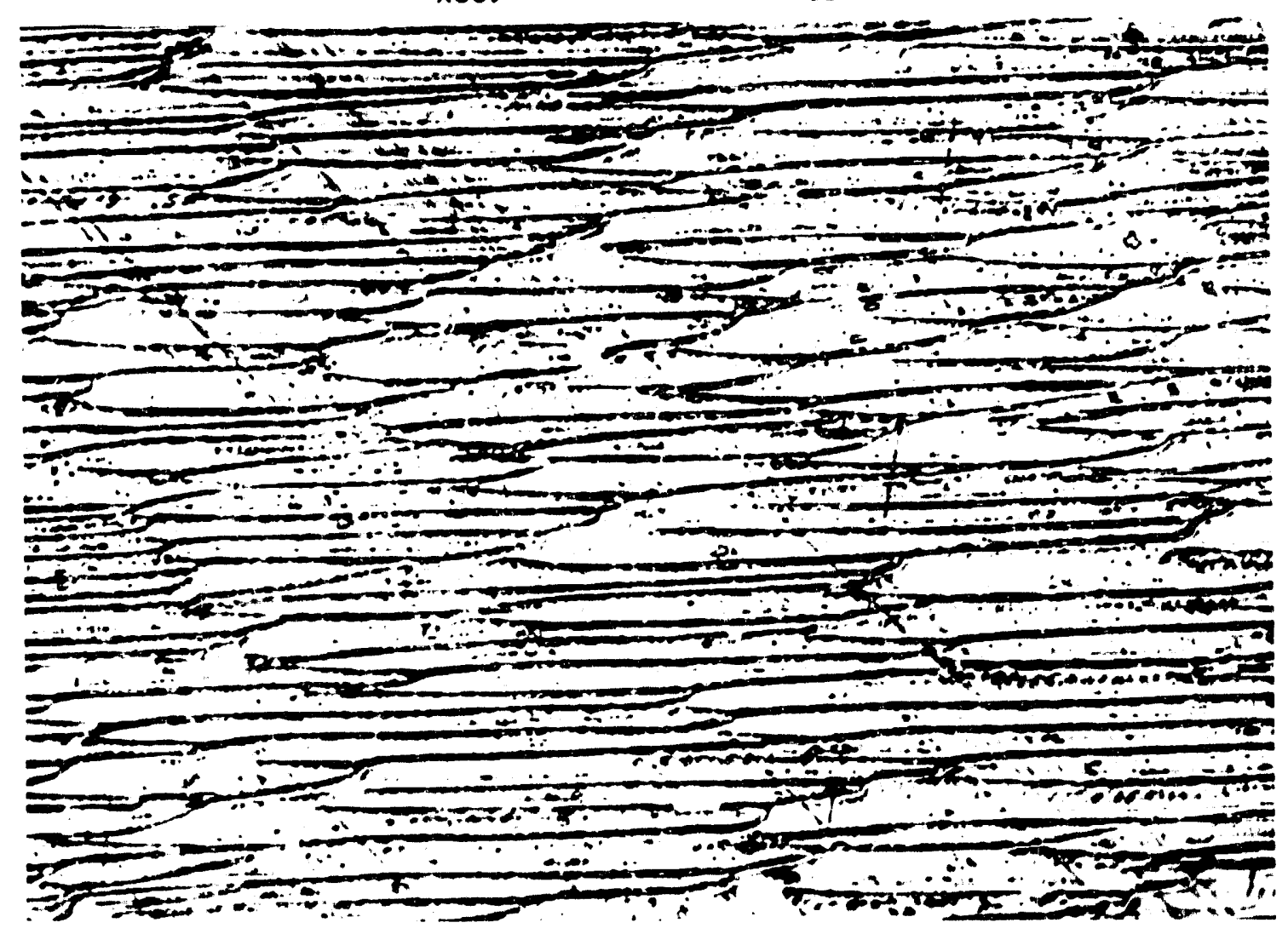

1. 


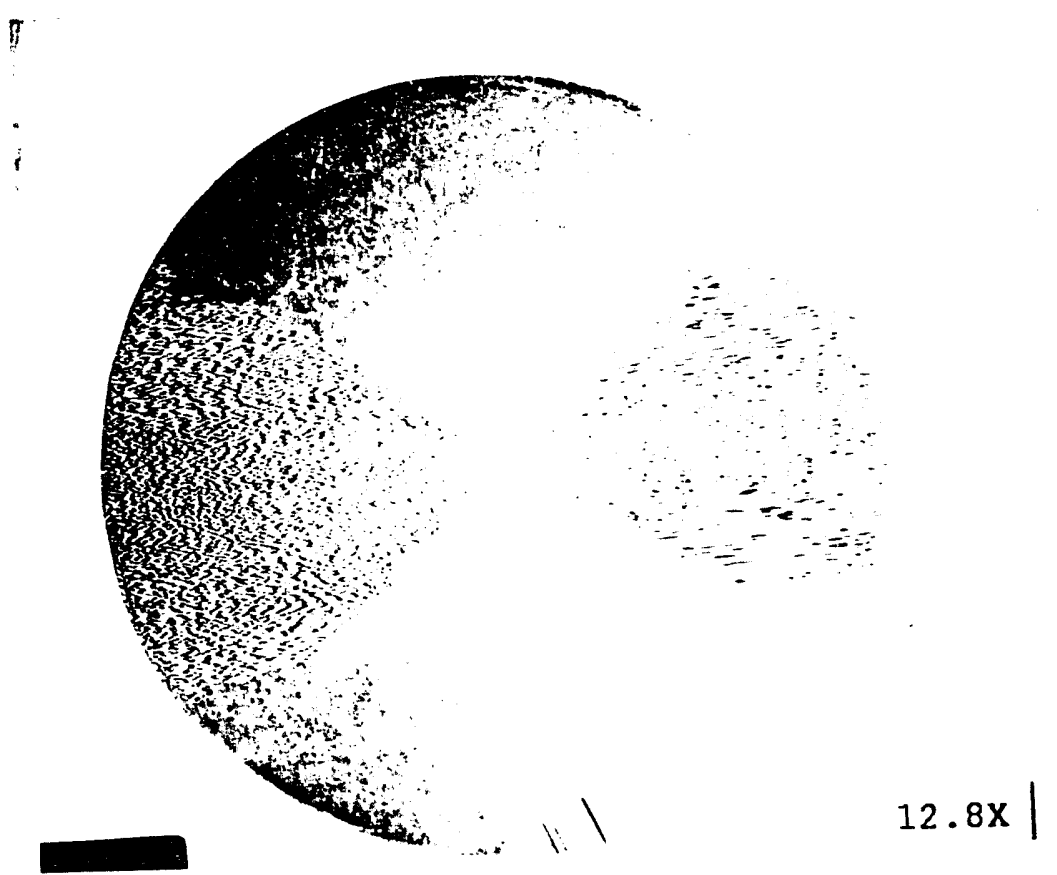

(b)

(011) Surface View Rotational GTA Weld Top of Weld

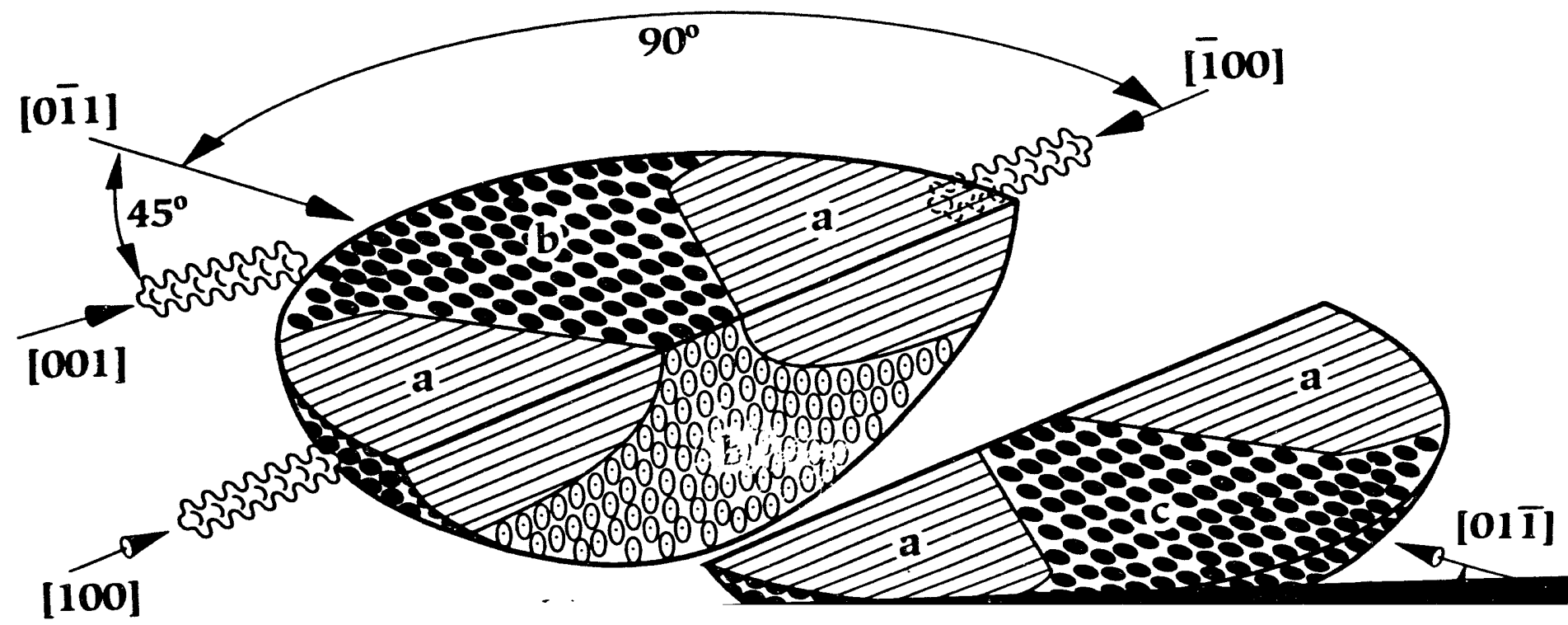


(011) Surface

Gas Tungsten Arc Weld

Y-18.

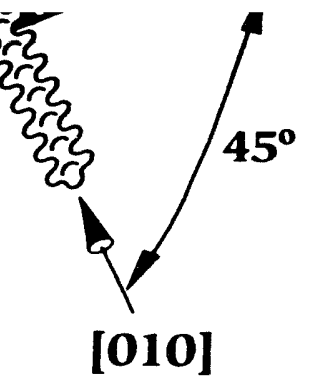

(a)

Three-dimensional Reconstruction of the Resultant Microstructure

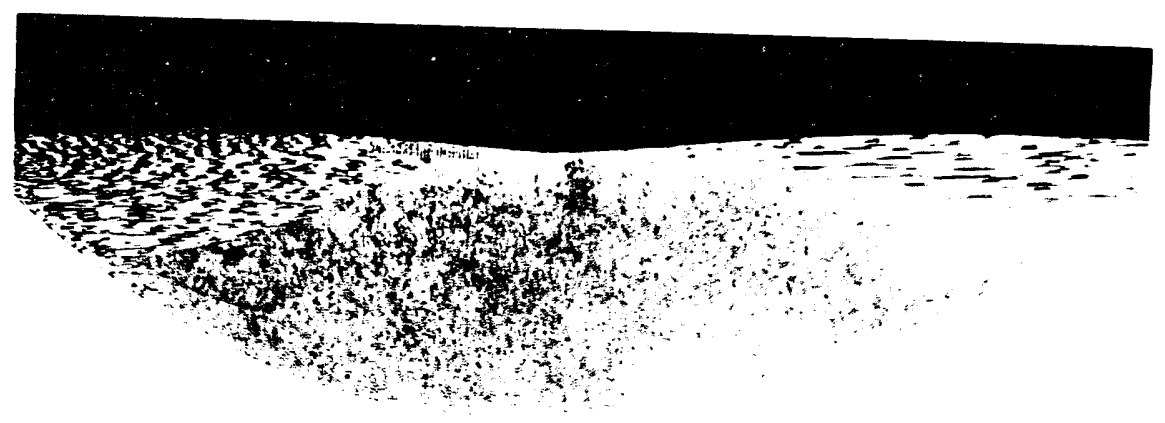

$16 \mathrm{X}$

(c)

Transverse View GTA Rotational Weld [100] Direction

Figure 69

Three-dimensional Reconstruction (011) Plane [100] Direction

Rotational GTA Weld with Accompanying Photomacrographs 


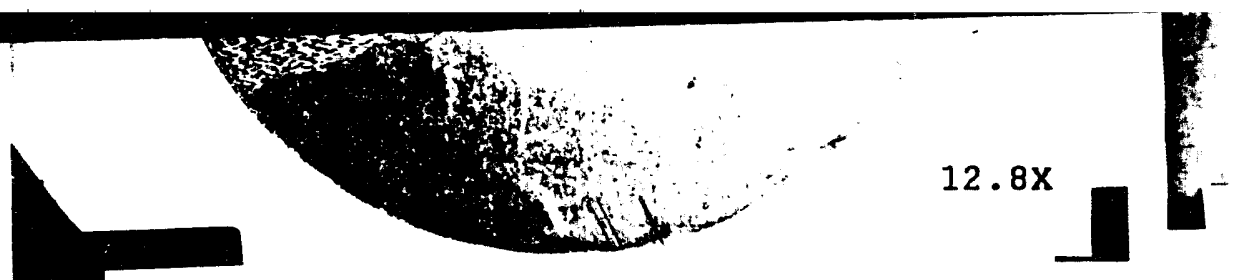

(b)

(011) Rotational GTA Weld Top Surface

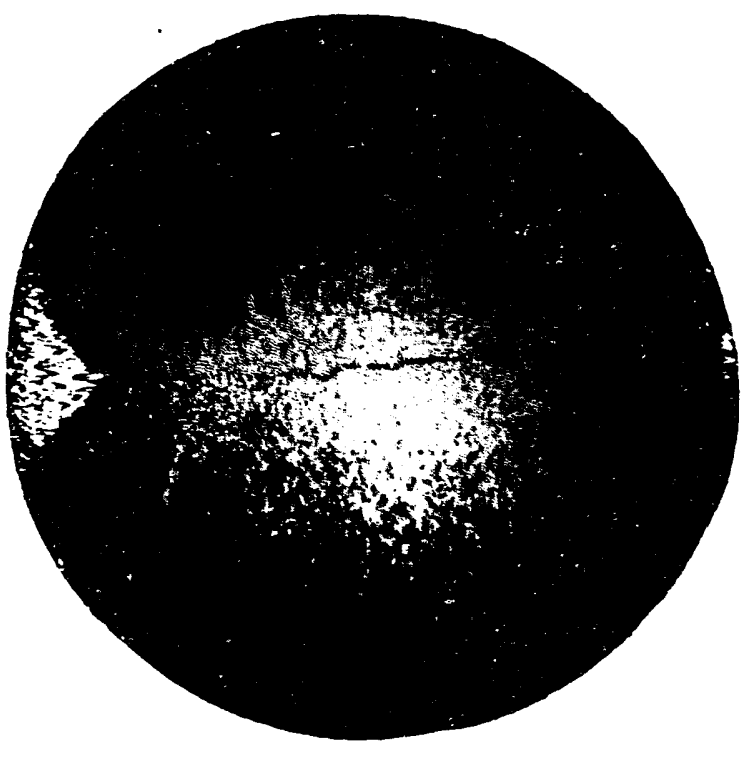

(c)

(011) Rotational GTA Weld Increased Depth into Top Surface
$12.8 \mathrm{x}$

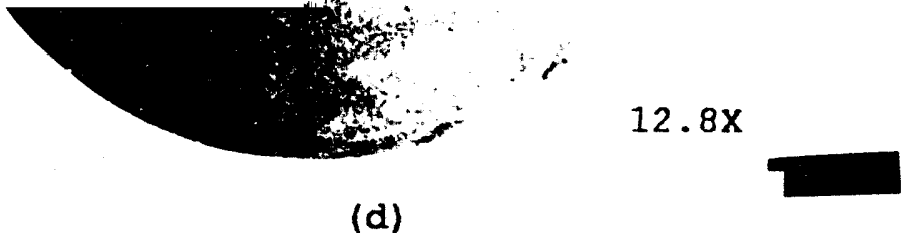

(d) Increased Depth into Top Surface

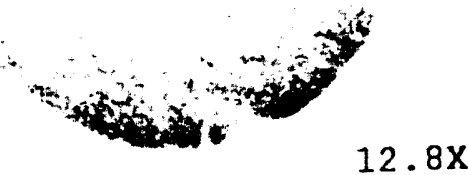

(e) 


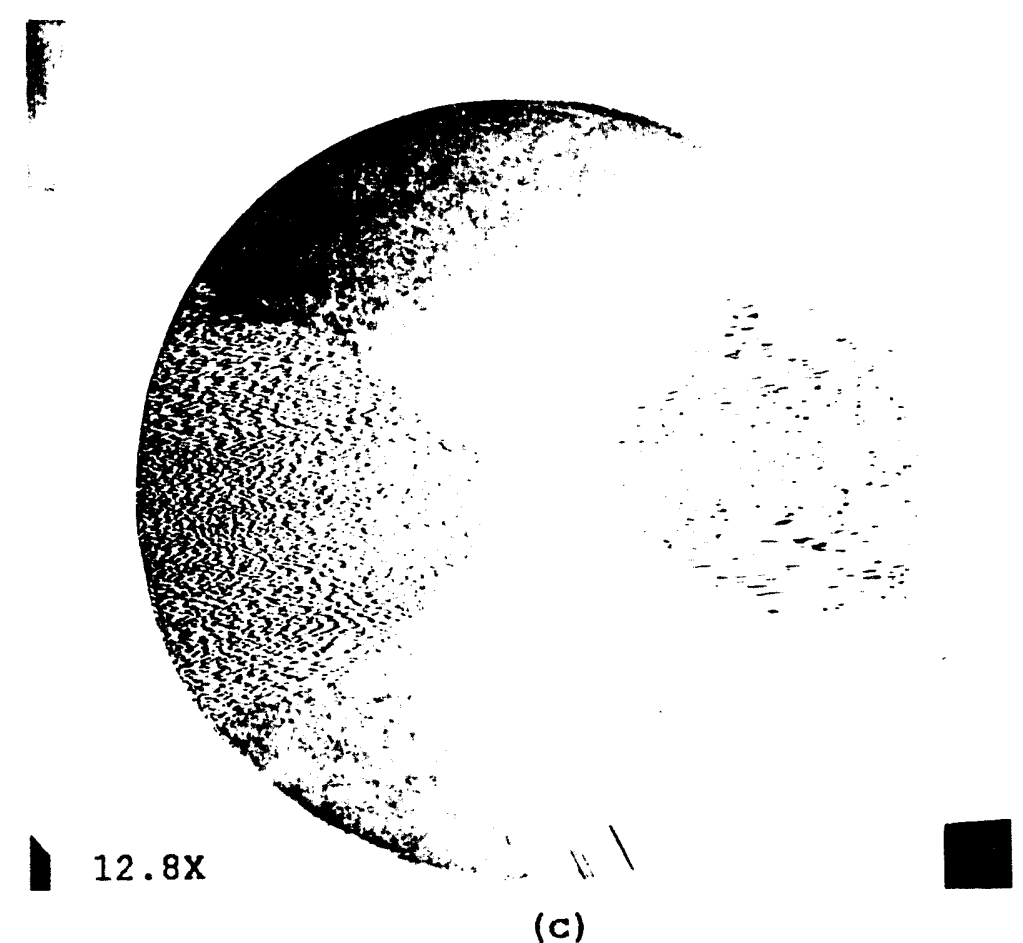

(011) Surface View Rotational GTA Weld Top Surface

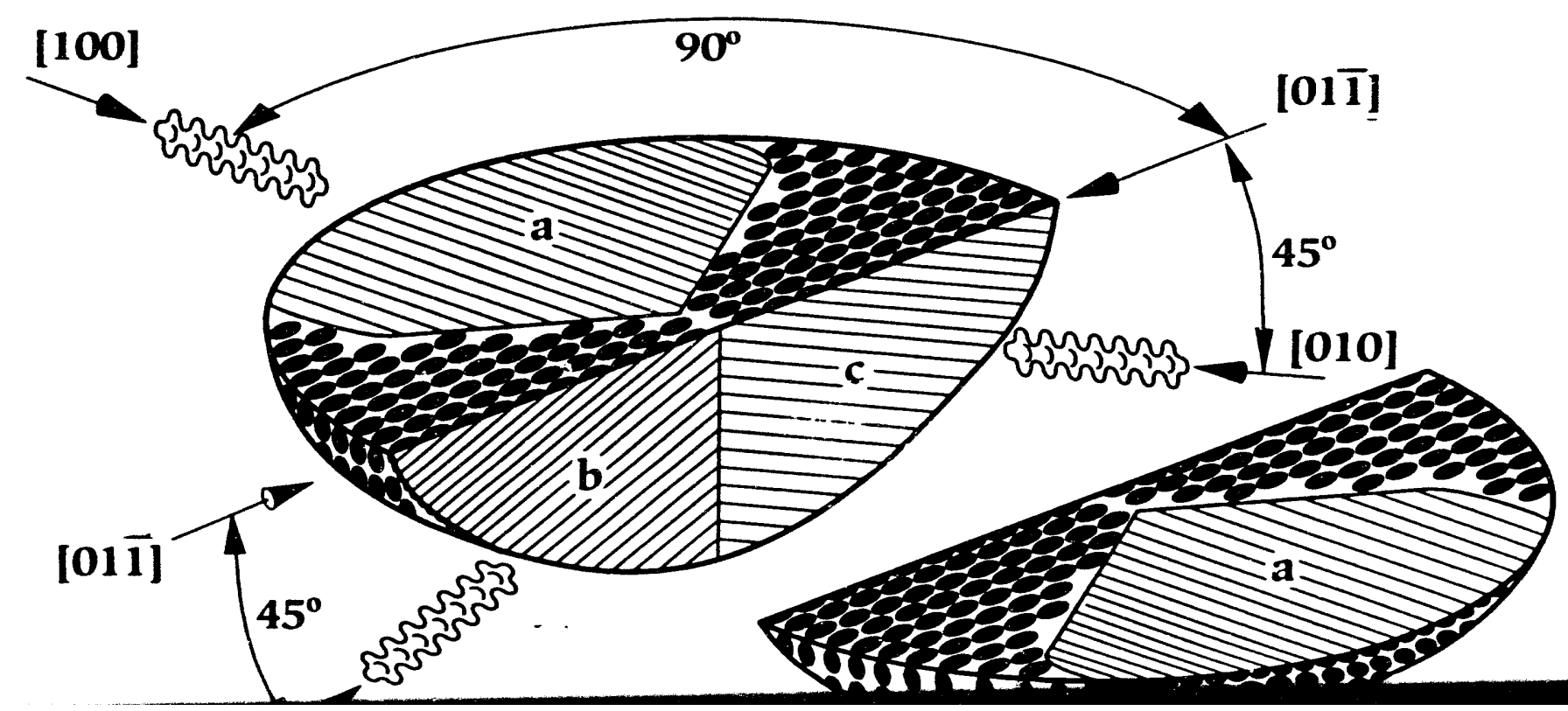


[001]

\section{(011) Surface}

\section{Gas Tungsten Arc Weld}

(a)

Three-dimensional Reconstruction of the Resultant Microstructure

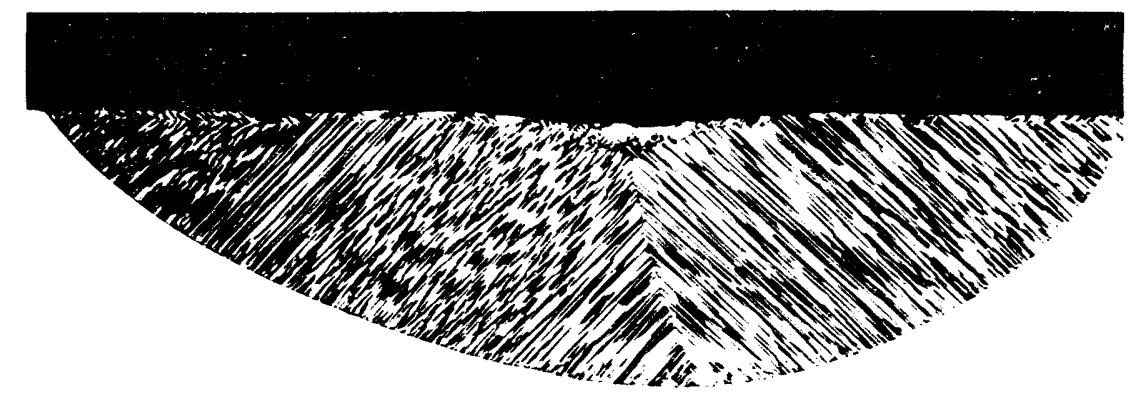

$16 \mathrm{x}$

(b)

Transverse View GTA Rotational Weld [110] Direction

\section{Figure 71}

Three-dimensional Reconstruction (011) Plane [110] Direction

Rotational GTA Weld with Accompanying Photomacrographs 


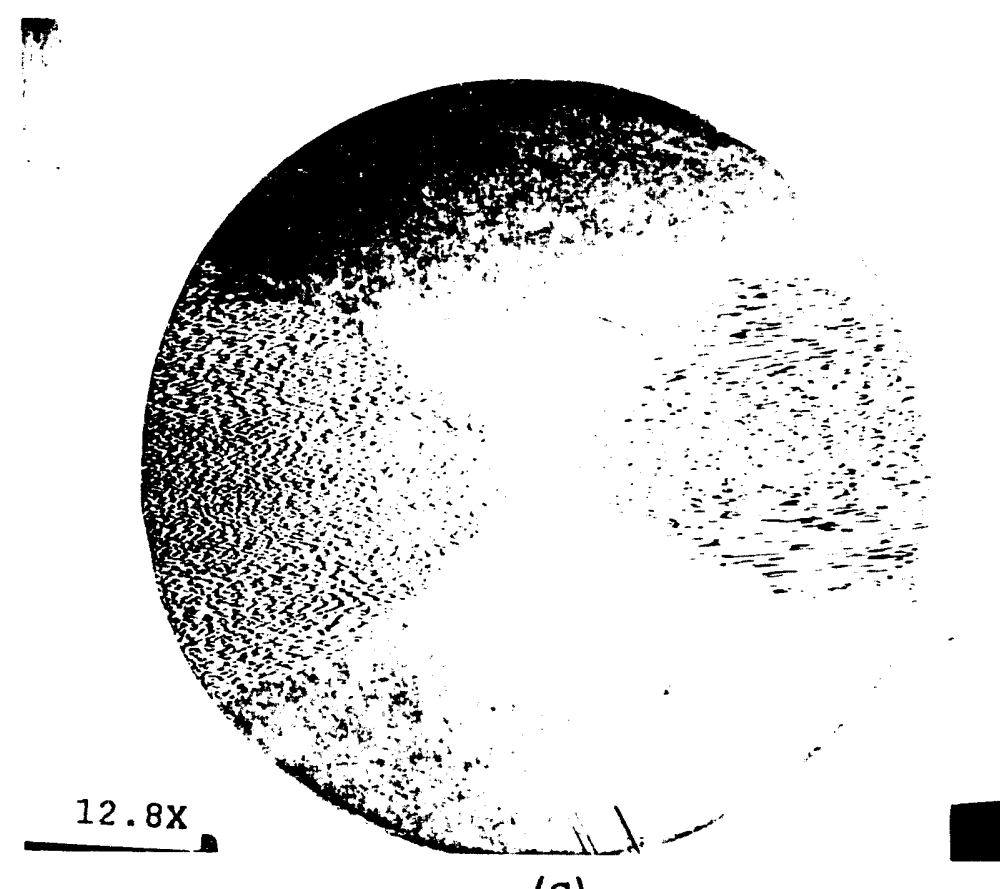

(c)

(011) Surface View Rotational GTA Weld Top Surface

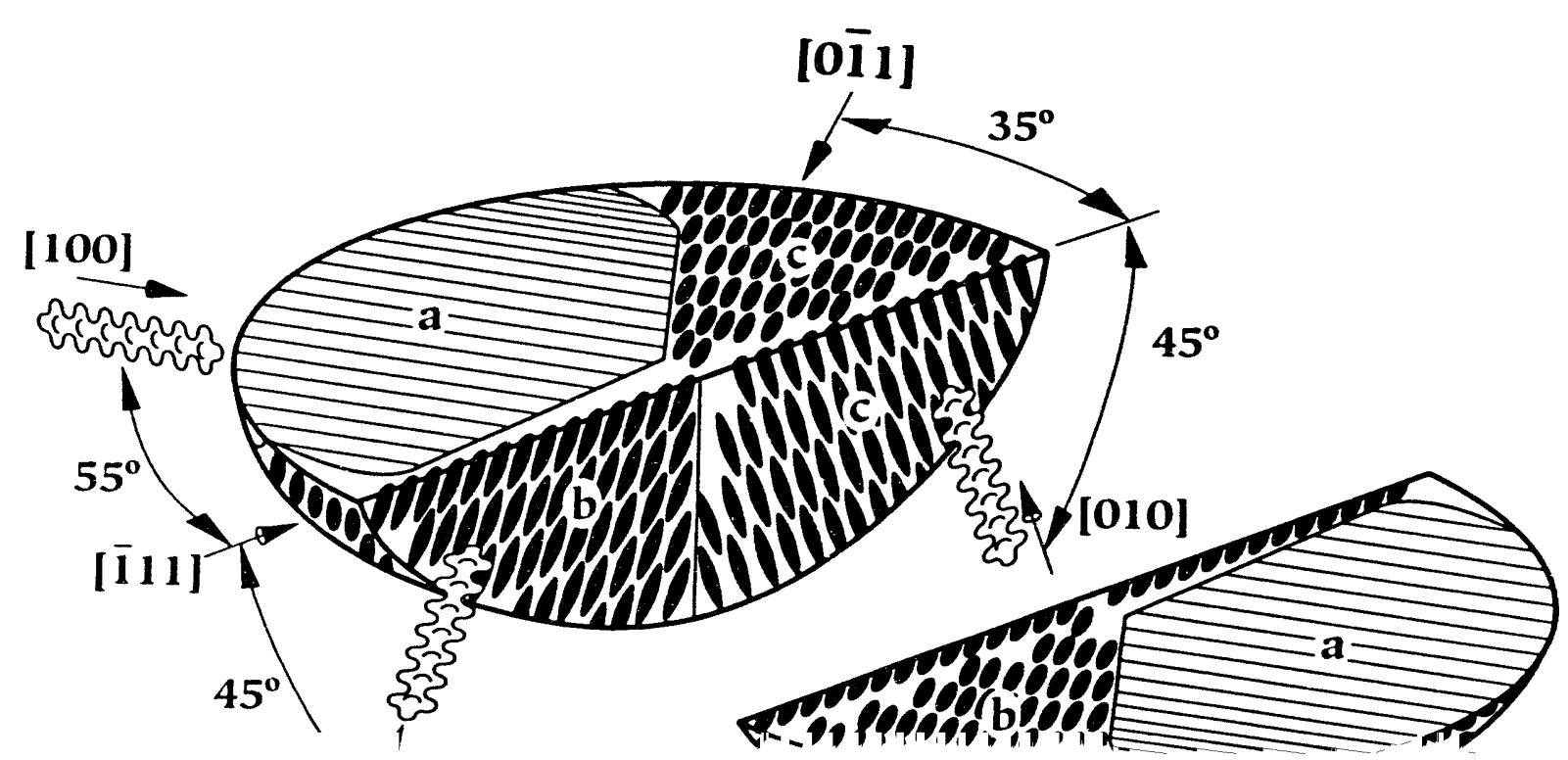




\section{(011) Surface}

Gas Tungsten Arc Weld

(a)

Three-dimensional Reconstruction of the Resultant Microstructure

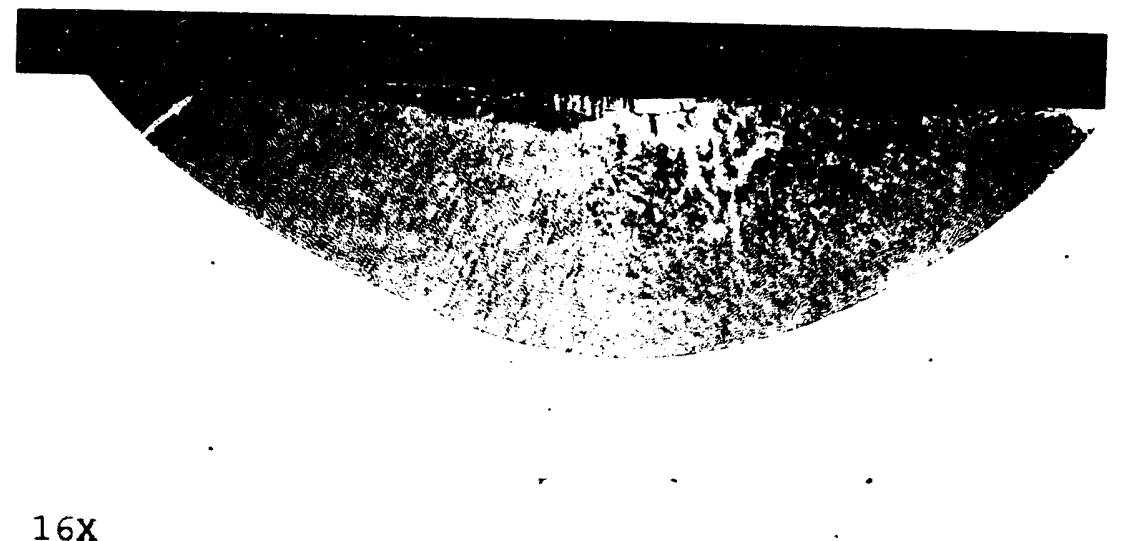

(b)

Transverse View GTA Rotational Weld [111] Direction

F1gure 72

Three-dimensional Reconstruction (011) Plane [111] Direction Rotational GTA Weld with Accompanying Photomacrographs 


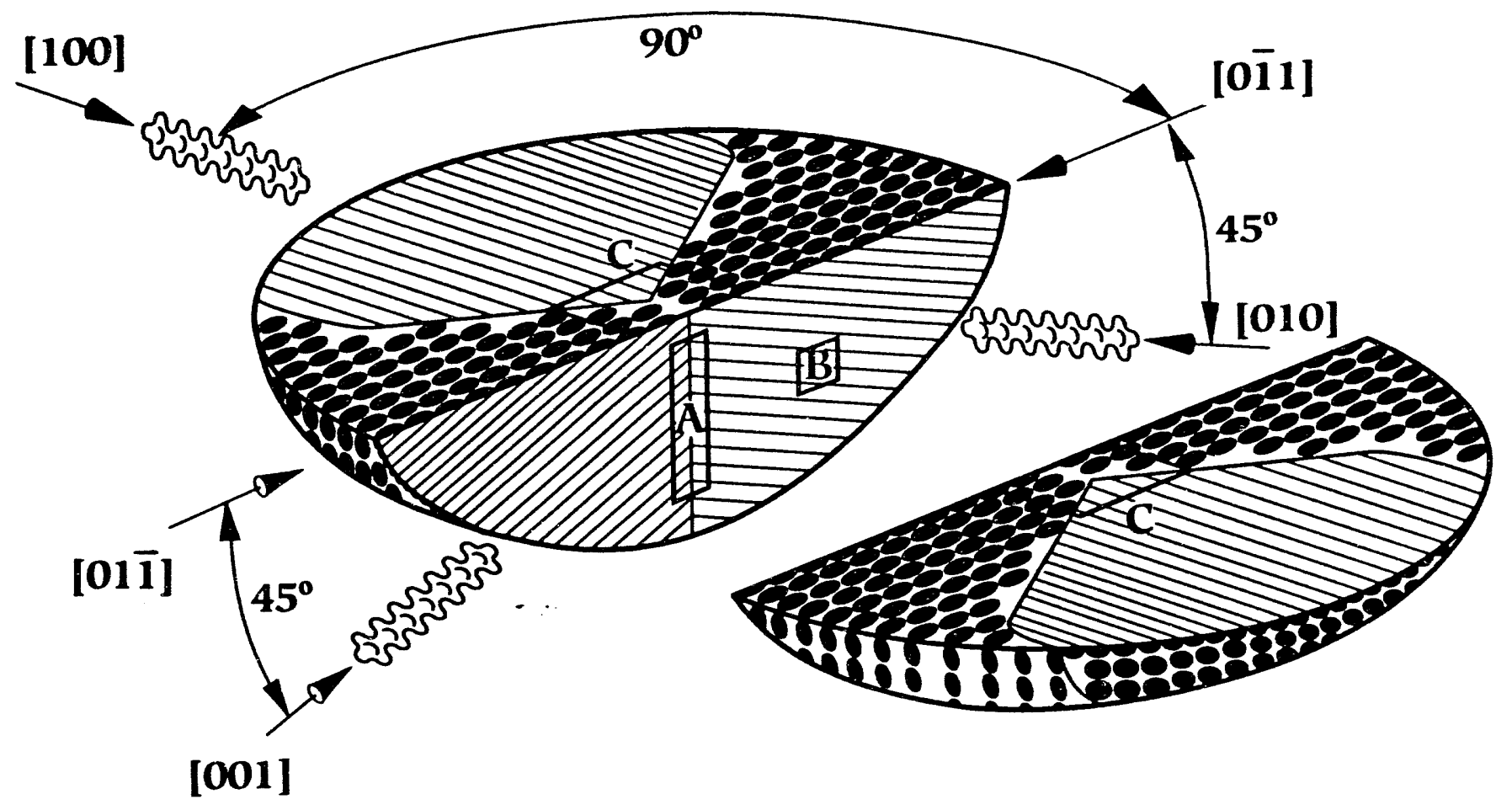

(011) Surface

Gas Tungsten Arc Weld

Figure 73

Three-dimensional Reconstruction

Identifying High Magnification Areas 

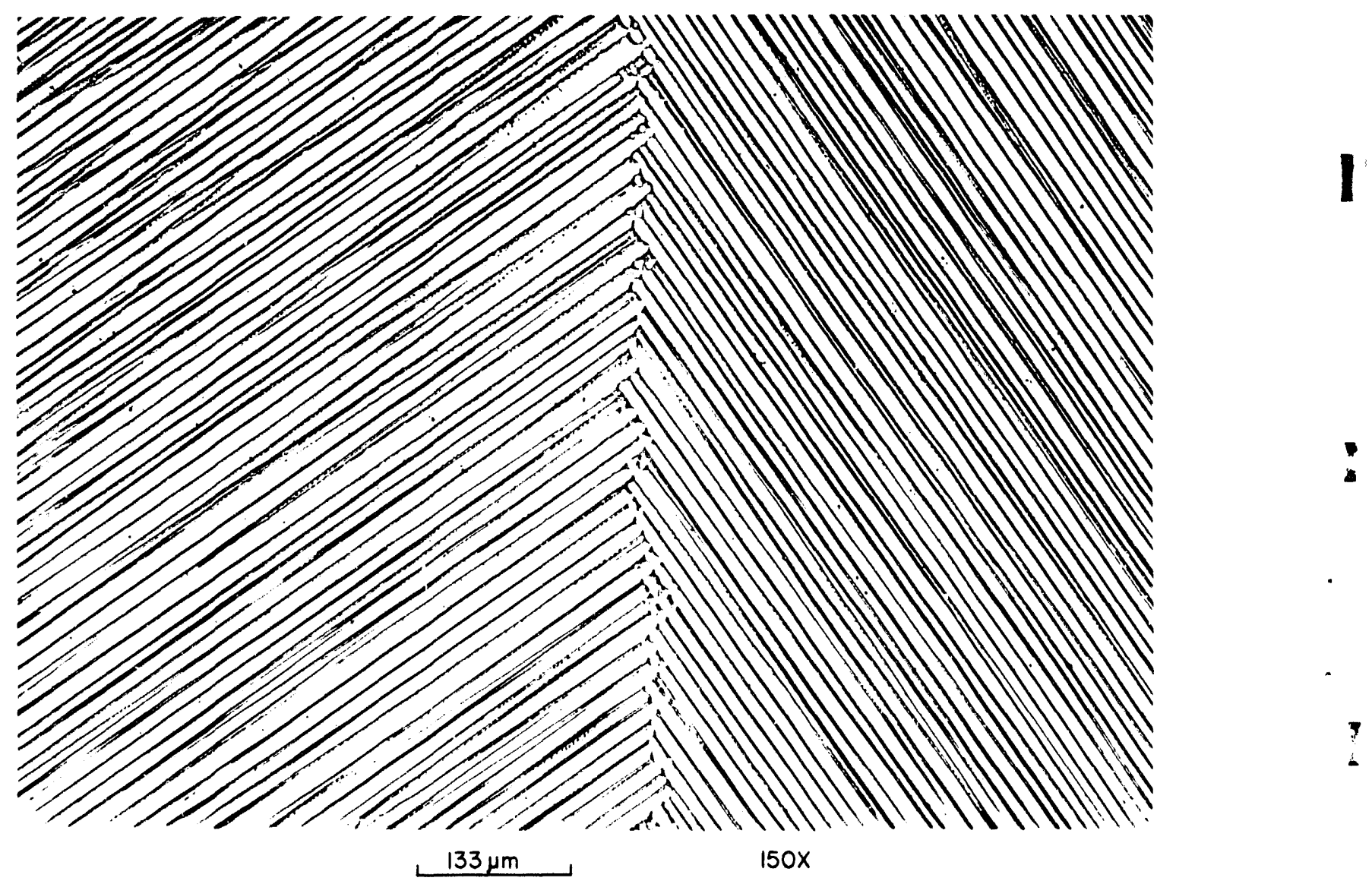

Figure 74

(011) Rotational GTA Weld symmetry in the Center of the Weld (Area "A" of Figure 73) 
"B" of Figure 73 is illustrated in Figure 75. This uniformity of the [010] dendrites is shown by the comparison of the dendriticarm spacing-i.e., the dendrites were fairly evenly spaced. Area "C" of Figure 73 is shown microstructurally in Figure 76 . It represents the symmetry of the weld pool, which is similar to the symmetry presented in the stationary-weld micrograph. All of these views can be compared to the stationary case showing the repeatability in the weld microstructure.

\section{(111) Crystallographic Orientation}

Inconsistencies in the melt-pool shape noted in the stationary GTA discussion of the (111) plane resulted in a limited availability of high quality welds. Again, the solidification patterns, and thus the resulting microstructure, were similar to the stationary-weld case. Therefore, for details on the microstructure and the solidification, refer to the stationary-welding condition for the (111) plane. Figure 77 represents the three-dimensional reconstruction for the rotational GTA weld on the (111) surface from the transverse view, the "in-depth" surface view, and the stereographic projection of this surface. The transverse view (Figure 77b) shows one of the well defined weld pool shapes which was achieved for the (111) plane. The center line is visible in this weld. 

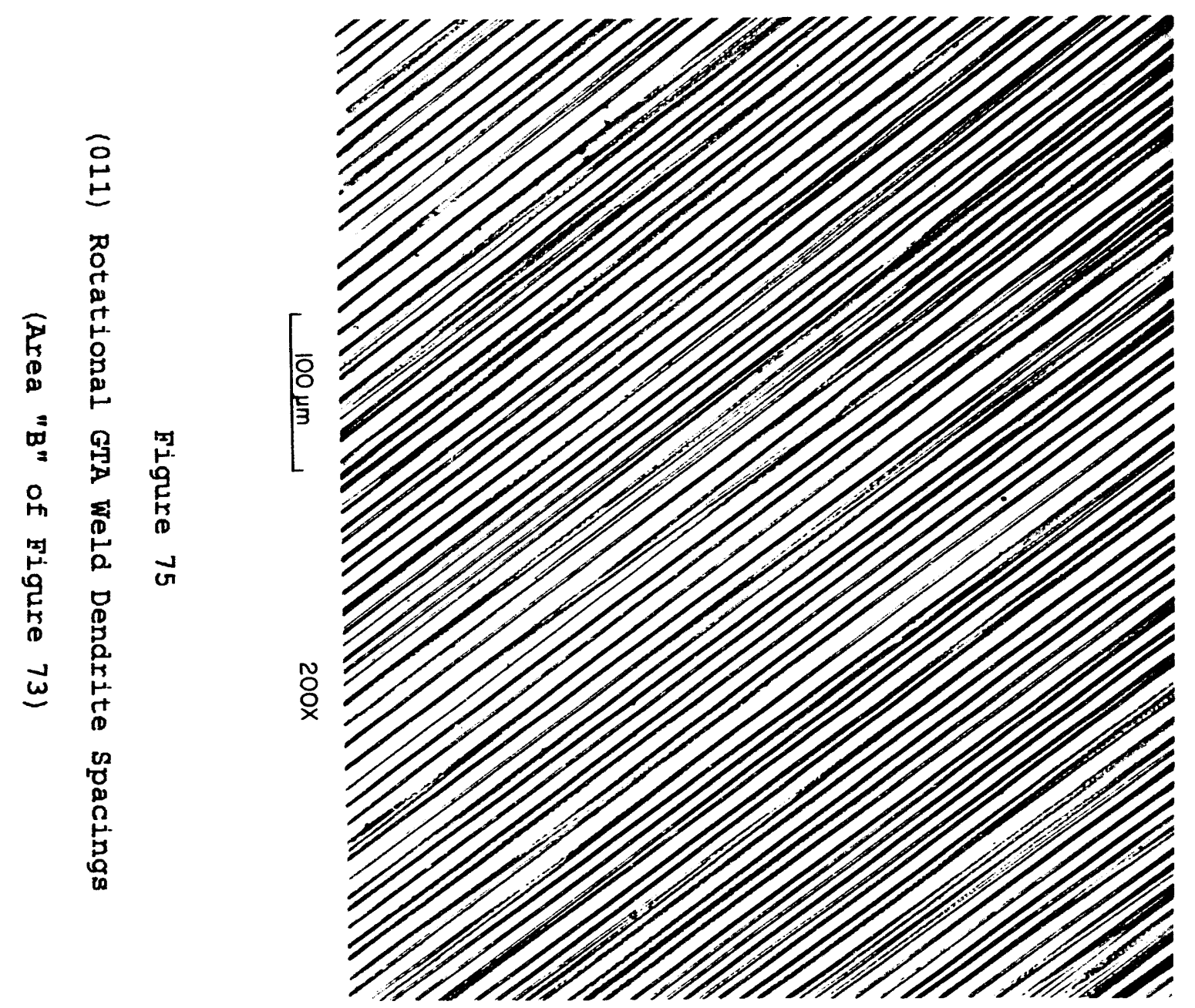

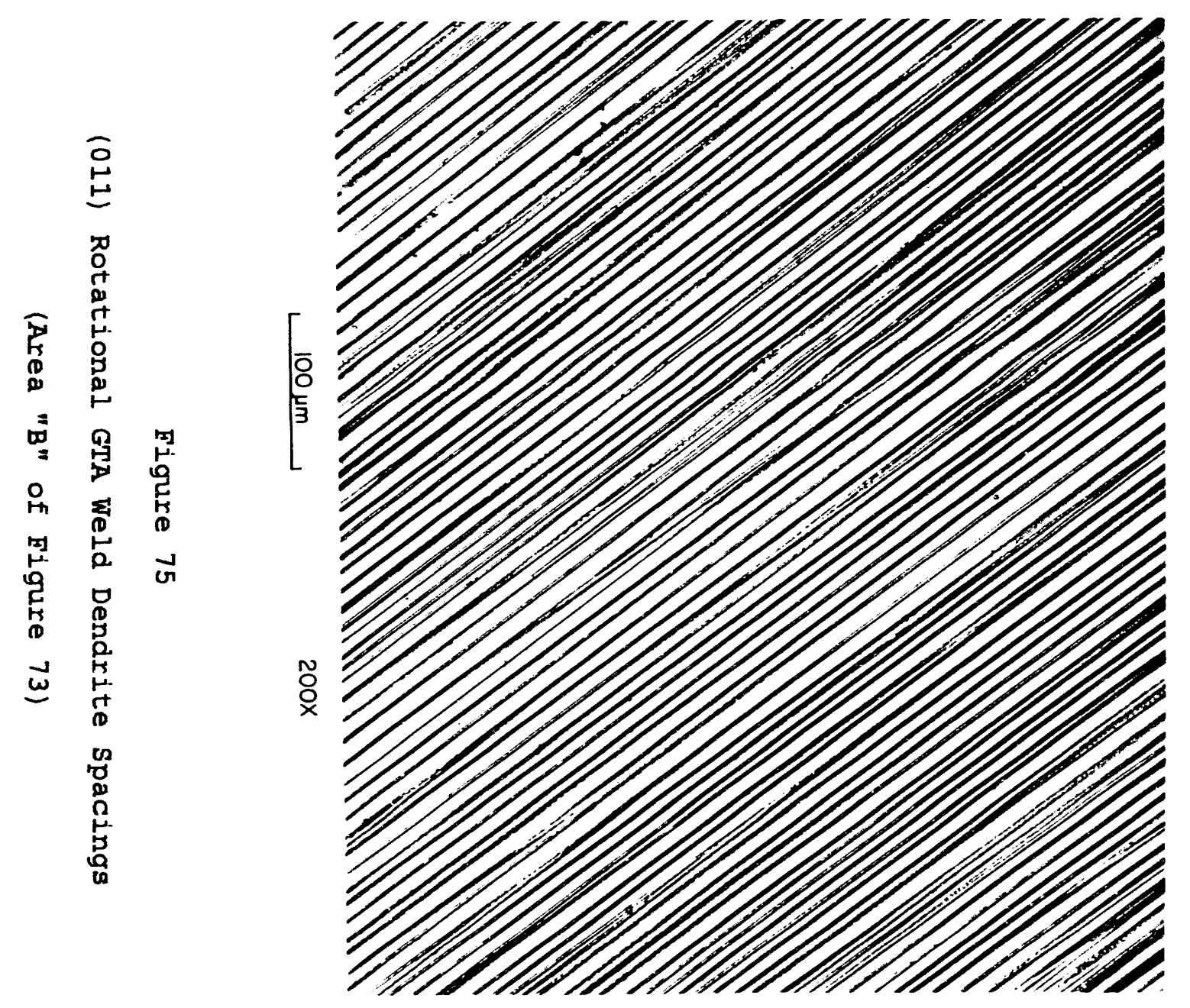


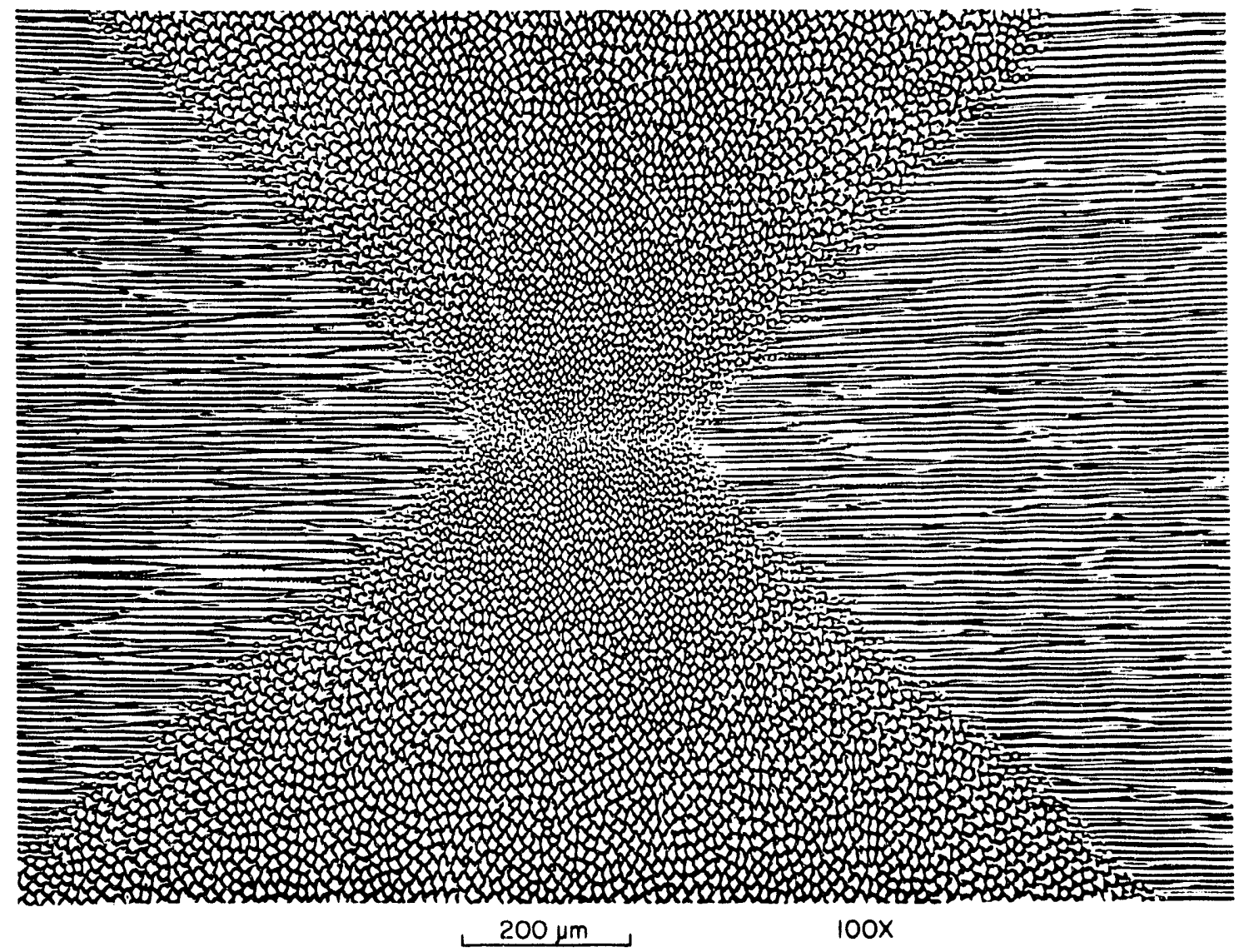

Figure 76

(011) Surface symmetry of the Center of the weld

(Area "C" of Figure 73) 


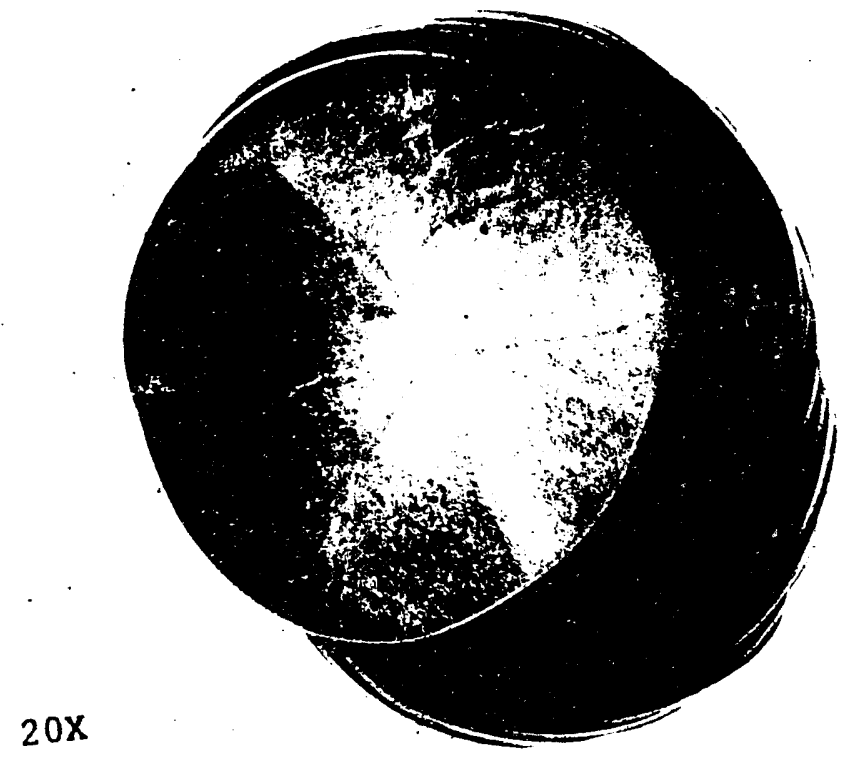

(b)

(111) Surface View Rotational GTA Weld Top Surface

(111) surface View Rotational Gid Weld Top Surface

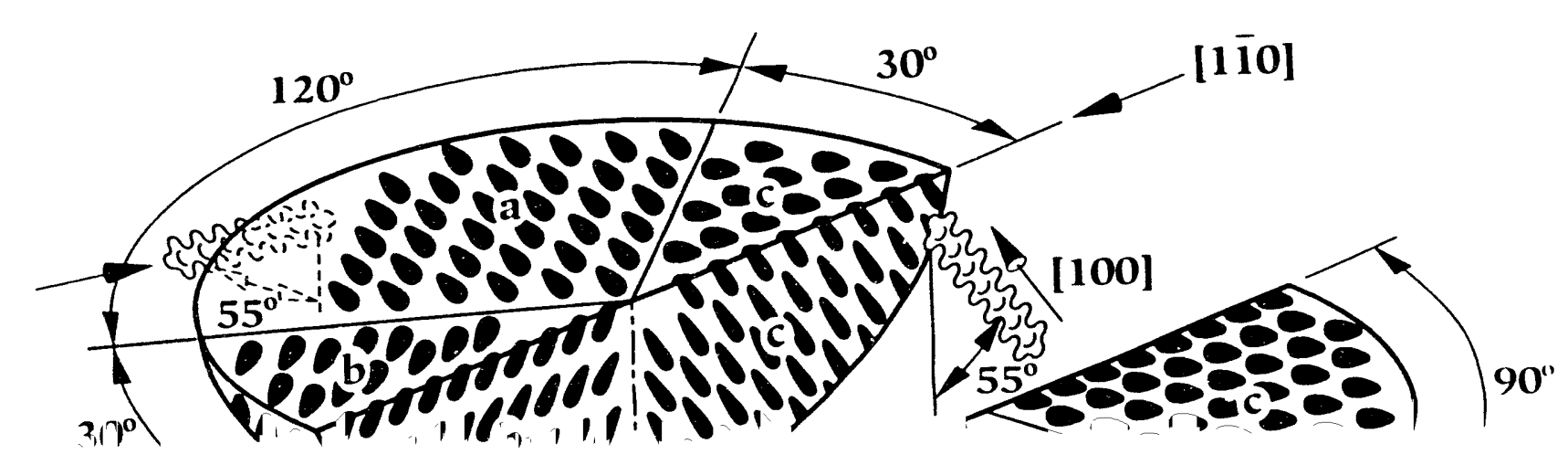




\section{[11 10]}

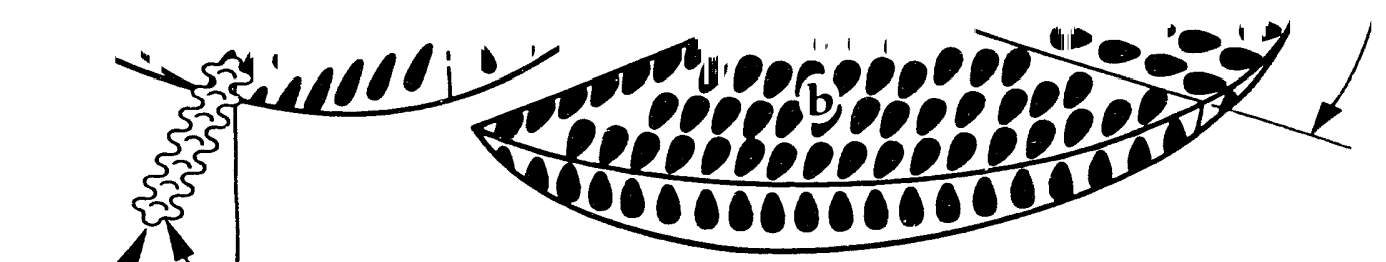

$55^{\circ}$ (111) Surface

[001]

Gas Tungsten Arc Weld

(a)

Three-Dimensional Reconstruction of the Resultant Microstructure

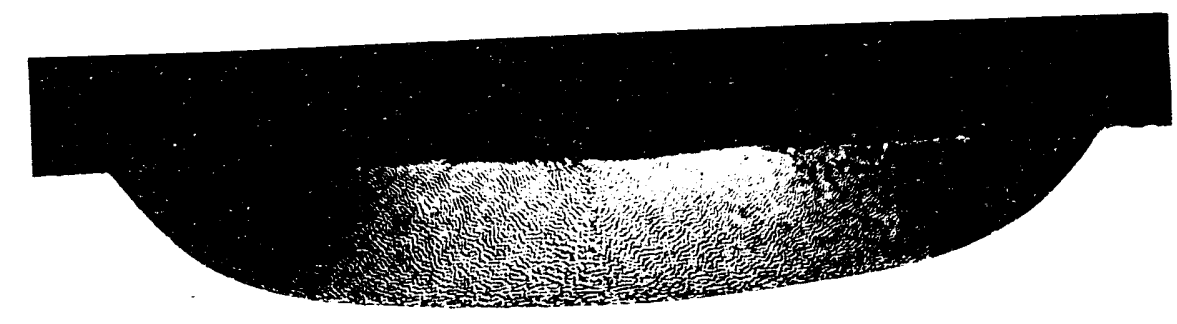

$16 \mathrm{X}$

(c)

Transverse View Rotational GTA Weld [110] Direction

Figure 77

Three-dimensional Reconstruction (111) Plane [110] Direction

Rotational GTA Weld with Accompanying Photomacrographs 
This is accompanied by the macrographs for the top surface and transverse views.

\title{
Comparison of Gas Iungsten Arc \\ Rotational to stationary Felds
}

\begin{abstract}
A comparison of the two GTA welding methods, 1.e., rotational and stetionary, revealed that the pool shape became more uniform in the transverse and surface views for the rotational welds. However, the overall solidification patterns in the two methods were similar as mentioned previously. The weld-pool shape for the stationary case indicated that uneven penetration can occur in the weld pool, resulting in a shape that is less smooth than that consistently obtained from the rotation of the sample. This could be seen in photomacrographs of both polycrystal and single crystal welds made with the mixed shielding gas (Eigure 78,79 ). In Figure 78 it is seen that the shape for the rotational weld is more of an "arc" shape throughout the entire weld pool. Whereas in the stationary weld, the shape is not as smooth in contour over the entire weld on a consistent basis. This effect is better illustrated in the single-crystal welds in Figure 79. The uneven shape of the weld pool is clearly seen in the stationary weld and can be directly compared with the smoother shape of the rotational weld.
\end{abstract}



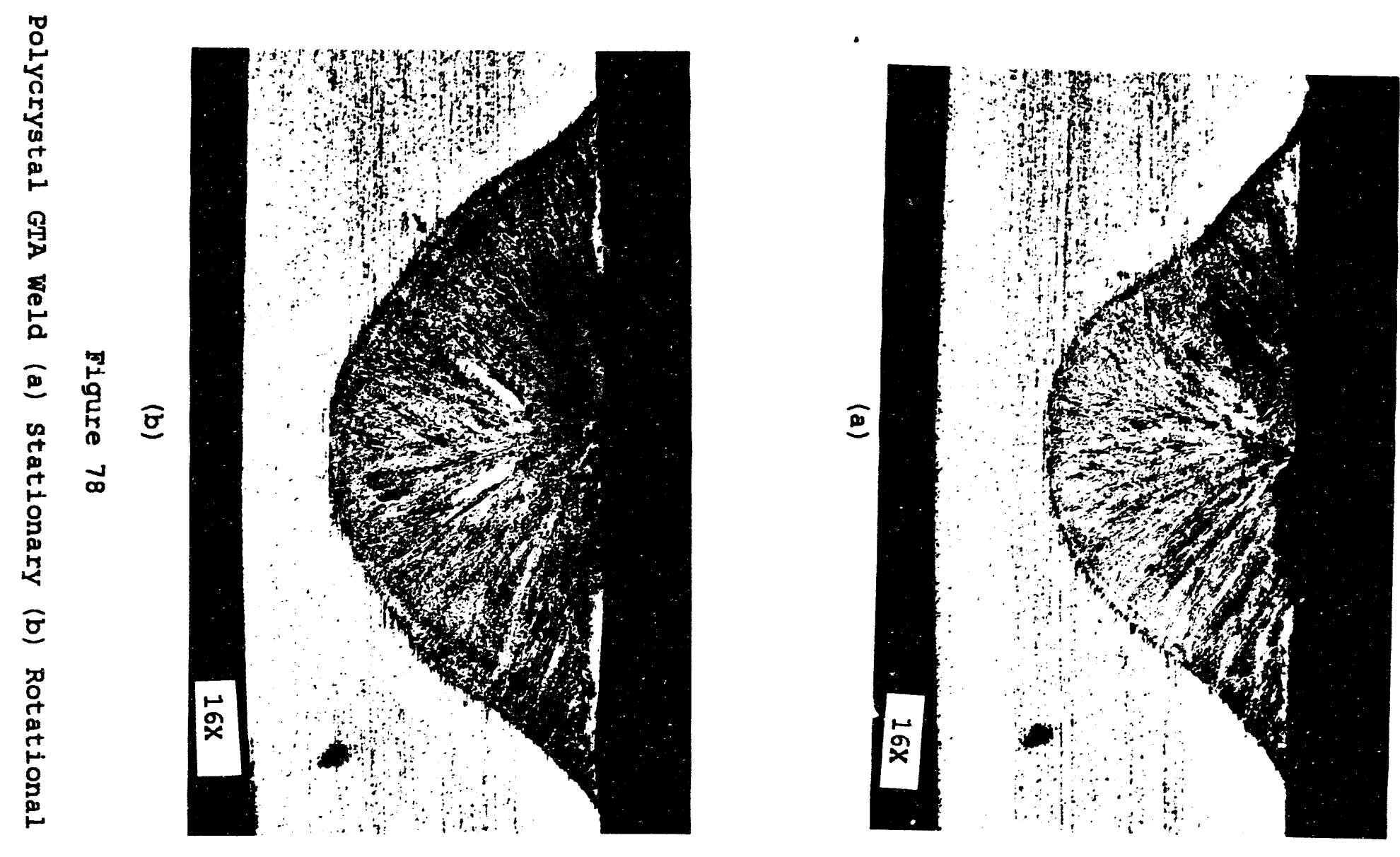


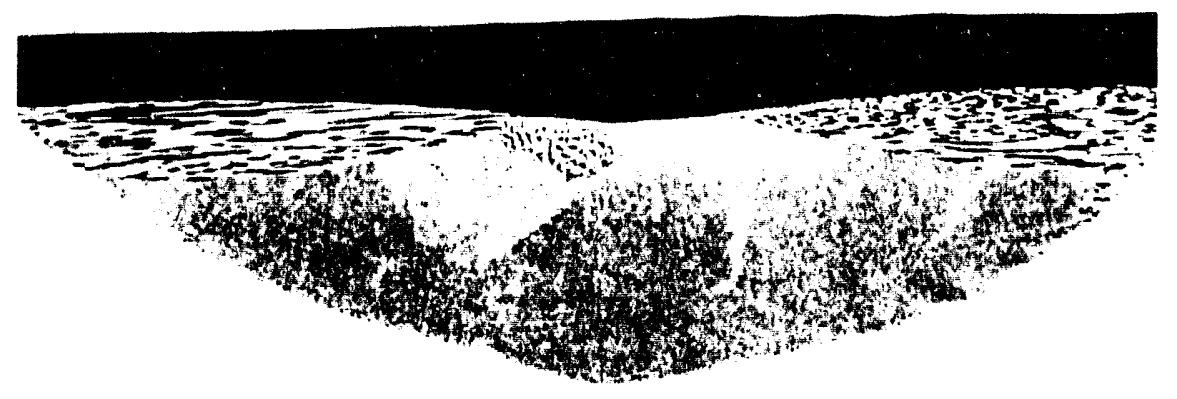

$20 x$

(a)
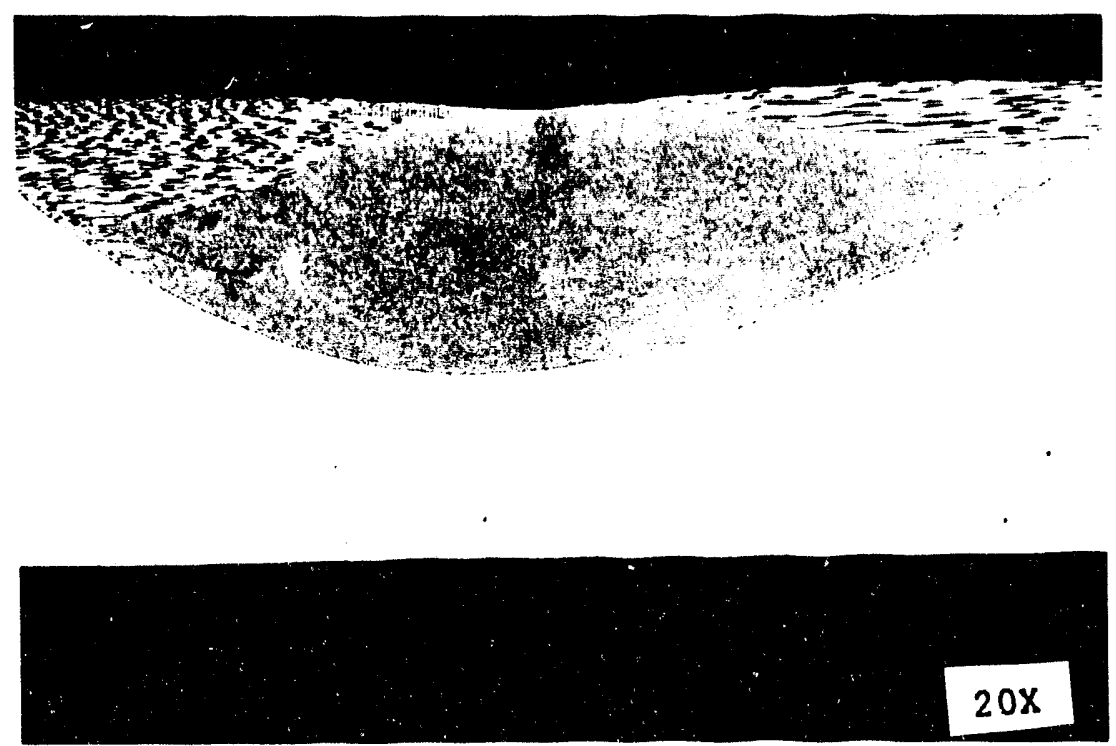

(b)

Figure 79

(011) Plane [100] Transverse View GTA Weld

(a) Stationary (b) Rotational 
The weld microstructure seemed to be consistently more symmetric in the stationary method as compared to the rotational GTA. A reason for this effect might be the formation of a double melt pool formed by the arc movement during the melting procedure (Figure 80 ). This is visible on the right-hand side of the photomacrograph showing two distinct weld pool developments. The double melt pool caused an uneven solidification pattern to develop which, in turn, influenced the symmetry of the resulting microstructure.

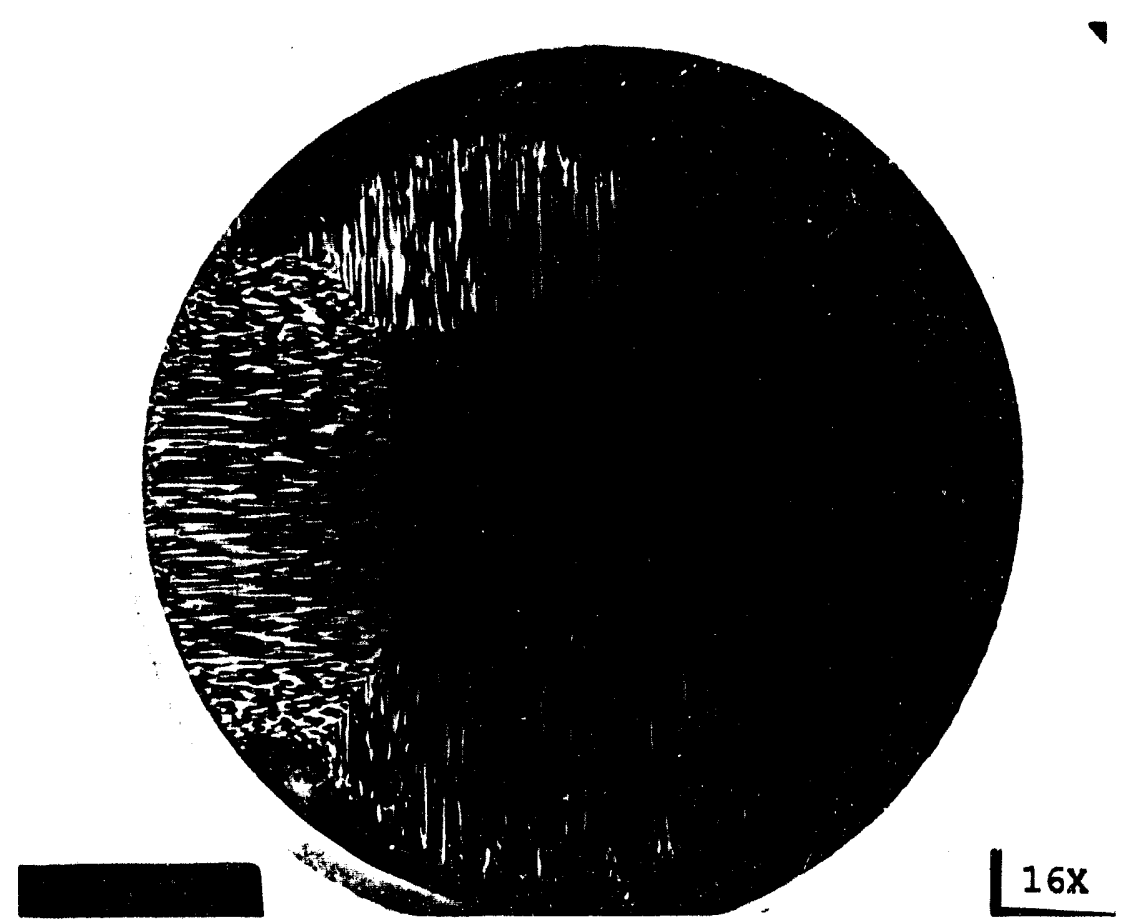

Figure 80

(001) Surface View GTA Rotational Weld of Two Melt Patterns 


\section{Comparison of (001) and (011) Profilometry}

Topographic analysis was performed on two GTA 758 helium and 25 argon rotational welds of different orientations. Welding a single crystal on the (001) and (011) planes produced a pronounced ripple effect on the surface of each solidified weld pool. A topographic analysis for each of the two samples was performed in order to determine the amplitude and frequency of the observed undulations. For each plane there were four scans made from the edge to approximately the center of the weld. Figure 81 shows the basic pattern for the (001) surface for one quadrant of the weld from the $0.6 \mathrm{~mm}$ position to the $2.2 \mathrm{~mm}$ position of the scan. In this figure $R a$ is the arithmetic mean of the departures of the roughness profile from the mean line. $\mathrm{Rq}$ is the root mean square value of $\mathrm{Ra}$. Rt is the product of the maximum depth of the profile plus the maximum height of the profile. Finally, Rsk is the skewness or a measure of the symmetry of the profile about the mean line. In this pattern it was possible to count the number of peaks to determine the peaks per millimeter, 17 peaks/mm for the represented scan. It can be seen that the distance at which the measurements were taken was 1.6 millimeters and the total height, peak-to-valley, of the scan is 1.13 microns. An expanded profile of two scans which isolates the entire scan to a distance of 0.25 millimeters can be seen in 

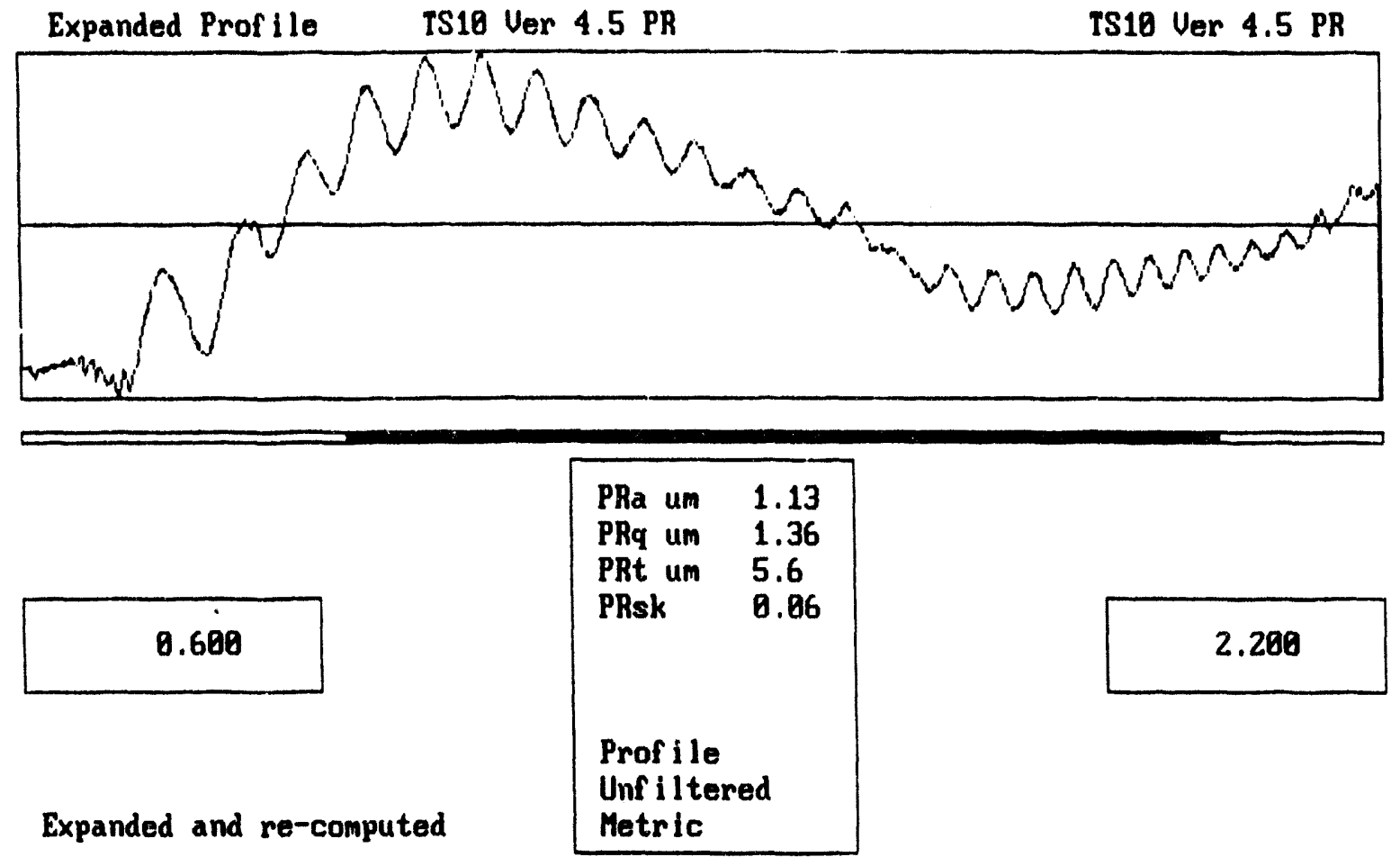

Figure 81

Profilometry Scan for the (001) Surface 
Figure 82. These expanded sections permitted the calculation of peak height verses the position in the scan of the peak. These figures demonstrate the periodicity and the amplitude of the surface peaks. From the data analysis [35] of the entire (001) profilometry scan, the frequencies and amplitudes of the undulations were determined (Figure 83 ). This showed that progression from the edge to the center of the weld resulted in an overall decrease in the amplitude of the undulations and an increase in the frequency. In each of the four scans, there was a section in which there was a rise in the amplitude followed by another decrease. The result gave a semi-sinusoidal shape to the amplitude-versus-position plots.

A section of the (011) nrofilometry was shown in Figure 84 . It is clearly seen that $t \quad s$ are not as visible on this scan as in the (001) plane. Exparued sections of this scan can be seen in Figure 85. Even in the expanded section of the scans, the peaks are not well defined as compared to the (001) plane. Figure 86 represents the calculated data for the amplitude and the frequency of the ripples. The measurable peaks were fewer in the data analysis of this scan because the sensitivity of the profilometer did not capture the low-amplitude peaks. However, the same trends in the data could be seen as those found for the (001) plane where a decrease in the amplitude of the peaks occurred in going from the edge to the center of the weld. 

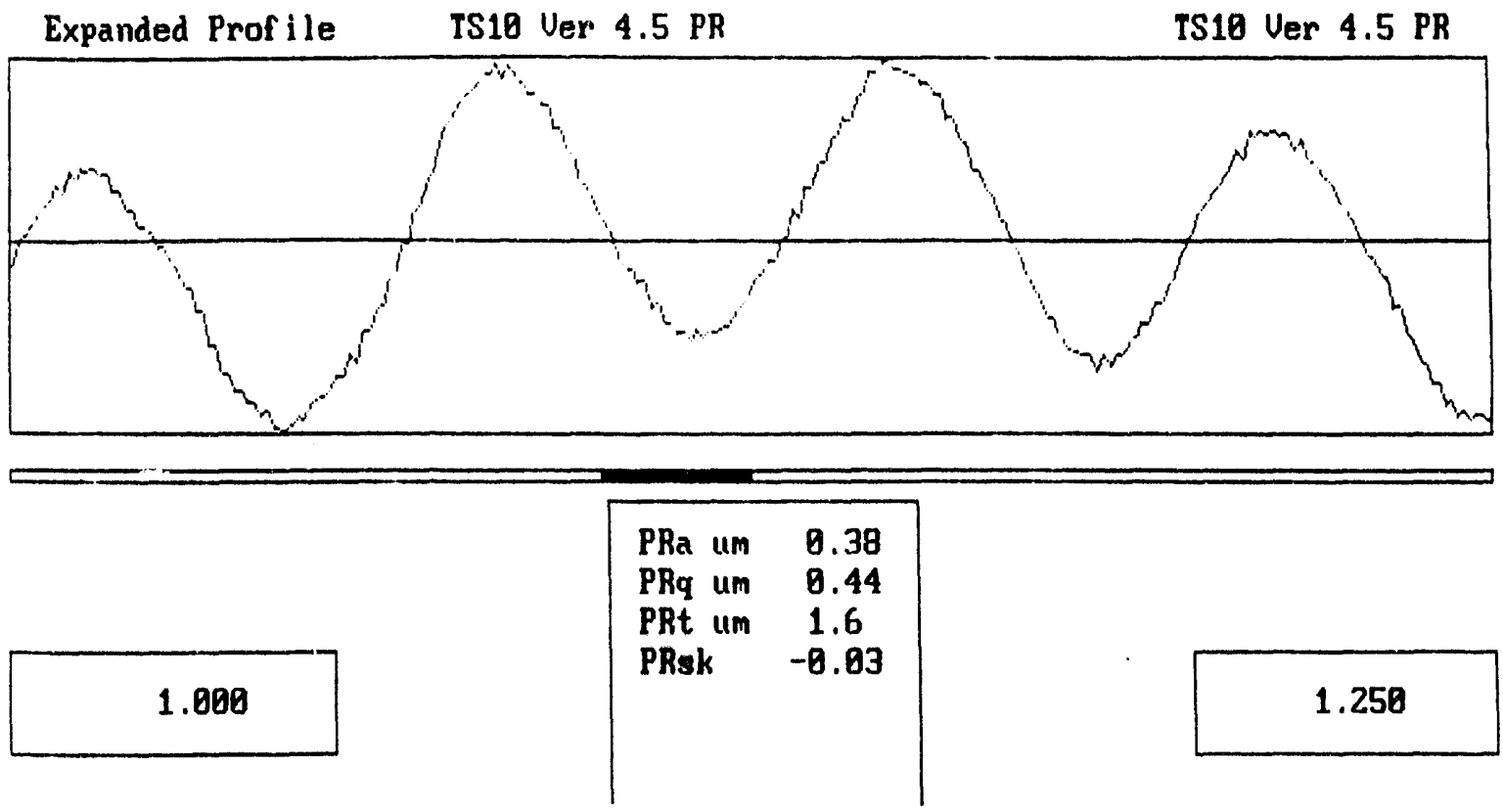

Expanded Prof ile

IS10 Ver 4.5 PR

TS10 Ver 4.5 PR
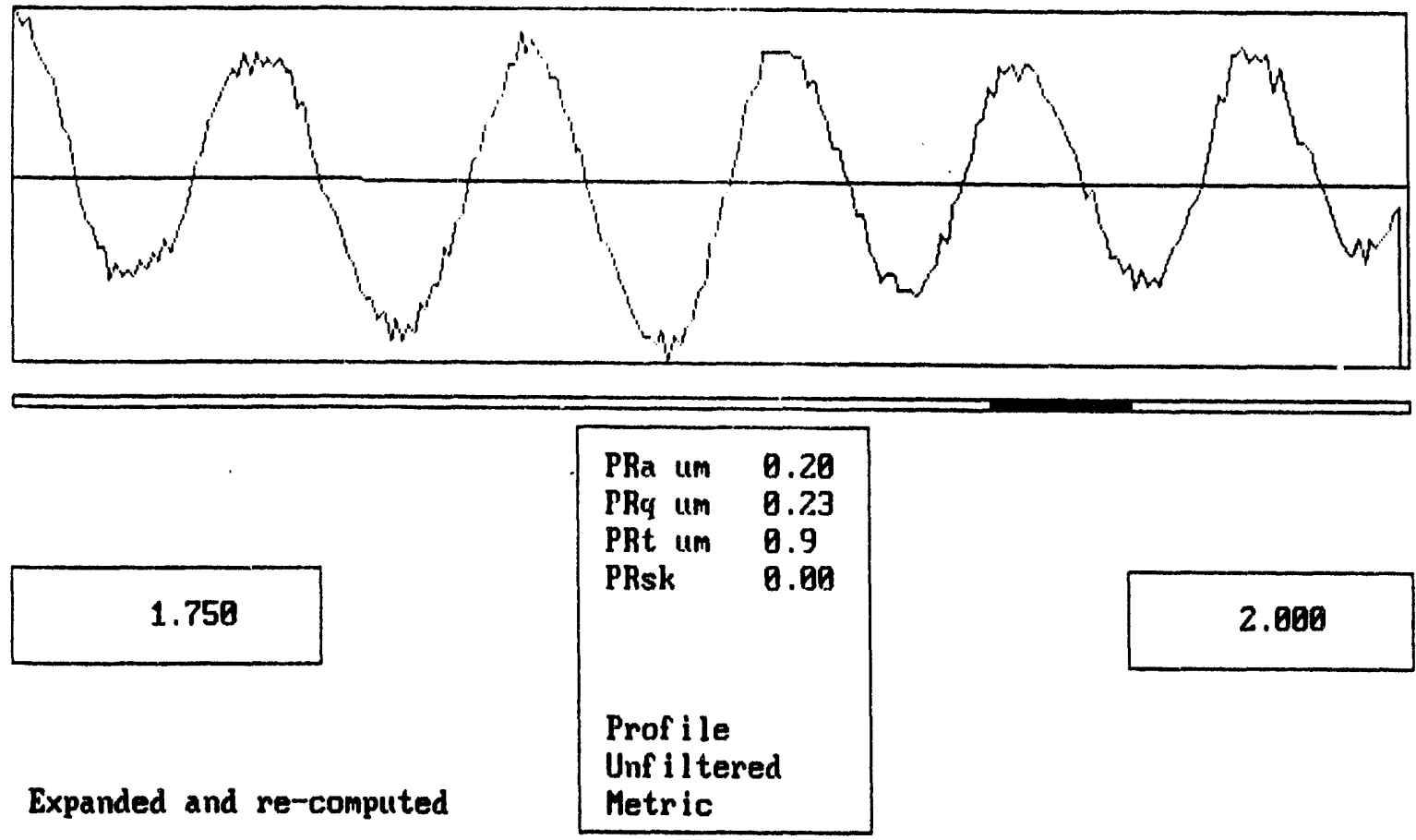

2.800

Figure 82

Expanded Scans (001) Orientation 


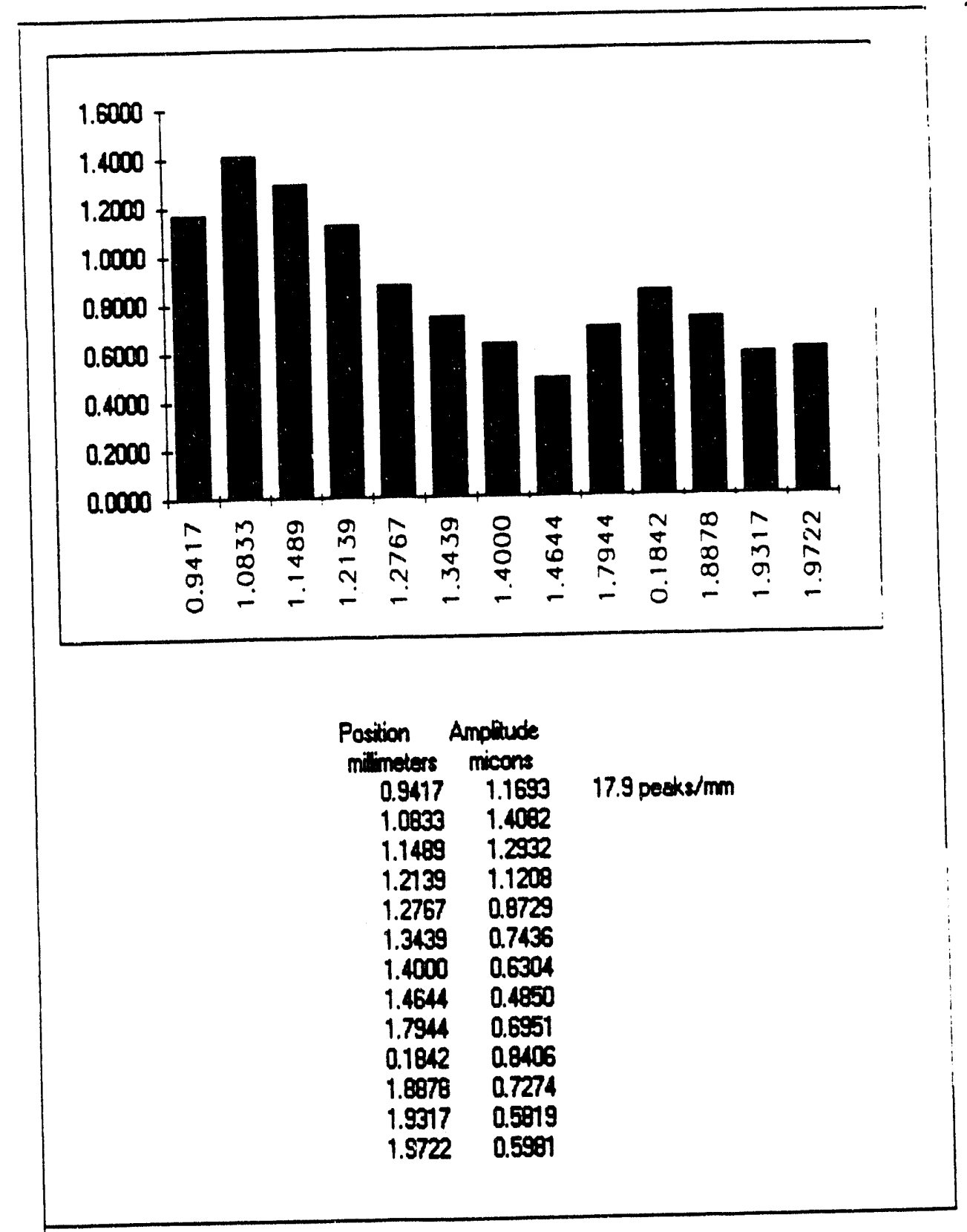

Figure 83

Data Plot of Amplitudes Verses Position (001) Plane 


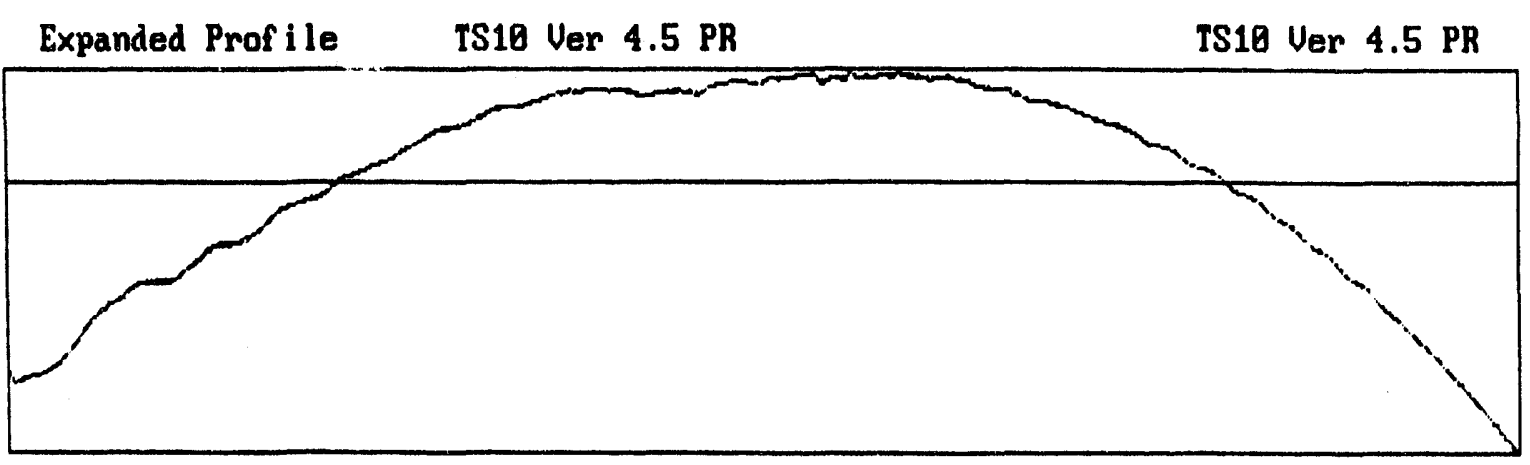

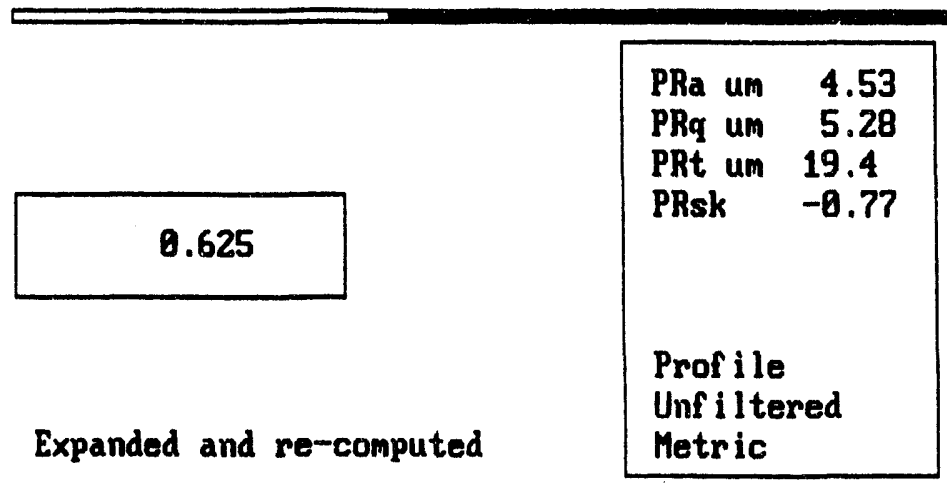

2.425

F1gure 84

Profilometry Scan for the (011) Surface 

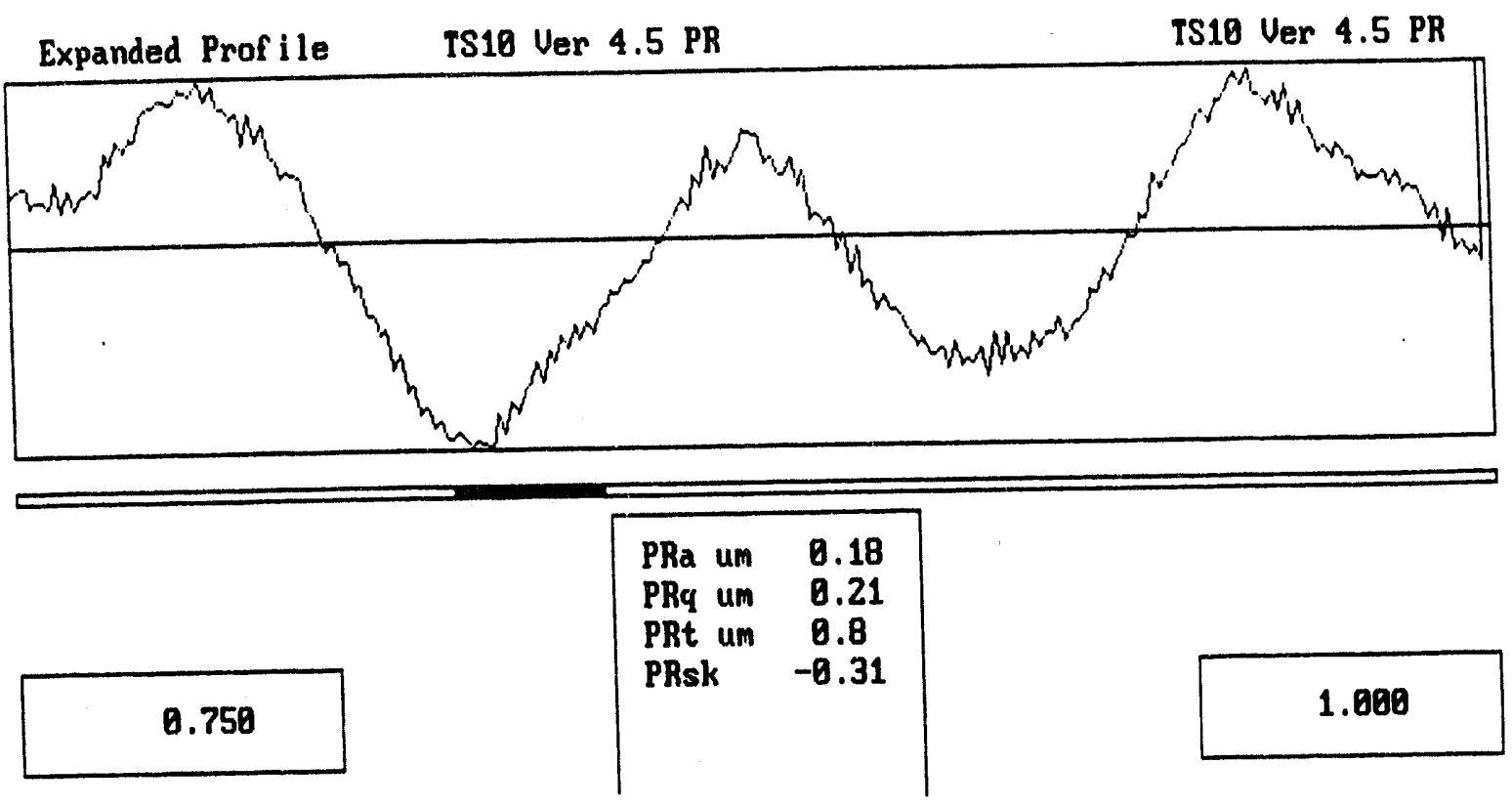

1.080

Expanded Prof ile

TS10 Ver 4.5 PR

TS10 Ver 4.5 PR
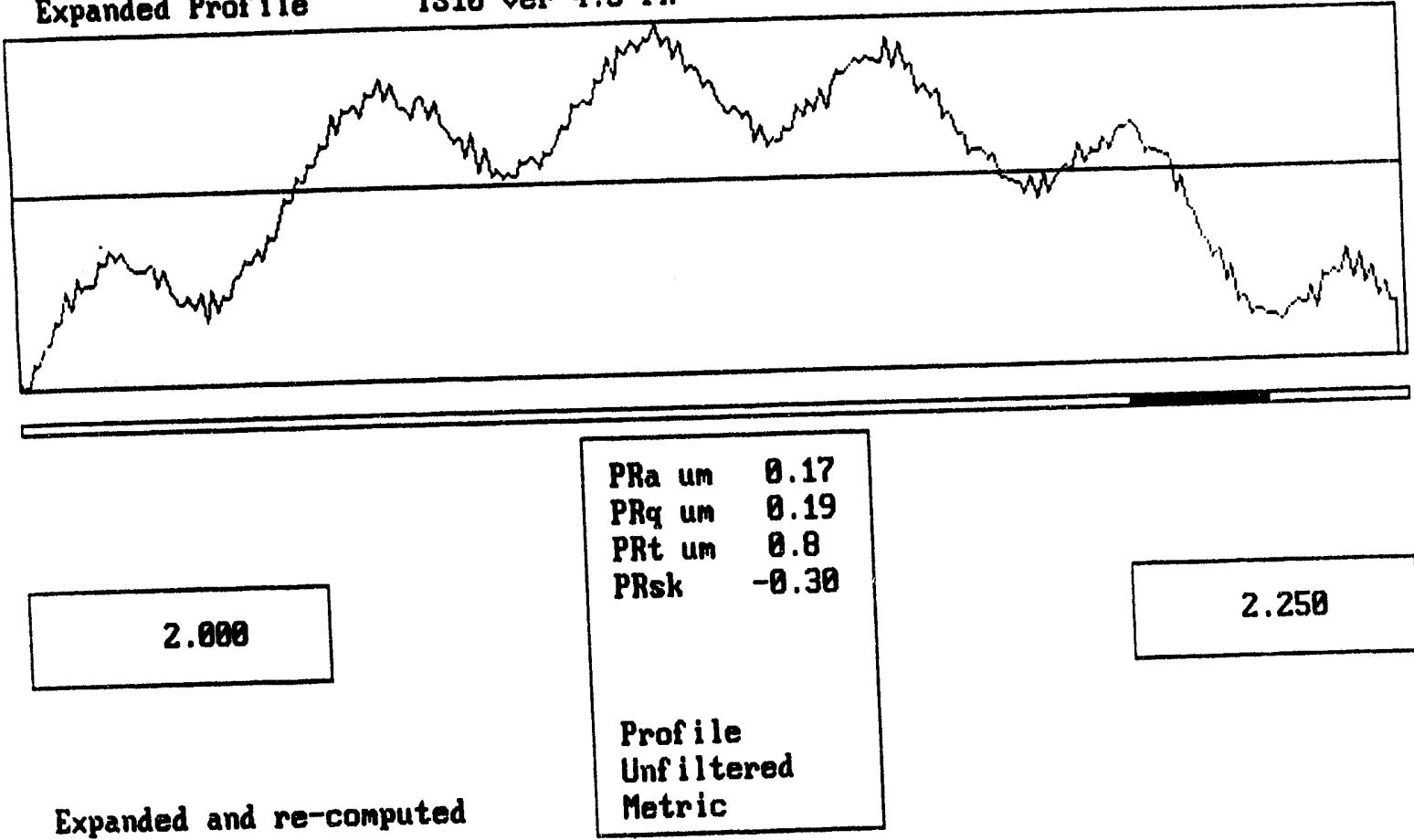

2.250

Figure 85

Expanded Scans (011) Plane 


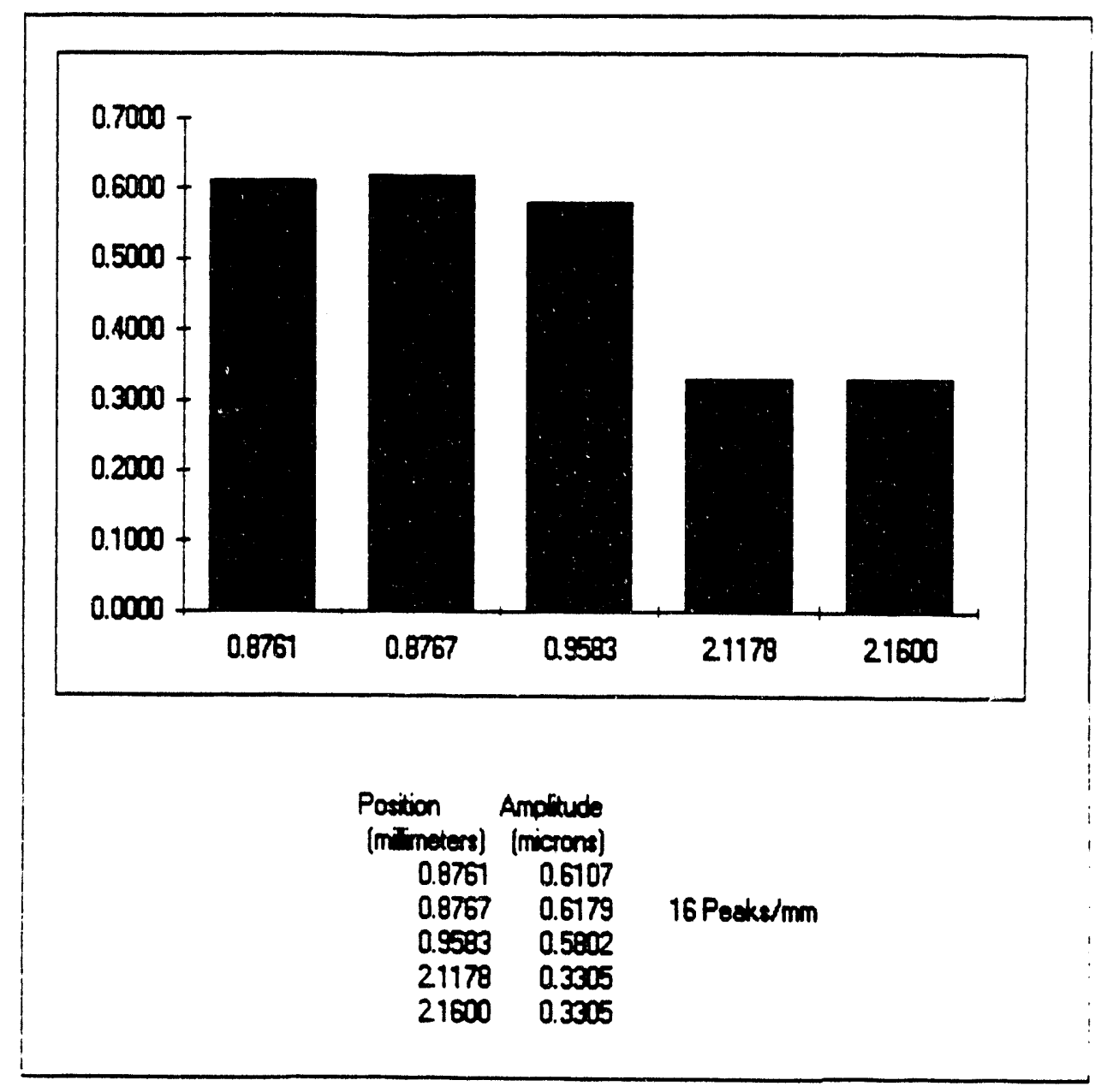

Figure 86

Data Plot of Amplitude Verses Position (011) Plane 
Furthermore, the frequency of the ripples increased as a translation from the edge of the weld to the center.

\section{Overlapped spot Helds}

Overlapped GTA spot welds were performed on two separate planes, (011) and (111). These welds revealed that the microstructure was not governed by the existing microstructure produced by the first weld, but by crystallography of the plane. Figure 87 illustrates an overlapped spot weld on a (011) plane

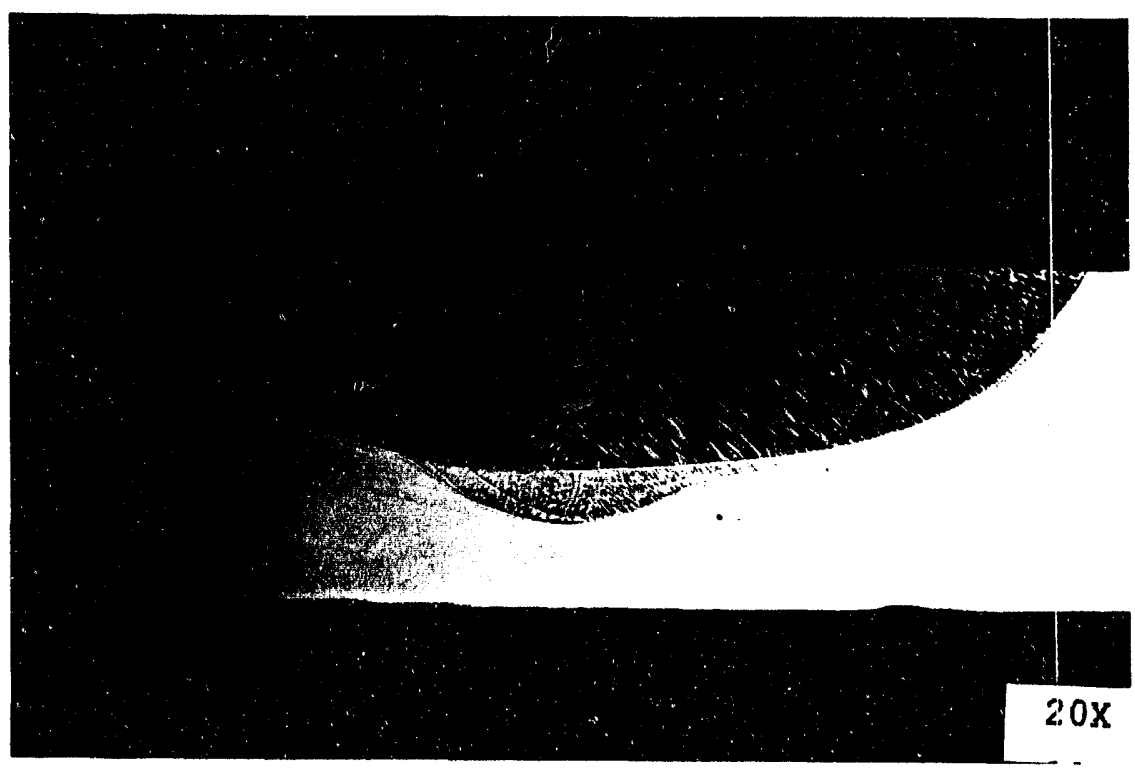

Figure 87

(011) Plane Stationary GTA Overlapped Weld [100] Transverse View 
with a [110]-direction transverse view. The microstructure is that expected for this plane as described in the previous discussions. Figure 88 gives a higher-magnification view of the lower section of the weld where both of the weld pools were, evident. The first weld was the deeper-penetration weld. The [001] and [010] dendrite union was in two distinct areas in the weld microstructure. It appears that in the second weld the centerline was moved to the left of the first weld. If the microstructure of the second weld depended on the microstructure

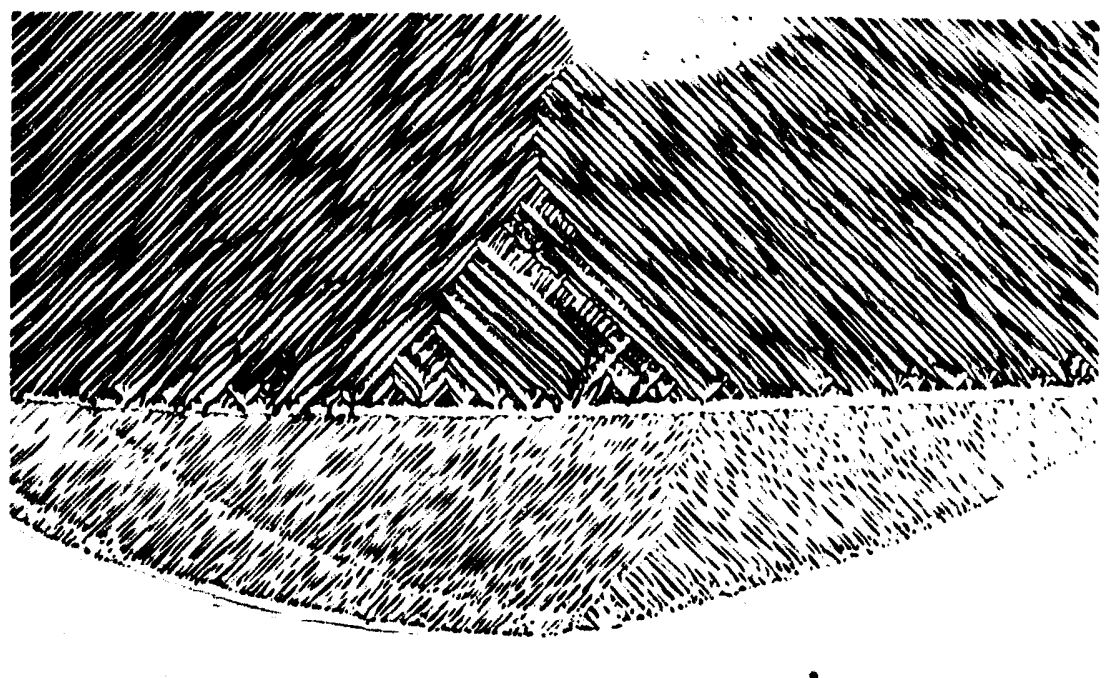

$64 \mathrm{X}$

\section{Figure 88}

(011) Plane Stationary GTA Overlapped Weld High Magnification of Bottom Section 
of the first weld, then the centerlines would be similar. In this figure, it is clearly seen that the microstructure of the second weld is independent of the microstructure produced by the first weld. Because there are regions in the first weld where there were [001] dendrites and in the second weld the dendrites were [010] dendrites. Therefore, the second weld microstructure was not determined by the previous weld, but by the crystallography of the sample.

The second set of overlapped welds were made on a (111) surface with a cross-section cut along the [110] direction (Eigure 89). The shallower-penetration weld was the first weld in this case. Figure 90 represents a high-magnification view of the left side of the overlap. The microstructural effects from this weld were not as obvious as those in the previous case because the growth patterns of this orientation were not as distinct as in the (011) plane. However, it was still supportive of the previous statements regarding the dendritic growth. The second weld depended on the crystallography and not the existing microstructure of the first weld as can be seen in the highermagnification photomicrograph. 

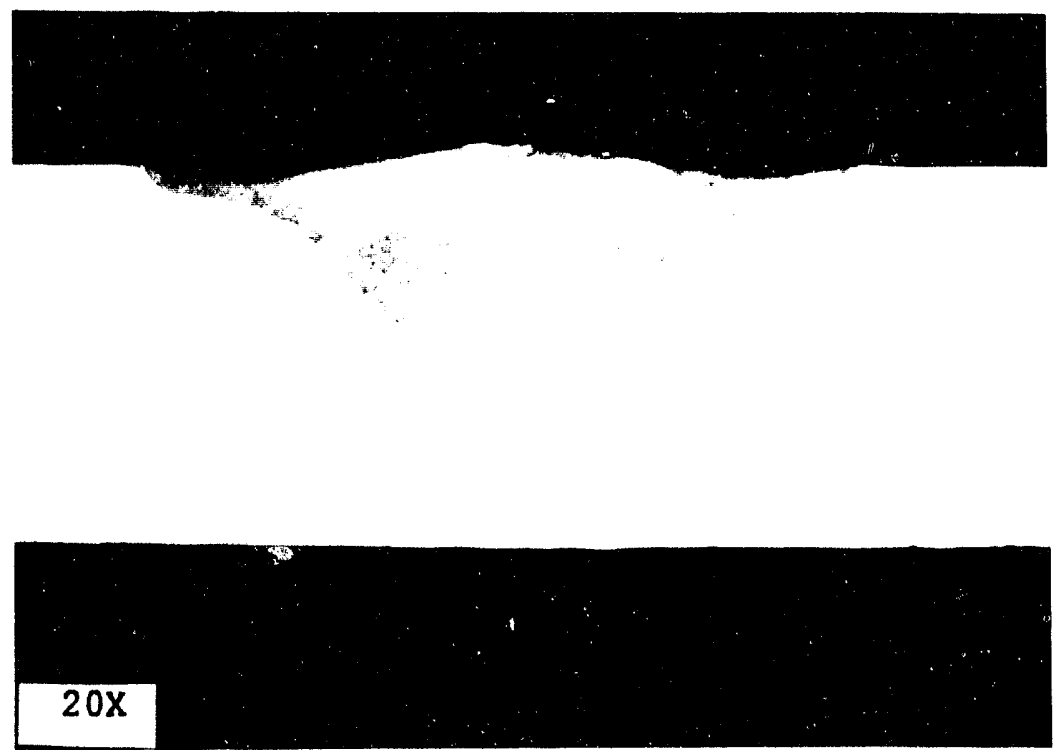

Figure 89

(111) Plane Overlapped GTA Rotational Weld [110] Transverse View

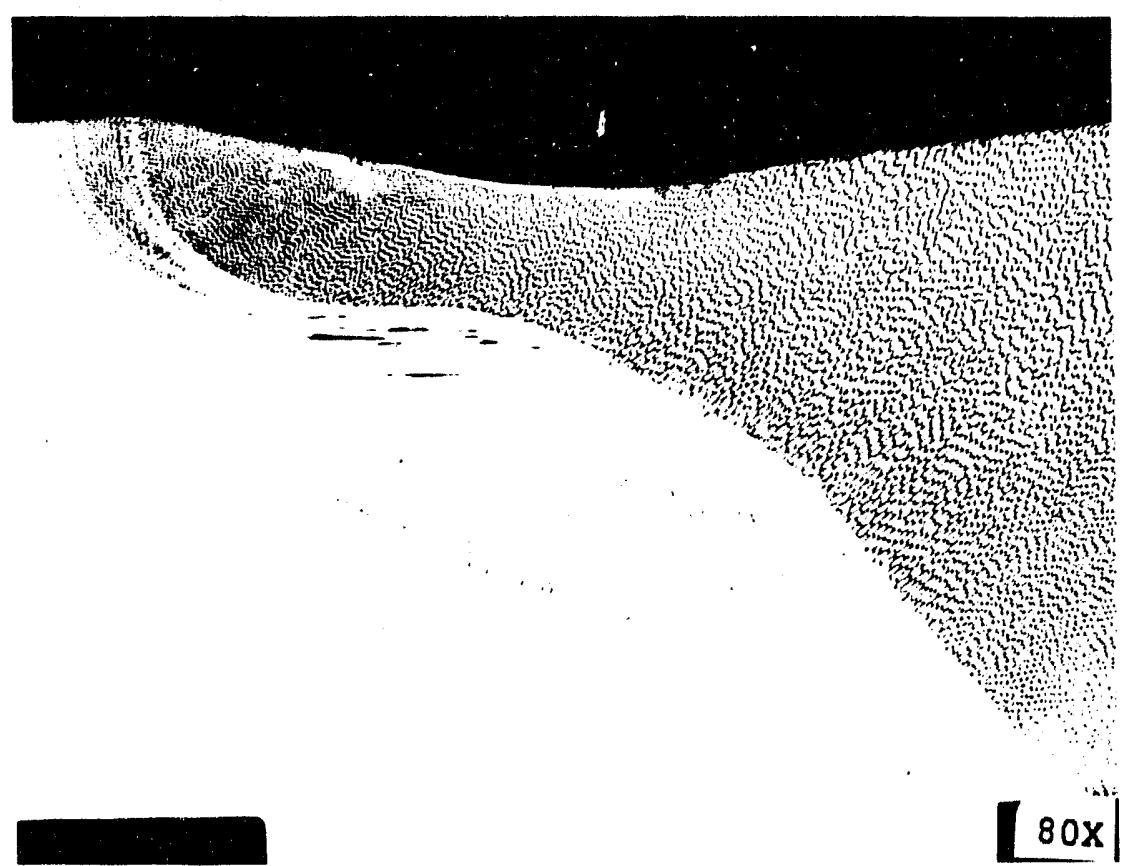

Figure 90

$\cdot 1$

(111) Plane Overlapped GTA Rotational Weld High Magnification 


\section{Comparison Between Gas Tungsten Aro 1008 Argon and GIA 758 Helium and 258 Argon}

The initial shielding gas used for the GTA welds was $100 \%$ argon. The resultant weld-pool shapes and microstructures are shown in the photomacrographs in Figure 91 . In studying the transverse views, a square-type melt penetration appeared in the microstructure which was unlike the rounded penetration achieved with the EB welds. It could be seen that the dendritic growth was along the [100] directions at the surface. Also, dendrites grew along the [001] direction in the center of the weld. The penetration depth was also very shallow, though the symmetry of the microstructure was still evident. The top surface shows the two fold symmetry formed from the dendritic growth. However, it is noted the shape of the weld pool is an oblong shape. The [100] dendrites growing across the surface and the [010] and [001] dendrite cross-sections are visible over the remainder of the weld microstructure. However, in this view, the "egg" shape of the overall weld pool is obvious. Therefore, the shielding gas was changed to a mixture consisting of $75 \%$ helium and $25 \%$ argon $(75 / 25)$. Helium gas was introduced in order to increase the penetration depth without affecting the width of the weld. The argon remained in the mixture because it contributed to arc ignition. A trangverse and top views of the welds made with the 


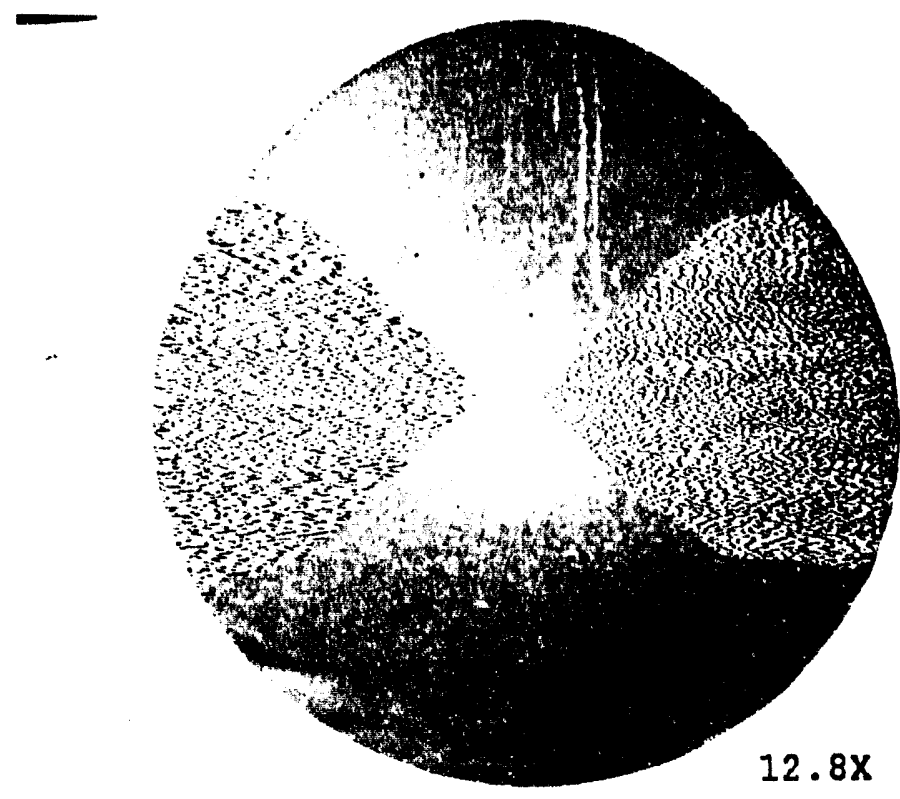

(a)
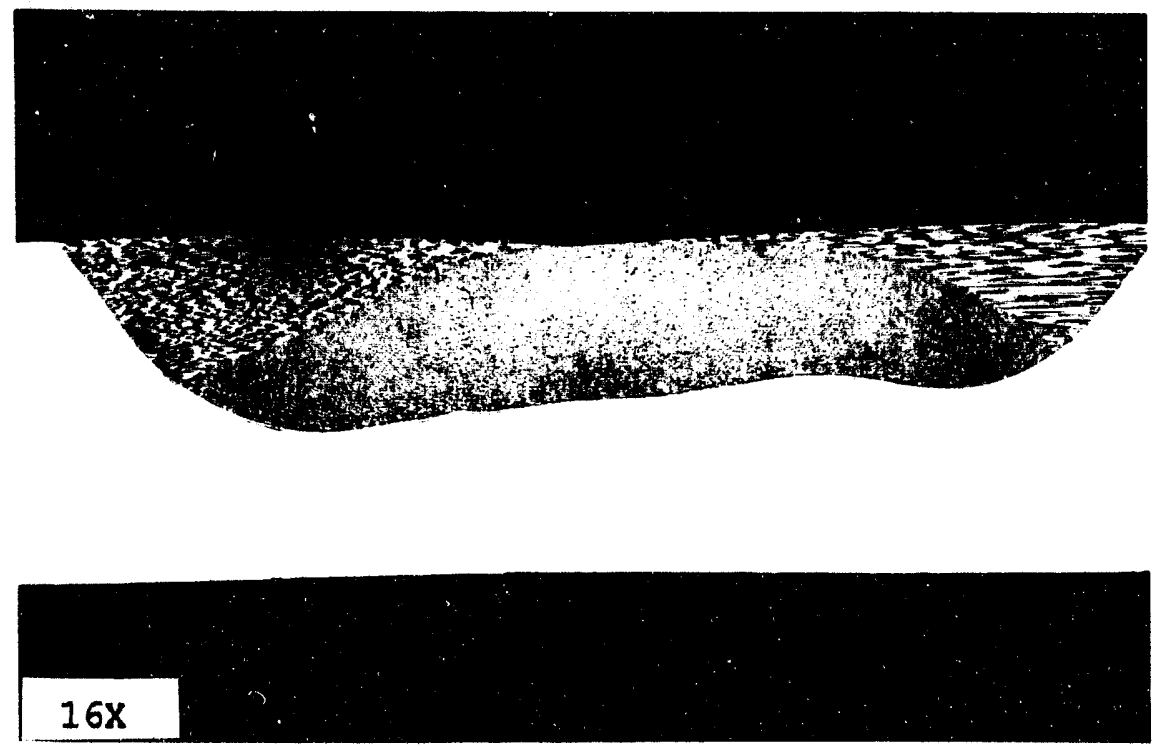

(b)

Figure 91

(011) Surface GTA 1008 Argon Stationary Weld
(a) Surface view
(b) [100] Transverse View 
$75 / 25$ gas mixture are shown in Figure 92 . These views show the sclidification pattern of the surface in the [100] direction parallel to the surface and the [001] and [010] directions coming at an angle to the surface. This is also seen in the [100] cross-sectional view. A comparison of the welds with the two gas mixtures revealed a similar microstructure for each case. The top views illustrated the microstructural similarities and the transverse views also showed some similarities. These views indicated that the $[100]$ dendrites were more frequent in the $100 \%$ argon welds, appearing throughout most of the weld depth. The "square-type" penetration of the weld pool shape resulted in dendrite growth mainly from the sides of the weld. Thus, for the 1008 argon, the top surface remained constant without the increase in concentration of the interior $\langle 100\rangle$ family of dendrites. The increased penetration of the $75 / 25$ gas mixture resulted in a rounded weld pool. This resulted in a decrease in the concentration of the [100] dendrites and an increase in the [010] and [001] dendrite concentrations. This evidence supports the previous arguments for the role of crystallography and weldpool shape in the development of the microstructure. 


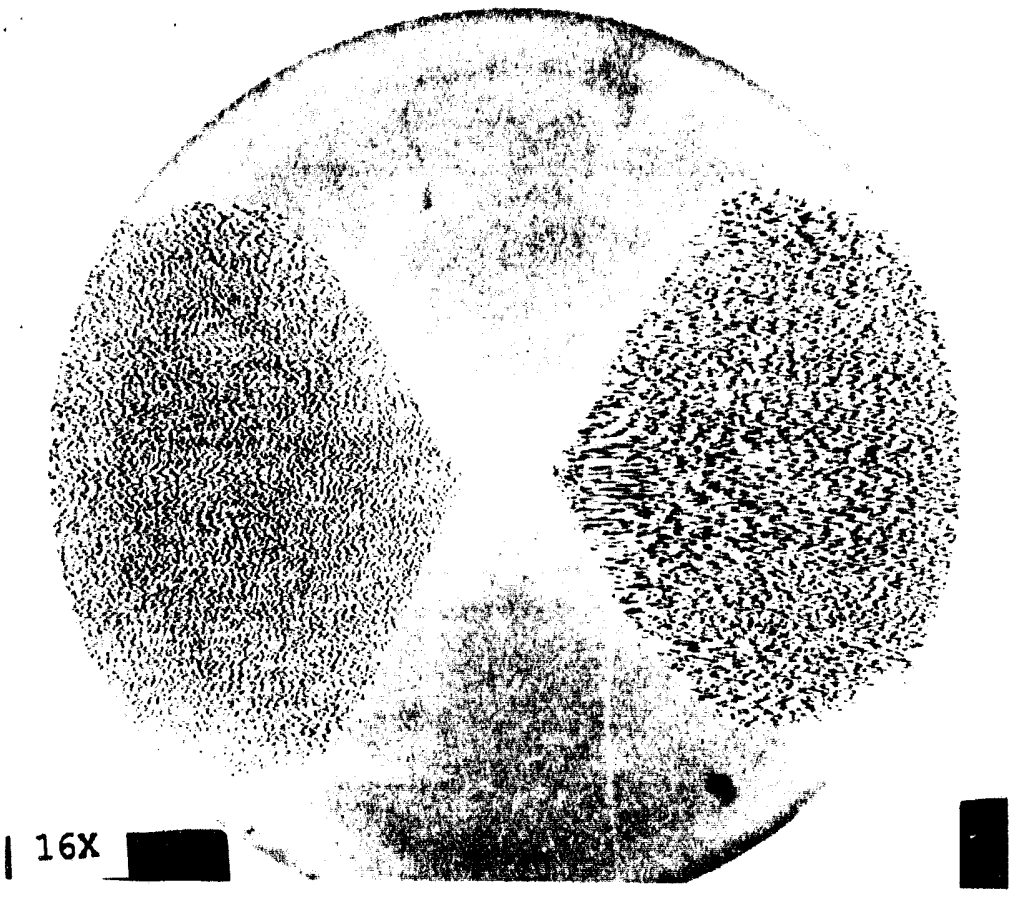

(a)
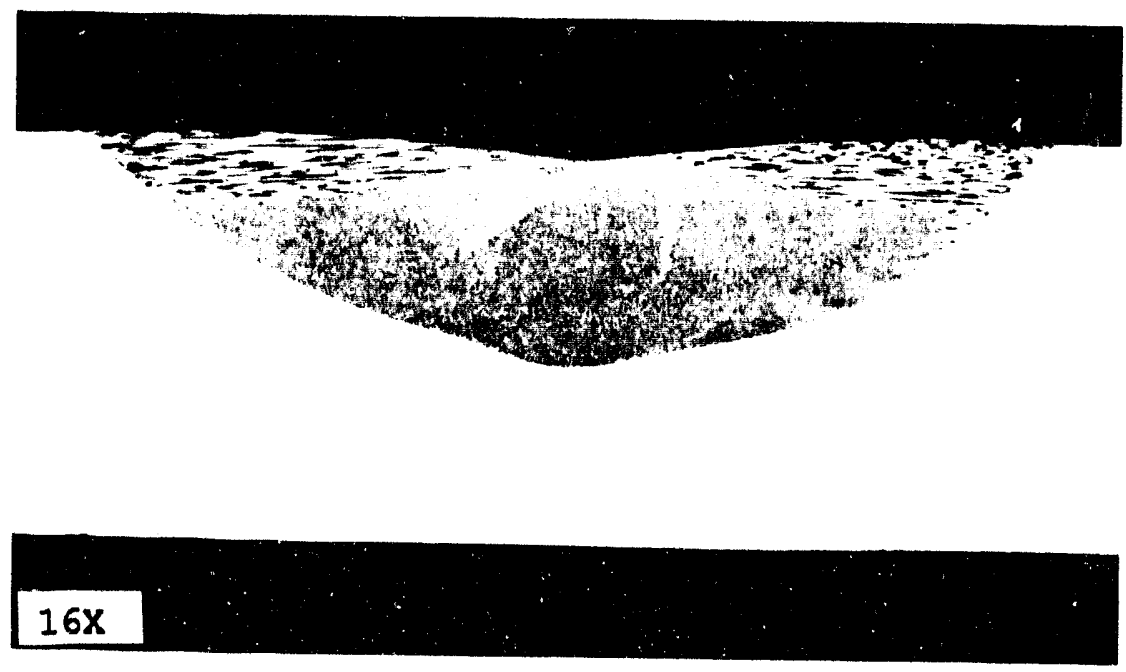

(b)

Figure 92

(011) Surface Stationary GTA $75 \%$ Hellum and 25\% Argon
(a) Surface View
(b) [100] Trangverse View 


\section{Electron Beam Felds (001) surface}

\section{(001) Crystallographic orientation}

The crystallography of this surface appears schematically in Figure 93 with the preferred dendritic growth directions noted in the rectangular areas [33]. The crystallography for this set of welds is the same as described in the stationary GTA welding section for the (001) plane [34]. For this case of welding, the EB was used in vacuum to produce a partial-penetration stationary spot weld. From the transverse views at various angles and the surface "in-depth" views a three-dimensional reconstruction of the resulting microstructure was produced for an EB spot weld on the (001) plane.

Sectioned for Transverse Views: The welding configuration for the EB melting is schematically shown with the primary directions, which lie along the periphery, as well as other directions for sectioning in Figure 94 . Figure 95 represents a three-dimensional reconstruction of the resultant microstructure with the accompanying transverse macrographs. Transverse views were sectioned along the two primary directions, [100] and [110]. Additionally, transverse views were obtained with a clockwise rotation about the $[100]$ direction of $9^{\circ}, 15^{\circ}, 18^{\circ}, 27^{\circ}$, and $36^{\circ}$. From these macrographs it was noted that the weld-pool shape changed with an increase in the angle of rotation about the [100] 


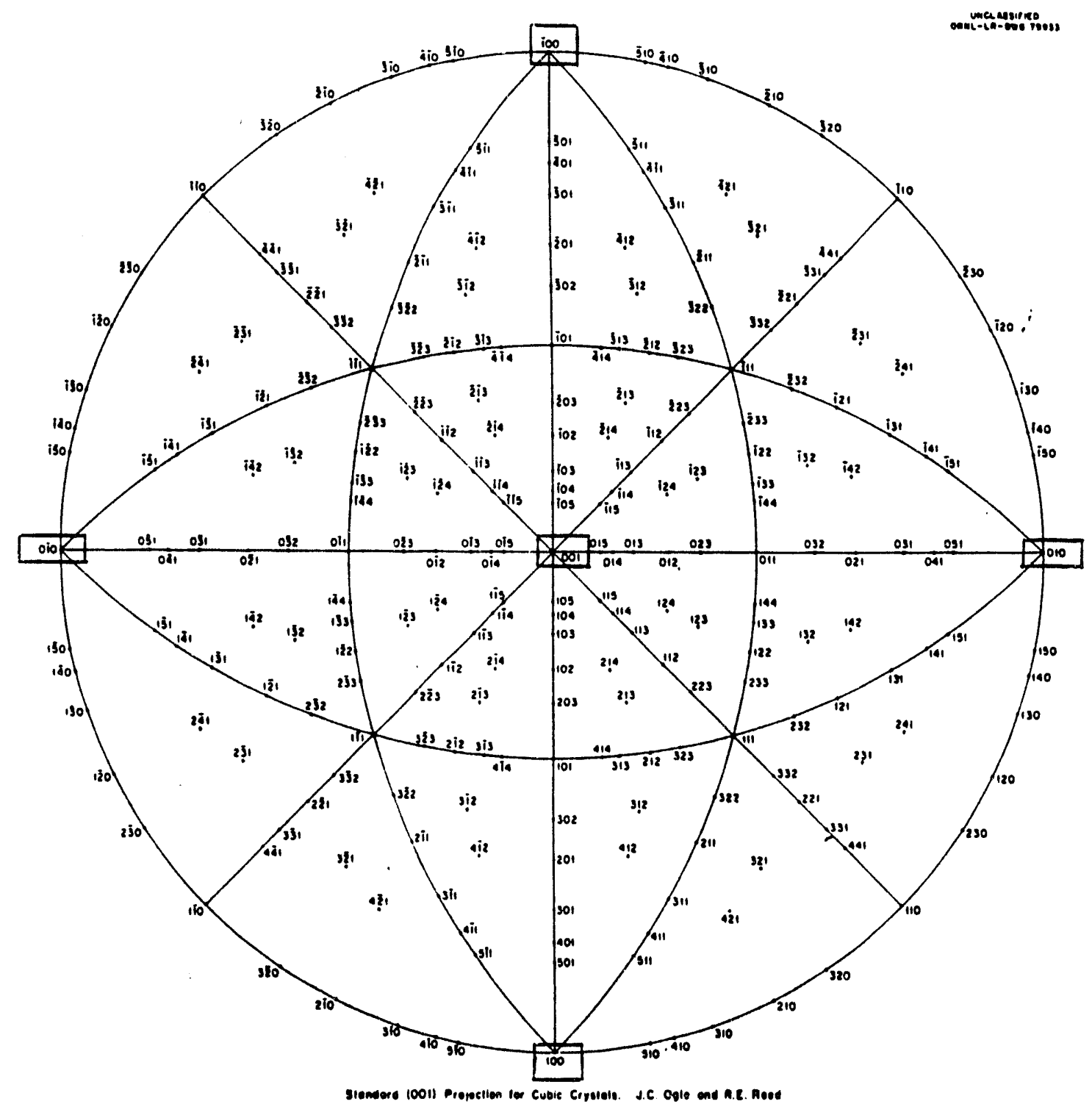

Figure 93

Stereographic Projection for the (001) Plane 


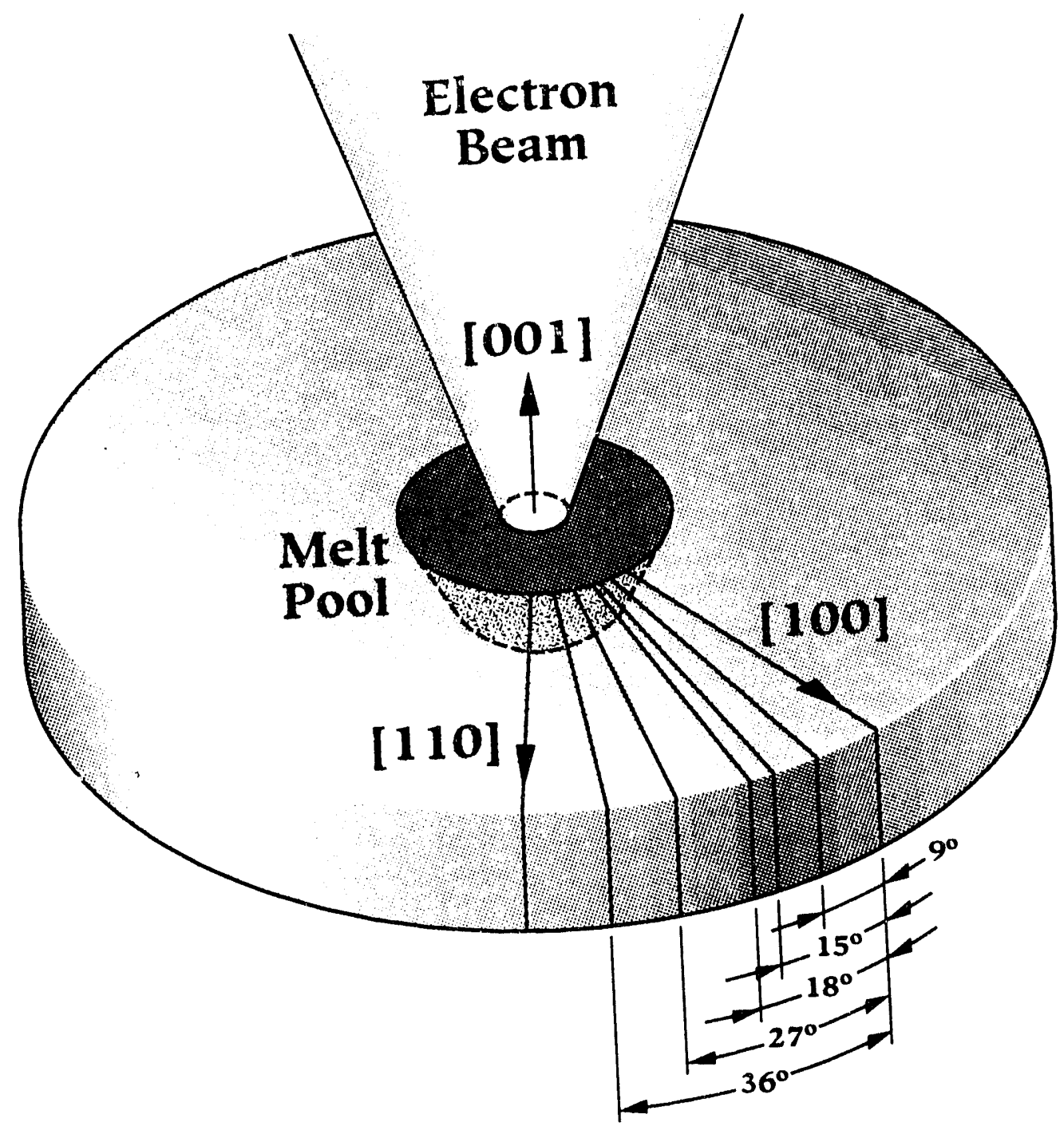

Stainless Steel Single Crystal (001) Surface

\author{
Figure 94
}

Schematic for (001) Plane EB Welding and Sectioning Configuration 


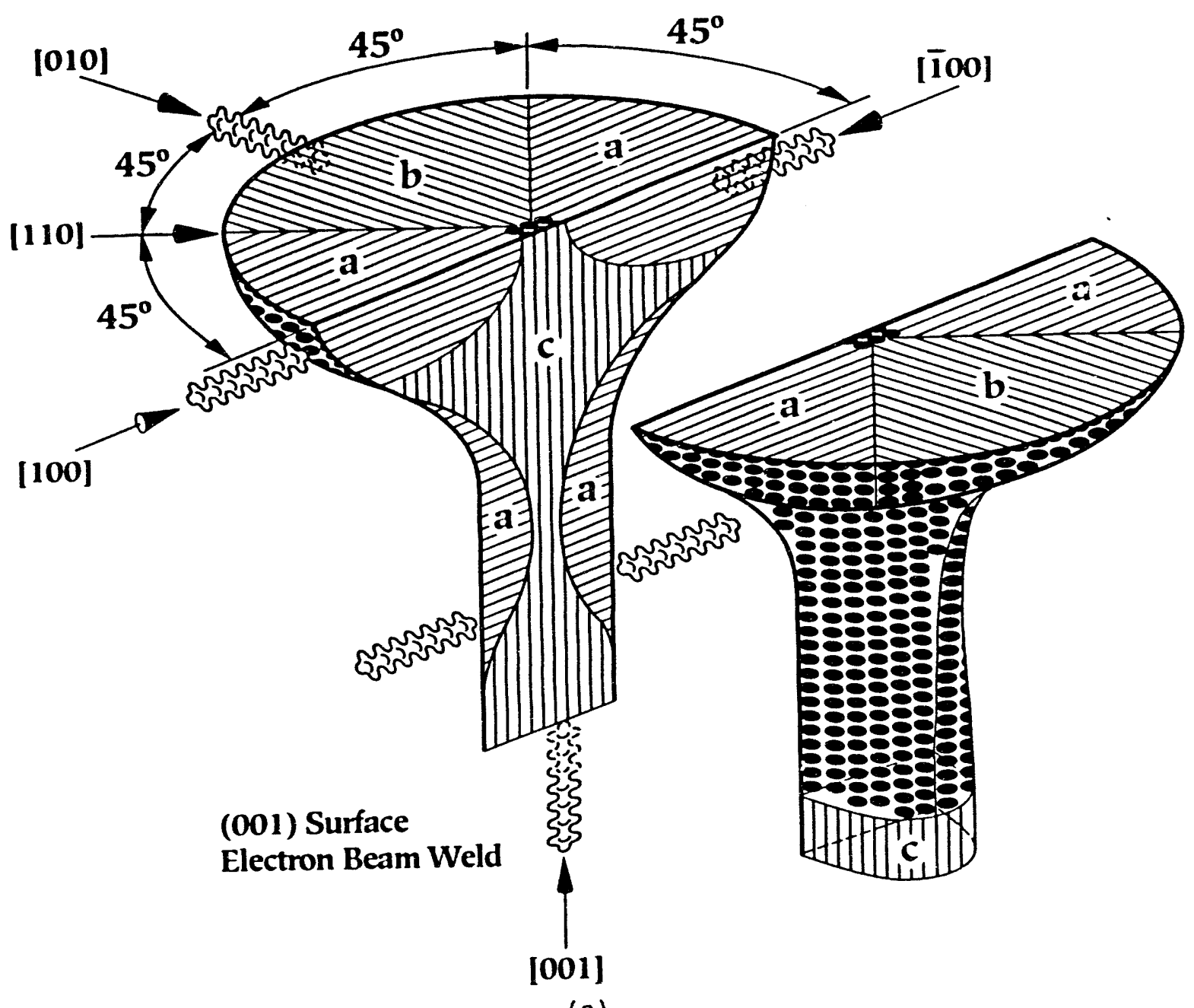

(a)

Three-Dimensional Reconstruction

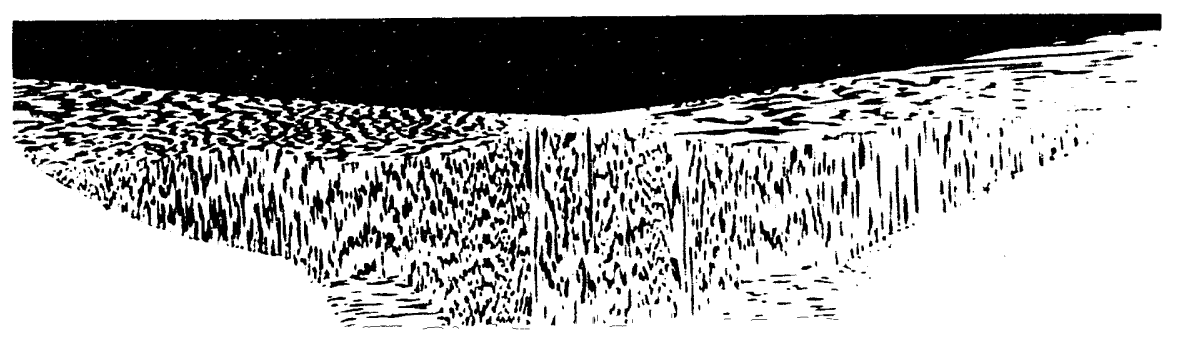




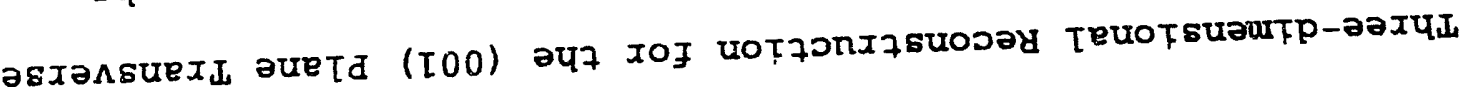

S6 จxח6Ṭ.

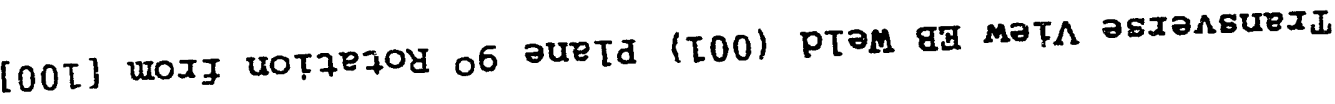

(2)

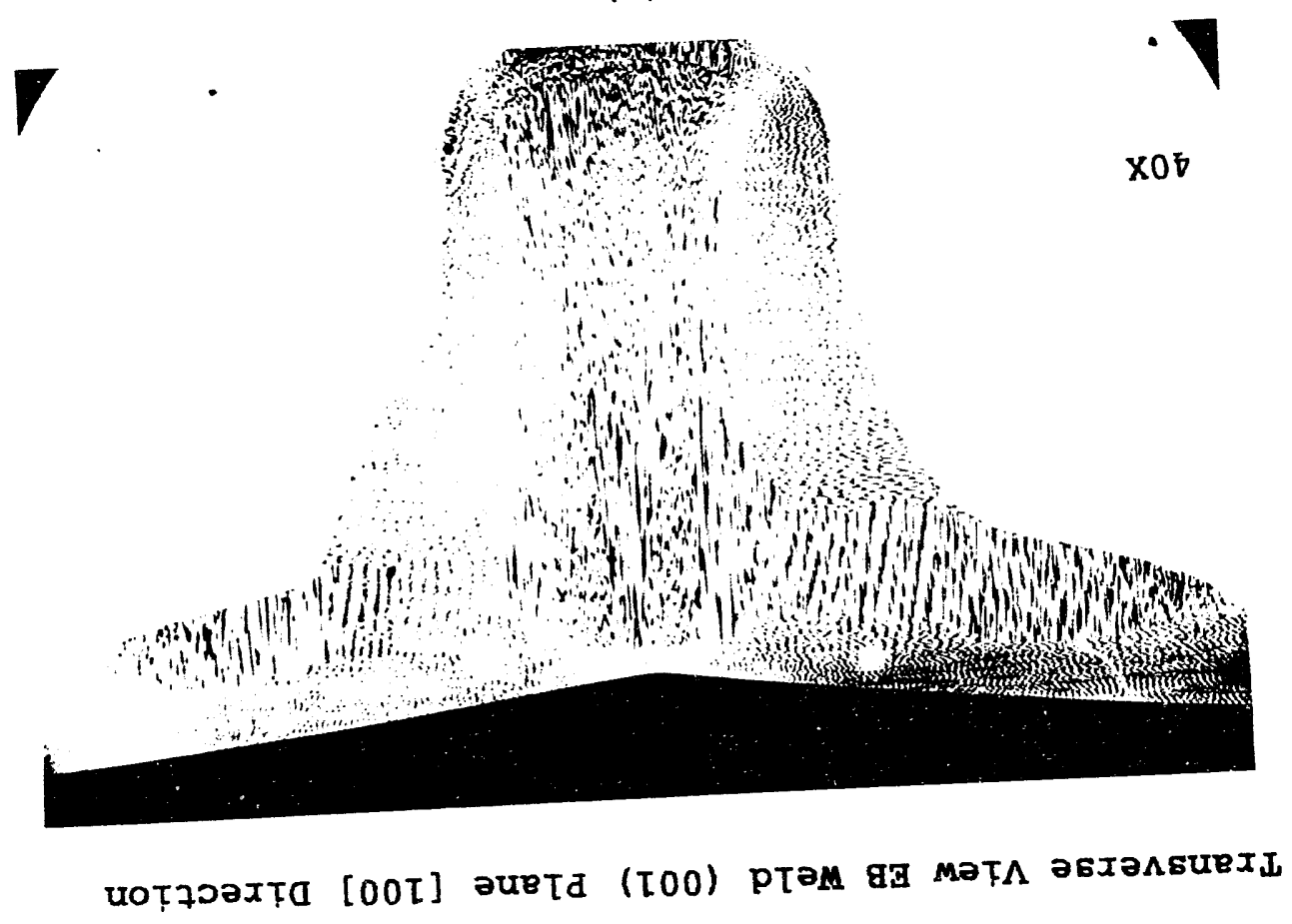

(q)

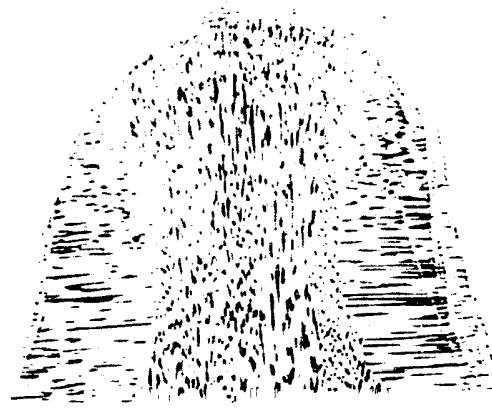

XOE 


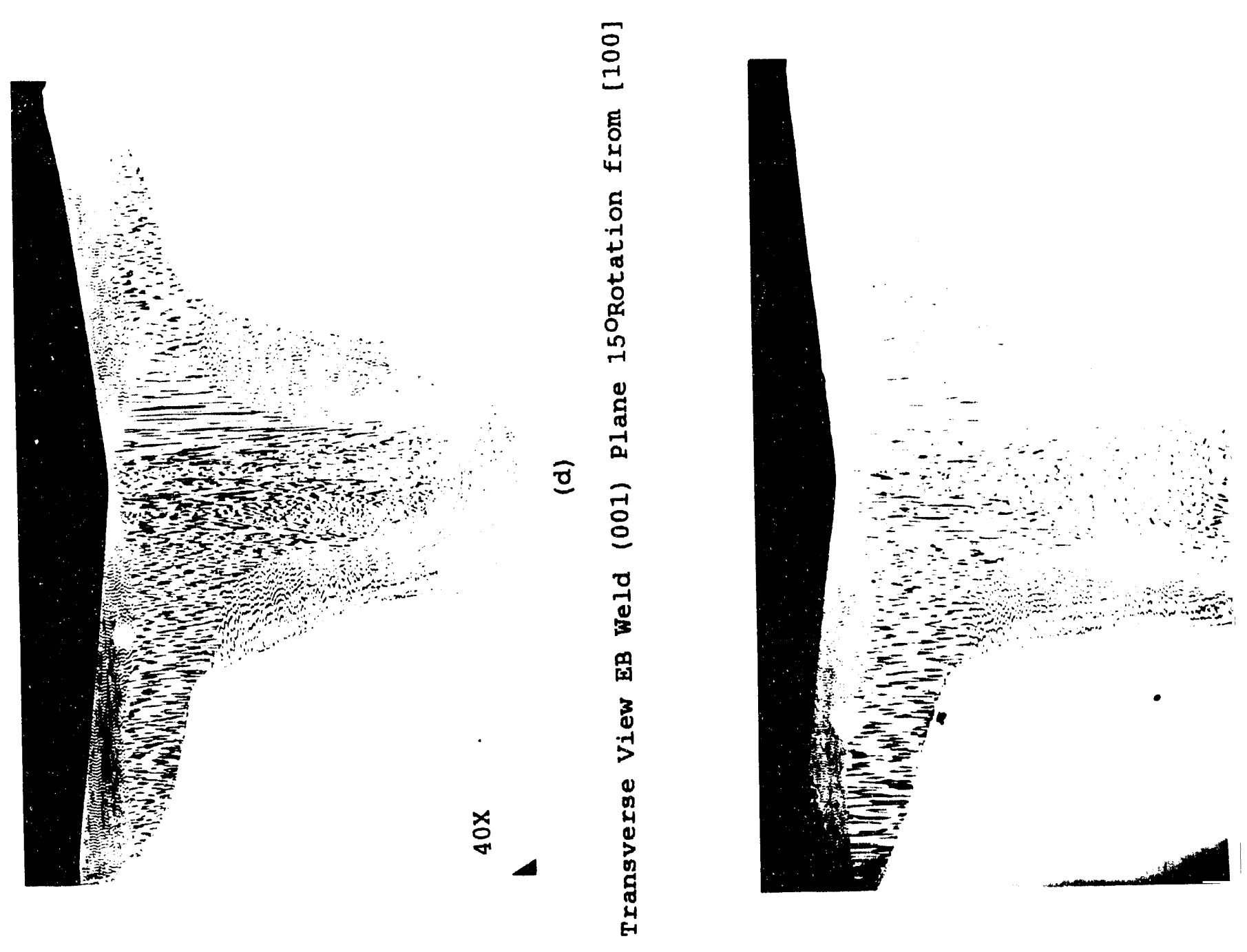


(e)

Transverse View EB Weld (001) Plane $18^{\circ}$ Rotation from [100]

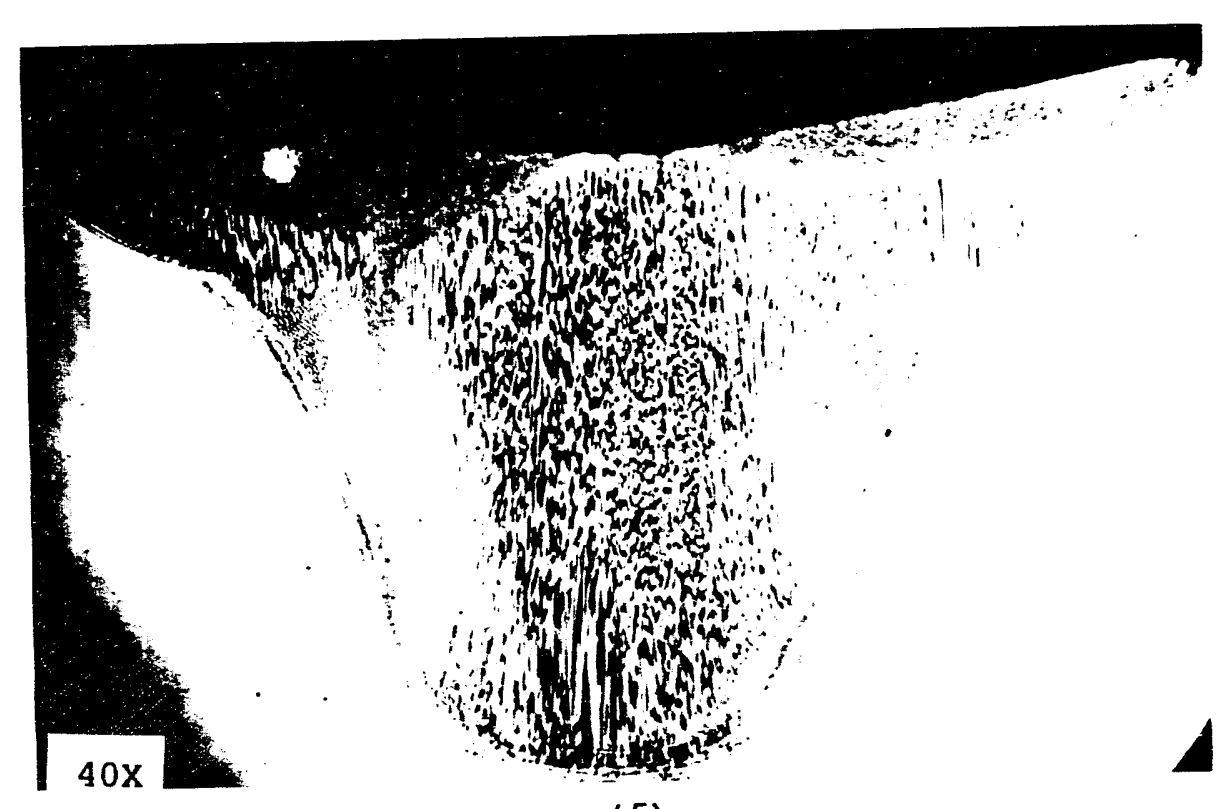

(E)

Trangverse View EB Weld (001) Plane $27^{\circ}$ Rotation from [100]

Figure 95 Continued 


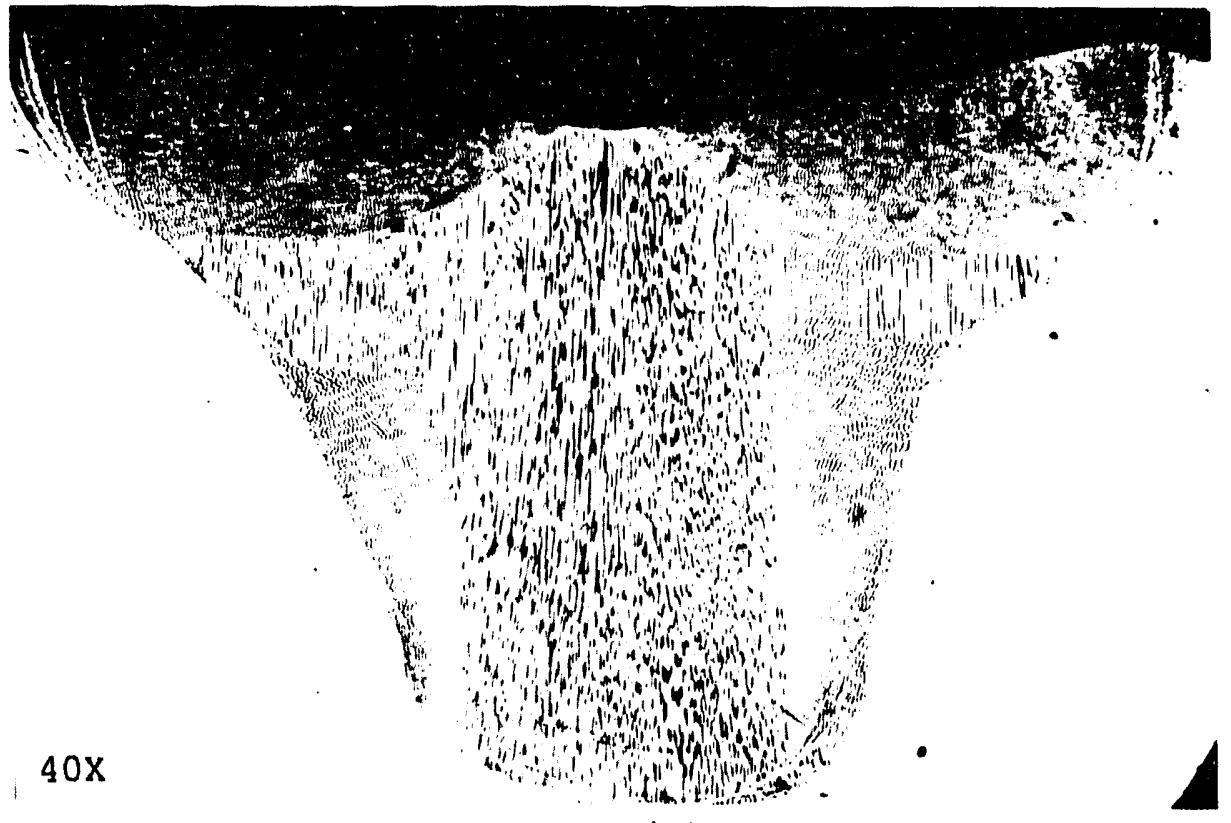

(g)

Transverse View EB Weld (001) Plane $36^{\circ}$ Rotation from [100]

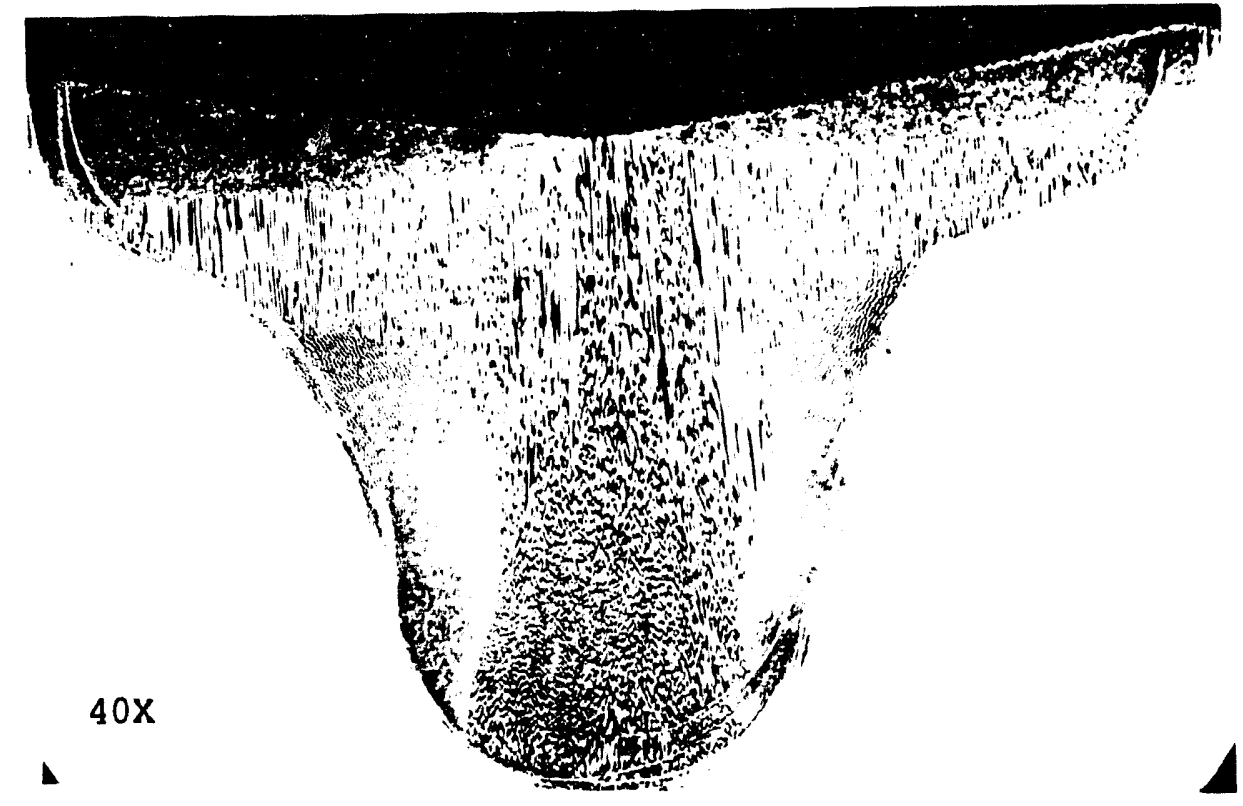

(h)

Transverse View EB Weld (001) Plane [110] Direction

Figure 95 Continued 
direction. This is illustrated by comparing the [100] (Figure 95b) and the $36^{\circ}$ transverse view (Figure 95g) in which the flat sections on the upper sides of the weld (ledges) were greatly reduced. The dendritic growth patterns also changed with this rotation. Again, a comparison of the [100] with the $36^{\circ}$ crosssections illustrates this fact. Near the top of the weld at the ledges in the [100] cross-section, dendrites grew in the [001] direction. However, in the same general area of the $36^{\circ}$ view dendritic growth was in the [100] direction. This change in dendrite growth is expected because the change in the weld pool shape favored different "easy growth" directions. Observation of the [100] transverse view explains the presence of a semi two fold symmetry which appeared in a top surface view. It could be seen in this view that the dendrites that grew in the [001] direction appeared only on the two ledges and resulted in a section of growth in the [001] direction. Whereas in the other transverse views, growth still occurred along the [100] and [010] directions.

The general solidification pattern can be explained by observing the three-dimensional reconstruction (Figure 95a). This shows the angle at which the dendrites grew along with the other primary directions. In areas "a", dendrites grew along the [100] directions in areas "b" along the [010] directions. Both of these growths then propagated from the edge to the center of 
the weld. In areas " $C$ ", solidification was along [001] direction from the base metal toward the surface of the weld. This pattern was slightly altered, decreasing in the [001] dendrites, as the ledges were reduced by increase in the [100] dendrites in these areas. The [100] transverse view explains the semi two-fold symmetry which appeared in the top-surface view.

Top Surface: For the top surface microstructural analysis, an "in-depth" set of top surface macrographs of the (001) plane are shown with a three-dimensional reconstruction of the resultant microstructure (Figure 96). From the surface photomacrograph in Figure $96 \mathrm{~b}$ it was noted that the microstructure had four fold symetry on the weld surface. The dendritic growth was along the [100] and [010] directions, and in the center of the weld, dendrites grew in the [001] direction. The solidification of the weld at the top surface proceeded from the edge of the weld toward the center as stated previously. It consisted of the $\langle 100\rangle$ family of dendrites meeting along the <110> directions. As the depth into the weld increased, there was an increase in the concentration of the [001] dendrites. This resulted in a semi-two fold symmetry (Figure 96c). This was caused by the "ledges" in the [100] direction as discussed previously. However, deeper into the weld, inhomogeneities in the $E B$ heating caused a change in the weld-pool shape, from circular to oblong, as shown in the above photomacrograph given 


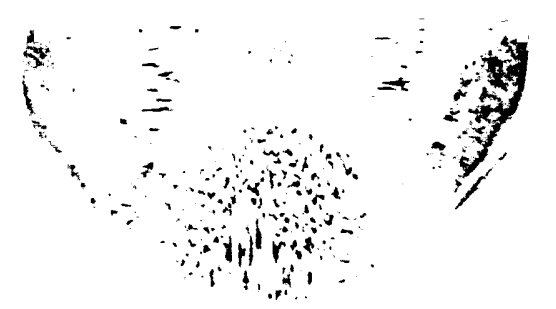

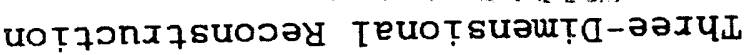

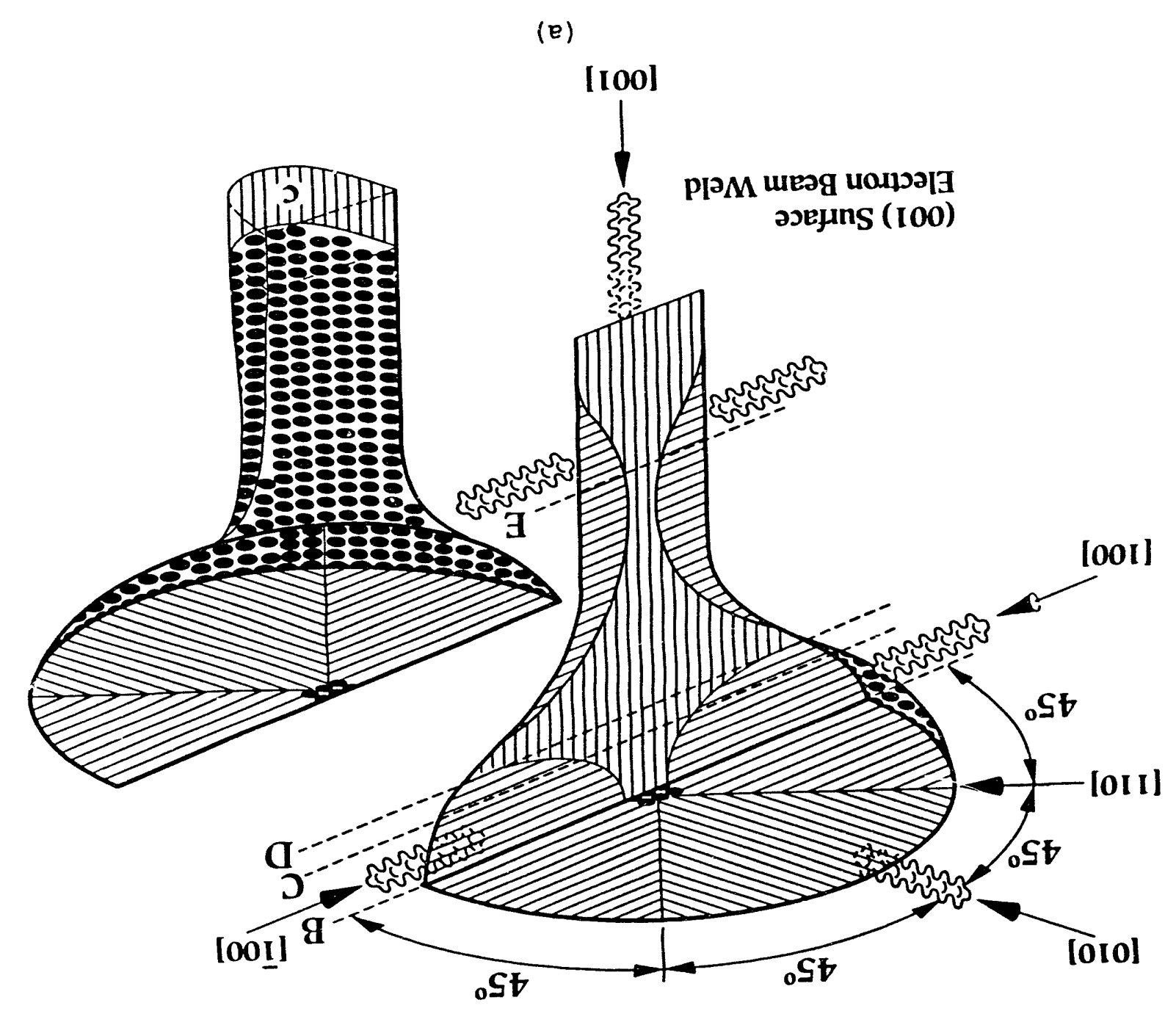




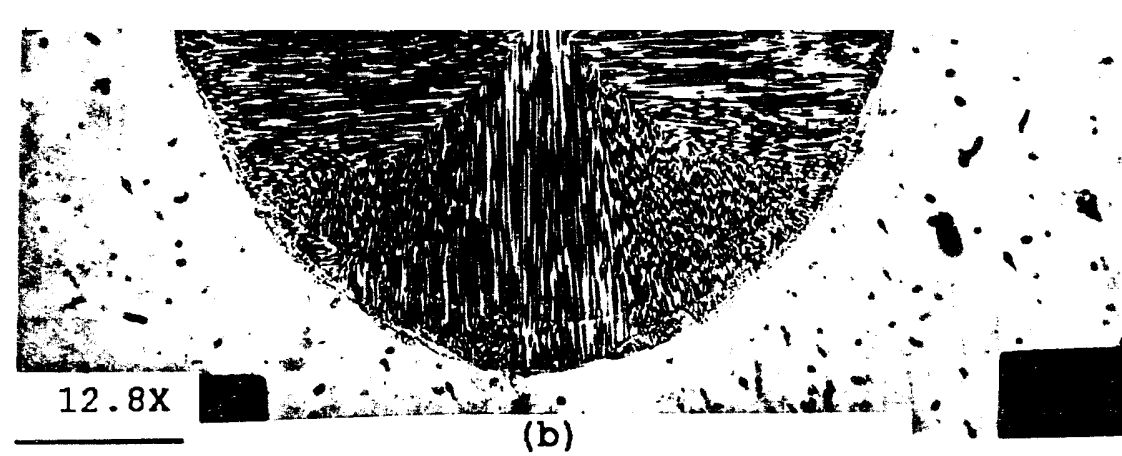

(001) Surface of EB Weld

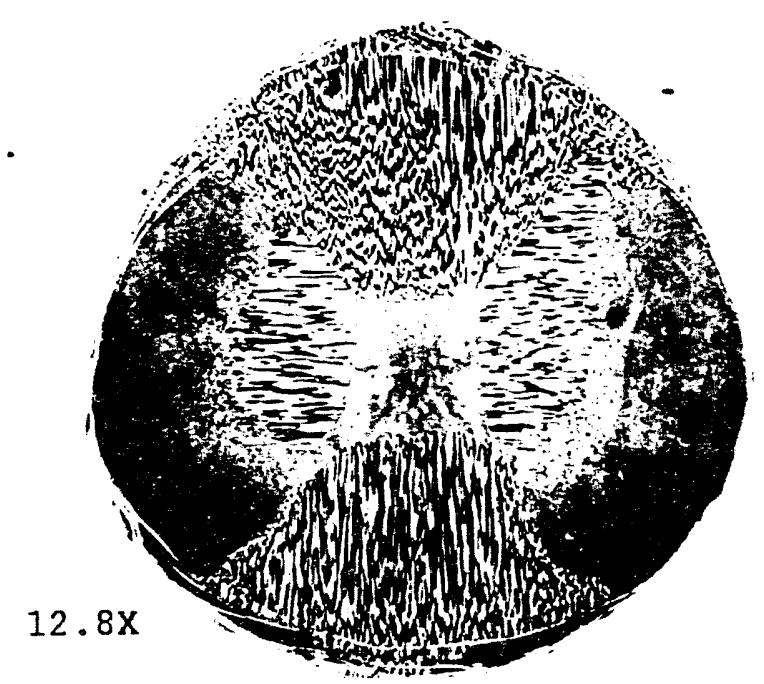

(d)

(001) Surface EB Weld

Increased Depth

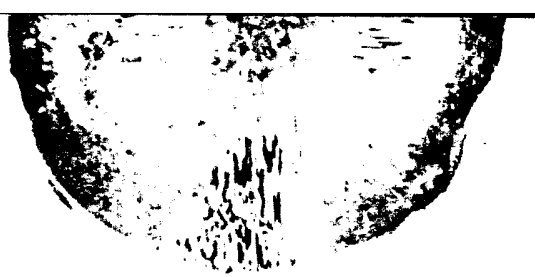

$12.8 \mathrm{x}$

(c)

(001) Surface EB Weld

Increased Depth

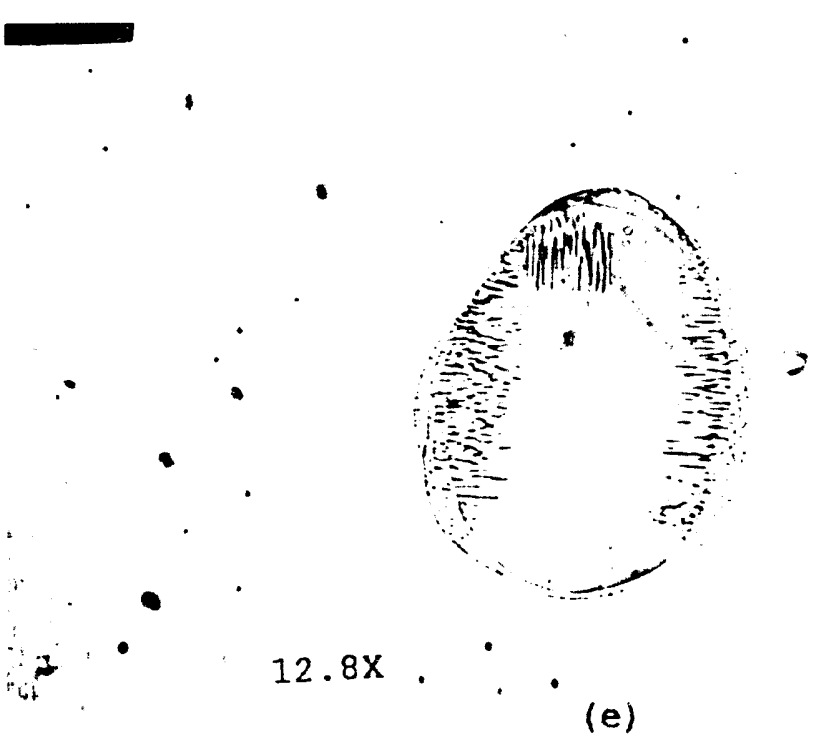

(001) Surface EB Weld

Near Bottom of Weld

Figure 96

Three-dimensional Recosntruction for the (001) Plane surface

Electron Beam Welded with Accompanying Photomacrographs 
in Figure 96d. The shape change was also noted on the sides of the transverse views. This shape change did not totally remove the symmetric microstructure, and a quasi four-fold symetry remained (Figure 96d). This demonstrates the major role of crystallography because the surface had only two favored growth directions for this plane, and growth was not completely dominated by one set of directions.

Several interesting facts were revealed as a result of the microstructural manifestations which occurred during these melting operations. If even a small deviation from the desired angle occurred during sectioning, the microstructure differed significantly. This is shown in a cut $2^{\circ}$ off of the [100] direction (Figure 97) compared with a cut exactly along the [100] direction. This deviation was confirmed by the Laue' backreflection $\mathrm{X}$-ray diffraction technique. This phenomenon shows the importance of having the correct angle during sectioning in order to obtain reliable micrographs. This shows that instead of dendrites visibly growing across the view there are only sections of dendrites.

High-magnification photomicrographs often clarified the solidification process. These are schematically represented in areas "A", "B", and "C" of Figure 98. Area "A" of Figure 98 is represented in Figure 99 where the dendritic growth in a [100] 


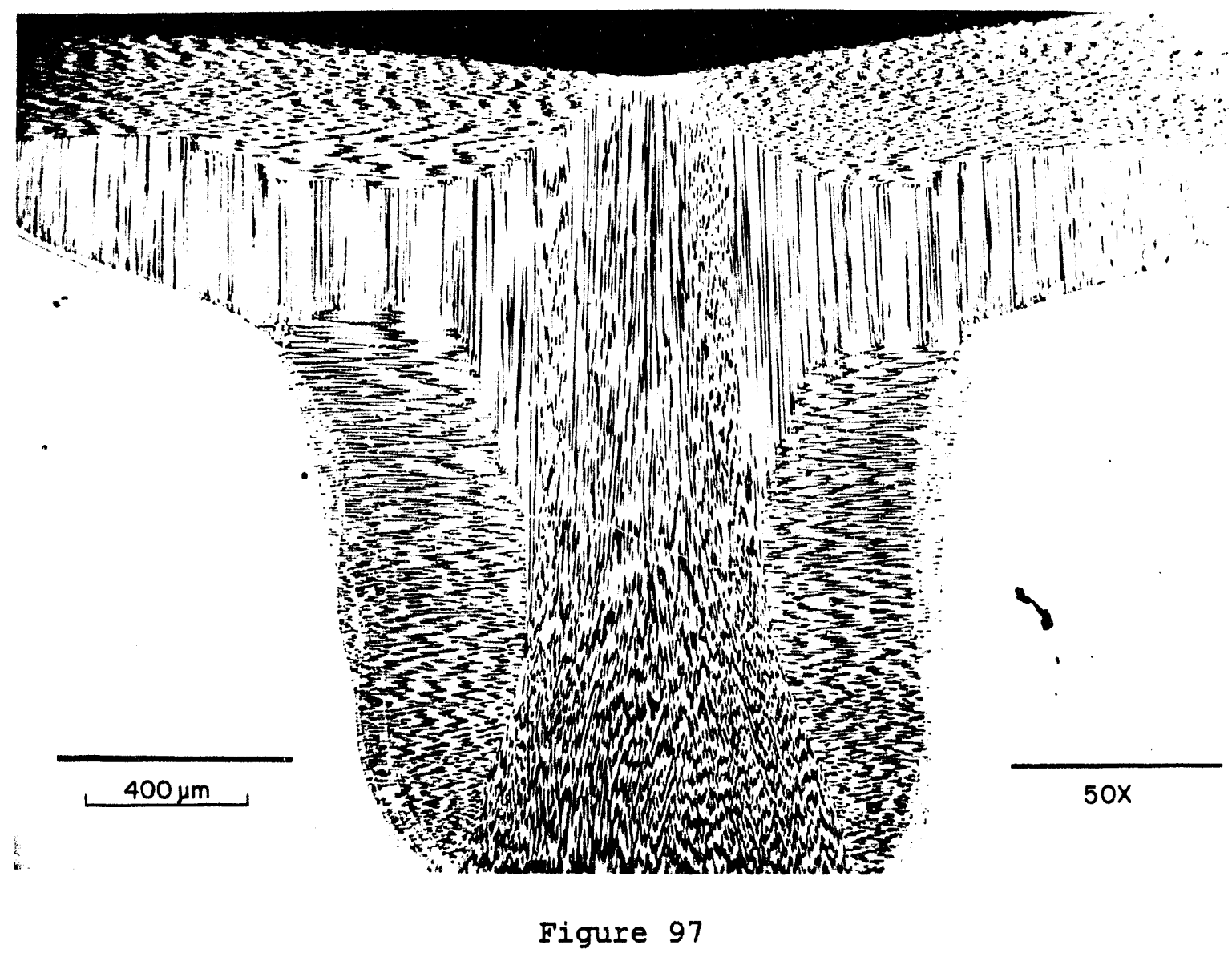

Transverse View of EB Weld $2^{\circ}$ Deviation from [100] Direction 


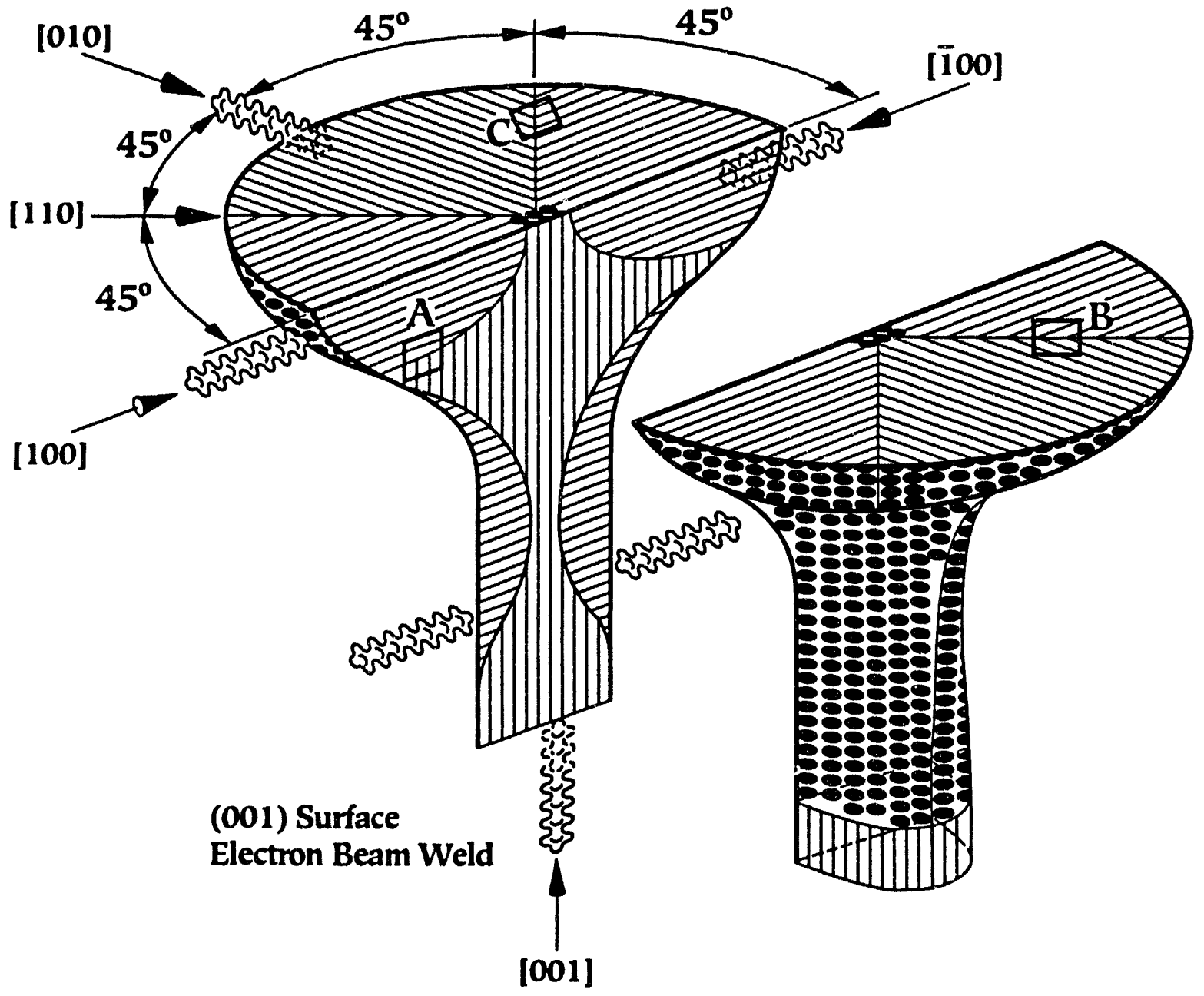

Figure 98

Three-Dimensional Reconstruction

Identifying High Magnification Notations 


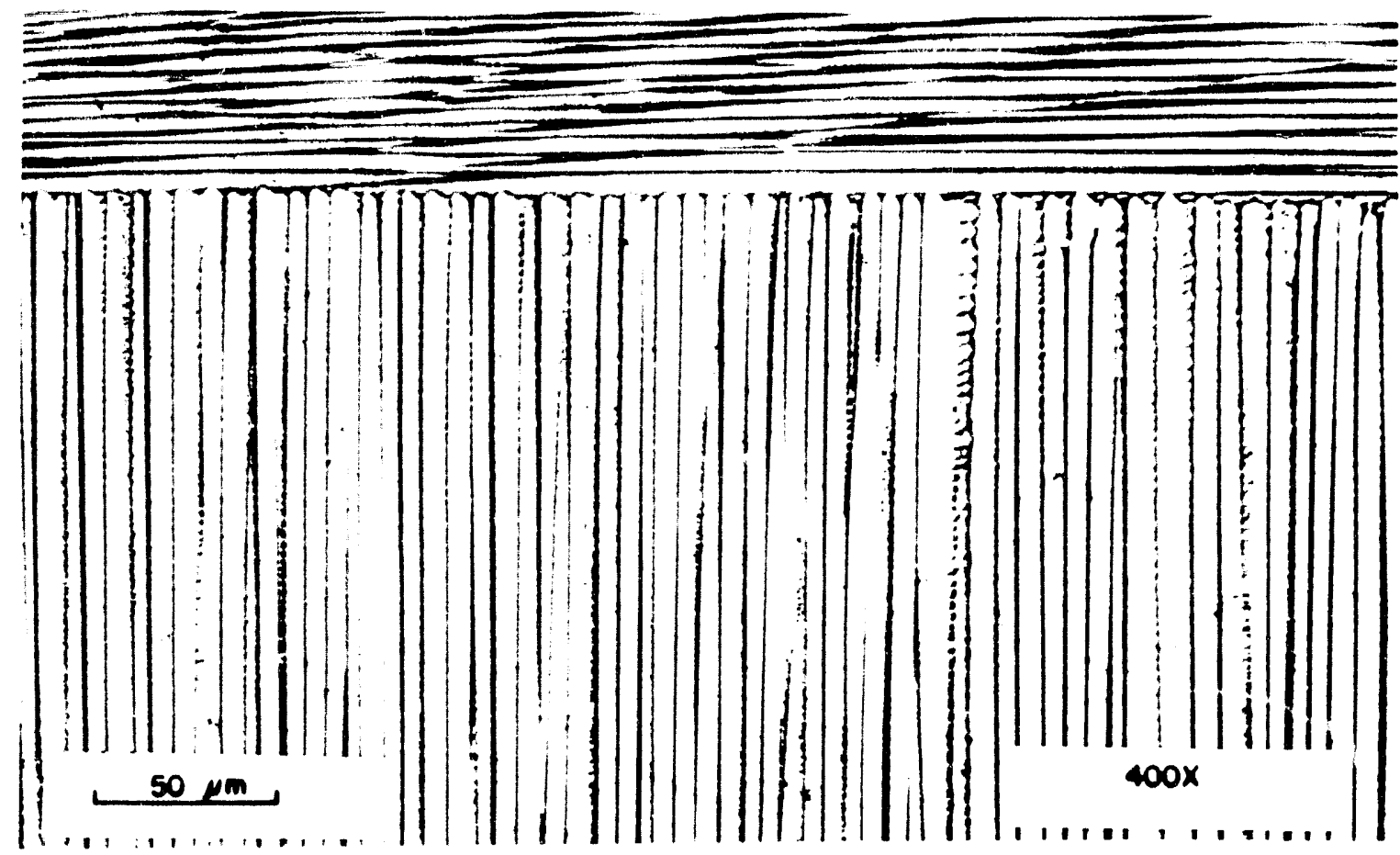

Figure 99

High Magnification of EB Weld [100] Transverge View (Area "A" of Figure 98) 
transverse view is shown in which the dendrites grew across the photomicrograph prior to those dendrites growing in the [001] direction. The [100] dendrites grew uninterrupted as compared to the intersecting [001] dendrites.

Evident in other welds were dendrites which grew at various speeds relative to each other. Area "B" of Figure 98 is microstructurally represented in Figure 100. This was shown in the top surface, in whitch the [100] and [010] dendrites met along the [110] direction, bit formed a "stair step" intersection instead of a straight line. Slight impurity levels in the single crystal resulted in a slightly slower dendrite tip speed in one direction relative to another direction [31]. This phenomena was also evident in area "C" of Figure 98 and is microstructurally seen in Figure 101, where a section of [100] dendrites grew prior to the [010] dendrites, forming a flat section of the microstructure instead of a section in which these dendrites met along the [110] direction. Another explanation of this phenomenon rests on the dependence of the dendrite-growth velocity on the angles between the solidification front and the dendrite growth direction.

Stray dendritic growth was also observed on occasion in the single crystal weld microstructure (Figure 102). This stray growth is illustrates by a section of [100] and [010] dendrites interrupted by a section of [001] dendrites. Fracture of a 


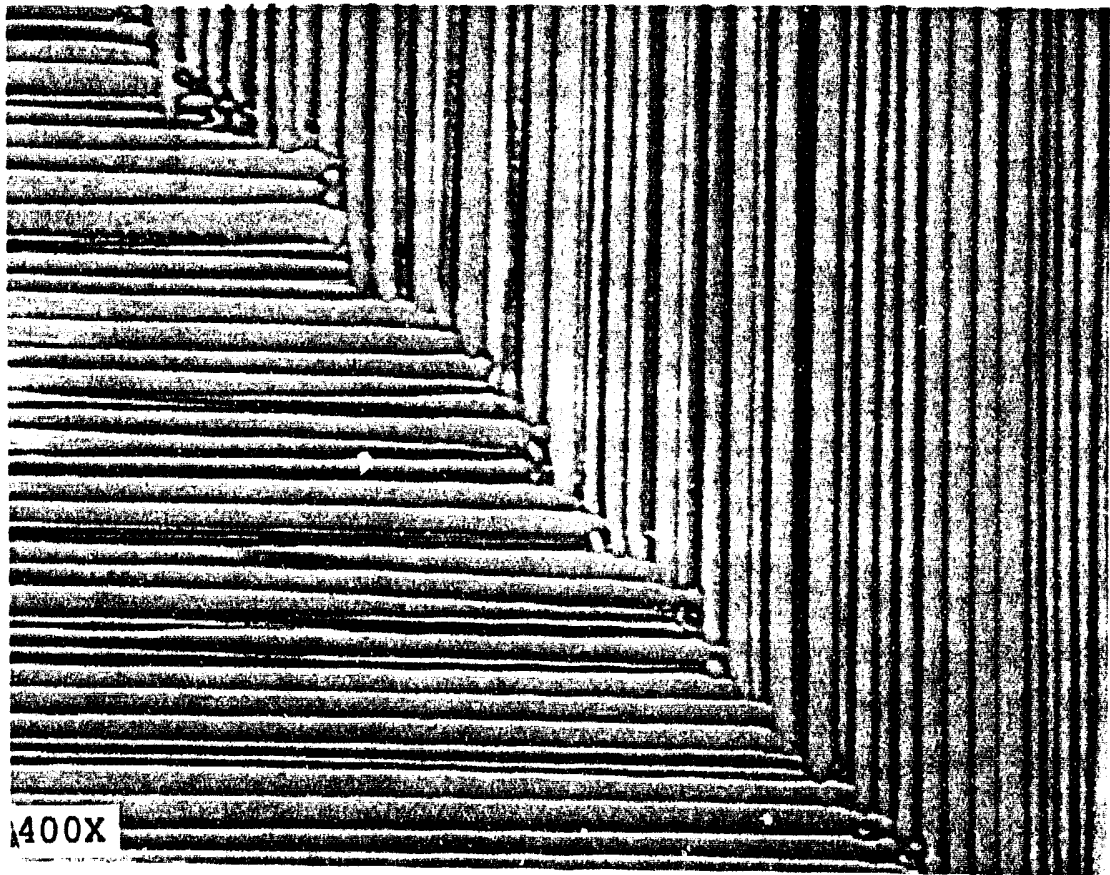

Figure 100

EB Weld (001) Surface View Along the [100] Direction

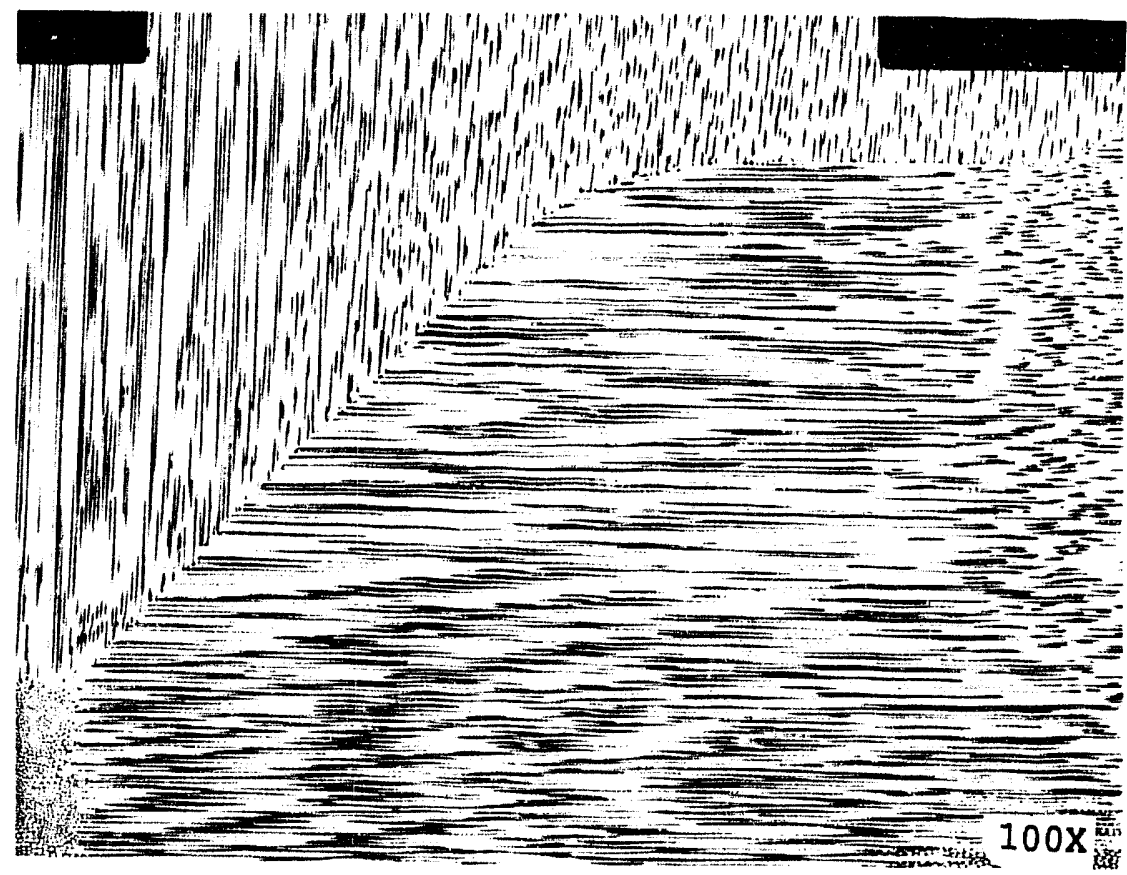

Figure 101

EB Weld (001) Surface Along the [110] Direction 


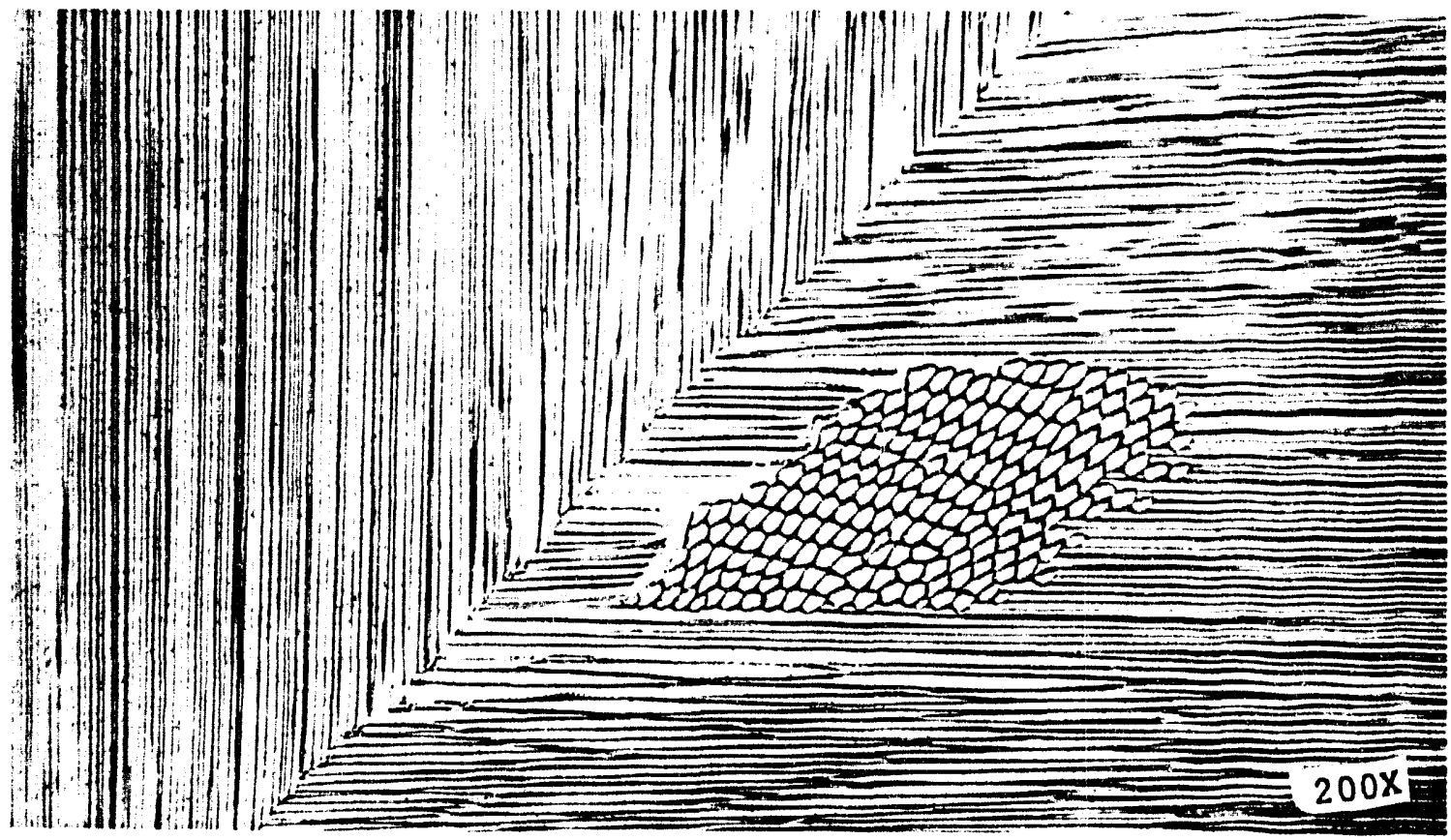

Eigure 102

EB Weld (001) Surface Stray Dendritic Growth 
dendrite tip and subsequent nucleation of the tip with a new favored growth direction. These stray growths were unfredictable and random in origin and position in the weld pool.

Final examinations of $E B$ welds were made in the form of transverse views along the [100] and [110] directions, so that metallography was performed from the edge to the center of the weld. These tests were performed to determine better the solidification and microstructural development through the welds. [110] Transverse Views: Figure 103 represents the areas in which photomacrographs are presented. For the [110] direction Figures 103b-d show the photomacrographs from the edge to the center of the weld. It could be seen that solidification occurred initially from the edge toward the center along the [100] directions, and simultaneously in the [001] direction from the bottom of the weld pool. Solidification continued in this way except for a set of dendrites growing along the [100] at the bottom of the weld pool. Finally, at the cencer of the weld, the solidification structure proceeded in the [001] direction from the bottom of the weld pool and on the ledges. Solidification continued along the sides and the top in the [100] and [010] directions meeting along the [110] direction.

[100] Transverse Views: For the [100] direction Figure 104 represents the sections in which photomacrographs were used. Similar solidification patterns could be seen in the [100] 


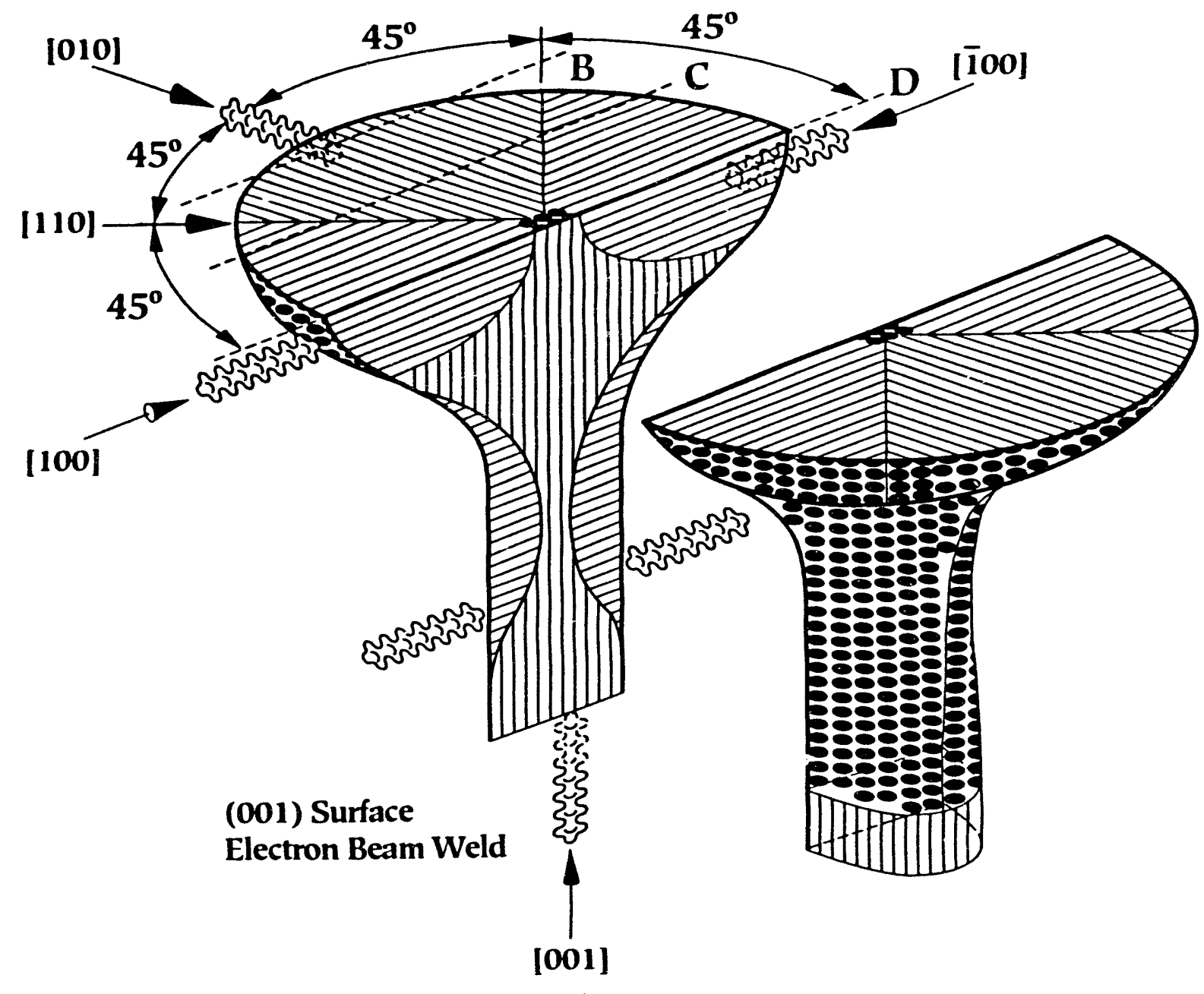

(a)

Three-Dimensional Reconstruction

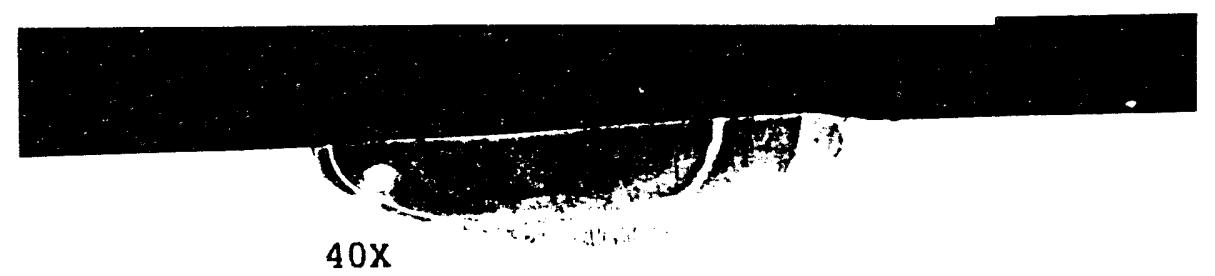

(b) 


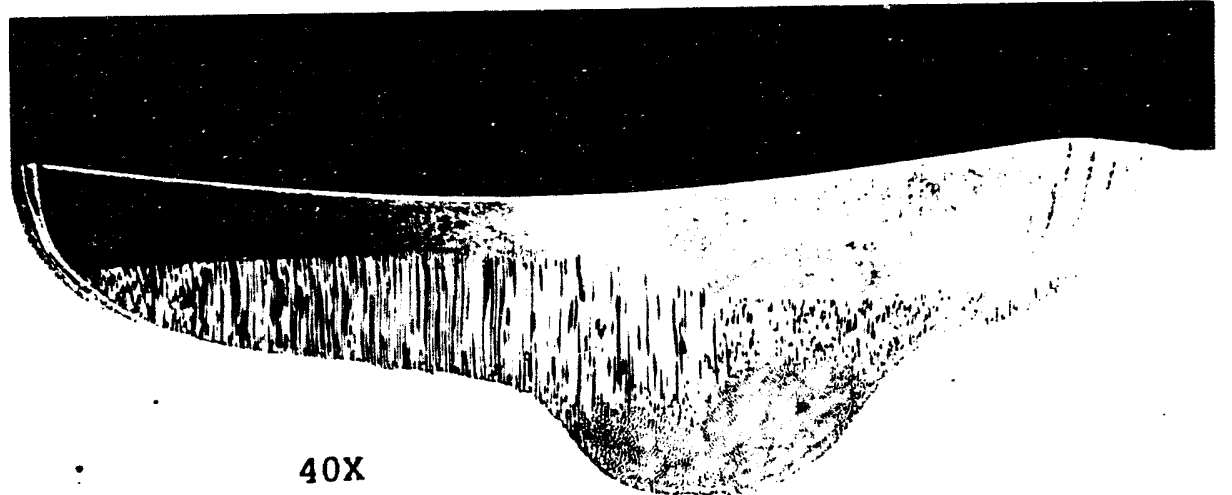

(c)

Transverse View EB Weld [110] Direction Deeper into the Weld

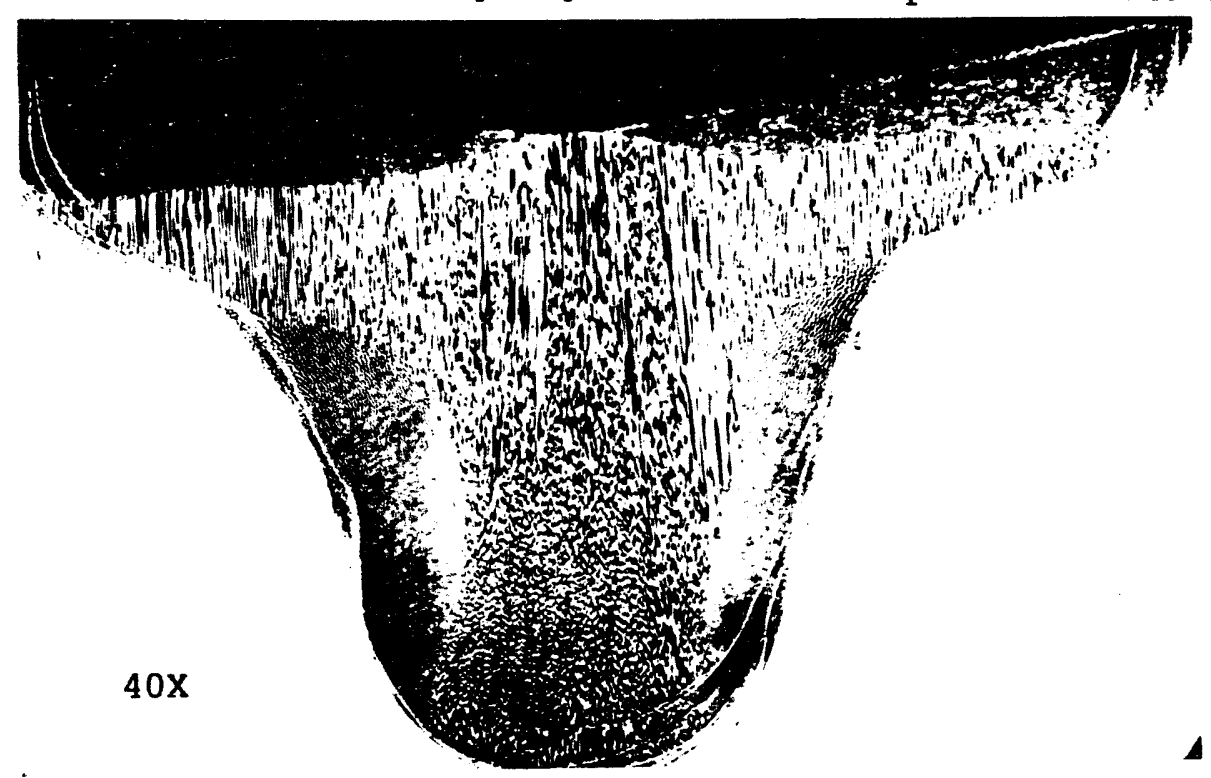

(d)

Transverse View EB Weld [110] Direction at the Center of the Weld Figure 103

Three Dimensional Reconstruction of EB Welding of (001) Plane with accompanying [110] Transverse Views 


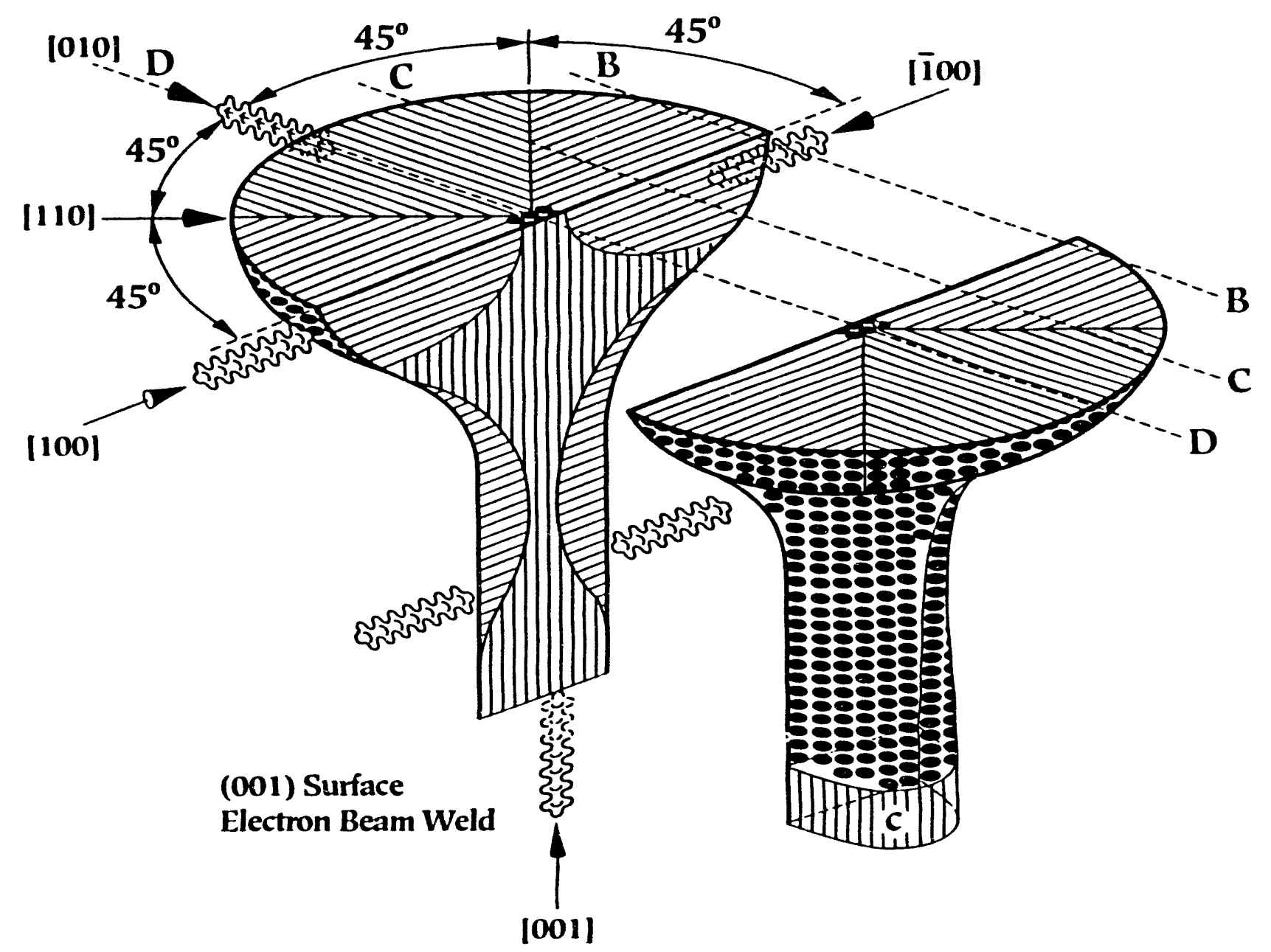

(a)

Three-Dimensional Reconstruction

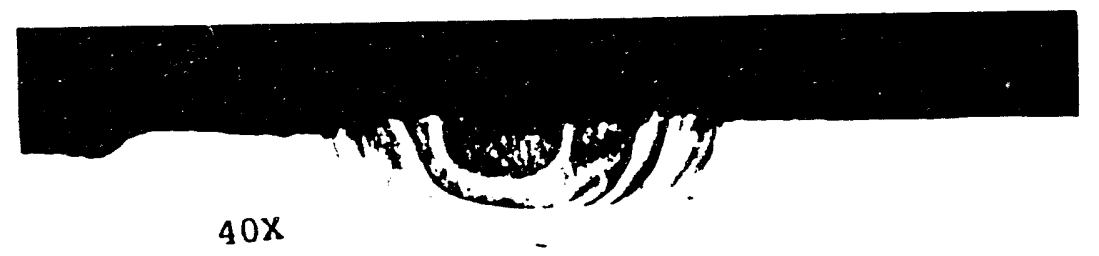

(b)

Transverse View EB Weld [100] at the Edge of the Weld 
$40 \mathrm{x}$

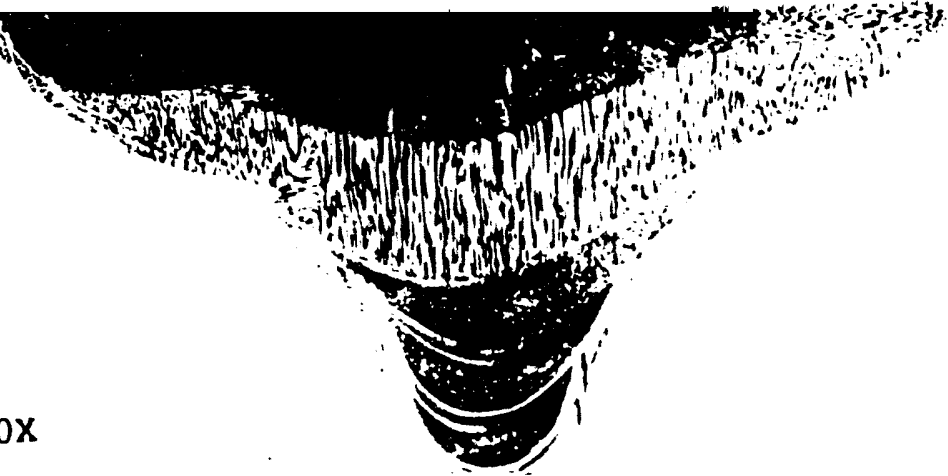

(c)

Transverse View EB Weld [100] Direction Deeper into the Weld

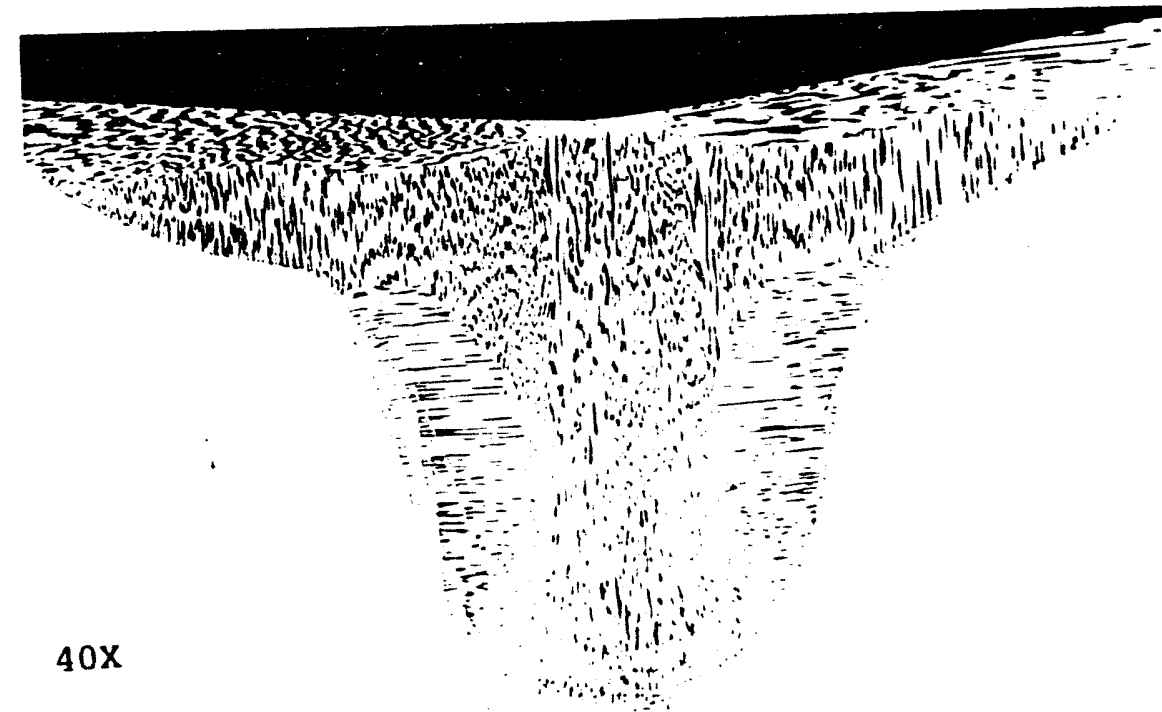

(d)

Transverse View EB Weld [100] Direction at the Center of the Weld

Figure 104

Three-Dimensional Reconstruction of EB Welding (001) Plane

with Accompanying [100] Transverse Views 
direction cut, using the same type of analysis. These results appear in Figures 104a-c. Solidification occurred along the [100] and [010] directions and simultaneously in the [001] direction.

Summary EB (001) Crystallographic Orientation

The solidification for the $\mathrm{EB}$ welds is more complex then that process in the GTA welding. This is because the variation in weld pool shape causing different favored growths in the weld. Solidification for an $\mathrm{EB}$ weld on a (001) oriented surface occurred on the surface in the [100] and [010] directions and proceeded downward into the weld. Simultaneously, solidification of the weld ledges proceeded from the base metal upward in the [001] direction toward the surface of the weld. Deeper into the weld, below these ledges, solidification proceeded as it did on the surface of the weld pool, with the [100] and [010] directions dendrites growing across and the [001] dendrites growing in the center of the weld. A section of the solidification extended from the bottom to the top of the weld, creating the center section of [001] dendrites which were visible at all depths in the top surface photomacrographs. 


\section{Comparison Between Electron Bearn and Gas Tungsten Arc Welding}

There were several major differences between the two welding methods and the weld pool shape of the spot weld produced. The EB melting produced a less-defined weld pool shape; the edges of the pool had multiple melt sites and there was not a clearly defined partially melted zone (Figure 105). This is due to the keyhole type of penetration produced by EB welding. These figures illustrate the partially melted zone for the EB and stationary GTA welding methods. In the GTA photomicrograph a line around the weld microstructure is seen that identifies the partially melted zone from the unaffected base metal. This line is not visible in the $E B$ welds because of the multiple penetration depths.

As previously discussed, the EB melting did not produce a consistent weld pool shape throughout the depth of the weld. These microstructural manifestations were accounted for by the inhomogeneities of the EB heating. However, GTA stationary welds resulted in a more defined weld-pool shape and no areas of melt and remelt (Figure 106, 107). These figures show the (001) surface and the [100] transverse views. A comparison of the weld pool shapes can be directly performed for both the surface view and the transverse view which show the more-uniform shape 

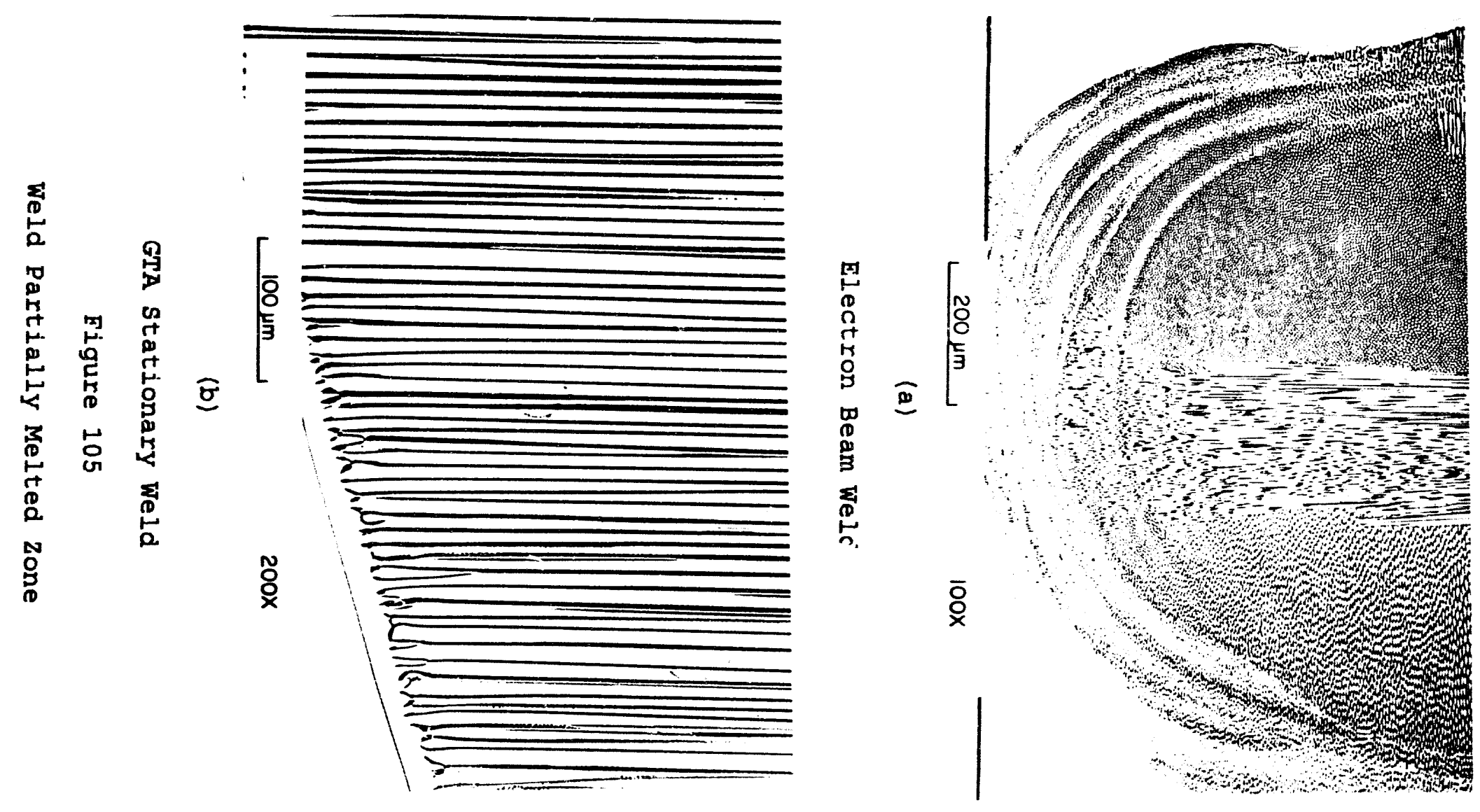


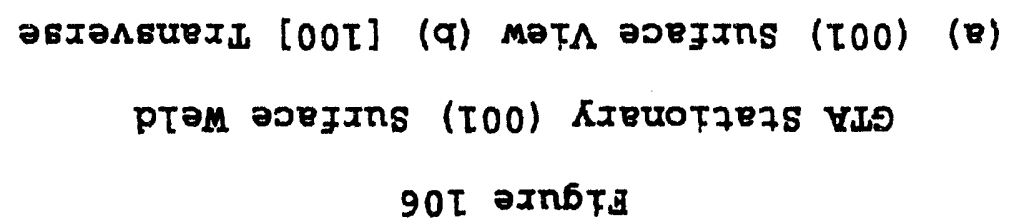

(q)

X9 I

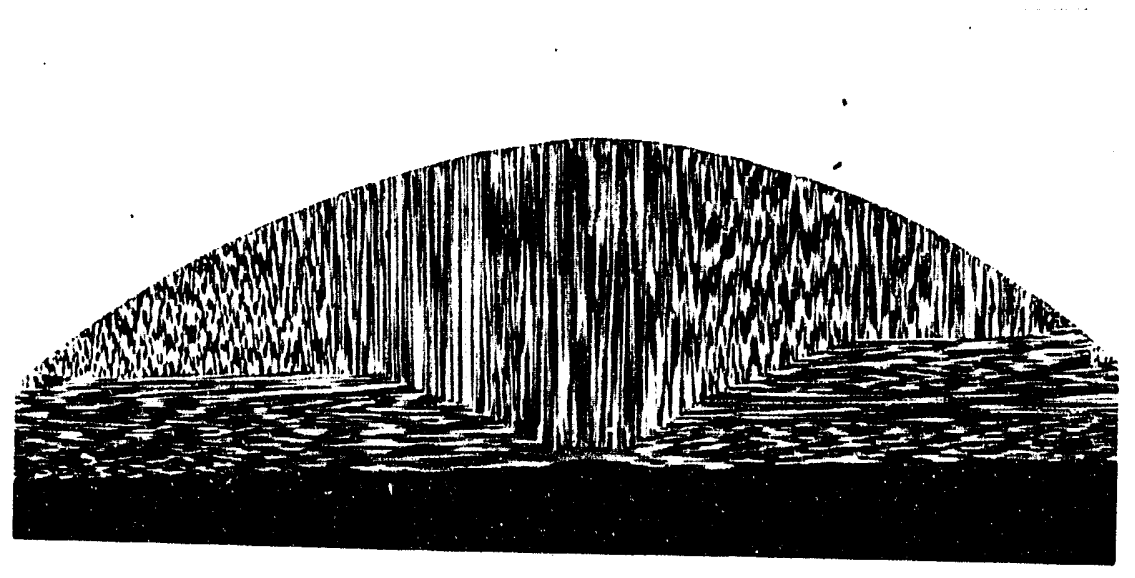

(e)

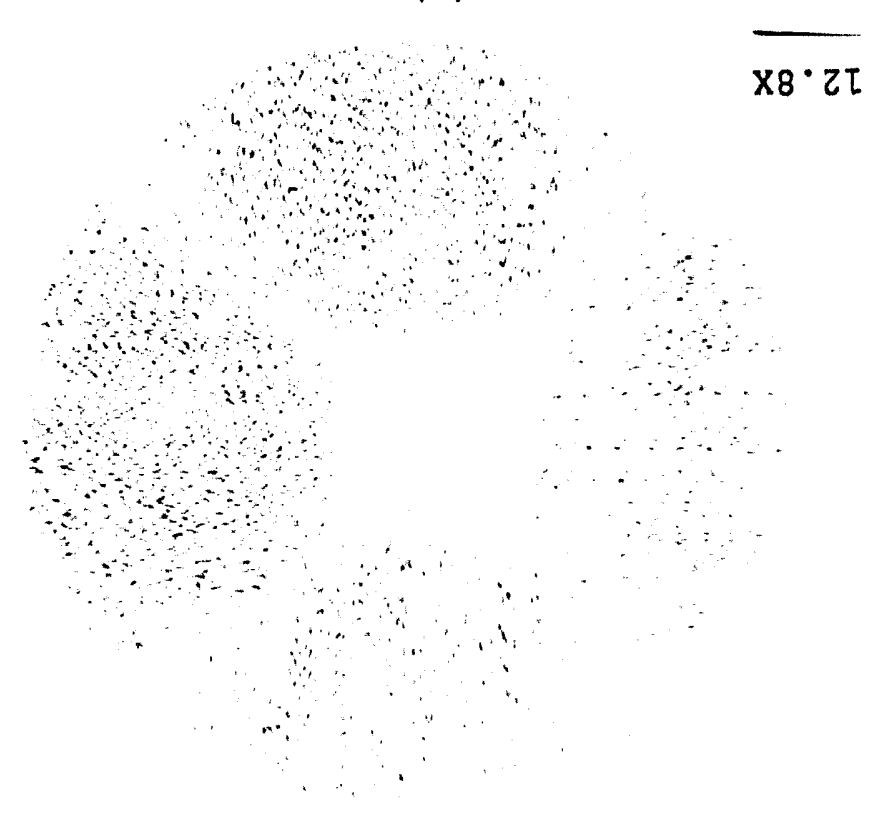



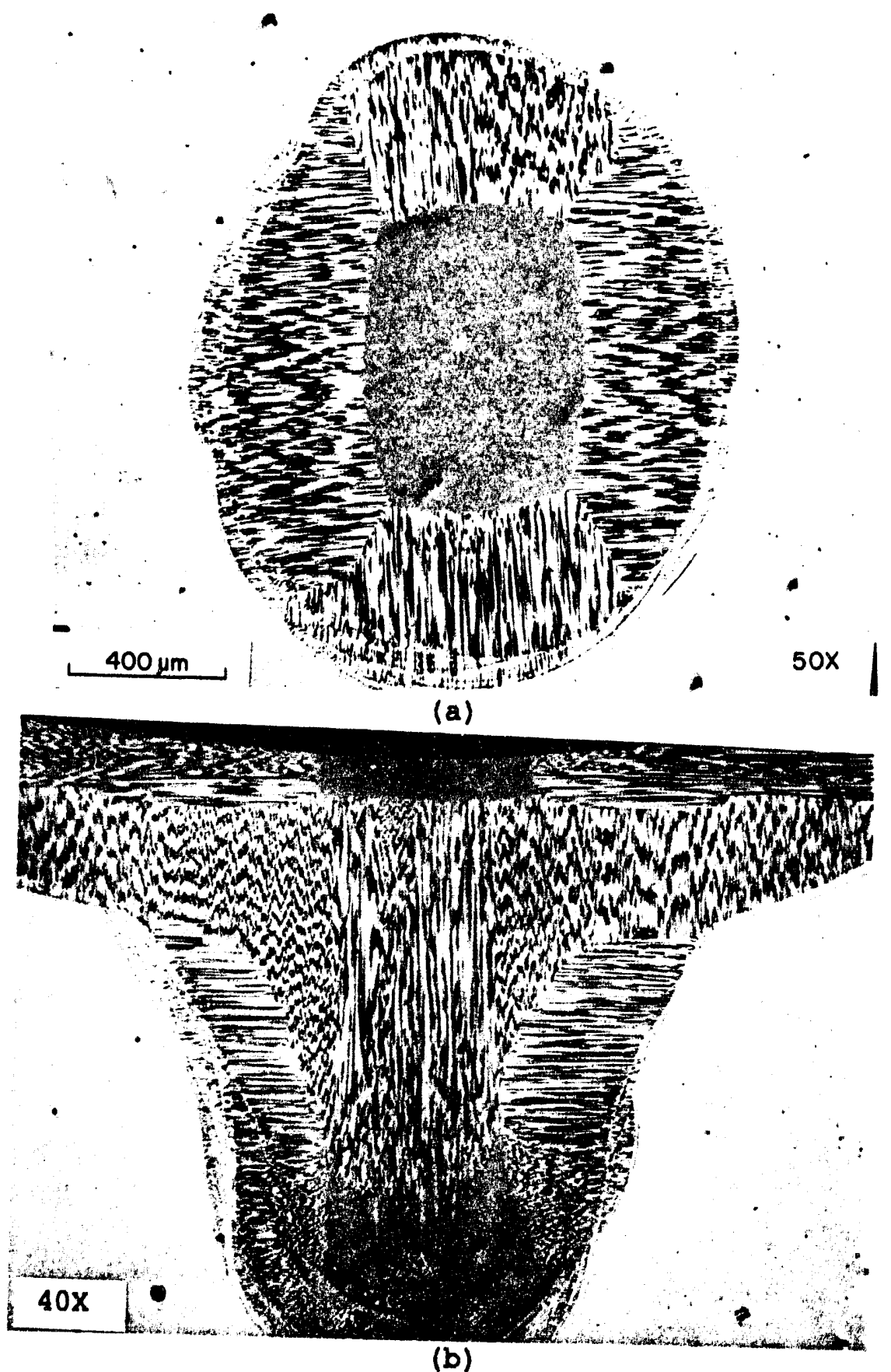

(b)

Figure 107

EB Weld (001) Surface

(a) (001) Surface View (b) [100] Transverse View 
acquired by the stationary GTA welding. There also seem to be fewer stray dendritic growths in the GTA welding process as compared to the EB welds. The resultant microstructures had similarities in growth patterns on the surface, but the changing weld pool shape in the EB heating caused large variations in the melt-pool symmetry which became evident in the details of the weld microstructure.

\section{Sumary}

This section presentz details of the solidification process in the stainless-steel single-crystal spot welds. It was assumed that solidification of the spot weld would be along the preferred growth directions of the crystal and that the weld-pool shape would influence the resultant microstructure. From the above discussion, each of these assumptions proved to be valid and were verified experimentally. This investigation resulted in the formation of three-dimensional reconstructions of the resultant microstructures created from the photomacrographs. Finally, each of the planes welded resulted in a unique microstructure as well as each welding method producing a unique microstructure. 


\section{CHAPTER 5}

\section{CONCLOSIONS}

The microstructural analysis and characterization of spot welds for the $(100),(110)$, and (111) oriented planes of stainless steel single crystal leads to the following conclusions:

1. The resultant microstructure of each plane depended on the weld pool shape and the crystallography, which determined the "easy growth" directions of the sample.

2. There were variations in the resultant microstructure for each plane and welding method.

3. The microstructure of welds made by electron beam and gas tungsten arc differed significantly because the elongated weld-pool shape of the electron-beam welds produced different solidification patterns as compared to the more-uniformly-shaped gas-tungsten-arc welds.

4. The resultant microstructures demonstrated repeatability within individual planes and welding methods.

5. The microstructure of overlapped spot welds was found to depend on the crystallography of the sample and not on the existing microstructure of the previous weld.

6. The (111) plane presented problems in obtaining a well defined circular weld pool shape. 
7. The welds on the (111) face required noticeably less heat to achieve the desired size spot weld as compared to the (100) and (110) planes because of the inherent qualities of the orientation.

8. Weld ripples, which appeared on the weld surface, varied in amplitude according to the crystallographic plane. The larger amplitude of the ripples on the (100) plane made them more evident than those on the (110) plane. The amplitudes decreased as a function of distance from the edge to the center of the weld and the frequency increased in this manner for each plane.

\section{Future Fork}

These findings indicate several avenues for future investigations :

1. A mathematical analysis should be conducted in order to obtain a model for predicting the microstructure for the weld pool shape) from the theories of solidification and dendritic growth for a stationary weld. These results should be compared to the experimental data obtained from the present investigation.

2. A more-uniform electron beam spot weld should be developed using a rotational device to attempt to 
eliminate the thermal non-uniformities in the electron beam welds.

3. The effects of known impurity elements intentionally added to the welding system on the weld pool shape and microstructure should be investigated.

4. The anomalous reduced heat demands for the (111) plane should be examined further. Additional work is needed to quantify the heat flow characteristics of this plane and to then extend this analysis to the two remaining planes for comparison. 
REFLRENCES

$$
\text { - }
$$

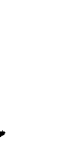

\section{REFERENCES \\ REELRENCES \\ REEMRENCES}

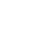

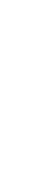


[1] Easterling, K. 1983. Introduction to the Physical Metallurgy of Welding. Butterworths \& Co. London.

[2] David, S. A., J. M. Vitek, M. Rappaz, and I. A. Boatner. 1990. "M1crostructure of Stainless steel Single-Crystal Electron Beam Welds." Metallurgical Transactions A. 21A (June): pp.1753-1766.

[3] Rappaz, M., S. A. David, J. M. Vitek, and L. A. Boatner. 1989. "Development of Microstructures in Fe-15Ni-15Cr Single Crystal Electron Beam Welds." Metallurgical Transactions A. 20A (June): pp.1125-1138.

[4] David S. A., and I. A. Boatner. 1991."Single Crystals for Welding Research." Oak Ridge National Laboratory Review.No.2: pp.33-41.

[5] Rappaz, M., S. A. David, J. M. Vitek, and I. A. Boatner. 1990. "Analysis of Solidification Microstructures in $\mathrm{Fe}-\mathrm{Ni}-$ Cr Single-Crystal Welds." Metallurgical Transactions A. 21A (June): pp.1767-1782.

[6] Kearns, W. H., ed. 1976. Welding Handbook Seventh Edition, Volume 2 Welding processes. American Welding Society. Miami, Fl.

[7] Hearns, W. H., ed. 1976. Welding Handbook Seventh Edition, Volume 3 Resistance and Solid-State Welding and other Joining Processes. American Welding Soclety. Miami, FI.

[8] Savage, W. F. and A. H. Aronson. 1966." Preferred Orientation in the Weld Fusion Zone." Welding Journal. $45(2)$ : $p p .85 s-89 \mathrm{~s}$.

[9] Zapffe, Carl A. Isis. Stainless Steels. Miami, Fl: American Welding Society.

[10] Vitek, J. M., A. Dasgupta, and S. A. David 1983. "Microstructural Modification of Austenitic Stainless Steels by Rapid Solidiflcation." Metallurgical Transactions A. 14A(9): pp.1833-1841.

[11] Zacharia, T., S. A. David, J. M. Vitek, and T. Debroy. 1989. "Weld Pool Development during GTA and Laser Beam Welding of Type 304 Stainless Steel, Part 1-Theoretical Analysis." Welding Journal. 72(12): pp.499s-509s. 
[12] David, S. A. and J. M. Vitek. 1993. "Principles of weld Solidification and Microstructures." International Trends in Welding Science and Technology, International Conference Proceedings June 1-5, 1992. Metal Parks, Ohio. ASM International: pp. 147-156.

[13] Zacharia, T., S. A. David, J. M. Vitek, and T. Debroy. 1989. "Weld Pool Development during GTA and Laser Beam Welding of Type 304 Stainless steel, Part 2-Experimental Correlation." Welding Journal. 72(12): pp.510s-519s.

[14] David, S. A. and J. M. Vitek. "Weld Pool Solidification and Microstructures." The Metal Science of Joining Metal Parks, Oh10: pp.1-9.

[15] Matsuda, Fukuhisa, Tatsuya Hashimoto, and Tomio Senda. 1969. "Fundamental Investigations on Solidification Structure in Weld Metal." Transactions of National Research Institute for Metals. $11(1):$ pp.43-58.

[16] David, S. A., and J. M. Vitek. 1989."Correlation between solidification parameters and weld microstructure." International Materials Reviews. 34(5): pp.213-245.

[17] Nakagawa, Hiroji, Mitsuaki Katoh, Fukuhisa Matsuda, and Tomio Senda. 1970. "Fundamental Solidification Mechanisms and Microstructures in GTA Spot Welds of Aluminum Sheets." Transactions of Japan Welding society. 1(2): pp. 28-39.

[18] Rappaz, M., S. A. David, J. M. Vitek, and I. A. Boatner. 'Analysis of Microstructural Development in Single Crystal Welds." Recent Trends in Welding Science and Technology. ASM International. Metal Parks, Oh10. pp. 147-151.

[19] Bobadilla, J. Lacaza, and G. Lescult.1988.Journal of Crystal Growth. 89: pp.531-544.

[20] Vitek, J. M., S. A. David, I. A. Boatner, and M. Rappaz. 1993. "Grain Competition in Blcrystal Welds." International Trends in Welding Science and Technology International Conference Proceedings. pp.167-172.

[21] Savage, W. F., C. D. Lundin, and T. F. Chase. 1968. "Solidification of Fusion Welds in Face-Centered Cubic Metals." Welding Journal. 47(11): pp.522s-526s. 
[22] Nakagawa, Hiroj1, Mitsuaki Kato, Fukuhisa Matsuda, and Tomio senda. 1970."X-Ray Investigation on Solidification Structures in Weld Metal." Transactions of Japan Welding Society. 1(1): pp.1-10.

[23] Nakagawa, Hiroj1, Mitsuaki Kato, Fukuhisa Matsuda, and Tomio Senda. 1973. "Features in Weld Solidification of High Purity Aluminum Sheets." Transactions of Japan Welding Society. 4(1): pp.43-48.

[24] Kato, Mitsuaki, Fukuhisa Matsuda, and Tomio Senda. 1972. "Growth Features of Subgrains and Columnar Cryotals in Aluminum Weld Metal." Transactions of Japan Welding Society. 3(1): pp.59--68.

[25] Nakagawa, Hiroji, Mitsuaki Katoh, Fukuhisa Matsuda, and Tomio Senda. 1971. "Crystallographic Investigation for origination on New Columar Crystal in Aluminum Weld Metal Using Single Crystal Sheet." Transactions of Japan Welding Society. 2(1): pp.1-9.

[26] Nakagawa, Hiroji, Mitsuaki Katoh, Fukuhisa Matsuda, and Tomio Senda. 1971. "Crystallographic Anisotropy of Columnar zone in Aluminum Weld Metal." Transactions of Japan Welding Soclety. 2(1): pp.10-20.

[27] Kou, S. and Y. Le. 1986. "Nucleation Mechanisms and Grain Refining of Weld Metal." Welding Journal. 65(12): pp.305s$313 \mathrm{~s}$.

[28] Passoja, D. and R. Bakish. 1970."Solidification Structure of EBW Single Crystals. "Electron and Ion Beam Science and Technology 4th International Conference. Eletrothermics and Metallurgy Division, The Electrochemical Society. New York, NY. pp. 213-231.

[29] Copley, S. M., D. Beck, O. Esquivel, and M. Bass. 1978. "Laser Melt Quenching and Alloying. "Laser, Solid Interaction and Laser processing-Conference Proceedings No. 50. New York. American Institute of Physics: pp. 161172 .

[30] Anthony, T. R. and H. E. Cline. 1977."Surface rippling induced by surface-tension gradients during laser surface melting and alloying." Journal of Applied Physlas. 48(9): pp. 3888-3894. 
[31] Esquivel, O., J. Mazumder, M. Bass, and S. Copley. 1980."Shape and Surface Relief of Continuous Laser-Melted Trails in Udimet 700." Rapid Solidification Processing, principles and Technologies II. Claitor's Publishing, Baton Rouge, LA: pp.

[32] Brice, J. C. The Growth of Crystals from the Melt. North Holland Publishing, Amsterdam. pp. 135-155.

[33] Ogle, J. C. and R. E. Reed. Private Communication.

[34] Peavler, R. J. and J. I. Lenusky. Angles Between Planes in Cubic Crystals IMD Special report Series No. 8. New York. The Metallugical Society.

[35] Surface Texture Parameters. Taylor-Hobson Manual Talsurf 10 .

[36] Parr, J. Gordon and Albert Hanson. 1965. An Introduction to Stainless Steel. Metal Parks, Ohio. American Society for Metals.

[37] Reed-Hi11, Robert E. 1973. Physical Metallurgy Principles Second edition. New York: D. Van Nostrand Company. 
1 


\section{Appendix A \\ Initial helding studies}

To conserve the limited supply of single crystals, polycrystalline samples of type 308 stainless steel were used for a variety of basic $\mathrm{EB}$ welding experiments.

\section{Polyorystalline}

\section{Welding Parameters}

The samples were $2.54 \mathrm{~cm}$ square with a thickness of $0.32 \mathrm{~cm}$ and were used to obtain initial $\mathrm{EB}$ welding parameters in order to obtain a 80-908 penetration in the single crystals. These were tests made by adjusting the beam and focal currents on the EB welder.

\section{Weld Pool symmetry}

Tests verifying the symmetry in the weld pool were performed because uneven heat conduction was possible in welds placed near the edge of a sample. Welds positioned in two corners, the center, and center of the bottom were compared (Figures 108a-c). Weld-pool shapes for welds approximately $0.50 \mathrm{~cm}$ from the edge of the sample showed no significant deviations in the weld-pool shapes. Similar types of testing were performed with GTA welding 


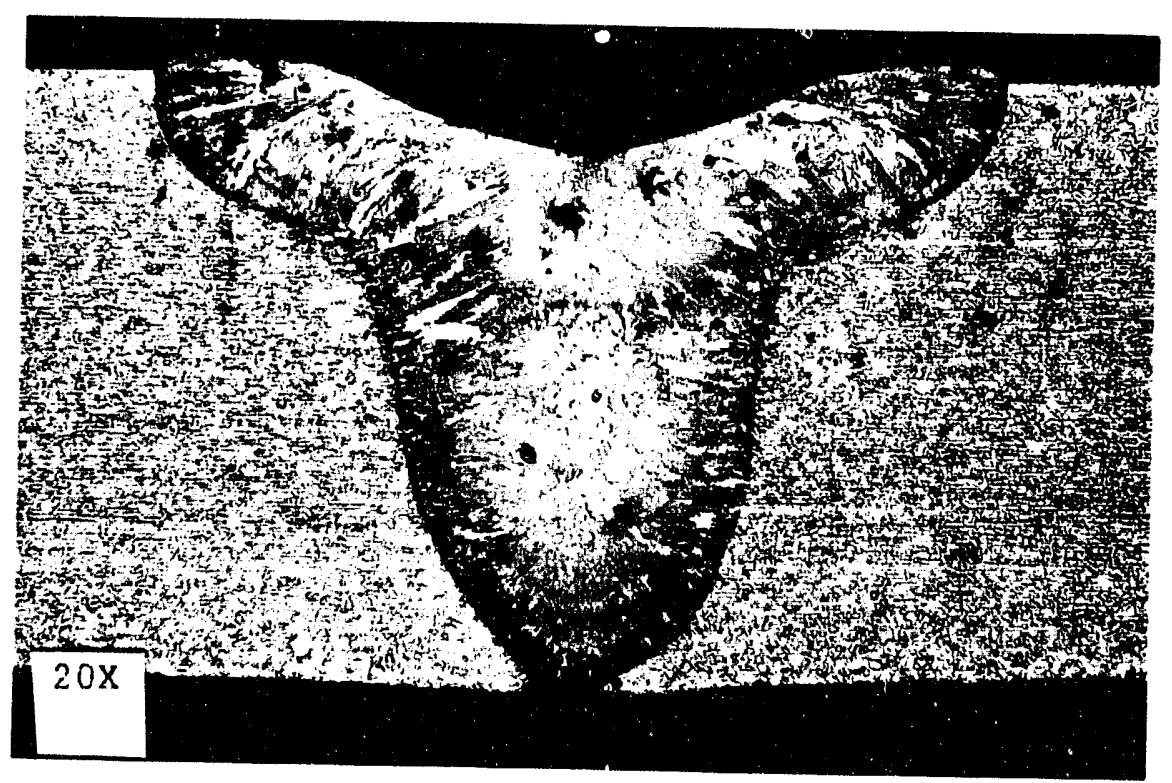

(a)

Polycryotal Weld Placed in the Upper Right Corner of Sample

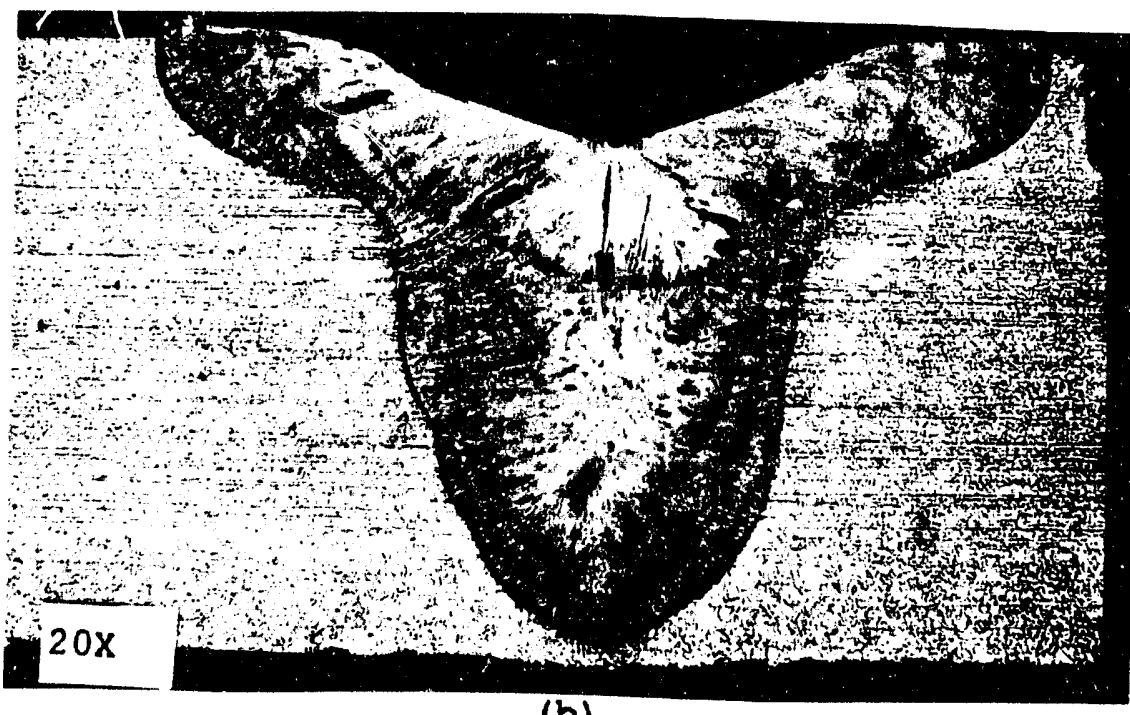

(b)

Upper Left Corner of Sample

Figure 108

Polycrystal Weld Placed in Various Positions 


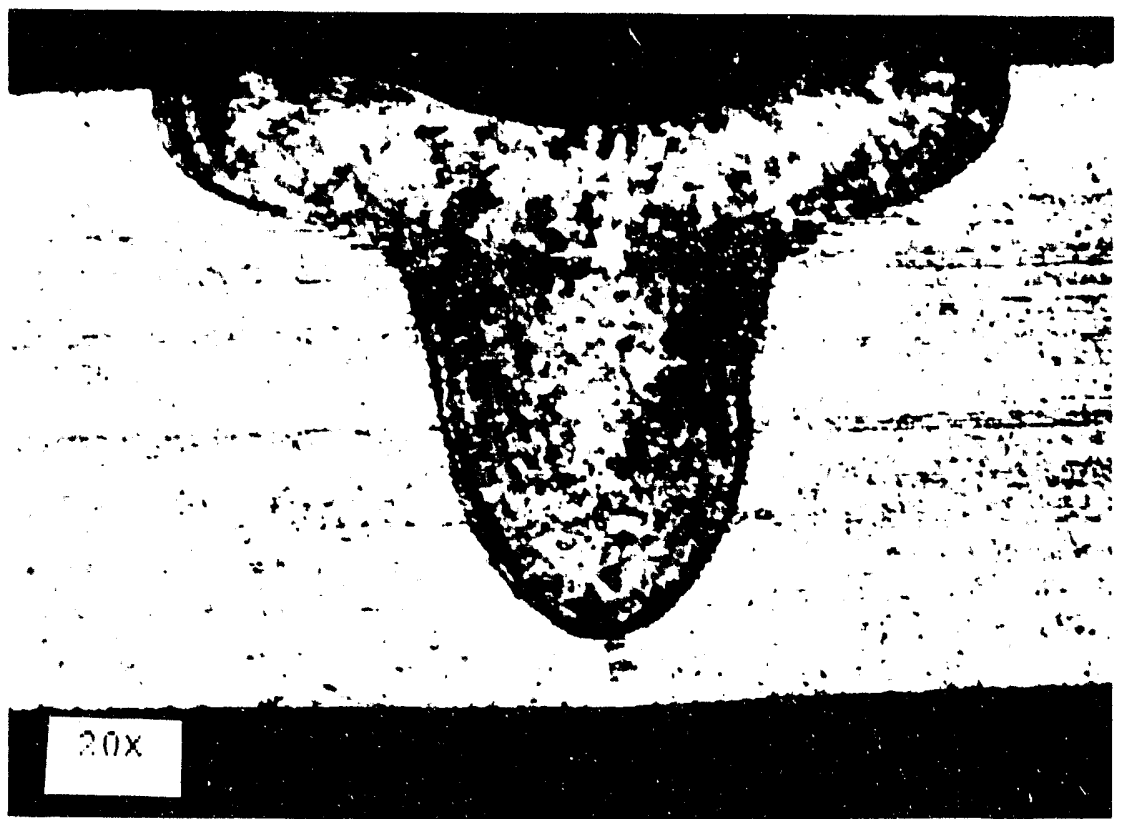

(c)

$$
\begin{aligned}
& \text { Bottom Center of Sample } \\
& \text { Figure } 108 \text { Continued }
\end{aligned}
$$

using stationary and rotational methods to determine the parameters which would achieve the desired 80-908 penetration.

\section{single Crystal}

Welding studies on single crystals determined the effects of extended welding times and multiple welds per crystal on the weld pool shape. 
Weld Time studies

The first set of time studies was made using a range of welding times on various single crystals (Table 4). From this work it was seen that the penetration increased as a function of increased welding time.

Table 4

Variations in Penetration With Increased Welding Time

\begin{tabular}{|ccc|}
\hline Sample & $\begin{array}{c}\text { Time } \\
\text { (sec) }\end{array}$ & $\begin{array}{c}\text { Penetration } \\
\text { [cm] }\end{array}$ \\
\hline JSTg & 1.0 & 0.1829 \\
JSTg" & 1.5 & 0.2057 \\
JST10 & 2.5 & 0.2108 \\
JS1.18 & 2.6 & 0.2379 \\
JS2.18 & 2.6 & 0.2106 \\
JS $4.1 \mathrm{~A}$ & 2.6 & 0.2273 \\
JS5-1A & 2.6 & 0.2159 \\
"Second wold on that perticular crystal \\
\hline
\end{tabular}


Multiple spot Welds Per Crystal

Multiple-spot-weld experiments showed that the second weld on a crystal developed a significant increase in penetration compared to the first weld. Residual heat from the first weld caused this effect. These tests established a waiting time between welds to decrease the phenomenon. Figure 109 shows a comparison of the penetration from the first to the second weld on a crystal. 


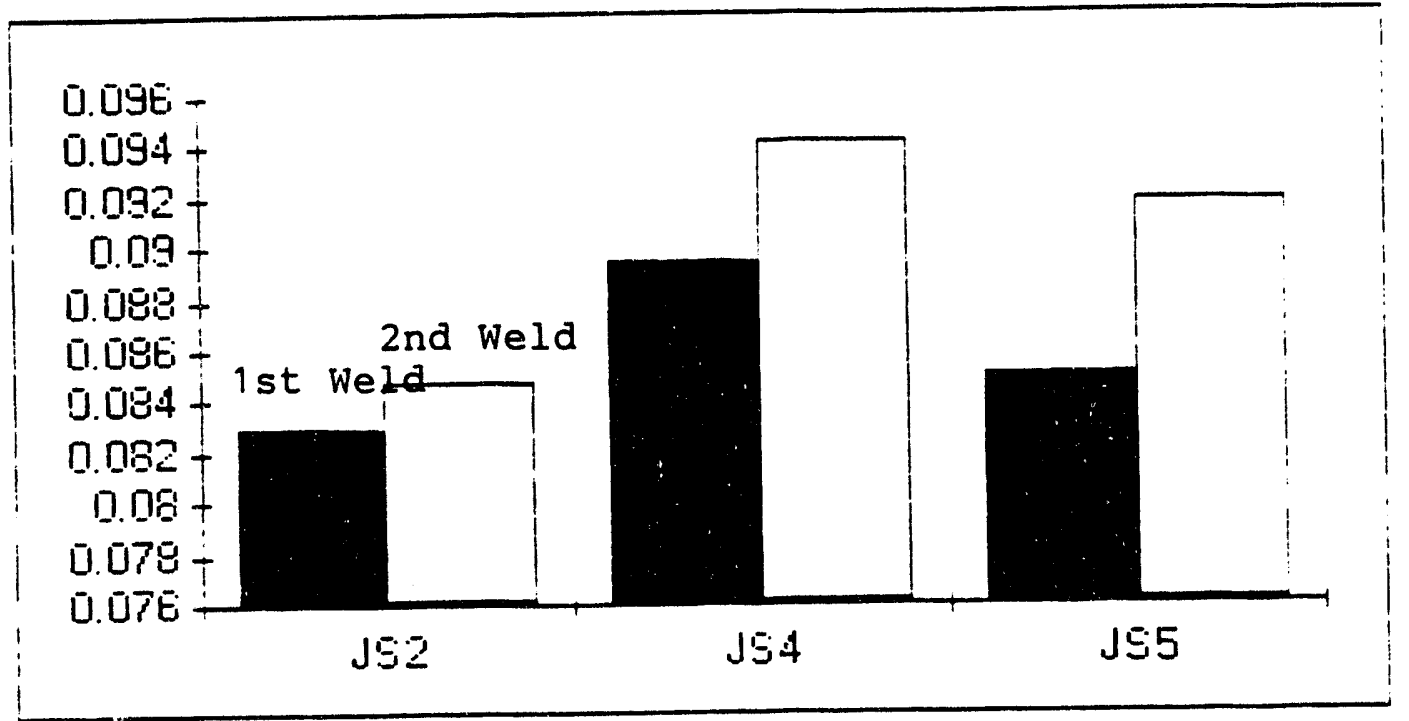

\begin{tabular}{|c|c|c|}
\hline Samale & $\begin{array}{l}\text { Time } \\
\text { Tsen] }\end{array}$ & $\begin{array}{l}\text { Perietr } \\
\text { [iri] }\end{array}$ \\
\hline . & 2.6 & 0.10847 \\
\hline $192-1 E$ & 26 & 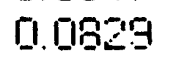 \\
\hline .154-1ش & $2 . E$ & ПП ПВЯЕ \\
\hline $.154-1 E^{*}$ & $2 E$ & 0.01742 \\
\hline $.1 S E_{1-1.4}$ & 2.6 & ח ח \\
\hline $15 F_{1}-1 B^{-:}$ & 2. E & ㅁ. ก.919 \\
\hline
\end{tabular}

Note: For the 2. E sec the values were averaged

* Secound weld or that partioldar orystal

Figure 109

Comparison of Weld Penetration For Multiple Welds Per Crystal 


\section{Appendix B}

\section{Heat sink study}

This appendix reports a study of the effects of the clamps on the weld pool shape for $E B$ welding. There was concern that the method of fixing the sample to the copper plates was influencing the heat flow to produce the oblong shapes observed in the $E B$ welds. To see if the clamps caused this effect two welds were placed symmetrically on a crystal. One weld was clamped as in the normal procedure and one weld was unclamped (Figure 110). The welding system was set up to produce a nonpenetration weld on the unclamped weld (Figure 111a-c). This test proved that the clamps had no effect on the oblong shape (Figure $112 a-b)$. However, there was a substantial effect on the heat input to the crystal. The unclamped weld did not transfer heat to the copper plates as efflciently. The results showed that the overall diameter of the clamped weld was much smaller. Heat extraction by the copper base and clamps and the excellent contact between the copper and the crystal of the clamped case caused this effect. Without the clamps, the contact was not as efficient and lessened the heat transfer. The microstructures of the welds were identical in each case of the EB welds. This microstructure has been described in detall in the (001) EB welding section. 

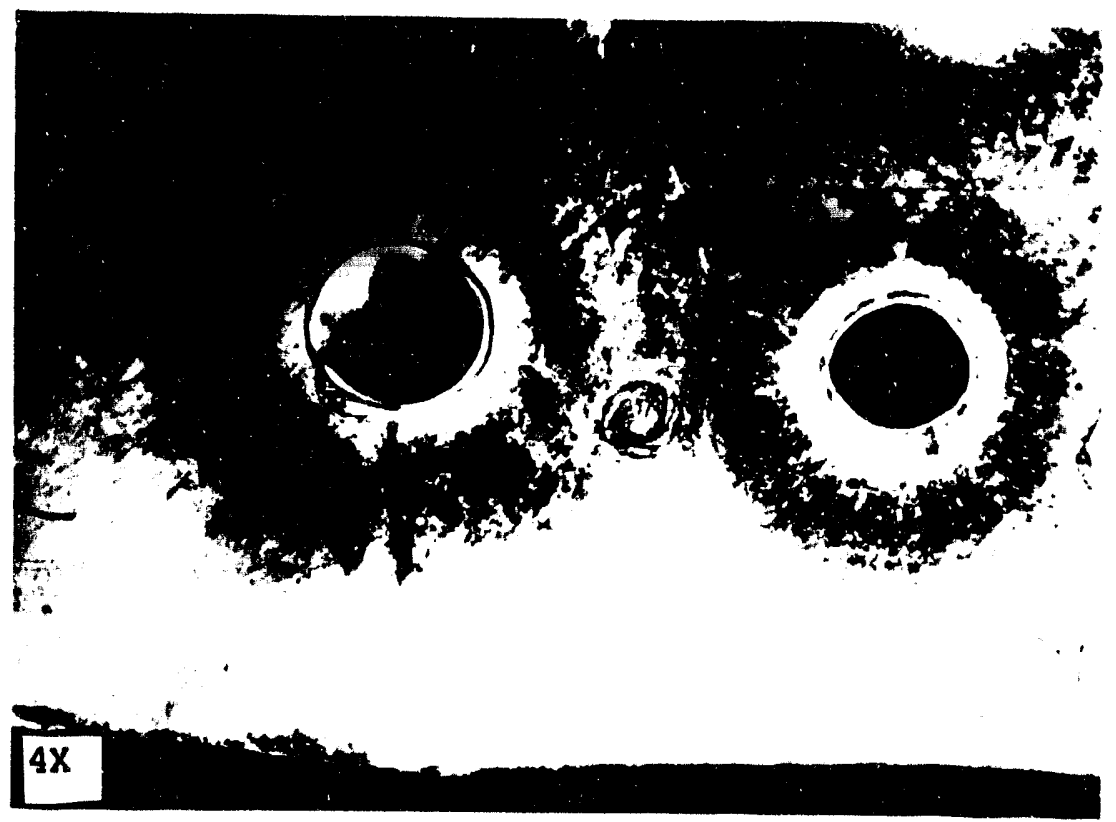

Figure 110

(001) Top Surface View of Clamped and Unclamped EB Weld

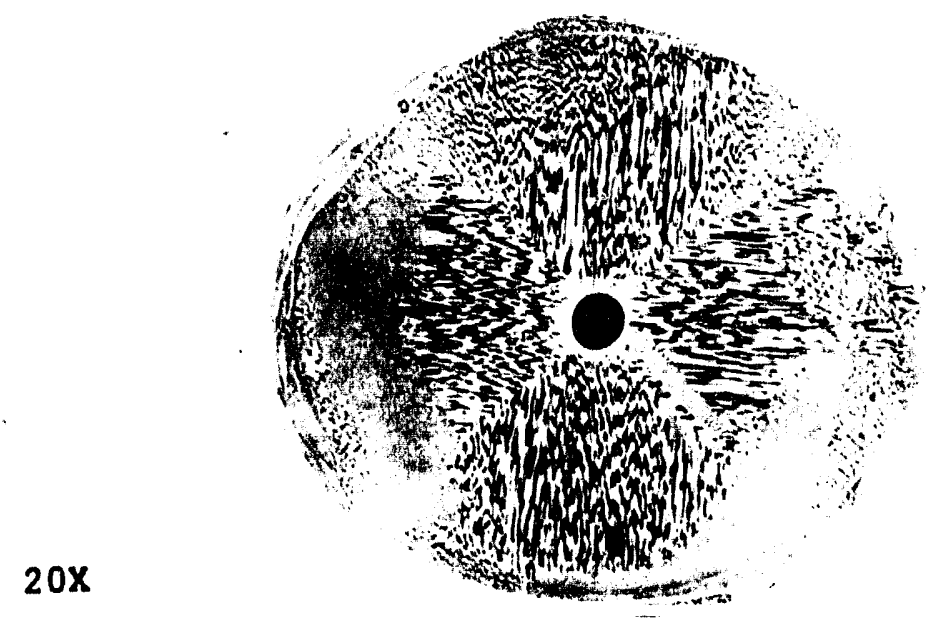

(a)

$$
\text { iop surface }
$$

Figure 111

(001) Unclamped EB Welds Surface Views 


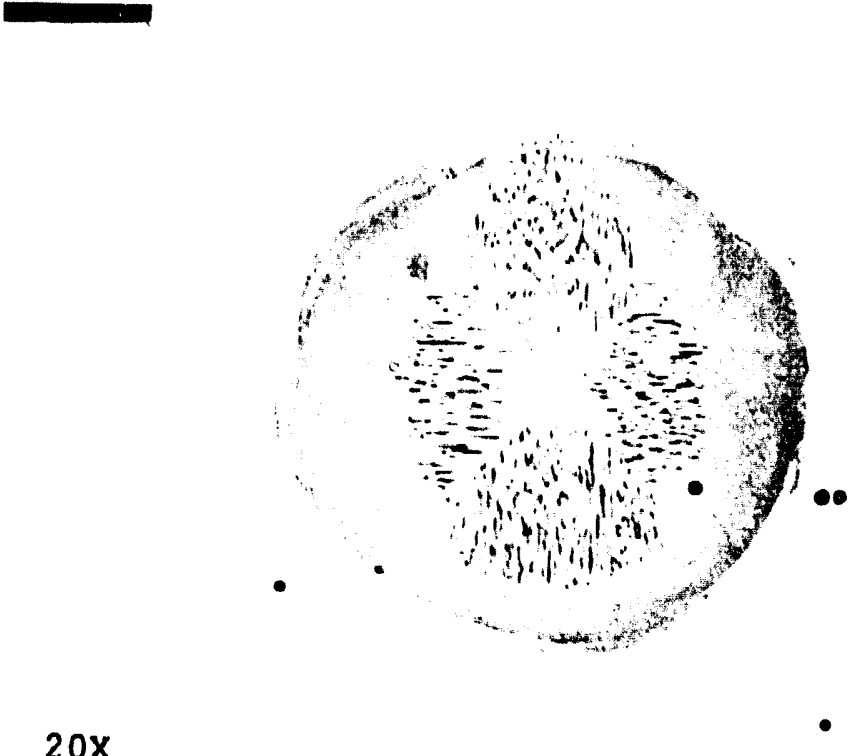

(b)

(001) Unclamped Top Surface at Depth EB Weld

$20 x$

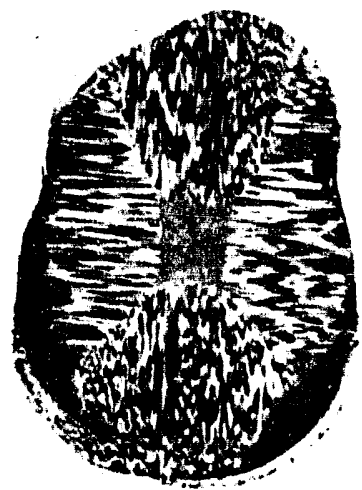

(c)

(001) Unclamped Top Surface at Oblong Shape

Figure 111 Continued 


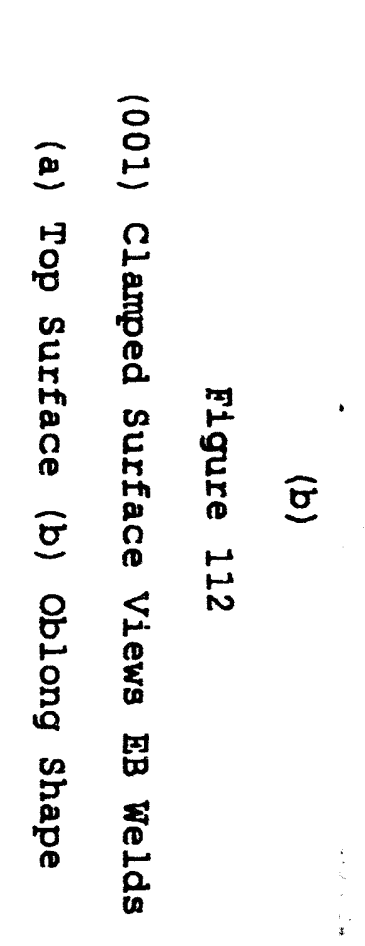

$\stackrel{N}{x}$ 


\section{Appendix C \\ Tri-Aro Welding study}

To produce a well-defined circular weld pool one additional welding method was attempted, which proved to be unsuccessful. This method used a Tri-Arc Welder (Figure 113) to produce a rotational tungsten weld in an atmosphere of 1008 argon on the (011) plane. The welding parameters are shown in Table 4 . The resultant weld pool shapes were not symmetrical and were inconsistent in their formation (Figure 114a-d). The "dimplen in the center of the weld was substantially off-center and resulted in very non-symmetric transverse-view microstructures. The microstructure of these welds was simslar to that obtained for the stationary and rotational GTA welds for the (011) plane. There was also an increase in stray dendritic growth for this method, as seen at the top of the transverse view (Figure $114 \mathrm{~b}, \mathrm{c})$. The top surface of the Tri-Arc welds had better symmetry as compared to the EB welds, but the excellent symmetry of the GTA welds was not evident in the Tri-Arc welds. There were several reasons for the inconsistency encountered in this method. First, the method employed a hand-held electrode which allowed for movement of the electrode during welding. Second, the electrode also had an arc gap that was not fixed which 
Table 5

Tri-Arc Welding Parameters

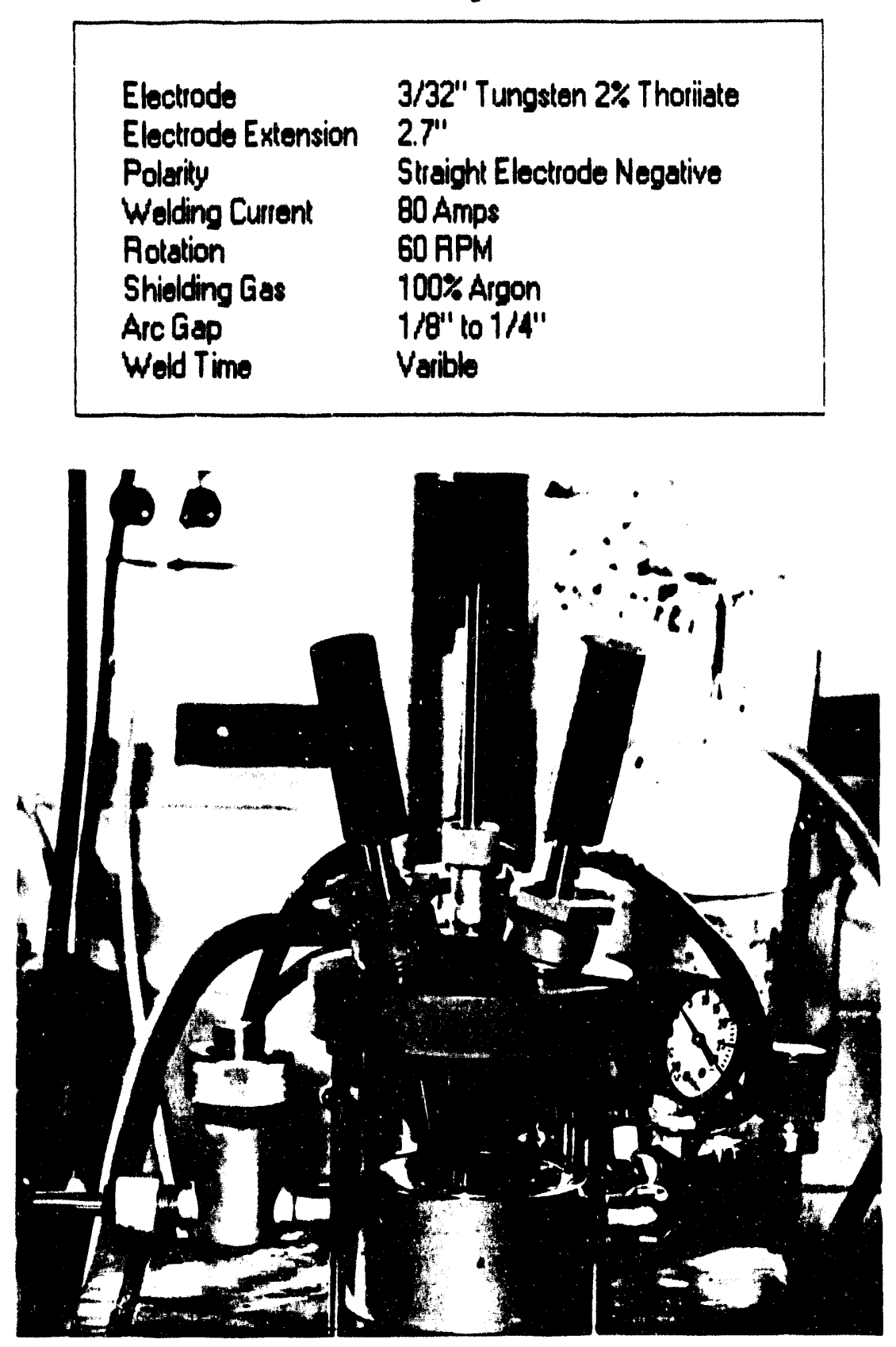

Figure 113

1

Tri-Arc Welder 


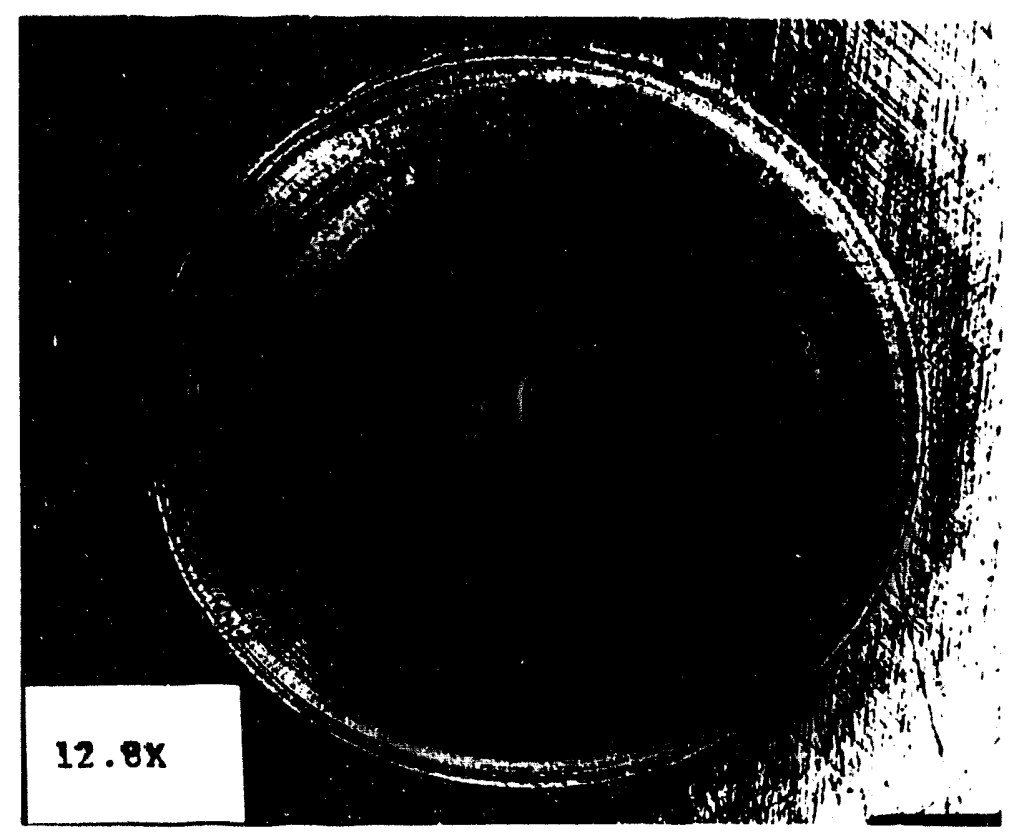

(a)

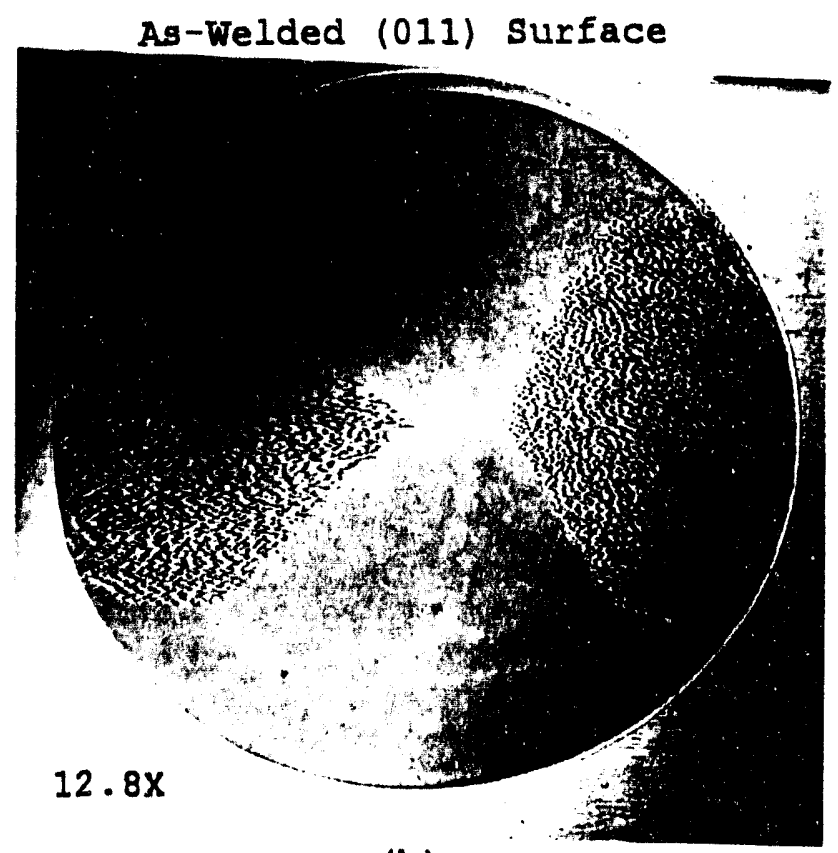

(b)

(011) Tri-Arc Weld Microstructure

Figure 114

(011) Tri-Arc Weld Various Views 


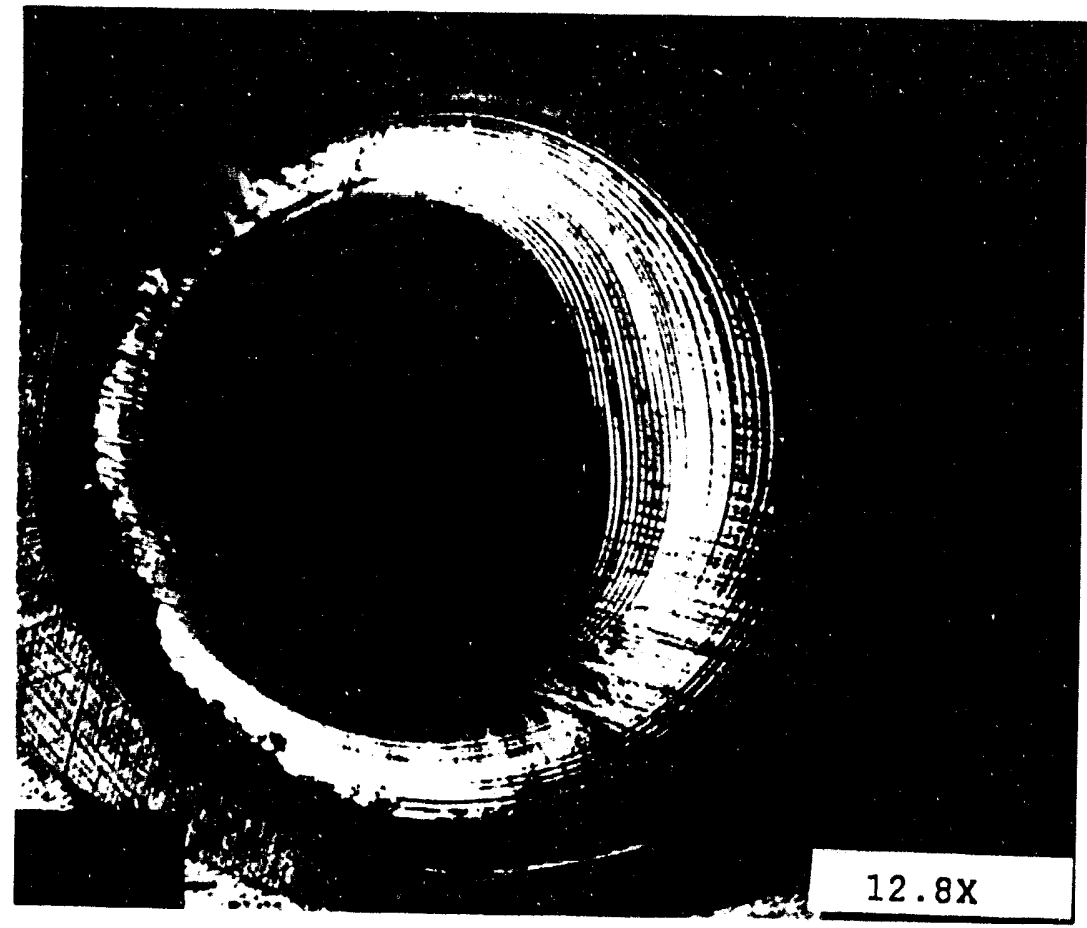

(c)

As-Welded (011) Tri-Arc Weld

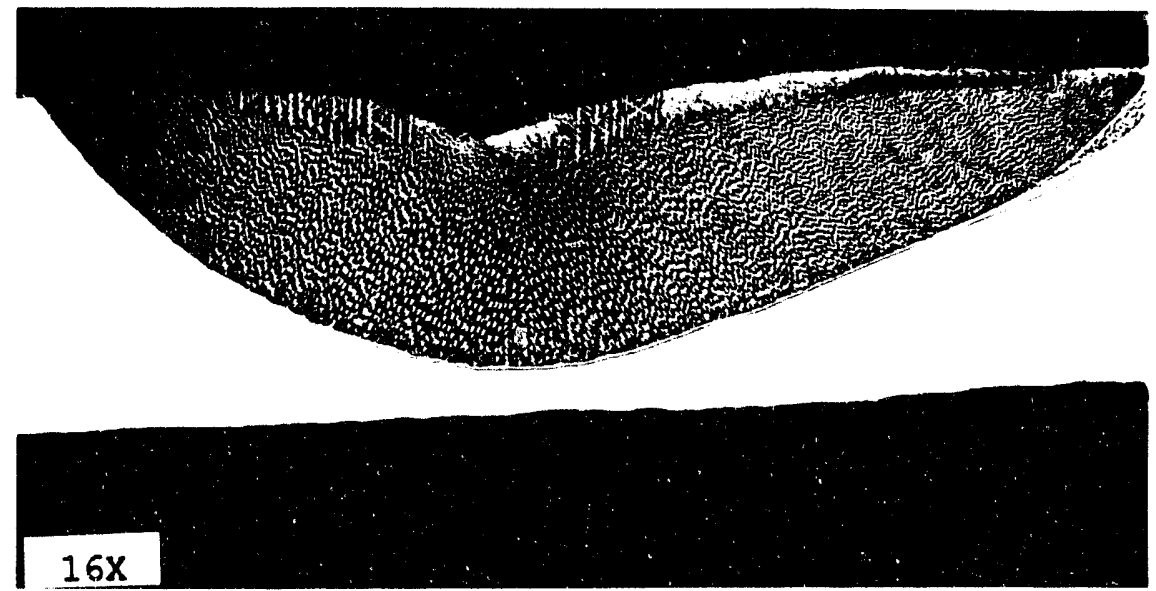

7

(d)

(011) Surface Arbitrary Cut Trangverse View

Figure 114 Continued 


\begin{abstract}
altered the penetration of the weld for a given time period.
Third, the determination of the exact center of rotation was not as predictable during welding as it was in the GTA method.
\end{abstract}




\section{Appendix D \\ Microhardness Experiments}

After welding on the single crystal, the face-centered cubic family of $\{111\} /<110\rangle$ slip lines were visible on the surface of the crystal. The active slip lines were determined by the atomic arrangement, which is the face-centered-cubic (FCC) lattice [36]. With FCC lattice the primary active slip system is given as the $\{111\}$ family of planes along the $\langle 110\rangle$ family of directions, because slip occurs along the close packed plane. For slip occurring along these specific directions, there are twelve possible slip systems which exist in the face-centeredcubic lattice [37]. These visible slip lines were the (111) family along the $\langle 110\rangle$ family of directions which appeared independent of the plane or welding method (Figure 115). To determine the extent, if any, of the hardening of the crystal by the defects caused by welding, a microhardness evaluation of the crystal was performed. The hardness measurements were taken on the $\mathrm{EB}$ welded (001) crystal along the slip lines in three crystallographic directions: [100], [110], and [010]. These measurements were taken from the edge of the weld outward. The hardness is plotted as a function of distance from the edge of the weld in Figures 116, 117, 118. Hardness values at the greatest possible distance from a weld and of an unwelded crystal 


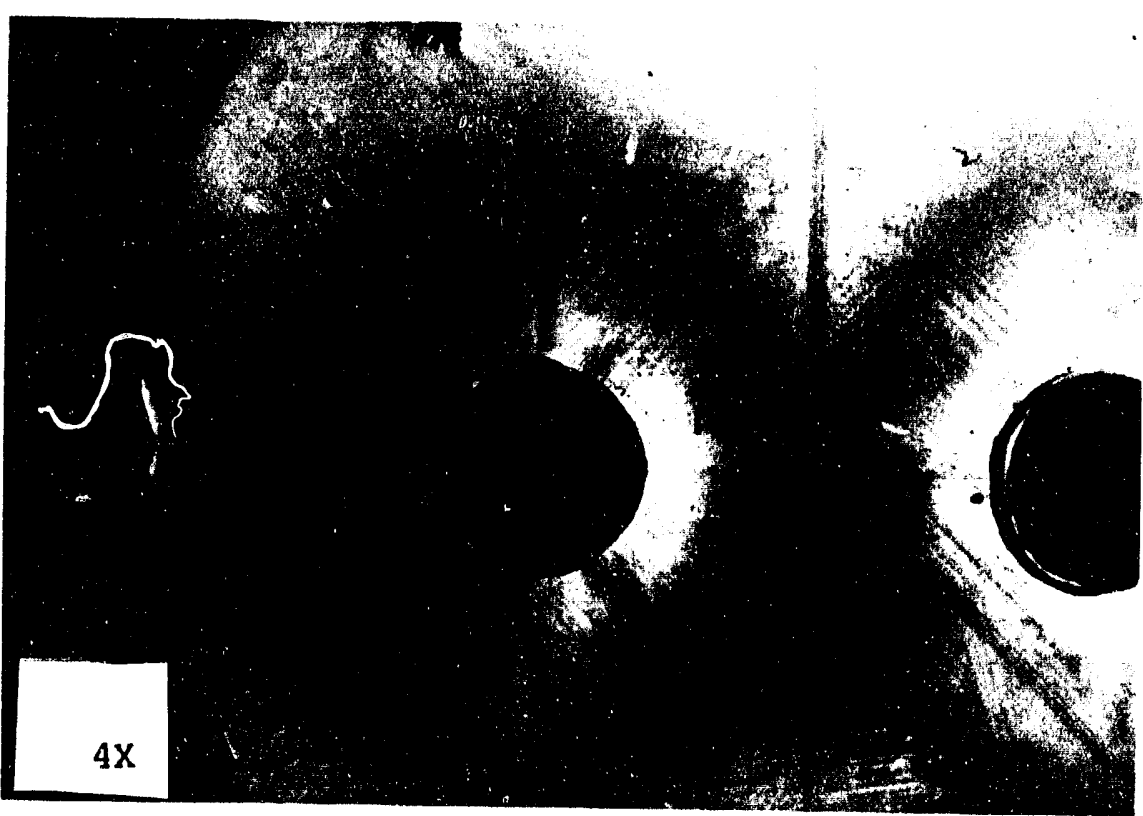

(a)

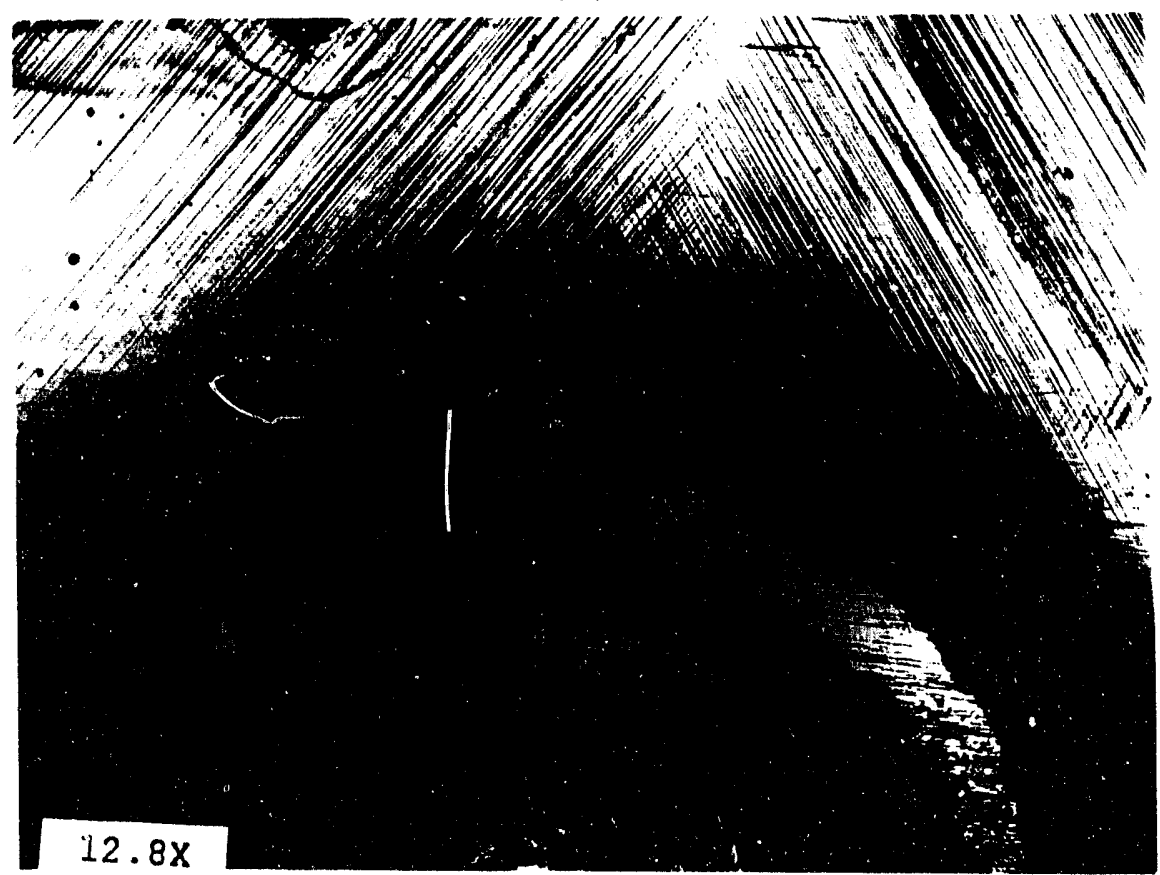

(b)

Figure 115

Slip Lines on the Welded Crystal Surface

(a) (001) EB Weld (b) (011) GTA Weld 

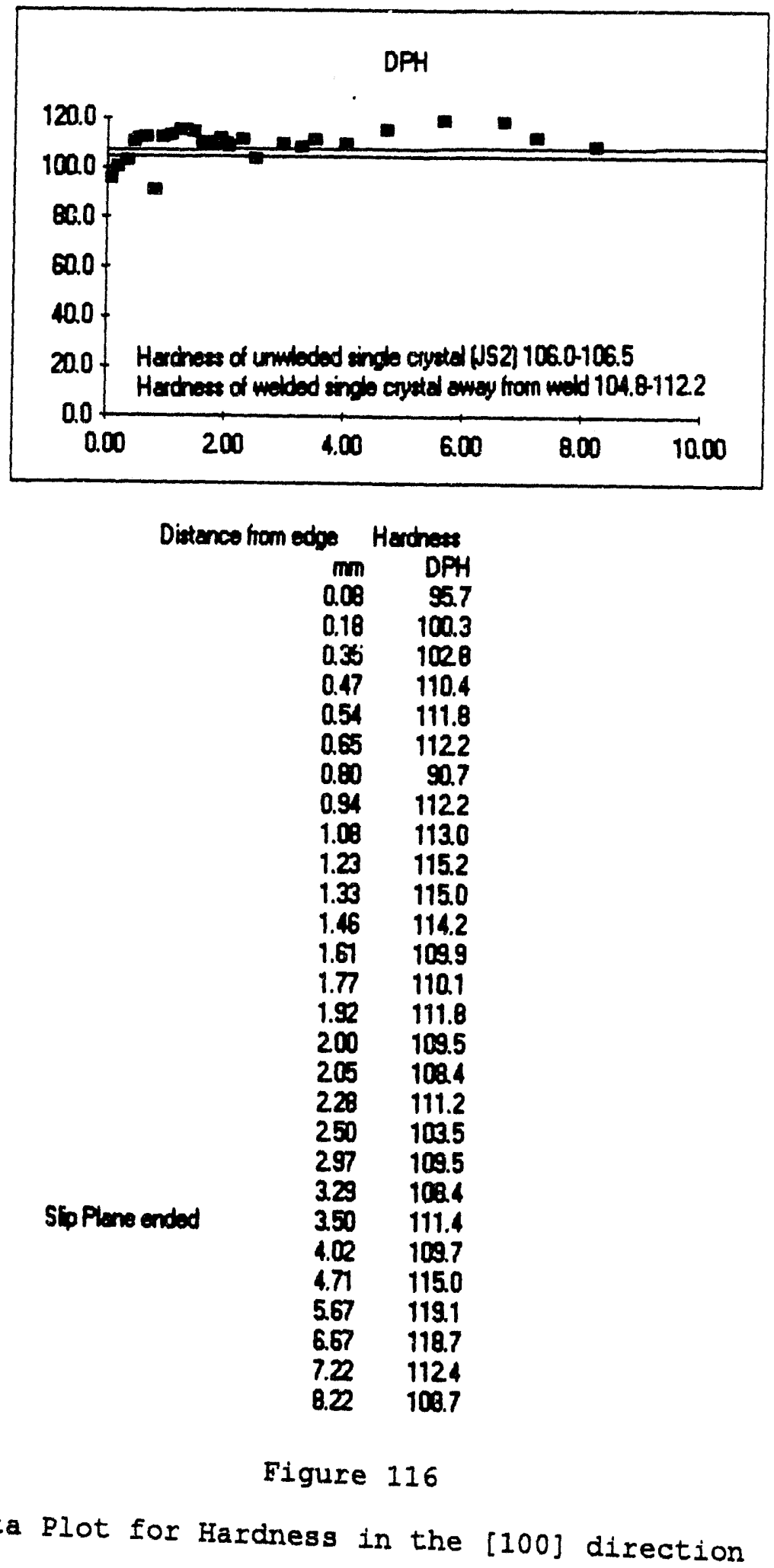


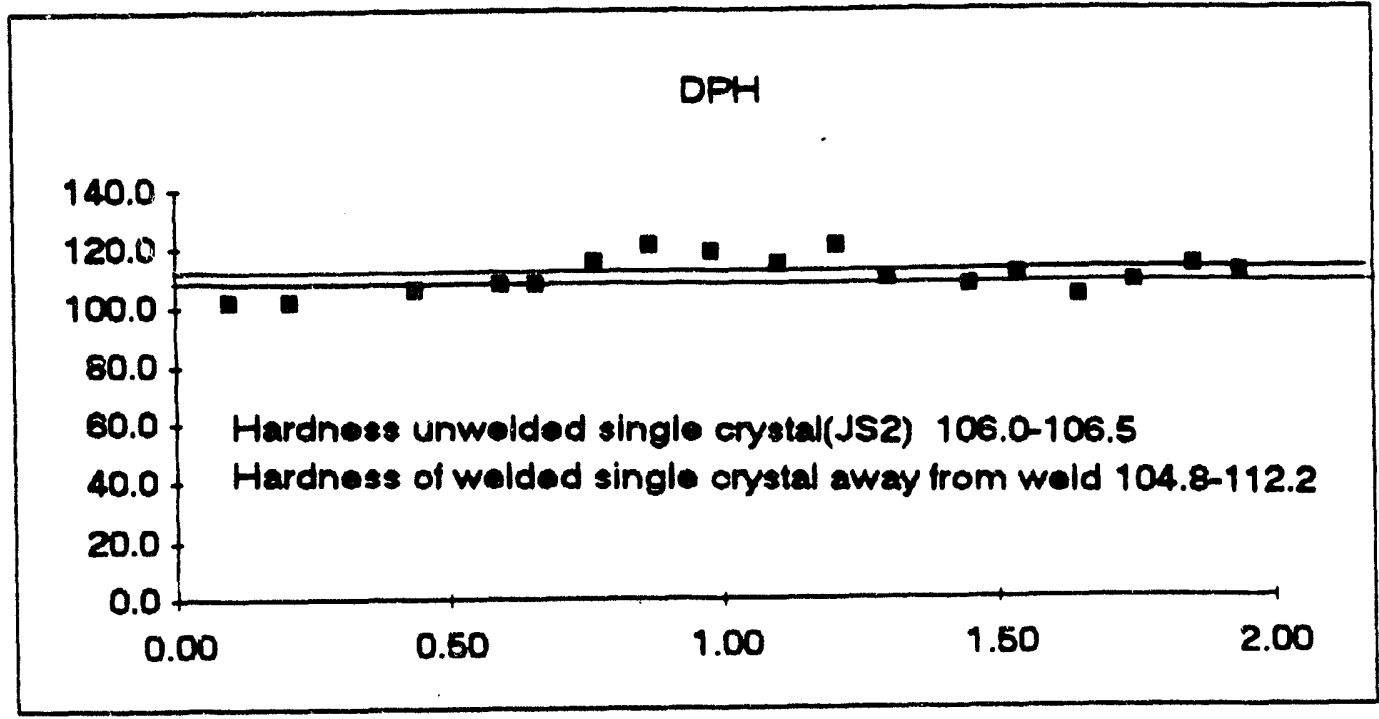

$\begin{array}{cc}\text { Distance from odge } & \text { Hardness } \\ \mathrm{mm} & \text { DPH } \\ 0.10 & 102.3 \\ 0.21 & 10 \Omega .3 \\ 0.44 & 106.0 \\ 0.60 & 107.5 \\ 0.66 & 107.5 \\ 0.77 & 115.0 \\ 0.87 & 121.0 \\ 0.98 & 118.7 \\ 1.10 & 114.2 \\ 1.21 & 120.2 \\ 1.30 & 109.9 \\ 1.45 & 106.9 \\ 1.64 & 111.0 \\ 1.65 & 103.2 \\ 1.75 & 107.8 \\ 1.86 & 113.4 \\ 1.94 & 111.2\end{array}$

Eigure 117

Data Plot for Hardness in the [010] direction 


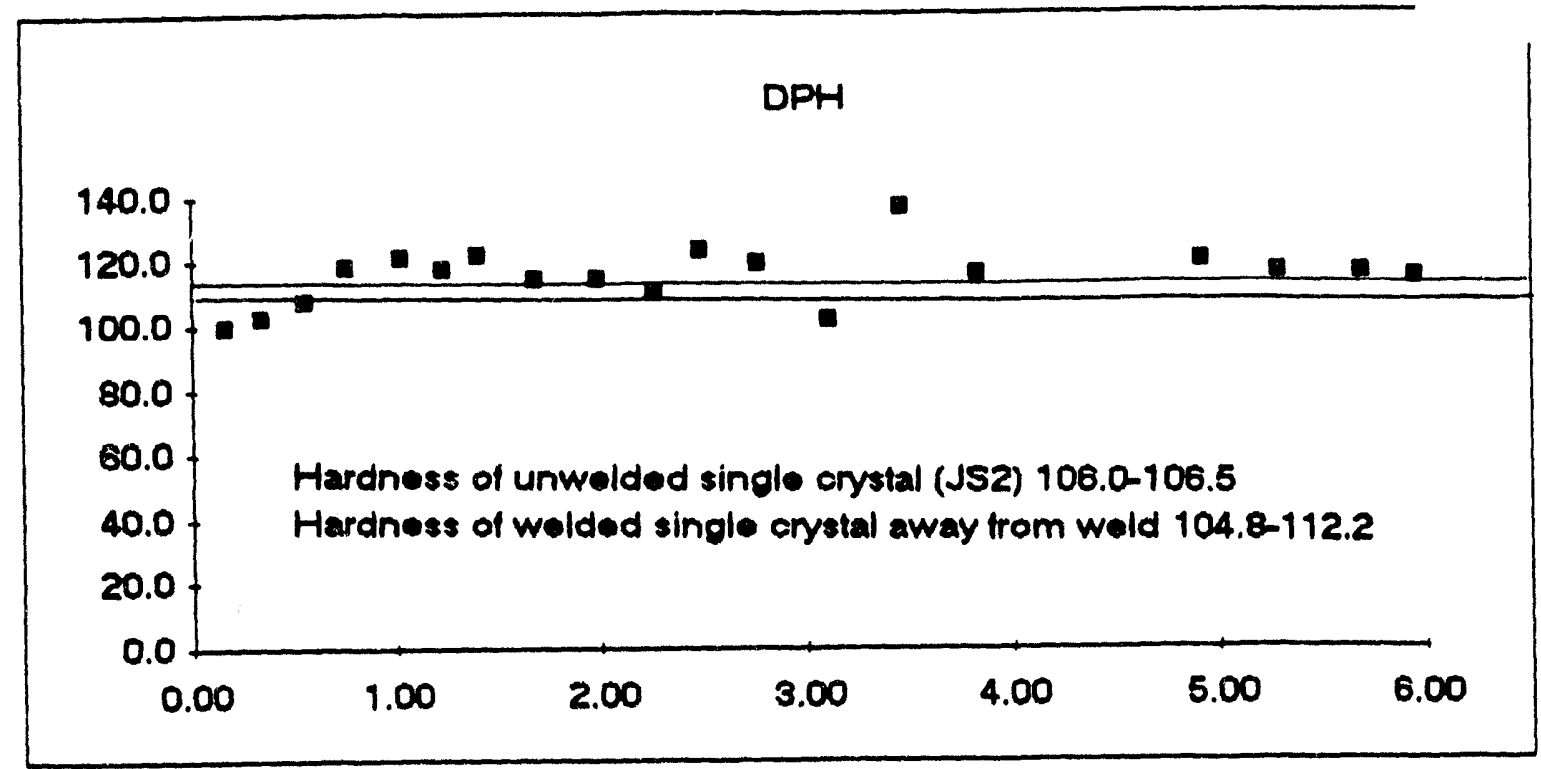

\begin{tabular}{crr}
$\begin{array}{c}\text { Distanoe from edge } \\
\mathrm{mm}\end{array}$ & \multicolumn{2}{c}{ Hardness } \\
DPH & 0.16 & 99.5 \\
0.34 & 102.8 \\
0.56 & 107.4 \\
0.76 & 118.7 \\
1.03 & 121.3 \\
1.24 & 117.8 \\
1.41 & 122.2 \\
1.68 & 115.0 \\
1.99 & 114.6 \\
2.26 & 110.3 \\
2.49 & 123.5 \\
2.76 & 119.8 \\
3.11 & 102.2 \\
3.46 & 137.2 \\
3.83 & 116.2 \\
4.92 & 120.0 \\
5.28 & 116.6 \\
5.69 & 116.2 \\
5.95 & 114.6
\end{tabular}

Figure 118

Data Plot for Hardness in the [110] direction 
were plotted for a comparative analysis to the slip line data. However, the hardness away from the weld proved to be very inconsistent, therefore an average value was plotted. The hardness values varied from less than that of an unwelded crystal to values which were comparable to those along the slip lines. The standard deviations of the hardness data ranged from 6 to 8 . The average hardiness for each of the three directions was also indicated on the appropriate figure. Since no significant trend appeared in the average hardness measured, it was determined that the hardness was independent of direction. As noted in Figure 81, the [100] direction hardness measurements taken after the slip lines had disappeared from the surface showed no appreciable variation. This indicated that there was no significant variation caused by the slip lines in the hardness over the entire welded region. 
Appendix $\mathbf{z}$

\section{Chemical Analysis Report}

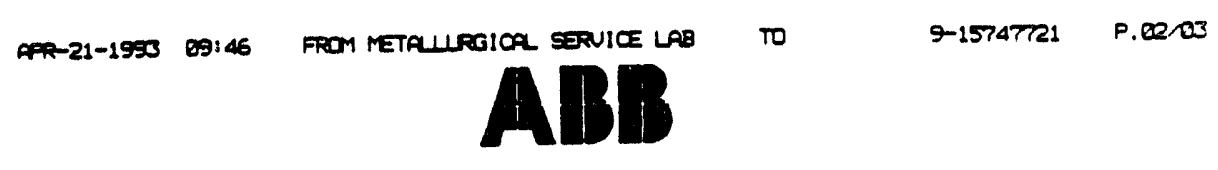

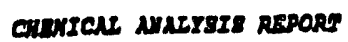

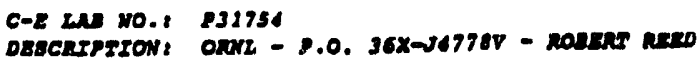

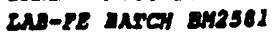

\begin{tabular}{|c|c|}
\hline $\begin{array}{l}\text { Curbon } \\
\text { Nargenese } \\
\text { Phosphorua }\end{array}$ & $\begin{array}{c}0.001 \\
<0.01 \\
0.002\end{array}$ \\
\hline $\begin{array}{l}\text { Duderz } \\
\text { asdicon } \\
\text { Nieked }\end{array}$ & $\begin{array}{c}0.001 \\
<0.02 \\
0.01\end{array}$ \\
\hline $\begin{array}{l}\text { Chrondirs } \\
\text { molybdasum } \\
\text { vanadiug }\end{array}$ & $\begin{array}{l}<0.01 \\
<0.01 \\
<0.001\end{array}$ \\
\hline $\begin{array}{l}\text { Coduablum } \\
\text { gleendue } \\
\text { cobels }\end{array}$ & $\begin{array}{r}<0.001 \\
<0.001 \\
0.003\end{array}$ \\
\hline $\begin{array}{l}\text { Copper } \\
\text { Munenen } \\
\text { Dexon }\end{array}$ & $\begin{array}{r}<0.001 \\
0.002 \\
<0.001\end{array}$ \\
\hline $\begin{array}{l}\text { Tungares } \\
\text { Areonde } \\
\text { Tin }\end{array}$ & $\begin{array}{l}<0.01 \\
<0.001 \\
0.001\end{array}$ \\
\hline 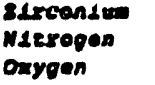 & $\begin{array}{l}60.001 \\
0.001 \\
0.021\end{array}$ \\
\hline
\end{tabular}

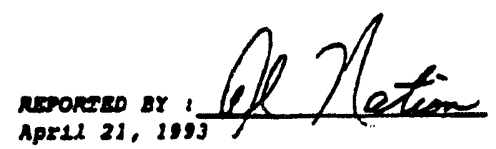

ABB C-E Power Products Manufacturing 
ORNL/TM-12623

\section{INTERNAL DISTRIBUTION}

1-10. L. A. Boatner

11-20. S. A. David

21. H. E. Harmon

22. J. B. Roberto

23-28. J. B. Sipf

29. Solid State Division Library

30. J. M. Vitek

31. C. H. Zeigler

32-33. Laboratory Records Department

34. Laboratory Records, ORNL-RC

35. Document Reference Section

36. Central Research Library

37. ORNL Patent Section

\section{EXTERNAL DISTRIBUTION}

38-47. Office of Scientific and Technical Information, P.O. Box 62, Oak Ridge, Tenn. 37831

48. Office of Assistant Manager for Energy Research and Development, Department of Energy, Oak Ridge Operations, P.O. Box 2001, Oak Ridge, Tenn. 37831

49. D. P. Corrigan, Rensselaer Polytechnic Institute, Physics Department, Troy, NY 12181

50. P. A. Curreri, Materials Scientist, National Aeronautics and Space Administration, Mail Code ES74, Space Science Laboratory, Marshall Space Flight Center, Ala. 35812

51. M. Glicksman, Rensselaer Polytechnic Institute, Materials Research Center, Troy, NY 12181

52. M. Rappaz, EPFL, Departement des Materiaux, $34 \mathrm{Ch}$. de Bellerive, $\mathrm{CH}-1007$, Lausanne, Switzerland

53. R. C. Rhome, Director, Microgravity Science and Applications Division, National Aeronautics and Space Administration Headquarters, Washington, D.C. 20546-0001

54. F. Rosenberger, Professor and Director, Center for Microgravity Research, University of Alabama, Huntsville, Research Institute Bldg. M67, Huntsville, Ala. 35899

55. F. R. Szofran, Marshall Space Flight Center, ES75, Marshall Space Flight Center, Ala. 35812 

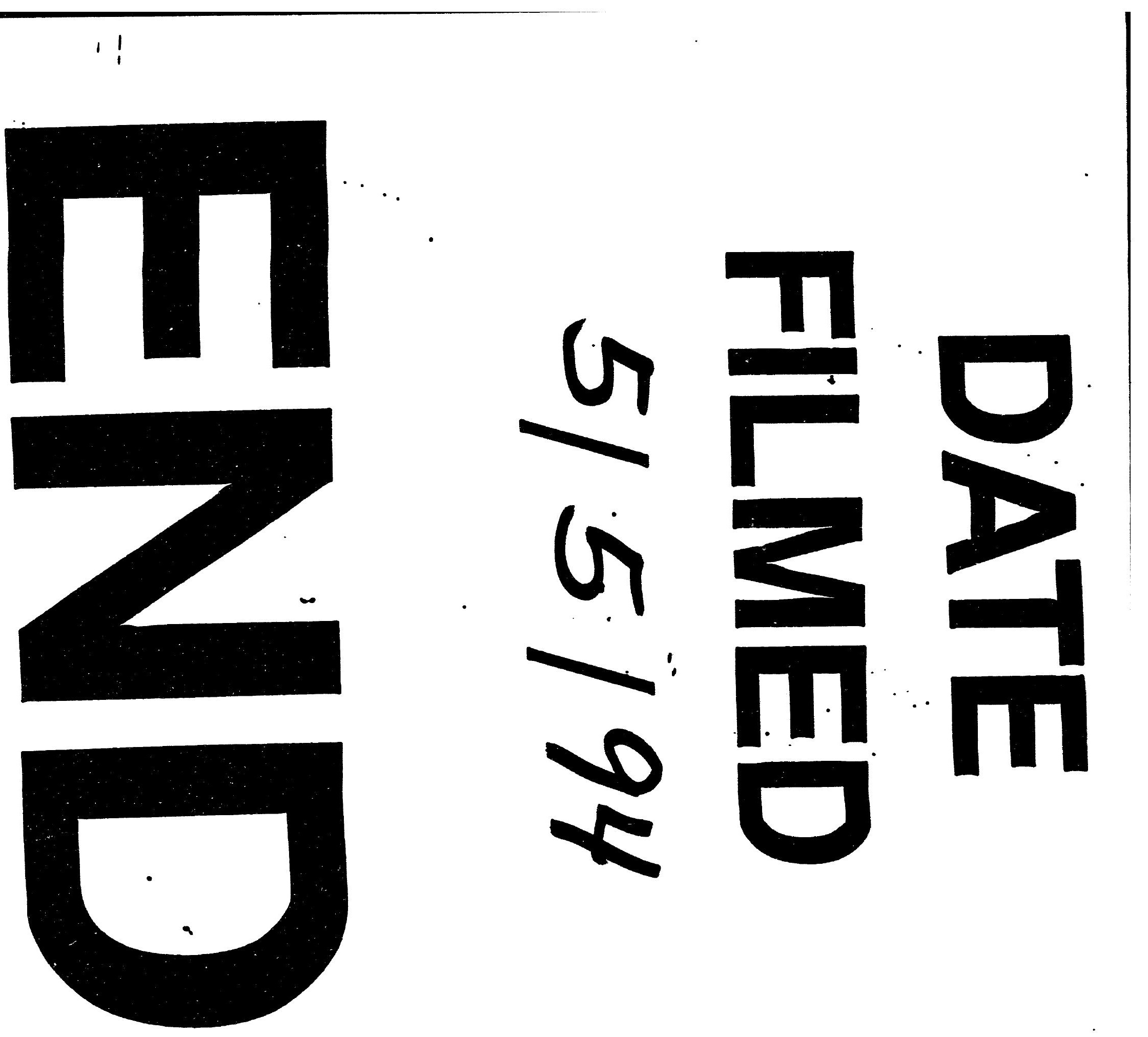
